\title{
INTERACTION AT THE SOLID-WATER INTERFACE OF OD AND 2D CARBON NANOPARTICLES: ENVIRONMENTAL IMPACTS AND APPLICATIONS FOR CONTAMINANT REMOVAL
}

\author{
A Dissertation presented to \\ the Faculty of the Graduate School at \\ the University of Missouri
}

In Partial Fulfillment

of the Requirements for the Degree

Doctor of Philosophy

by
MOHAMED BAYATI

Dr. Maria Fidalgo, Dissertation Supervisor

December 2020 
The undersigned, appointed by the Dean of the Graduate School, have examined the dissertation entitled:

\section{INTERACTION AT THE SOLID-WATER INTERFACE OF OD AND 2D \\ CARBON NANOPARTICLES: ENVIRONMENTAL IMPACTS AND APPLICATIONS FOR CONTAMINANT REMOVAL}

Presented by MOHAMED BAYATI, a candidate for the degree of Doctor of Philosophy and hereby certify that, in their opinion, it is worthy of acceptance.

\section{Maria Fidalgo}

Associate Professor of Civil and Environmental Engineering

\section{Baolin Deng}

Professor of Civil and Environmental Engineering

\section{Binbin Wang}

Assistant Professor of Civil and Environmental Engineering

\section{Jian Lin}

Associate Professor of Mechanical and Aerospace Engineering 


\section{DEDICATION}

To Enji

Thank you for always giving me the best... 


\section{ACKNOWLEDGMENTS}

First of all, I would like to thank my dissertation advisor, Dr. Maria Fidalgo, for bringing me to this interesting and promising topic and her many inspiring and valuable discussions on this research and wonderful suggestions for my graduate work. Next, I would like to thank Dr. Baolin Deng, Dr. Enos Inniss, Dr Binbin Wang, and Dr. Jian Lin for serving in my dissertation committee and their precious time and valuable comments and suggestions.

I would like to thank Dr. Zhiqiang Hu, Dr. Chung-Ho Lin, and Dr. Zheng Yan for their support during the experiments. Special thanks go to our research groups, Mohammed Numaan, Jingjing Dai, Abbas Kadhem, Zahra Salahshoor, and Sally Qasim for their help and friendship. I would also like to extend my thanks to my parents and parents in law for their support.

Finally, I would like to express my deepest gratitude to my wife, Enji Mohamed

Jamil, and my lovely daughter, Arzu Bayati for their support, encouragement, and love throughout the years. 


\section{TABLE OF CONTENTS}

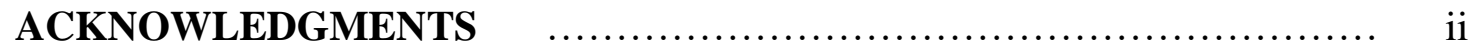

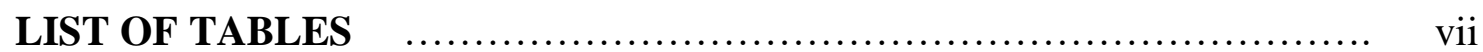

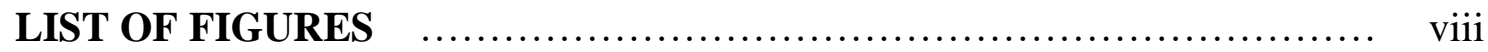

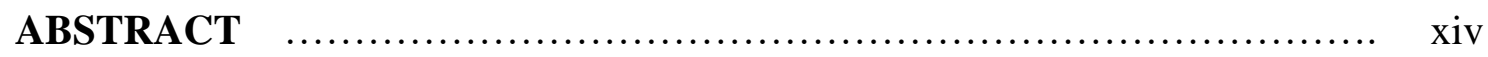

CHAPTER

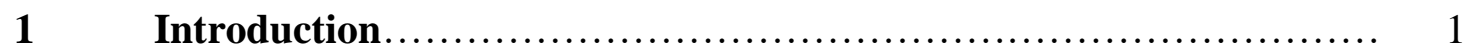

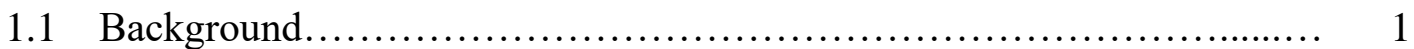

1.2 Research objectives............................................... 4

$2 \quad$ Literature Review............................................. 7

2.1 Introduction to carbon materials.................................... 7

2.2 Graphene and graphene oxide.................................... 10

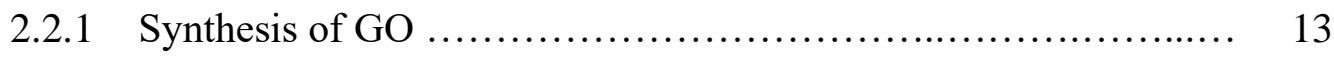

2.2.2 Graphene nanomaterials in aquatic environments............... 14

2.2.3 Aggregation behavior of GO in aquatic environments............ 15

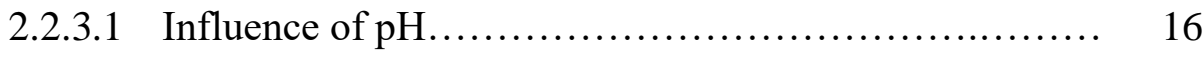

2.2.3.2 Influence of IS and salt type...................... 18

2.2.3.3 Influence of NOM................................ 20

2.3 Carbon dots.................................................... 22

2.3.1 Synthesis of CDs....................................... 24

2.3.1.1 Top-Down methods.............................. 24

2.3.1.2 Bottom-Up methods............................... 25

2.4 Laser induced graphene........................................ 26

2.4.1 LIG formation on PI under ambient conditions.................. 27

2.4.2 LIG formation on modified PI and phenolic resin............... 29 
2.4.3 LIG formation on PI under controlled atmosphere............... 30

2.4.4 Applications of LIG...................................... 31

3 Effect of Water Chemistry on The Aggregation and 35 Photoluminescence Behavior of Carbon Dots...........................

Abstract............................................................. 35

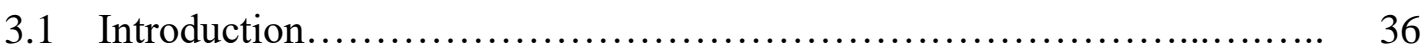

3.2 Materials and methods............................................... 40

3.2.1 Synthesis............................................. 40

3.2.2 Characterization.......................................... 41

3.2.3 DLVO Modeling.......................................... 42

3.3 Results and discussion.............................................. 44

3.3.1 Influence of ionic strength............................... 47

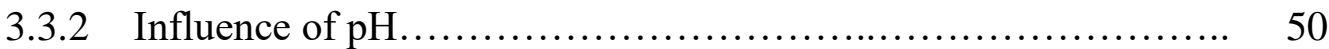

3.3.3 Influence of natural organic matter........................ 53

3.3.4 DLVO modeling........................................ 55

3.3.5 Fluorescence.............................................. 59

3.4 Conclusion.................................................... 63

4 Aggregation of graphene oxide in natural waters: the role of solution chemistry and specific interactions............................. 65

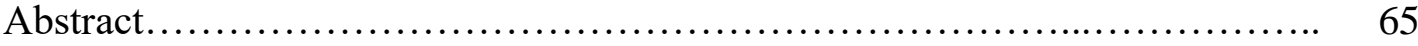

4.1 Introduction.................................................... 66

4.2 Materials and methods............................................... 69

4.2.1 Synthesis............................................... 69

4.2.2 Characterization......................................... 69

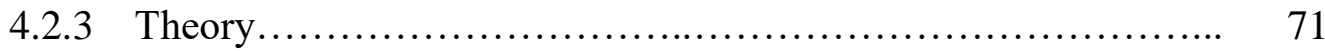

4.2.3.1 Sphere-Sphere DLVO interaction.................. 71

4.2.3.2 Plate-Plate DLVO interaction...................... 73

4.3 Results and discussion......................................... 73

4.3.1 GO Characterization..................................... 73 
4.3.2 Aggregation of GO Particles................................. 77

4.3.2.1 Impact of Salt Types and Ionic Strength................. 78

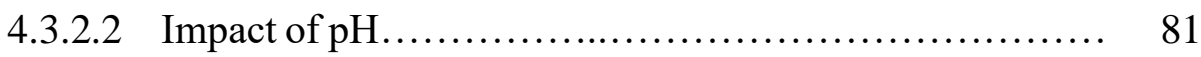

4.3.2.3 Impact of Natural Organic Matter...................... 84

4.3.3 DLVO Modeling.............................................. 88

4.4 Conclusion............................................................ 94

5 Laser Induced Graphene /Ceramic Membrane Composite: Preparation and Characterization................................ 95

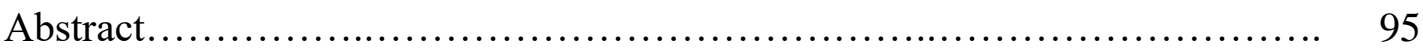

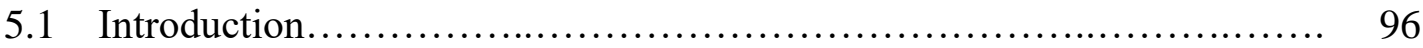

5.2 Materials and methods........................................... 98

5.2.1 Fabrication of laser induced graphene (LIG) on microfiltration ceramic membrane ........................................... 98

5.2.2 Characterization techniques................................... 100

5.2.3 Permeability and transport model.............................. 102

5.2.4 Rejection measurements.................................... 104

5.3 Results and discussion............................................ 105

5.3.1 Fabrication of asymmetric membranes........................ 105

5.3.2 Membrane Permeability.................................... 113

5.3.3 Particle Rejection.......................................... 119

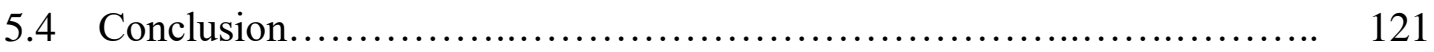

Supporting Information................................................ 123

6 Adsorption of Atrazine by Laser Induced Graphitic Material: An Efficient, Scalable and Green Alternative for Pollution Abatement..... 124

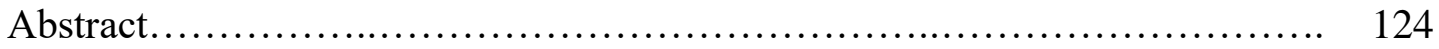

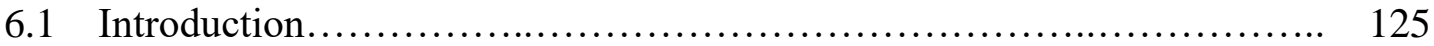

6.2 Material and methods........................................... 127

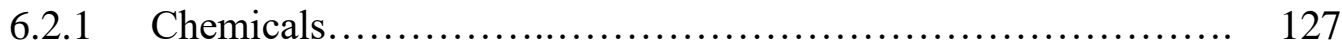

6.2.2 Preparation of laser induced graphitic material (LIG)............ 128

6.2.3 Characterization of LIG powder............................. 129 


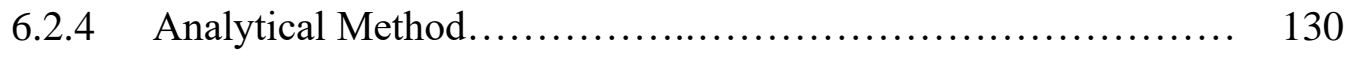

6.2.5 Adsorption experiments................................. 130

6.2.6 Data analysis........................................... 132

6.3 Results and discussions....................................... 134

6.3.1 LIG powder characterization............................. 134

6.3.2 Adsorption kinetics..................................... 141

6.3.3 Adsorption isotherms................................... 144

6.3.4 Adsorption of ATZ by LIG in the presence of HA.............. 151

6.3.5 Regeneration of LIG.................................... 152

6.4 Conclusion....................................................... 154

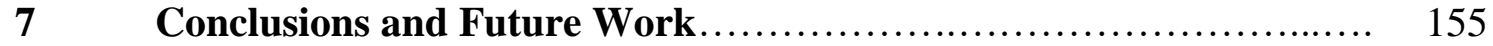

7.1 Conclusions.................................................. 155

7.2 Future work...................................................... 158

7.2.1 Investigating the performance of the prepared LIG membrane for 158

7.2.2 Investigating the removal efficiency of heavy metals from aqueous systems with thiol functionalized LIG membrane.

Bibliography .................................................... 160

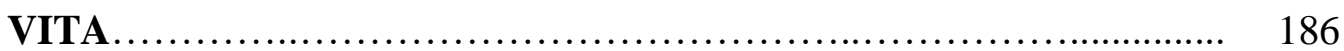




\section{LIST OF TABLES}

Table

Page

5.1 Physical properties of the solvents used for permeability test........................................................ 104

6.1 Physiochemical properties of ATZ .............................. 132

6.2 Three phase parameters of the intraparticle diffusion kinetic model....... 143

6.3 Isotherm parameters for atrazine adsorption on LIG under different $\mathrm{pH}$ and ionic strength............................................... 146

6.4 Comparison of adsorption capacity of different adsorbents for the removal of atrazine. 


\section{LIST OF FIGURES}

Figure $\quad$ Page

2.1 Overview of graphene-based nanomaterials. Graphene can be wrapped into OD fullerenes, rolled up into 1D nanotubes, or stacked into 3D graphite.......................................................................... 8

2.2 History of graphene-based nanomaterials.......................... 9

2.3 Layered structure of graphite showing the $\mathrm{sp}^{2}$ hybridized carbon atoms tightly bonded in hexagonal rings.................................. 10

2.4 HRTEM image of a graphene sheet showing the crystalline lattice. (b) A single sheet graphene can be visualized by eye...................... 11

2.5 Schematic structure of graphene oxide............................. 13

2.6 Synthesis of graphene oxide................................... 14

2.7 Configurations of the graphene-tannic acid-graphene aggregates..........

2.8 A graphic representation of top-down synthetic approaches of CDs.....

2.9 A graphic representation of bottom-up synthetic approaches of CDs. . .

2.10 LIG formation. (a) Schematic of the synthesis process of LIG from PI. (b) SEM image of LIG patterned into an owl shape at an image density of 1000 PPI. (c) SEM image of LIG film. (d) HRTEM image of LIG representing the average lattice space of $\sim 3.4 \AA$ corresponds to the $(002)$ planes of graphitic materials. (e) Cs-correction STEM image taken at the edge of a LIG flake; scale bar, $2 \mathrm{~nm}$. (f) TEM image of selected area indicated as a rectangle in (e). (g) LIGF patterned in the shape of an $\mathrm{R}$ letter; scale bar is $1 \mathrm{~mm}$. (h) LIGF with thickness of $\sim 1 \mathrm{~mm}$ made at an image density of 500 PPI; scale bar is $500 \mu \mathrm{m}$. Inset is the schematic 500 PPI. (i) SEM of LIGF (boxed in h) at higher magnification; scale bar is $2 \mu \mathrm{m}$.

2.11 In situ formation of LIG composite. (a) The preparation steps of m-PI. Energy-dispersive X-ray spectroscopy of B-LIG for (b) boron, (c) carbon, and (d) oxygen. TEM images of LIG containing new uniformed sizes of (e, g) $\quad \mathrm{Co}_{3} \mathrm{O}_{4}$ and (f, h) $\mathrm{MoO}_{2}$ crystalline nanoparticles. 
2.12 LIG formation under controlled atmosphere. (a) Scheme and (b) photograph of the apparatus for the fabrication of LIG under a controlled atmosphere. Contact angles of LIG prepared in (c) ambient air, (d) air (chamber), (e) $\mathrm{O}_{2}$ (chamber), (f) $\mathrm{Ar}$ (chamber), (g) $\mathrm{H}_{2}$ (chamber), and (h) $\mathrm{SF}_{6}$ (chamber).

2.13 LIG as membrane for oil-water separation. $(a, b)$ SEM image of LIG filter. (c) Water droplet on the surface of the LIG filter; the measured contact angle is $155^{\circ}$. (d-f) Filtration of $\mathrm{CHCl}_{3} / \mathrm{H}_{2} \mathrm{O}$ mixture with the LIG filter. (d) Water does not go through the filter; (e) $\mathrm{CHCl}_{3}$ goes through the filter; (f) water stays on top of the filter even after all of the $\mathrm{CHCl}_{3}$ went through

2.14 LIG film for microbial antifouling. (a) Biofilm growth on the PI, graphite, and LIG surfaces with P. aeruginosa showing biomass and average thickness. Representative IMARIS software images for (b) PI; (c) graphite; (d) LIG; (e) the interface between the LIG (left) and PI film (right). Green represents live bacteria, red represents dead bacteria, and blue represents EPS.

3.1 (a), (c) Representative transmission electron microscopy image of amine capped carbon dots and plain carbon dots: insets represent the segmented particles after subtracting the background using ImageJ software. (b), (d) Size distribution of a-CDs and CDs, as percent number of

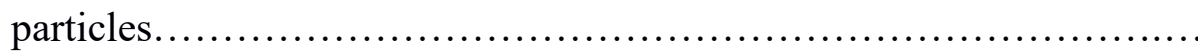

3.2 (a), (b) Representative high-resolution transmission electron microscopy images of a-CDs and CDs respectively with lattice parameters of 0.32

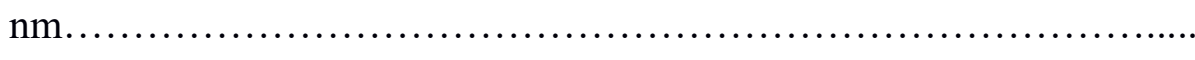

3.3 Fourier transform infrared spectroscopy spectra of: (a) a-CDs. (b) CDs.

3.4 Zeta potential of $\mathrm{CDs}(\mathrm{pH}=5.7)$ and a-CDs $(\mathrm{pH}=9.7)$ as a function of ionic strength $\left(\mathrm{NaCl}\right.$ and $\left.\mathrm{CaCl}_{2}\right)$....

3.5 Hydrodynamic diameter of $\mathrm{CDs}(\mathrm{pH}=5.7)$, a-CDs $(\mathrm{pH}=9.7)$ as a function

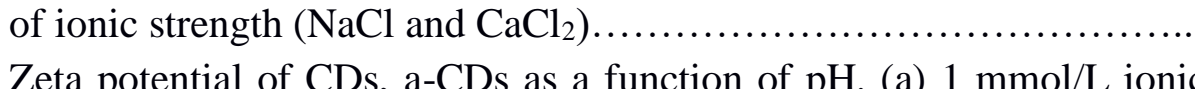

3.6 Zeta potential of $\mathrm{CDs}$, a-CDs as a function of $\mathrm{pH}$. (a) $1 \mathrm{mmol} / \mathrm{L}$ ionic strength given by $\mathrm{NaCl}$. (b) $1 \mathrm{mmol} / \mathrm{L}$ ionic strength given by $\mathrm{CaCl}_{2}$

3.7 Hydrodynamic diameter of a-CDs, CDs as a function of $\mathrm{pH}$. (a) 1 $\mathrm{mmol} / \mathrm{L}$ ionic strength given by $\mathrm{NaCl}$. (b) $1 \mathrm{mmol} / \mathrm{L}$ ionic strength given by $\mathrm{CaCl}_{2}$ 
3.8 Hydrodynamic diameter (a) and zeta potential (b) of CDs, a-CDs suspended in humic acid solutions of variable concentration at neutral

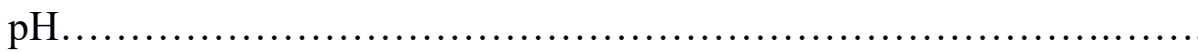

3.9 Calculated DLVO interaction energy plotted as a function of separation distance between CDs particles; effect of ionic strength and $\mathrm{pH}$ variations. (a) CDs with different $\mathrm{NaCl}$ concentrations. (b) $\mathrm{CDs}$ with different $\mathrm{CaCl}_{2}$ concentrations. (c) $\mathrm{CDs}$ in $1 \mathrm{mmol} / \mathrm{L} \mathrm{NaCl}$ with different $\mathrm{pH}$. (d) $\mathrm{CDs}$ in $1 \mathrm{mmol} / \mathrm{L} \mathrm{CaCl}_{2}$ with different $\mathrm{pH}$.

3.10 Calculated DLVO interaction energy plotted as a function of separation distance between a-CDs particles; effect of ionic strength and $\mathrm{pH}$ variations. (a) a-CDs with different $\mathrm{NaCl}$ concentrations. (b) a-CDs with different $\mathrm{CaCl}_{2}$ concentrations. (c) a-CDs in $1 \mathrm{mmol} / \mathrm{L} \mathrm{NaCl}$ with different $\mathrm{pH}$. (d) a-CDs in $1 \mathrm{mmol} / \mathrm{L} \mathrm{CaCl} 2$ with different $\mathrm{pH}$..............

3.11 Fluorescence spectra for (a) CDs, and (b) a-CDs, at different concentrations.

3.12 Fluorescence spectra for (a) CDs, and (b) a-CDs, at various levels of ionic strength and electrolytes.

3.13 Absorption and fluorescence spectra: CDs (a) and (b); a-CDs(c) and (d), respectively, in the presence of $\mathrm{HA}$; comparison of fluorescence intensity peak for CDs, a-CDs suspended in HA solutions $(1 \mathrm{mg} / \mathrm{L}$ $100 \mathrm{mg} / \mathrm{L}$ ) and pure HA (e)

4.1 Representative transmission electron microscopy (TEM) image of GO (a) 2D structure in absence of ionic strength, (b) 3D aggregated structure, (c) folded, aggregated structure. (d) Atomic Force Microscopy (AFM) of GO.

4.2 (a) Fourier-Transform Infrared Spectroscopy (FTIR) of graphite and GO. (b) X-ray diffraction patterns of GO.

4.3 UV-Vis absorption spectra and standard curve (insert) of graphene oxide

4.4 XPS spectra of GO. (a) Survey XPS and (b) C 1s XPS spectra

4.5 Zeta potential of $\mathrm{GO}$ as a function of ionic strength $\left(\mathrm{NaCl}\right.$ and $\left.\mathrm{CaCl}_{2}\right)$ at $\mathrm{pH} 5.2$.

4.6 Hydrodynamic diameter of $\mathrm{GO}$ as a function of ionic strength $(\mathrm{NaCl}$ and $\mathrm{CaCl}_{2}$ ) at $\mathrm{pH} 5.2$.

4.7 Zeta potential of $\mathrm{GO}$ as a function of $\mathrm{pH}$ : (a) $\mathrm{GO}$ in $10 \mathrm{mM} \mathrm{NaCl}$; (b) $\mathrm{GO}$ in $0.5 \mathrm{mM} \mathrm{CaCl}_{2}$

4.8 Hydrodynamic diameter of GO as a function of $\mathrm{pH}$ (a) $\mathrm{GO}$ in10 mM $\mathrm{NaCl}$. (b) $\mathrm{GO}$ in $0.5 \mathrm{mM} \mathrm{CaCl}_{2}$. 
4.9 Zeta potential of $\mathrm{GO}$ as a function of $\mathrm{pH}$. (a) in presence of $5 \mathrm{ppm}, 10$ ppm $\mathrm{HA}$ and TA respectively with $1 \mathrm{mM}$ of $\mathrm{NaCl}$. (b) in presence of 5 ppm, $10 \mathrm{ppm} \mathrm{HA}$ and TA respectively with $1 \mathrm{mM} \mathrm{CaCl}_{2} \ldots \ldots \ldots \ldots \ldots . . . . .$.

4.10 Hydrodynamic diameter of $\mathrm{GO}$ as a function of $\mathrm{pH}$. (a) in presence of 5 ppm, 10 ppm HA and TA respectively with $1 \mathrm{mM}$ of $\mathrm{NaCl}$. (b) in presence of $5 \mathrm{ppm}, 10 \mathrm{ppm} \mathrm{HA}$ and TA respectively with $1 \mathrm{mM} \mathrm{CaCl}_{2} \ldots$

4.11 Sphere-sphere DLVO modeling; effect of ionic strength and $\mathrm{pH}$ variations. (a) $\mathrm{GO}$ with different $\mathrm{NaCl}$ concentrations. (b) $\mathrm{GO}$ with different $\mathrm{CaCl}_{2}$ concentrations. (c) $\mathrm{GO}$ in $10 \mathrm{mM} \mathrm{NaCl}$ with different

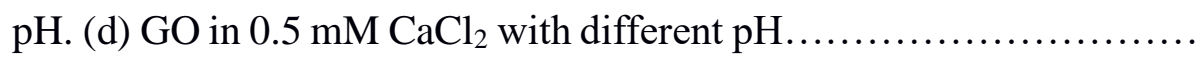

4.12 Sphere-sphere DLVO modeling; effect of humic acid and $\mathrm{pH}$ variations. (a) $\mathrm{GO}$ in $1 \mathrm{mM} \mathrm{NaCl}$ with $5 \mathrm{mg} / \mathrm{L} \mathrm{HA}$. (b) $\mathrm{GO}$ in $1 \mathrm{mM} \mathrm{NaCl}$ with 10 $\mathrm{mg} / \mathrm{L} \mathrm{HA}$ (c) GO in $1 \mathrm{mM} \mathrm{CaCl}_{2}$ with $5 \mathrm{mg} / \mathrm{L}$ HA. (d) $\mathrm{GO}$ in $1 \mathrm{mM} \mathrm{CaCl}_{2}$ with $10 \mathrm{mg} / \mathrm{L} \mathrm{HA}$

4.13 Sphere-sphere DLVO modeling; effect of tannic acid and $\mathrm{pH}$ variations. (a) $\mathrm{GO}$ in $1 \mathrm{mM} \mathrm{NaCl}$ with $5 \mathrm{mg} / \mathrm{L} \mathrm{TA}$. (b) $\mathrm{GO}$ in $1 \mathrm{mM} \mathrm{NaCl}$ with 10 $\mathrm{mg} / \mathrm{L} \mathrm{TA}$ (c) GO in $1 \mathrm{mM} \mathrm{CaCl}_{2}$ with $5 \mathrm{mg} / \mathrm{L} \mathrm{TA}$. (d) $\mathrm{GO}$ in $1 \mathrm{mM} \mathrm{CaCl}_{2}$ with $10 \mathrm{mg} / \mathrm{L} \mathrm{TA}$.

4.14 Plate-plate DLVO modeling; effect of ionic strength and $\mathrm{pH}$ variations. (a) $\mathrm{GO}$ with different $\mathrm{NaCl}$ concentrations. (b) $\mathrm{GO}$ with different $\mathrm{CaCl}_{2}$ concentrations. (c) $\mathrm{GO}$ in $10 \mathrm{mM} \mathrm{NaCl}$ with different pH. (d) $\mathrm{GO}$ in 0.5 $\mathrm{mM} \mathrm{CaCl}_{2}$ with different $\mathrm{pH}$.

5.1 General procedure for the preparation of laser induced graphene/ceramic membrane (LIG/CM) composite.

5.2 Digital images of (a) Ceramic membrane before coating. (b) Ceramic membrane after coating with PI. (c) Ceramic membrane after laser treatment of PI.

5.3 Permeability experimental set up.

5.4 (a) FTIR spectroscopy of Kapton film and PI sheet. (b) XRD patterns of laser treated Kapton film.

5.5 (a) Raman spectra of LIG on the ceramic membrane. (b) XPS spectra of LIG.

5.6 BET surface area measurement of LIG. (a) $\mathrm{N}_{2}$ adsorption isotherm. (b) pore size distribution.

5.7 Water contact angles (WCA) with a $5 \mu \mathrm{L}$ water droplet of LIG on ceramic membrane

5.8 3D optical profilometry images of (a) a membrane surface with no PI or LIG coating. (b) a membrane surface with PI coating. (c) a membrane 
surface with LIG coating. (d) Data comparison of roughness values collected using optical profilometry of uncoated membrane surfaces, PI coated membrane surfaces, and LIG coated membrane surfaces.

5.9 Scanning electron microscopy (SEM) of the ceramic support surface (a) before coating. (b) after coating with PI. (c) after one layer of PI was converted to LIG. (d) after two layers of PI were converted to LIG. (e) after four layers of PI were converted to LIG....

5.10 Scanning electron microscopy (SEM) of cross-section area (a) $\mathrm{PI} /$ ceramic membrane composite with PI thickness of $15 \pm 2 \mu \mathrm{m}$. (b) LIG/ceramic membrane composite with LIG thickness of $40 \pm 3 \mu \mathrm{m} . . .$. .

5.11 HRTEM image of LIG; scale bar, $5 \mathrm{~nm}$. Average lattice space of $\sim 3.4$ $\AA$, that corresponds to the (002) planes of graphitic materials

5.12 Pure solvent flux versus TMP for ceramic membrane coated with LIG at $20^{\circ} \mathrm{C}$

5.13 Permeate fluxes as a function of (a) dielectric constant. (b) dipole moment.

5.14 Fluxes versus TMP/ $\mu$ for $\mathrm{LIG}$ membrane at $20^{\circ} \mathrm{C}$.

5.15 Flux and viscosity $\mathrm{J}_{\mathrm{x}} \times \mu_{\mathrm{x}}$ as a function of $\mathrm{H}$-bonding contribution of Hansen solubility parameter $\delta_{h}$. Where x stands for solvent x............

5.16 Particle rejection of uncoated ceramic membrane (UNCM) and LIG/CM vs. average particle size; test solutions were composed of silica particles suspended in methanol.

5.17 Schematic of the cross-section of membranes with effective layers composed of (a) Laser induced graphene (LIG); and (b) horizontally compacted graphene oxide (GO) sheets...

5.1s Calibration curves using DLS method for (a) SP with size of 230-265 nm. (b) ST-ZL with size of 70-100 nm. (c) ST-30-LH with size of 40-50 $\mathrm{nm}$. (d) ST-O with size of 10-20 nm

6.1 General fabrication procedure of laser induced graphitic material (LIG).

6.2 $\mathrm{N}_{2}$ adsorption isotherm and pore size distribution (insert) of laser induced graphitic material (LIG).

6.3 Zeta potential of laser induced graphitic material (LIG) as a function of $\mathrm{pH}$.

6.4 Raman spectrum of laser induced graphitic material (LIG) film obtained from $10 \mathrm{~W}$ laser power and $15 \mathrm{~cm} / \mathrm{s}$ raster speed

6.5 XRD patterns of powdered laser induced graphitic material (LIG) 
6.6 FTIR spectroscopy of polyimide (PI) and laser induced graphitic material (LIG).

6.7 Scanning electron microscopy (SEM) of (a) the surface of PI and LIG. (b) cross-section view of PI/LIG composite with LIG thickness of 107.4 $\pm 3.8 \mu \mathrm{m}$.

6.8 Transmission electron microscopy (TEM) images of LIG. (a) TEM image of LIG flakes. (b) High-resolution TEM (HRTEM) of LIG shows the average lattice space of $3.4 \AA$ corresponds to the (002) planes of graphitic materials.

6.9 XPS spectra. (a) A wide scan XPS spectra of LIG. (b) High-resolution XPS of C1s peaks. (c) High-resolution XPS of N1s peak. (d) Highresolution XPS of O1s peak..............................................

6.10 Kinetics of atrazine (ATZ) adsorption on laser induced graphitic material $(\mathrm{LIG})(\mathrm{pH}=6.0, \mathrm{ATZ}$ concentration $=5 \mathrm{mg} / \mathrm{L}$, volume $=100 \mathrm{ml}$, LIG dosage $=40 \mathrm{mg}$, room temperature)

6.11 Linear regressions of kinetics plot obtained for atrazine (ATZ) removal by laser induced graphitic material $(\mathrm{LIG})(\mathrm{pH}=6.0$, ATZ concentration $=$ $5 \mathrm{mg} / \mathrm{L}$, volume $=100 \mathrm{ml}$, LIG dosage $=40 \mathrm{mg}$ and room temperature): (a) Pseudo-first order model, (b) Pseudo-second order model, (c) Intraparticle diffusion model.

6.12 Adsorption isotherms of atrazine (ATZ) on laser induced graphitic material (LIG) at different $\mathrm{pH}$ fitted with Langmuir model $(\mathrm{pH}=3,6,9$, ATZ concentration $=1-10 \mathrm{mg} / \mathrm{L}$, volume $=50 \mathrm{ml}, \mathrm{LIG}$ dosage $=10 \mathrm{mg}$ and room temperature)

6.13 (a) Adsorption isotherms of atrazine (ATZ) on laser induced graphitic material (LIG) at different ionic strength fitted with Langmuir model (IS $=1,10,100 \mathrm{mM}, \mathrm{pH}=6, \mathrm{ATZ}$ concentration $=1-10 \mathrm{mg} / \mathrm{L}$, volume $=50$ $\mathrm{ml}, \mathrm{LIG}$ dosage $=10 \mathrm{mg}$ and room temperature). (b) Possible interactions of ATZ with LIG.

6.14 Adsorption isotherms of atrazine (ATZ) by laser induced graphitic material (LIG) in the presence of humic acid (HA) added at different stages. HA concentration in pre- and post-HA introduction was $5 \mathrm{mg} / \mathrm{L}$. All experiments preformed at $\mathrm{pH}=6.0$, ionic strength of $10 \mathrm{mM}$ and room temperature

6.15 Adsorption of ATZ by LIG in consecutive cycles of use and regeneration (ATZ concentration $=5 \mathrm{mg} / \mathrm{L}, \mathrm{LIG}$ dose $=10 \mathrm{mg}$, agitating for $24 \mathrm{~h}$, room temperature) 


\begin{abstract}
Carbon dots (CDs) and graphene oxide (GO) are rapidly emerging carbon-based nanomaterials that, due to their growing applications, will inevitably find their way to natural waters; however, their environmental fate is mostly unknown. Carbon dots with different surface functionality and graphene oxide were fabricated and characterized. Their surface charge, given by the zeta potential, and their hydrodynamic diameter in suspension were investigated under a variety of environmentally relevant conditions. The effect of ionic strength was studied in the presence of monovalent $(\mathrm{NaCl})$ and divalent $\left(\mathrm{CaCl}_{2}\right)$ cations, for $\mathrm{pH}$ levels from 3 to 11 ; humic acid was used as a model for dissolved natural organic matter. Total potential energies of interactions were modeled by classical DLVO theory. The experimental results for CDs showed that water chemistry altered the surface charge of the nanomaterials, but their hydrodynamic size could not be correlated to those changes. Nanoparticles remained largely stable in suspension, with some exception at the highest ionic strength considered. DLVO theory did not adequately capture the aggregation behavior of the system. Moreover, cation and/or humic acid adsorption negatively affected the emission intensity of the particles, suggesting limitations to their use in natural water sensing applications.

The results for GO showed that the GO is negatively charged over a wide range of $\mathrm{pH}$ and the $\mathrm{pH}$ did significantly affect GO stability at a level of 4 or higher, but the particles became unstable below $\mathrm{pH} 3$ due to protonation of $-\mathrm{COOH}$ at the edge. Ionic strength (IS) and salt type had observable effects on stability as a result of electrical double layer compression and specific interactions. $\mathrm{CaCl}_{2}$ affects $\mathrm{GO}$ more noticeably than $\mathrm{NaCl}$ because of the binding ability of $\mathrm{Ca}^{2+}$ ions with carboxyl and hydroxyl functional groups.


The applicability of DLVO theory as a predictive tool was investigated by modeling GO sheets in two different geometries; three-dimensional sphere like particles and twodimensional particles at different values of $\mathrm{pH}$ and IS. Overall, the specific interactions and chemical structure of adsorbed organics had a dominant role in GO stability.

Laser induced graphene (LIG) can be fabricated in one-step, scalable, reagent-free process, by irradiation of a commercial polyimide (PI film) by a $\mathrm{CO}_{2}$ infrared laser under ambient conditions. This approach is environmentally and economically promising fabrication method. Laser induced graphene (LIG) was successfully fabricated on microporous ceramic membranes. The surface area, morphology, and chemical characterizations were performed on the LIG layer. Water contact angle measurements showed the hydrophobicity of LIG. Pure water and solvents with different polarities were used to understand the solvent flux behavior of LIG membrane. The LIG membrane showed very high non-polar solvent fluxes and remarkably low water permeability, and thus, the transport through the LIG membrane is related to dipole moment and dielectric constant, represented by solvent polarity. The LIG membrane achieved $90 \%$ rejection for $255 \mathrm{~nm}$ diameter silica particles, suggesting the presence of submicron size connecting pore channels that dominate the transport mechanism. Furthermore, LIG was used as adsorbent for the removal of atrazine (ATZ) from water. The effect of water chemistry, presence of humic acid (HA), adsorption time, and initial ATZ concentration on the adsorption process was explored. The prepared LIG exhibited significant removal of ATZ from aqueous solutions; hydrophobicity and $\pi-\pi$ interactions played important roles in the process. Adsorption of ATZ on LIG followed a pseudo-second order kinetic model and Langmuir model for the isotherm with maximum adsorption capacity of $15.0 \mathrm{mg} \mathrm{ATZ/g}$ 
LIG. Adsorption was more favorable at higher $\mathrm{pH}$ and not affected by ionic strength. LIG exhibited enhanced performance over many previously reported adsorbents. The introduction stage of HA with respect to ATZ influenced the results: pre-introduction of HA reduced ATZ adsorption by 32\%, whereas post-introduction of HA resulted in a slight release of ATZ from the LIG. 


\section{CHAPTER ONE \\ Introduction}

\subsection{Background}

Interest for both fundamental research and applications has surged dramatically for graphene-based nanomaterials and carbon nanodots (CDs) [1-3]. Thus, it is inevitable that such materials will find their way into natural water sources where, unlike the more well-studied fullerenes and carbon nanotubes, their environmental impact and fate is mostly unknown. CDs constitute a fascinating class of recently discovered nanocarbons that comprise discrete, quasi-spherical nanoparticles with sizes below $10 \mathrm{~nm}$ [1]. Because CDs display size and excitation wavelength-dependent photoluminescence behavior, can be derived from inexpensive and abundant materials, and have presumably lower environmental impact, they are attractive alternatives to metal-based quantum dots (such as $\mathrm{CdS}$ and CdTe) especially for bio applications and aqueous-phase sensing. Early studies have shown C-dots are biocompatible[4] and less cytotoxic than Cd-based quantum dots [5] and may even inhibit cancer cells [6]. Similar to C-dots, graphene and

graphene-based materials, 2D or quasi 2D materials composed of layers of $\mathrm{sp}^{2}$-hybridized carbon atoms joined by covalent bonds, display exceptional properties (mechanical, electrical, chemical) consequently increasing demand significantly. The increase in activity for these emerging CNMs, CDs and graphene-based materials, make their appearance in natural waters unavoidable-yet they are virtually unstudied. While some studies have been conducted on in-vivo toxicity and cytotoxicity of graphene-based 
materials with mixed results $[7,8]$, few studies of their aquatic fate are known $[9,10]$. Pristine graphene (or single layer graphene) is highly hydrophobic and therefore cannot be dispersed in water without the aid of a surfactant $[11,12]$. However, many surfaceactive compounds can be found in domestic and industrial wastewaters, and therefore the potential for graphene dispersion in natural waters cannot be completely discounted. Furthermore, natural organic matter (e.g. humic acid) and exopolymeric substances (e.g. proteins and polysaccharides) have been shown to adsorb onto carbon nanotubes and act as stabilization agents preventing their agglomeration and sedimentation, thus enhancing their dispersion [13]. The similarities in the structure of carbon nanotubes and graphene suggest the possibility of similar physicochemical behavior, although there are no experimental studies to confirm it. Graphene oxide (GO), on the other hand, has numerous surface groups such as hydroxyl, carboxyl, and epoxy groups that render it mostly hydrophilic. GO is negatively charged over all environmental pHs (4-10) [9,14], and thus electrostatic interaction prevents individual sheets form aggregating. Increased ionic strength induces aggregation through compression of the electrical double layer [15], but this effect is dependent on size and $\mathrm{C} / \mathrm{O}$ ratios, which in turn determine the relative importance of van der Waals attraction to electrostatic repulsion forces in stability [16]. Humic acid was observed to suppress, at least partially, this destabilization effect [9]. Reduced graphene oxide (rGO) has a similar structure to graphene, with some remaining hydroxyl and carboxylic groups on the edges of the $\mathrm{sp}^{2}$ - hybridized carbon layers due to incomplete reduction. Due to the existence of both oxidant and reductant conditions in natural waters, the potential for interconversion of the three forms of graphene is evident. UV irradiation in laboratory conditions has also resulted in fast 
transformation in the presence of $\mathrm{TiO}_{2}$ catalysts, and at slower rates in the absence of catalysts. This hints to the possible photodegradation of graphene materials in the environment. Due to their large surface area and activity, adsorption is expected to play an important role in the fate and transport of CNMs [9]. In the particular case of graphene, adsorption may alter the surface characteristics of the nanoparticles so that they become dispersed and thus mobile in aqueous media [14]. Organic macromolecules, such as natural organic matter, polysaccharides and proteins, are ubiquitous in natural waters and therefore readily available to interact with graphene [13]. Considered molecular interactions are $\pi-\pi$ bonding and hydrophobic effects, since the mentioned macromolecules present $\mathrm{C}=\mathrm{C}$ double bonds as well as aromatic rings [17]. The concept and the preparation of graphene-based materials have been explored for over 100 years. As early as in 1859, Brodie prepared graphitic oxide with a strong oxidizing mixture; for example, nitric acid $\left(\mathrm{HNO}_{3}\right)$ with potassium chlorate $\left(\mathrm{KClO}_{3}\right)$. In 1958, Hummers reported a method to fabricate graphitic oxide [18]. However, these methods produced toxic gases $\left(\mathrm{ClO}_{2}, \mathrm{NO}_{\mathrm{x}}\right)$, and were not environmentally friendly. Many of studies related to graphene synthesis have used Hummers' method. This method uses hazards chemical during the process; for example, potassium permanganate, sulfuric acid, and sodium nitrate. Furthermore, toxic gases are released during synthesis such as $\mathrm{NO}_{2}$ and $\mathrm{N}_{2} \mathrm{O}_{4}$. These flaws led researchers to search for green methods. For example, Chen et al. excluded $\mathrm{NaNO}_{3}$ from the reaction and generated less waste during the synthesis [19]. Marcano et al. [20] has modified Hummers' method and excluded $\mathrm{NaNO}_{3}$. This method increased the reaction yield, but more $\mathrm{KMnO}_{4}$ was required [20]. As an economically and environmentally promising alternative, laser induced graphene (LIG) can be fabricated 
using one-step scalable way, reagent-free process which was reported by Lin et al. [21], by irradiation of a commercial polyimide (PI film) by a $\mathrm{CO}_{2}$ infrared laser under ambient conditions without the addition of hazardous chemicals. LIG has been studied for supercapacitors and energy storing devices [22] and recently in environmental applications. For example, an extremely low fouling surface property, and LIG electrodes were highly antimicrobial [23] and as an adsorbent to remove cationic and anionic dyes from aqueous solution [24].

\subsection{Research objectives}

The objective of this work is to investigate the colloidal stability of carbon dots and graphene oxide in natural water condition (i.e. $\mathrm{pH}$, natural organic matter, monovalent and divalent cations). Also, investigate the ability to fabricate a uniform, stable and strongly bonded laser induced graphene (LIG) layers on ceramic membranes and study the effect of solvent properties on the LIG membrane behavior. To further investigate water treatment applications, the performance of LIG to adsorb atrazine from water was evaluated.

Chapter two presents a comprehensive literature review of carbon nanomaterials. Areas covered include fabrication, properties and applications of graphene, graphene oxide, laser induced graphene and carbon dots.

In Chapter Three, we study for the first time the colloidal stability of carbon dots, an emerging carbon nanomaterial, in natural water conditions (i.e. variable salinity, $\mathrm{pH}$, dissolved organic matter) and the effect of the transformations they undergo under such circumstances on their fluorescence properties. Two types of carbon dots were considered: as obtained from thermal treatment of organic molecules, and surface functionalized, to 
represent differences in the surface chemistry. The particles were synthesized and characterized with respect to size and surface chemistry. Aggregation was investigated under environmental relevant conditions and DLVO theory applied to the modeling of particle stability. The main content of this chapter is from the following publication:

M. Bayati, J. Dai, A. Zambrana, C. Rees, M. Fidalgo de Cortalezzi, Effect of water chemistry on the aggregation and photoluminescence behavior of carbon dots, $\mathbf{J}$.

Environ. Sci. (2017). doi:http://dx.doi.org/10.1016/j.jes.2017.03.009.

In Chapter four, we investigated the effects of the GO surface physical and chemical transformations induced by the water chemistry on the aggregation behavior of the GO sheets. GO suspensions in the presence of monovalent and divalent cations at variable concentrations were studied for the full range of environmentally relevant $\mathrm{pHs}$. The role of NOM chemical composition was evaluated, selecting humic acid and tannic acid as model compounds. Experimental results were compared to predictions by classic DLVO model of colloidal interactions. GO sheets have been largely modeled as spherical particles, regardless of their recognized $2 \mathrm{D}$ geometry. However, GO is not a rigid particle and it is expected to bend and crumple when subjected to the attractive forces during aggregation, leading to an intermediate and variable dimensionality as the aggregation process progresses. The applicability of the DLVO model as a predictive tool and the geometry of the modeled system is discussed. The main content of this chapter is from the following publication:

B. Mohamed, F. de C. Maria, Aggregation of Graphene Oxide in Natural Waters: Role of Solution Chemistry and Specific Interactions, J. Environ. Eng. 145 (2019) 4019050. doi:10.1061/(ASCE)EE.1943-7870.0001561.

In Chapter five, we successfully fabricated a symmetric composite ceramic membrane with LIG effective separation layer. The LIG was extensively characterized as 
well as the parameters in the laser conversion process and the resulting membrane tested with operation tests. The permeability was studied by means of permeation experiments at operation pressure between 10 and 20 psi to investigate the effect of trans-membrane pressure on the membrane permeability. Different solvents were used to study the effect of solvent properties on the membrane behavior. The main content of this chapter is from the following publications:

M. Bayati, H. Peng, H. Deng, J. Lin, M. Fidalgo, Graphene Coated Microfiltration Ceramic Membrane Fabricated by Photothermic Conversion of Polyimide, MRS Adv. 2 (2017) 2489-2495. doi:10.1557/adv.2017.398.

M. Bayati, H. Peng, H. Deng, J. Lin, M. Fidalgo de Cortalezzi, Laser induced graphene /ceramic membrane composite: Preparation and characterization, J. Memb. Sci. 595 (2020) 117537. doi:https://doi.org/10.1016/j.memsci.2019.117537.

M. Bayati, H. Peng, M.F. de Cortalezzi, H. Deng, J. Lin, T.A. White, SEM and TEM Study of a Ceramic Membrane/Laser Induced Graphene Composite, Microsc. Microanal. 23 (2017) 1742-1743. doi:10.1017/S1431927617009370.

In Chapter six, to further investigate water treatment applications, LIG was used for the first time to adsorb atrazine commonly used herbicide in the USA. The LIG was extensively characterized and batch experiments were conducted to measure the adsorption of atrazine. The influence of different adsorption conditions was studied: solution $\mathrm{pH}$, ionic strength, adsorption time, initial adsorbate concentration. The main content of this chapter is from the following publication:

M. Bayati, M. Numaan, A. Kadhem, Z. Salahshoor, S. Qasim, H. Deng, J. Lin, Z. Yan, C.H. Lin, M. Fidalgo de Cortalezzi, Adsorption of atrazine by laser induced graphitic material: An efficient, scalable and green alternative for pollution abatement, $\boldsymbol{J}$. Environ. Chem. Eng. 8 (2020) 104407. doi:https://doi.org/10.1016/j.jece.2020.104407.

Chapter 7 is the conclusions and future works. 


\section{CHAPTER TWO}

\section{Literature Review}

\subsection{Introduction to carbon materials}

The study of carbon material can be dated back to $3750 \mathrm{BC}$, the first stated use of it being to reduce copper and zinc minerals. While it was not until the late 18 th century that carbon was identified as an element by the well-known French chemist Antoine Lavoisier [25], scientific development with respect to carbon has been verified from all over the world, for example using charcoal for therapeutic purposes, as retrieved from Egyptian papyri in about $1500 \mathrm{BC}$. Hippocrates and Pliny also used charcoal to 'treat' diseases such as epilepsy during $400 \mathrm{BC}$. Around the same time, the first records of charcoal as water filters are obtained from both Phoenician and Hindu cultures. Records of further advances have been shortly stopped, when Roman Emperor Diocletian called for destruction of scientific books in 297 AD [26].

The identification of the allotropes of carbon also follows a similar timeline, with diamond being the first to be discovered approximately 3000 years ago in India. While graphite was also 'discovered' around $4000 \mathrm{BC}$, it was then known as 'blacklead' and used for pottery decorations during Neolithic times. It was not until 1789 that German geologist Abraham Gottlob Werner actually named it graphite, meaning 'writing stone'. The third allotrope to be synthesized was Buckminsterfullerene, in 1985 by Kroto and coworkers at Rice University [27,28] This essential finding turned the attention of 
researchers towards chemically fabricating individual sheets of graphitic carbon and eventually led to the discovery of finite, carbonaceous tubular structures in 1991 by Iijima [29], which known as carbon nanotubes (CNTs). A revolution in the field of nanotechnology was initiated when few-layer 'graphene' was isolated in 2004 by Geim and Novoselov [30], reached its apex with graphene winning the Nobel Prize for Physics in 2010. Since the discovery of free-standing graphene films in 2004, a huge number of studies have followed up with experimental and theoretical evidence of exceptional optical [31], chemical and electronic properties [30]. Graphene can be a basic building block for other derivative nanomaterials: it can be rolled into carbon nanotubes (CNTs), stacked into graphite, or wrapped into fullerenes (Figure 2.1).

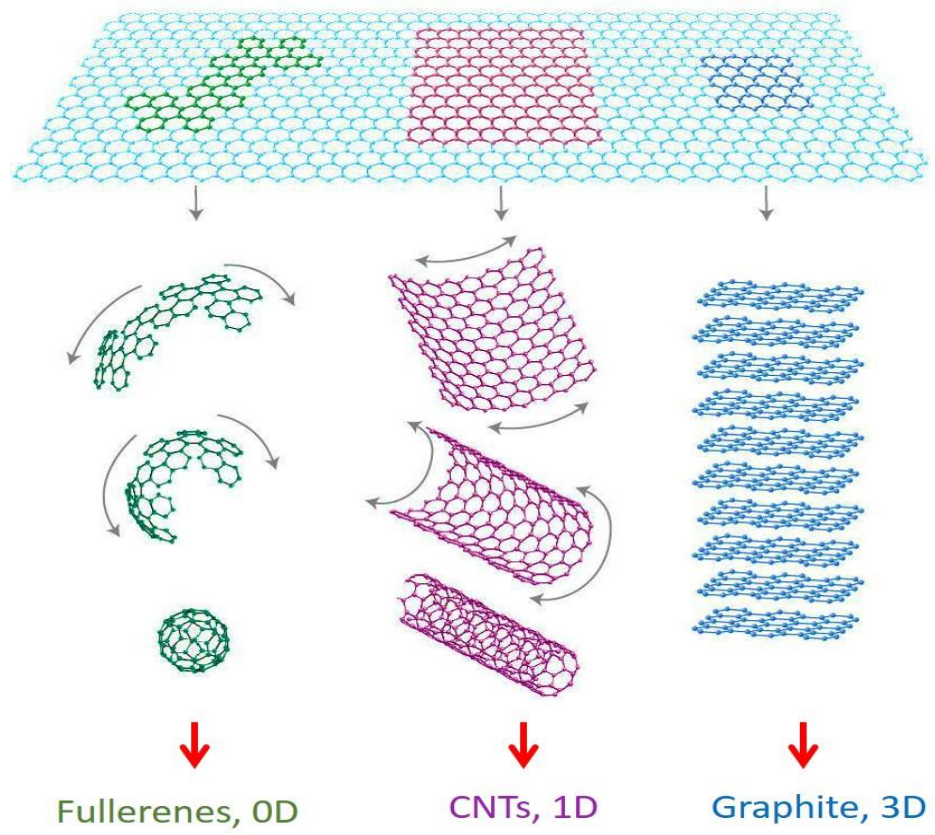

Figure 2.1 Overview of graphene-based nanomaterials. Graphene can be wrapped into OD fullerenes, rolled up into 1D nanotubes, or stacked into 3D graphite [30]. 
Figure 2.2 presents the history of graphene-based materials, the first preparation of GO in 1840 , to 2004, where graphene gained major importance.

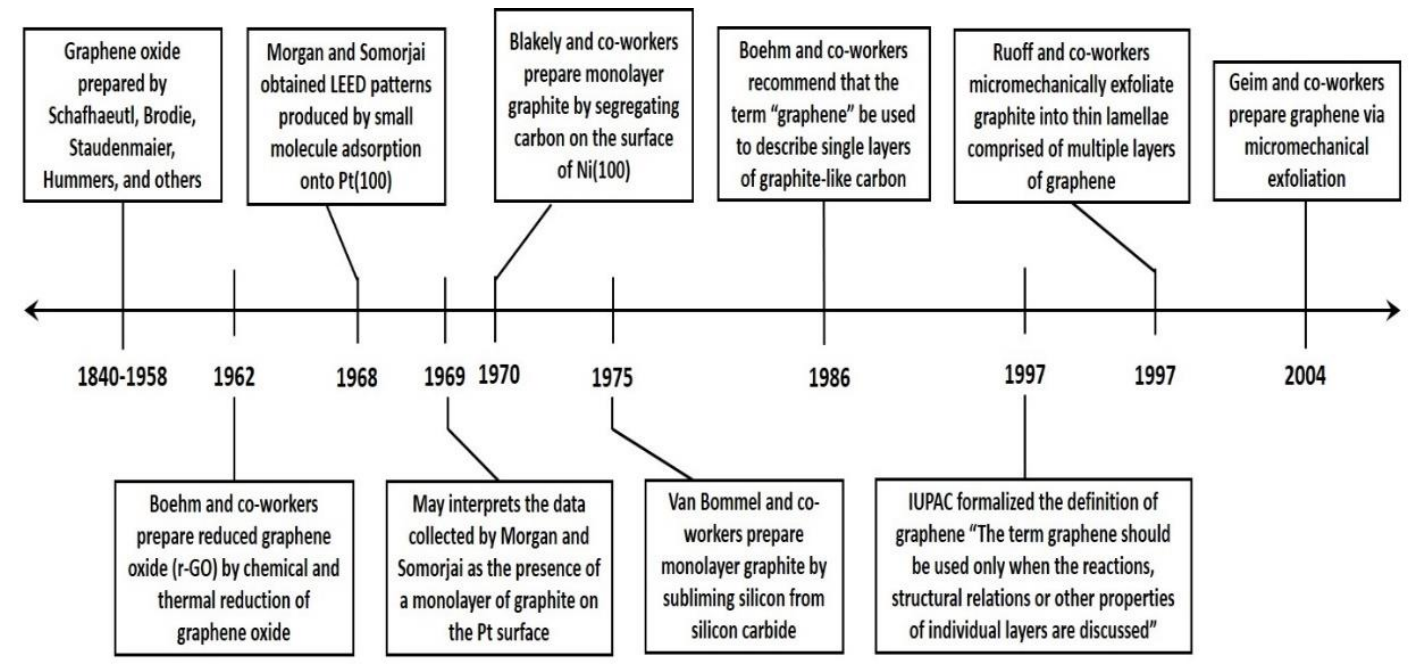

Figure 2.2 History of graphene-based nanomaterials [32].

Carbon nanomaterials are extensively used in nanoscale material sciences: from zero (carbon dots, fullerene), one (single-walled nanotube), to two dimensional nanomaterials (graphene, graphene oxide)[33]. During the past two decades, carbon was used as a substitution candidate for highly fluorescence quantum dots. Quantum dots often have a heavy metal core, thus have intrinsic toxicity and tedious preparation steps. The alternative materials are carbon dots (CDs), named after its main compositional element. Due to their biocompatible composition and straightforward synthesis, CDs have become the center of research efforts. Later, CDs were discovered to have favorable photophysical properties that are quantitatively comparable to semiconductor-based quantum dots. CDs have superior optical properties, such as two photon imaging, size dependent photoluminescence emission, photobleaching resistance, and excitationwavelength-dependent photoluminescence emission. Some of these properties are unique 
for CDs, but not for quantum dots. In addition to its advantageous optical properties, CDs are biocompatible and are capable of undergoing various surface modifications. These beneficial physical characters facilitate many applications of CDs, for example medical bone imaging, targeted drug delivery [34].

\subsection{Graphene and graphene oxide}

Graphene is a single atom thick sheet of $\mathrm{sp}^{2}$ hybridized carbon atoms arranged in a two-dimensional honeycomb lattice of six-membered rings. Graphite, a 3-dimensional (3D) layered crystal lattice structure, is formed by stacking parallel 2-dimensional (2D) graphene sheets as is shown in Figure 2.3. The distance between carbon atoms in the lattice is $0.142 \mathrm{~nm}$ (Figure 2.4), and the distance between planes is $0.335 \mathrm{~nm}$ in graphite structure $[35,36]$.

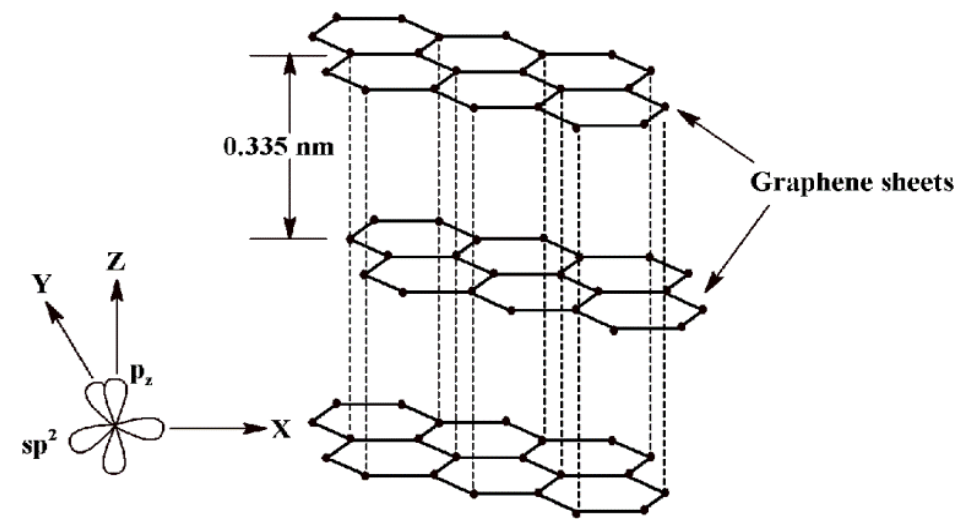

Figure 2.3 Layered structure of graphite showing the $\mathrm{sp}^{2}$ hybridized carbon atoms tightly bonded in hexagonal rings [35]. 

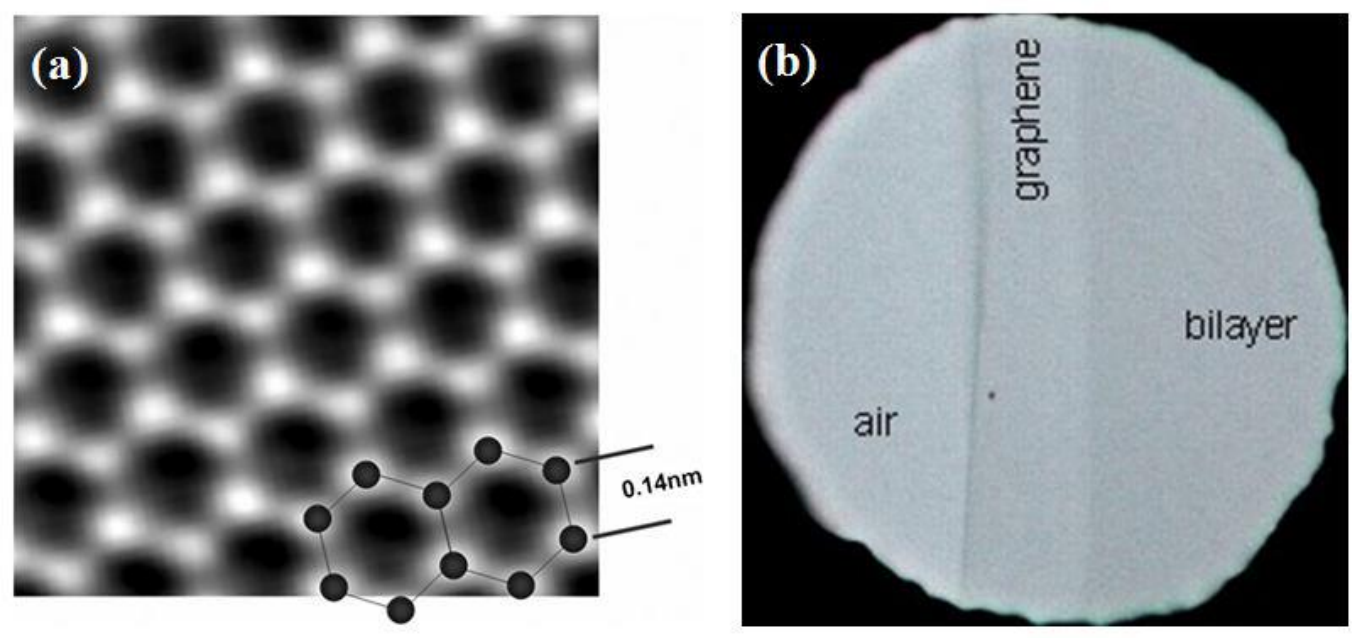

Figure 2.4 (a) HRTEM image of a graphene sheet showing the crystalline lattice. (b) A single sheet graphene can be visualized by eye [37]

Graphene has very large surface area and tends to be densely packed due to van der Waals interactions between its sheets. In general, graphene synthesis can be carried out by four different methods: Mechanical exfoliation, chemical vapour deposition (CVD), epitaxial growth and chemical reduction of graphene oxide [36,38]. Mechanical exfoliation is a simple peeling process of highly-ordered pyrolytic graphite (HOPG) by a adhesive tape, as foe example scotch tape. Thin flakes left on the tape are composed of monolayer or a few layers of graphene [31,39]. This method generates pristine graphene, but this approach is limited by its low production rate.

Chemical vapor deposition (CVD) is a substrate-based method that involves the diffusion of the carbon into a transition metal substrate at the growth temperature followed by a precipitation of carbon out of the bulk metal to metal surface upon cooling. Typically, Ni or Co films are used as substrate and methane as the feeding gas. The deposition process is carried out in a CVD chamber under vacuum at a temperature below $1000{ }^{\circ} \mathrm{C}$. Upon cooling, the solubility of the carbon on the substrate decreases and a thin 
film of carbon precipitates on the surface to form graphene sheets. The thickness of the graphene layers is controlled by the cooling rate and the concentration of carbon dissolved in the Ni substrate. However, difficulty to control film thickness and the requirement of expensive substrate materials are major disadvantages of the CVD method, that limit its application in large-scale production [36,40,41].

Epitaxial growth is another substrate-based method where a graphene monolayer is grown on Silicon carbide ( $\mathrm{SiC}$ ) by the high temperature reduction of a $\mathrm{SiC}$ substrate. High thermal treatment of $\mathrm{SiC}$ under vacuum results in sublimation of the silicon atoms and the formation of graphene by reorganization and graphitization of the carbon-enriched surface. A number of physical properties differ significantly between epitaxially grown and mechanically exfoliated graphene due to the influence of interfacial effects in epitaxial graphene $[40,41]$.

Chemical conversion of graphene into graphene oxide (GO) has become a viable route to synthesize graphene in considerable quantities. GO is usually synthesized through the oxidation of graphite using oxidants based on Hummers method [18]. GO reduction can be done by either chemical or thermal reduction approach. Chemical reduction of GO sheets has been performed with several reducing agents such as hydrazine $[42,43]$, sodium borohydride [44]. Thermal reduction utilizes heat treatment to remove the oxygen functional groups from GO surfaces [45].

GO on the other hand is the highly oxidized derivative of graphene possessing different oxygen functional groups such as hydroxyl $(\mathrm{C}-\mathrm{OH})$, carbonyl $(\mathrm{C}=\mathrm{O})$, epoxy $(\mathrm{C}$ O-C) and carboxyl (COOH). These groups found on both the edge and basal plane of the nanosheets, as shown in Figure 2.5. Nevertheless, these groups make graphene oxide 
highly attractive as a multifunctional material for a wide range of applications, as it can be readily modified with several functional groups [46].

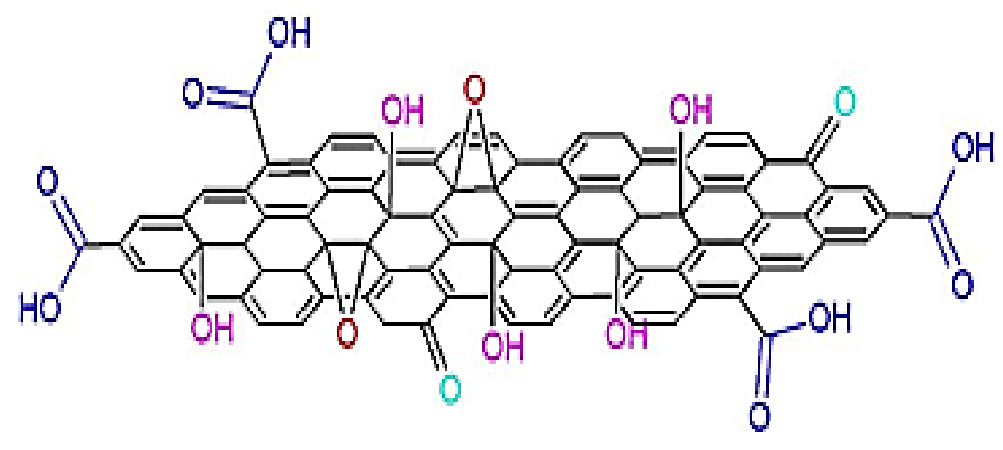

Figure 2.5 Schematic structure of graphene oxide [46].

When compared to graphite, the interlayer spacing of GO is more than twice that of its parent's materials due to functional groups covalently bonded to its basal plane. While graphite shows an interlayer spacing of $0.335 \mathrm{~nm}, \mathrm{XRD}$ analysis has reported values of $0.88 \mathrm{~nm}$ for GO [47]. In polar solvents, the functional groups are responsible for a stabilizing electrostatic repulsion effect that yields stable, hydrophilic colloidal solutions.

\subsubsection{Synthesis of GO}

Approximately 150 years ago, Brodie prepared graphite oxide through the oxidation of graphite using potassium chlorate and nitric acid mixture $[48,49]$. The Brodie method was modified by Staudenmaier, 1898 who replaced the oxidizing agent by a mixture of sulfuric acid and nitric acid [36,49]. In 1958, Hummers and Offeman stated less hazardous and more efficient method using a water-free mixture of sodium nitrate, potassium permanganate and concentrated sulfuric acid, the method most commonly used 
today [18]. Recently, Tour and co-workers improved the Hummers' method by excluding the $\mathrm{NaNO}_{3}$, increasing the amount of $\mathrm{KMnO}_{4}$, and performing the reaction in a 9:1 mixture of $\mathrm{H}_{2} \mathrm{SO}_{4} / \mathrm{H}_{3} \mathrm{PO}_{4}$. The method has advantages of its simpler protocol, higher yield, higher efficiency, fewer defects and no toxic gas evolution during oxidation [20]. Figure 2.6 shows schematic presentation of synthesis of GO [51].

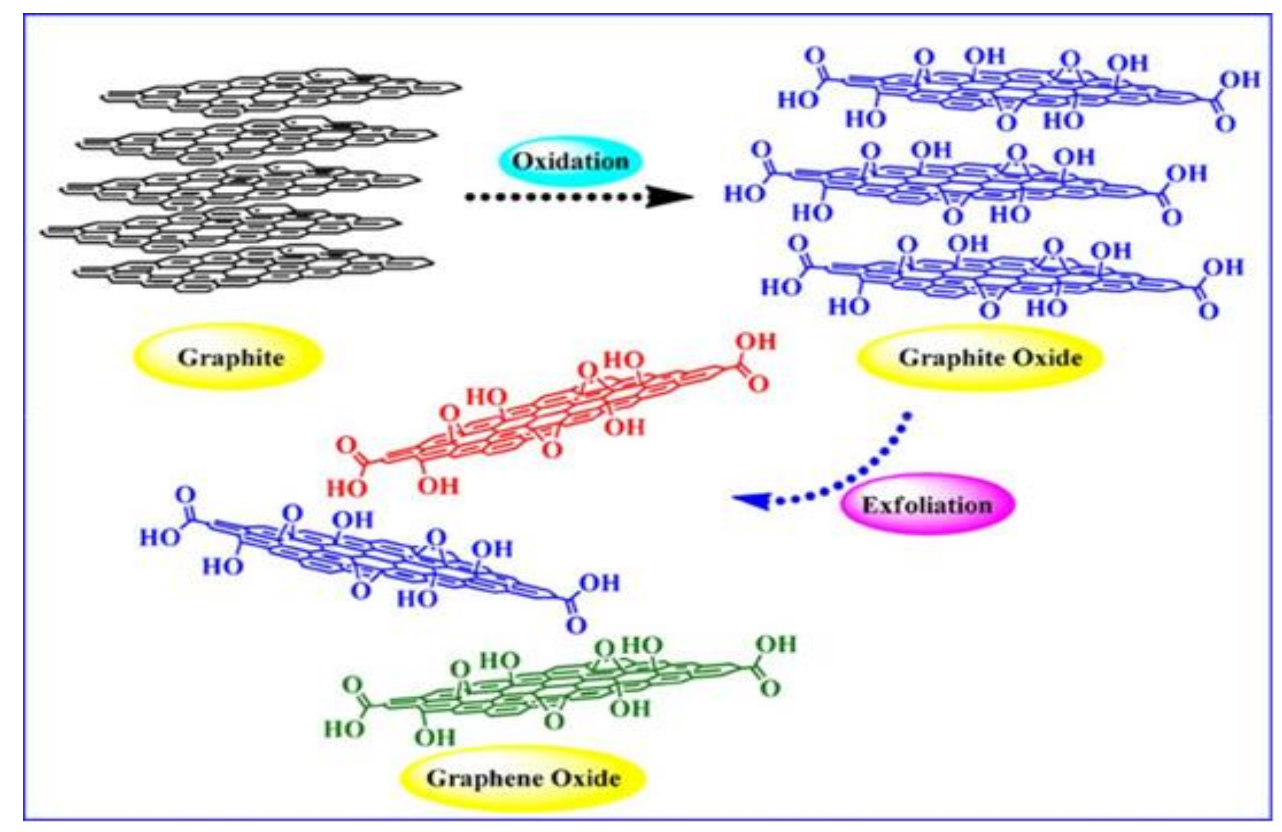

Figure 2.6 Synthesis of graphene oxide [51].

\subsubsection{Graphene nanomaterials in aquatic environments}

Graphene, graphene oxide (GO), the oxidized form of graphene and reduced graphene oxide (rGO), produced by thermal annealing or chemical treatment to eliminate oxygen-containing functional groups from GO are exhibit unique structure and remarkable physiochemical properties which result in a wide range of applications [5255]. Due to their wide use, graphene-based nanomaterials have the potential to be released into the environment from their manufacture, transport, and disposal. Recent 
studied have shown that graphene-based nanomaterials are toxic to living organisms. For instance, both GO and rGO are toxic to human umbilical vein endothelial cells [56] and bacteria [57]. Graphene has shown toxic effects on algal cells [58],bacteria [59], mammalian fibroblasts [60] and Daphnia magna [61]. The introduction of GO results in severe and persistent lung injuries [62]. Another study highlighted the effect of GO on the immune system by exposing murine macrophages, RAW cells and human whole blood cell cultures [63]. The results showed that GO was cytotoxic to both RAW and whole blood cell cultures. Therefore, GAs may pose potential environmental and human health risks if they are released into soil and groundwater systems.

The colloidal properties of graphene-based nanomaterials depend on their physicochemical properties within a given aqueous medium and are ultimately reflected in their aggregation and deposition behaviors. Thus, a comprehensive understanding of the aggregation and deposition behaviors of graphene-based nanomaterials is important not only for promoting their industrial applications but also for more precisely evaluating their environmental fate and consequently assessing their environmental impact and risks [64].

\subsubsection{Aggregation behavior of GO in aquatic environments}

The surface of GO sheets is functionalized with different oxygen containing functional groups, which allows GO sheets to form relatively stable suspensions in water. Upon release into aquatic environments, it is expected that $\mathrm{GO}$ sheets to interact with natural system constituents such as inorganic ions, natural organic matter (NOM), colloidal particles and biocolloids. Those interactions will affect the colloidal behavior of 
$\mathrm{GO}$ and determine the fate of $\mathrm{GO}$ particles in aquatic environments, as reported by several researchers [65]. This section mainly covers the colloidal behavior of GO as a function of $\mathrm{pH}$, ionic strength (IS), salt type, NOM.

\subsubsection{Influence of $\mathrm{pH}$}

The $\mathrm{pH}$ is one of the most important factors controlling the stability of GO in water because of its influence on the degree of ionization of the oxygen-containing functional groups on the surface of GO. The GO suspension exhibits very different appearances at extremely acidic $\mathrm{pH}$ values $(\mathrm{pH} 1-3)$, intermediate $\mathrm{pH}$ values ( $\mathrm{pH} 3-12)$, and extremely alkaline $\mathrm{pH}$ values $(\mathrm{pH} 12-14)$. $\mathrm{GO}$ precipitates at $\mathrm{pH}<3$ and is stably suspended in the range of $\mathrm{pH} 3-10$. Shih and coworkers [66] observed that the GO suspension was visually homogeneous with a dark-brown color at $\mathrm{pH} 14$. Taniguchi and coworkers [67] observed that the color of the GO suspension change from slight-brown to dark-brown when the $\mathrm{pH}$ value increased from 3 to 12 . They attributed this observation to the $\mathrm{pH}-$ driven reversible epoxide ring opening/closing on the GO basal plane.

The response of GO stability to varying $\mathrm{pH}$ value mainly results from the $\mathrm{pH}$-induced surface charge variation (i.e., protonation or deprotonation of a high density of oxygencontaining functional groups). At low solution $\mathrm{pH}$ values, the presence of a large number of protons may suppress the deprotonation of the carboxyl groups and reduces the hydrophilicity at the edge. Meanwhile, the intact carboxyl groups readily form carboxylic dimers through strong intermolecular hydrogen bonding [68]. As a result, GO may aggregate and settle. The carboxyl groups at the edge of GO are gradually protonated as $\mathrm{pH}$ decreases which makes $\mathrm{GO}$ sheets more hydrophobic, forming aggregates. In these 
cases, van der Waals interaction should dominate over electrostatic repulsion. In addition, the residual $\pi$-conjugation in the basal plane of GO may also contribute to the aggregation via $\pi-\pi$ stacking. Both cases would favor GO aggregation via the face-toface pattern. By employing MD simulations, Tang et al. stated that hydrophobic interaction played a dominant role in the aggregation of $\mathrm{GO}$ at low $\mathrm{pH}$ values. At basic pHs, deprotonation of carboxylic and phenolic groups can be affected, and it increases with $\mathrm{pH}$ level. As a result, electrostatic stabilization of GO in aqueous solutions can be achieved when the suspension $\mathrm{pH}$ is higher than the $\mathrm{pKa}$ of carboxyl or phenolic hydroxyl surface groups. The deprotonation of hydroxyl is much weaker than carboxyl and may not be important to the surface charge acquisition of GO [70]. Since GO have both hydrophobic and hydrophilic surface groups, the deprotonation of carboxyl groups will maximize the difference in the extent of hydrophobicity between the edge and the basal plane [66].

According to the principle that "like dissolves like", the strong hydrophilicity of carboxylic groups at the edge may pull GO into bulk water, which results in its easy dispersion in water. Besides, at higher $\mathrm{pH}$, more $\mathrm{H}$-bonds formed between $\mathrm{GO}$ sheets and water, making GO more hydrophilic and increasing affinity in water [69]. Therefore, it can be concluded that the degree of deprotonation of the edge carboxylic groups, the formation of $\mathrm{H}$-bonds and the electrostatic repulsion between the negatively charged deprotonated carboxylic groups are the driving forces for the increased stability of GO at higher $\mathrm{pH}$ values. Previous studies monitored the surface charge, size, and suspended mass concentration as a function of $\mathrm{pH}$ mainly dynamic light scattering, time-resolved dynamic light scattering, and UV-vis spectroscopy, respectively [71]. The zeta potential 


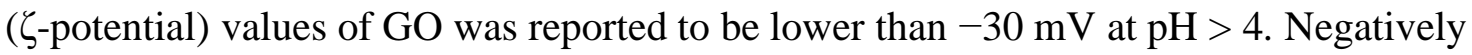
charged colloids with $\zeta$-potential more than $-30 \mathrm{mV}$ are generally considered to be stable and well dispersed in water [72,73]. Therefore, GO sheets are stable at $\mathrm{pH}>4$. The change in $\zeta$-potential and hydrodynamic diameters $\left(D_{h}\right)$ of GO as a function of $\mathrm{pH}$ showed a similar trend [74].

\subsubsection{Influence of IS and salt type}

Surface water and groundwater environments contain a complex mixture of inorganic ions such as $\mathrm{Na}+, \mathrm{K}^{+}, \mathrm{Mg}^{2+}, \mathrm{Ca}^{2+}, \mathrm{Cl}^{-}, \mathrm{HCO}_{3}{ }^{-}, \mathrm{SO}_{4}{ }^{2-}$, etc., which varies with location and can have a significant impact on the electro-kinetic behavior of colloids and hence their colloidal properties [75]. Ions with an opposite charge to the surface charge of colloids, namely counter-ions, tend to accumulate at the charged interface, compress the electrostatic double layer (EDL), and effectively reduce the energy barrier between colloids by neutralizing the particle surface charge. This results in the attractive van der Waals interactions becoming dominant and increasing the particle aggregation [76]. Charge neutralization can also occur as a result of specific adsorption of counter-ions on the charged surface. The degree of charge neutralization is dependent on the electrolyte concentration, valence of counter-ions, and affinity between the electrolyte and colloids. Surface charge screening is directly reflected in the electrophoresis mobility (EPM) and $\zeta$-potential of the colloids. With an increase in the counter-ion concentration, the absolute values of EPM and $\zeta$-potential approach zero, indicating a favorable aggregation condition. The effects of the common environmental cations $\left(\mathrm{Na}^{+}, \mathrm{K}^{+}, \mathrm{Mg}^{2+}\right.$ and $\left.\mathrm{Ca}^{2+}\right)$ on 
the surface potential of GO are well established. The cations increase the surface charge of colloids in the order $\mathrm{K}^{+}>\mathrm{Na}^{+}$for monovalent counter-ions $[76,77]$ and $\mathrm{Ca}^{2+}>\mathrm{Ba}^{2+}>$ $\mathrm{Mg}^{2+}$ for divalent counter-ions [77]. Qi et al. attributed the greater aggregation effect of $\mathrm{K}^{+}$than $\mathrm{Na}^{+}$to the less dense hydration of $\mathrm{K}^{+}$, and the higher aggregation effect of $\mathrm{Ca}^{2+}$ than $\mathrm{Mg}^{2+}$ to the less dense hydration and the bridging effect of $\mathrm{Ca}^{2+}$.

Besides neutralizing surface charges, divalent electrolytes which can specifically interact with GO to cross-link them are much more effective in destabilizing GO suspensions. Wu et al. proposed that $\mathrm{Ca}^{2+}$ and $\mathrm{Mg}^{2+}$ induced the aggregation of $\mathrm{GO}$ sheets through the following three types of cross-linking interactions: (1) bridging the edges of the GO sheets through chelating carboxylate groups, (2) intercalating between the basal planes through either weak alkoxide or dative bonds from carbonyl and hydroxyl groups, and (3) cross-linking of the H-bonds formed among the oxygen containing functional groups on GO surfaces and the interlamellar water molecules. Specially, preferential adsorption plays an important role in the destabilization of GO by $\mathrm{Ca}^{2+}$. Chowdhury et al. observed that the $\zeta$-potential values of GO as a function of IS were quite similar for both $\mathrm{CaCl}_{2}$ and $\mathrm{MgCl}_{2}$, but the hydrodynamic sizes of $\mathrm{GO}$ as a function of IS were significantly different for $\mathrm{CaCl}_{2}$ and $\mathrm{MgCl}_{2}$. They attributed the higher effectiveness of $\mathrm{Ca}^{2+}$ in destabilizing $\mathrm{GO}$ than $\mathrm{Mg}^{2+}$ to the preferential adsorption of $\mathrm{Ca}^{2+}$ through the binding of $\mathrm{Ca}^{2+}$ with oxygen containing functional groups available on GO surfaces. Similarly, Wu et al. attributed the lower critical coagulation concentration (CCC) of $\mathrm{GO}$ in $\mathrm{Ca}^{2+}$ than that in $\mathrm{Mg}^{2+}$ to the higher propensity of $\mathrm{Ca}^{2+}$ to form complexes with carboxylated groups. 


\subsubsection{Influence of NOM}

Macromolecular NOM with various carbon functionalities (e.g., carbonyl, carboxyl, acetal, aromatic, heteroaliphatic, and aliphatic carbons), ubiquitous in natural aquatic systems, is known to function as a stabilizing agent for colloidal suspensions. Typically, the concentration of NOM is in the range from 0.1 to $2 \mathrm{mg} \mathrm{L}-1$ in ground waters and as high as $20 \mathrm{mg} \mathrm{L}^{-1}$ in surface water.30 Humic substances, as typical NOM analogues, can be operationally classified into fulvic acid (FA), which is soluble at all $\mathrm{pH}$ levels and humic acid (HA), which is soluble in alkaline aqueous solutions and insoluble in acidic aqueous solutions. NOM and its analogues can be adsorbed on GO via $\pi-\pi$ interaction, Lewis acid-base interaction, hydrogen bonding, and/or covalent and electrostatic interaction $[78,79]$. The exact interaction mechanism of GO with NOM will depend on both GO characteristics and functional derivatization as well as NOM characteristics including size, aromaticity, and charge density. NOM consists of hydrophilic side chains (carboxylic and hydroxyl groups), a hydrophobic backbone, and a large number of crosslinked aromatic rings. They show a high tendency to pack parallel to the surface of GO by strong $\pi-\pi$ interactions or to be readily adsorbed onto the basal plane of GO through hydrophobic interaction. The abundant carboxyl and hydroxyl groups from the adsorbed NOM protrude into the water and make the GO surface more hydrophilic. Suwannee River humic acid (SRHA) has been widely employed as a model to investigate the impact of NOM on GO stability. It is well established that the addition of SRHA reduces the aggregation of $\mathrm{GO}$ in $\mathrm{NaCl}, \mathrm{MgCl}_{2}$ and $\mathrm{CaCl}_{2}$ solutions. Jiang et al. reported that the addition of $1.7 \mathrm{mg} \mathrm{L}^{-1}$ Aldrich humic acid (AHA) produced an increase of the CCC from 67 to $220 \mathrm{mM}$ for $\mathrm{NaCl}$ and 1.4 to $2.2 \mathrm{mM}$ for $\mathrm{CaCl}_{2}$. Chowdhury et al. reported that the 
addition of $5 \mathrm{mg} \mathrm{L}^{-1}$ SRHA increased the CCC of GO from 44 to $125 \mathrm{mM}$ for $\mathrm{NaCl}$, from 1.2 to $3.9 \mathrm{mM}$ for $\mathrm{MgCl}_{2}$, and from 0.8 to $2.2 \mathrm{mM}$ for $\mathrm{CaCl}_{2}$. Accordingly, the significance of stability enhancement by HA is largely dependent on the concentration and the type of background electrolyte.

Generally, when the background cation is $\mathrm{Na}^{+} / \mathrm{K}^{+}$at a low concentration, the stability enhancement effect of NOM is not significant. In this case, NOM has a negligible effect on the EPM and hydrodynamic diameter $\left(D_{h}\right)$ of GO. When the background cation is $\mathrm{Na}^{+} / \mathrm{K}^{+}$at a high concentration, the stability enhancement effect of NOM is very significant. In this case, NOM has no obvious effect on the EPM of GO while it decreases the $D_{h}$ of GO, so NOM stabilizes GO via steric repulsion. When the background cation is $\mathrm{Na}^{+} / \mathrm{K}^{+}$with an extremely high concentration, NOM may fail to inhibit the aggregation of GO. In a solution containing $31.6 \mathrm{mM} \mathrm{KCl}$, the presence of $1 \mathrm{mg} \mathrm{L}^{-1} \mathrm{SRHA}$ was found to significantly decrease the $D_{h}$ of GO from $1598.1 \pm 105.1$ to $573.7 \pm 56.1 \mathrm{~nm}$. At $20 \mathrm{mM}$ $\mathrm{NaCl}$, the presence of tannic acid (TA) was reported to suppress the GO aggregation; meanwhile at $200 \mathrm{mM} \mathrm{NaCl}$, the adsorption bridging effect of TA was more significant than its steric effect, which leads to TA failing to inhibit the aggregation of GO via the formation of GO-TA-GO aggregates (Figure 2.7).
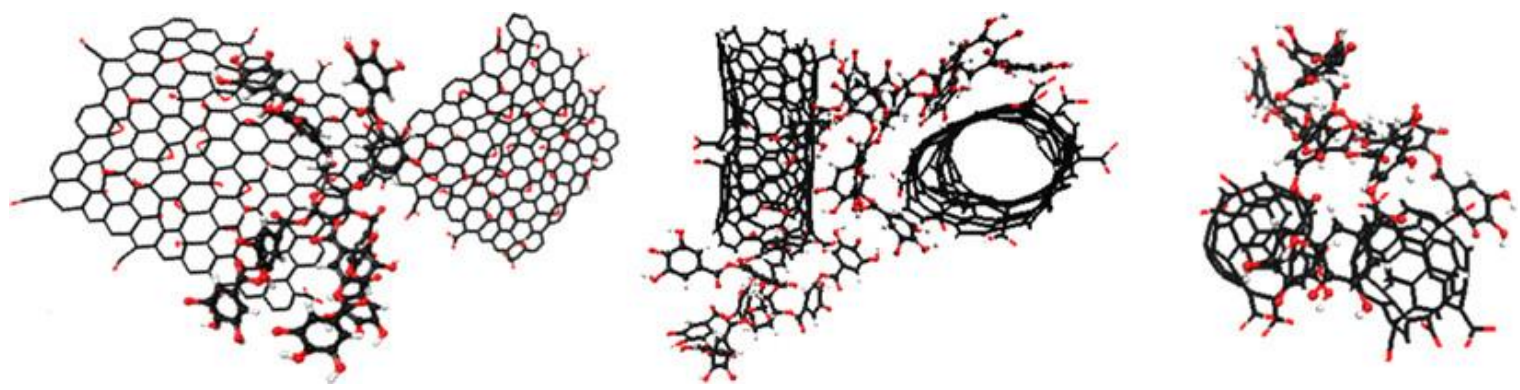

Figure 2.7 Configurations of the graphene-tannic acid-graphene aggregates [69]. 
When the background cation is $\mathrm{Ca}^{2+} / \mathrm{Mg}^{2+}$, NOM is less effective in stabilizing GO or even facilitates the aggregation of GO. In this case, two opposing processes govern the interactions of NOM with GO. First, NOM can bind with GO, which provides steric repulsion and increases the stability of GO. Second, NOM can facilitate binding with GO functional groups in the presence of divalent $\left(\mathrm{Ca}^{2+}, \mathrm{Mg}^{2+}\right)$ cations, which can increase the aggregation of GO and reduce its stability. Besides the concentration and type of cations, the solution $\mathrm{pH}$ value also affects the stabilizing efficiency of SRHA. Hua et al. observed that in the presence of SRHA with $\mathrm{Mg}^{2+}$, both the EPM and $D_{h}$ of GO decreased as the $\mathrm{pH}$ value increased from 5 to 7 and increased as the $\mathrm{pH}$ value further increased from 7 to 9. Accordingly, in the presence of $\mathrm{Mg}^{2+}$, SRHA can enhance the GO stability in an acidic solution and reduce the GO stability in an alkaline solution. The enhancement is due to the steric hindrance of HA surpassing the EDL compression induced by $\mathrm{Mg}^{2+}$. The reduction is due to the desorption of HA which weakens the steric hindrance and electrostatic repulsion. Meanwhile, SRHA cannot stabilize GO in a solution containing $\mathrm{Ca}^{2+}$ at $\mathrm{pH} 5-9$ [81]. This is because higher $\mathrm{pH}$ would accelerate HA deprotonation and facilitate the bridging effect of $\mathrm{Ca}^{2+}$ on the $\mathrm{HA}$ adsorbed on the $\mathrm{GO}$ surface.

\subsection{Carbon dots}

CDs constitute a class of quasi-spherical, photoluminescence carbon nanoparticles with sizes below $10 \mathrm{~nm}$ [1]. Compared to traditional semiconductor Q-dots and organic dyes, photoluminescent CDs are superior in terms of aqueous solubility, robust chemical inertness, easy functionalization, high resistance to photobleaching, low toxicity and 
good biocompatibility [1]. As a result, much attention has been paid to their potential application in biological labeling, bioimaging, and drug delivery [82]. During the past few years, much progress has been achieved in the synthesis, properties and application of CDs [83,84]. Several low cost and environmentally benign chemical approaches have been developed for the preparation of carbon dots $[85,86]$. Due to their strong and tunable photoluminescence, CDs have been successfully applied in sensing, energy conversion and catalysis.

As interest for both fundamental research and applications has surged dramatically, it is inevitable CDs will find their way to natural water sources where, unlike the more well studied fullerenes and carbon nanotubes, their environmental impact and fate is mostly unknown. Besides, the presumed negligible environmental impact of carbon dots can only be confirmed if their behavior in natural systems is fully understood. However, the colloidal stability of CDs in environmentally relevant aqueous suspensions has not been investigated, and neither the effect of those conditions on their PL properties.

Due to their small size and large surface area, adsorption processes are expected to play a key role in determining the physicochemical properties of CDs [9]. Adsorption may alter the surface characteristics of the nanoparticles so that they become dispersed and thus mobile in aqueous media [9][14]. Natural organic matter (e.g. humic acid) and exopolymeric substances (e.g. proteins and polysaccharides) have been shown to adsorb onto carbon nanotubes and act as stabilization agents [13]; these macromolecules can potentially interact with CDs surfaces by $\pi-\pi$ bonding and hydrophobic effects, due to the presence of $\mathrm{C}=\mathrm{C}$ double bonds as well as aromatic rings [17]. 
It has been recognized that our knowledge on nanoparticle environmental behavior needs to be continuously revised and extended to account for new materials or novel commercial applications as they emerge in order to accurately assess the associated risks [87].

\subsubsection{Synthesis of CDs}

\subsubsection{Top-Down methods}

CDs were first discovered using a top-down method in 2004 [88]. Candle soot was arc-discharged to produce single-walled carbon nanotubes (SWCNTs), and then was oxidized with nitric acid to improve hydrophilicity. SWCNTs were then extracted with basic solution. The black suspension was purified using gel-electrophoresis and resulted in two black bands (long and short tubular SWCNTs) and one fast moving fluorescent band. The fluorescent band was later isolated using molecular weight cut off (MWCO) Centric on filtration devices into three samples, and they emitted green-blue, yellow, and orange fluorescence upon excitation at $365 \mathrm{~nm}$ in order of their elution and increasing sizes. These fluorescence carbon-based materials were later named CDs due to their sizes (lateral size: $18 \mathrm{~nm}$ and height around $1 \mathrm{~nm}$ by AFM). Quantum yield of the yellow fluorescent segment was measured to be 0.016. FTIR suggested the presence of carboxyl functional groups on the surface of CDs. Furthermore, FTIR showed no C-H out-ofplane bending, which means the fluorescence was not originated from polyaromatic hydrocarbons. Energy dispersive X-ray spectroscopy elemental analysis indicated that the surface layers of CDs contain $53.93 \% \mathrm{C}, 1.20 \% \mathrm{~N}, 2.56 \% \mathrm{H}$, and $40.33 \% \mathrm{O}$. 
Even though the discovery of CDs was accidental, the topic of CDs has gained spectacular attention from scientists. Arc-discharge is not a readily available apparatus for many researchers, thus different physical approaches (Figure 2.8), such as laser ablation [89], electrochemical etching [90,91], and electrochemical oxidization [92], were explored to optimize and ease the CDs synthesis. These top-down physical approaches break down of larger carbon source, like candle soot [93], gas burner soot [94], to more materials, such as graphite rods [95], ammonium citrate salt [96], phenol/formaldehyde resin [97], polyethylene glycol (PEG) [98], to produced CDs that have excitation wavelength dependency.

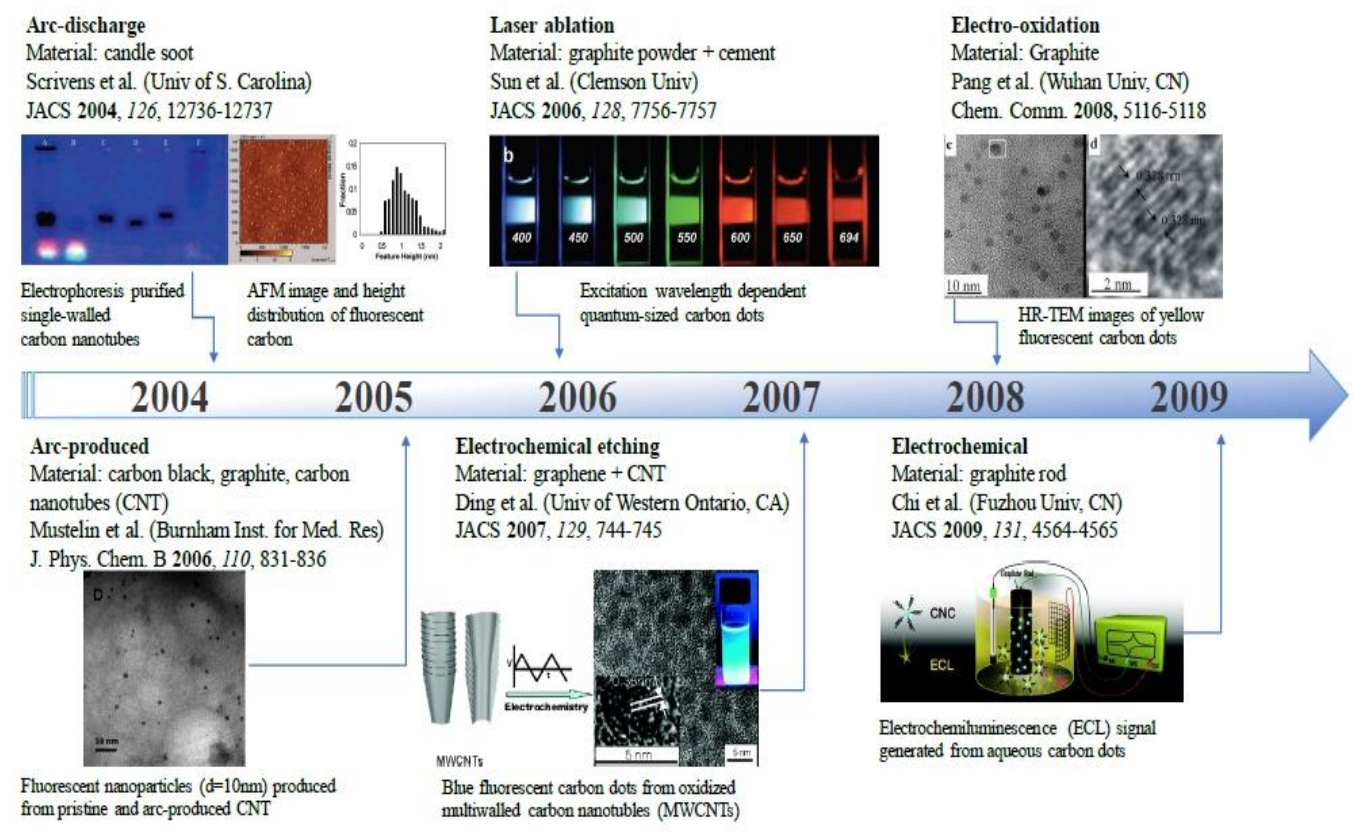

Figure 2.8 A graphic representation of top-down synthetic approaches of CDs

\subsubsection{Bottom-Up methods}

Even though CDs were discovered using top-down methods, bottom-up synthesis routes were developed to better control product simplify functionalization, uniformity, 
and lower cost. Unlike top-down methods, bottom-up synthesized CDs are often merely based on its chemistry, and utilize carbon molecular precursors, which later become part of the infrastructure/core structure of CDs. The sample dispersibilities are improved significantly, both in aqueous solution and organic solvents. Most of bottom-up synthesis use relatively mild experimental conditions, compared to top-down methods. A few representative examples are shown in Figure 2.9.

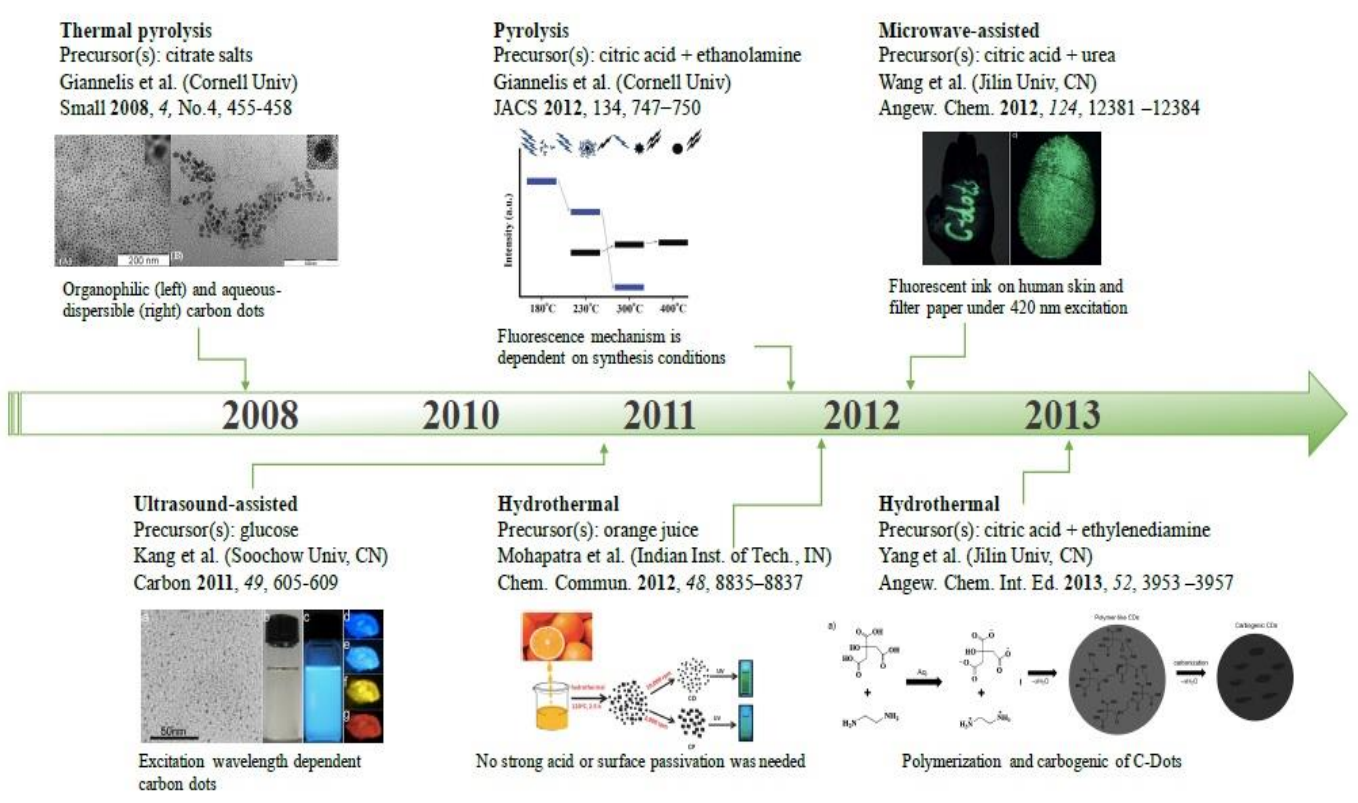

Figure 2.9 A graphic representation of bottom-up synthetic approaches of CDs

\subsection{Laser induced graphene}

In different applications, graphene is engineered into three-dimensional (3D) porous structures to provide high surface area to preserve its high mobility and mechanical stability [99-102]. Conventional approaches for the fabrication of 3D graphene include the assembly of graphene oxide (GO) into a foam. However, this approach needs the GO precursor to be prepared through its oxidative acid synthesis route $[99,100]$. Chemical 
vapor deposition (CVD) on porous substrates also produces 3D graphene, but the high temperature conditions and subsequent etching and drying process may reduce its scalable production [101,102].

In 2014, using a commercial laser scribing system, similar to that found in machine shops, a direct treatment of polyimide (PI) plastic films by the laser at room temperature and atmosphere, converted the PI into 3D porous graphene, a material termed laserinduced graphene (LIG). This one-step method does not need high temperature reaction conditions, solvent, or subsequent treatments, which results in an advantage over conventional methods for synthesis of 3D graphene [103].

\subsubsection{LIG formation on PI under ambient conditions}

The conversion of PI to LIG is a photothermal process that is associated with the localized high temperature and pressure produced by laser irradiation (Figure 2.10 a-c) $[21,104]$. The hierarchical structure and the presence of abundant wrinkles on the graphene surface (Figure 1d) produced a surface area of $\sim 340 \mathrm{~m}^{2} / \mathrm{g}$, which is comparable to that of the wet-chemistry derived 3D graphene [21]. The aberration-corrected scanning transmission electron microscopy (Cs-STEM) images (Figure 2.10 e-f) showed an unusual polycrystalline feature of LIG. Instead of the conventional hexagon lattice, the carbon of LIG existed in a hexagon and pentagon-heptagon hybrid lattice (Figure $2.10 \mathrm{f}$ ). This could be termed "kinetic graphene" since there is no time for equilibration to the standard hexagon lattice due to the rapid cooling following laser irradiation. The lasing parameters can affect the chemical and physical properties of LIG [21,105]. In general, increasing the laser power can increase the LIG thickness and improve the conductivity. 
The morphology of LIG can also be controlled by the image density, which is guided by the pulse width modulation or pulses per inch (PPI), and the lines per inch (LPI) [105].At an image density of 1000 PPI $\times 1000$ LPI and a $\sim 100 \mu \mathrm{m}$ laser spot size, the LIG will adopt an in-plane porous structure (Figure $2.10 \mathrm{c}$ ). As the image density decreases to 500 PPI $\times 500$ LPI with a $\sim 60 \mu \mathrm{m}$ laser spot size, the LIG starts to produce out-of-plane fibers (LIGF) and form a vertically aligned forest morphology (Figure $2.10 \mathrm{~g}-\mathrm{i}$ ).
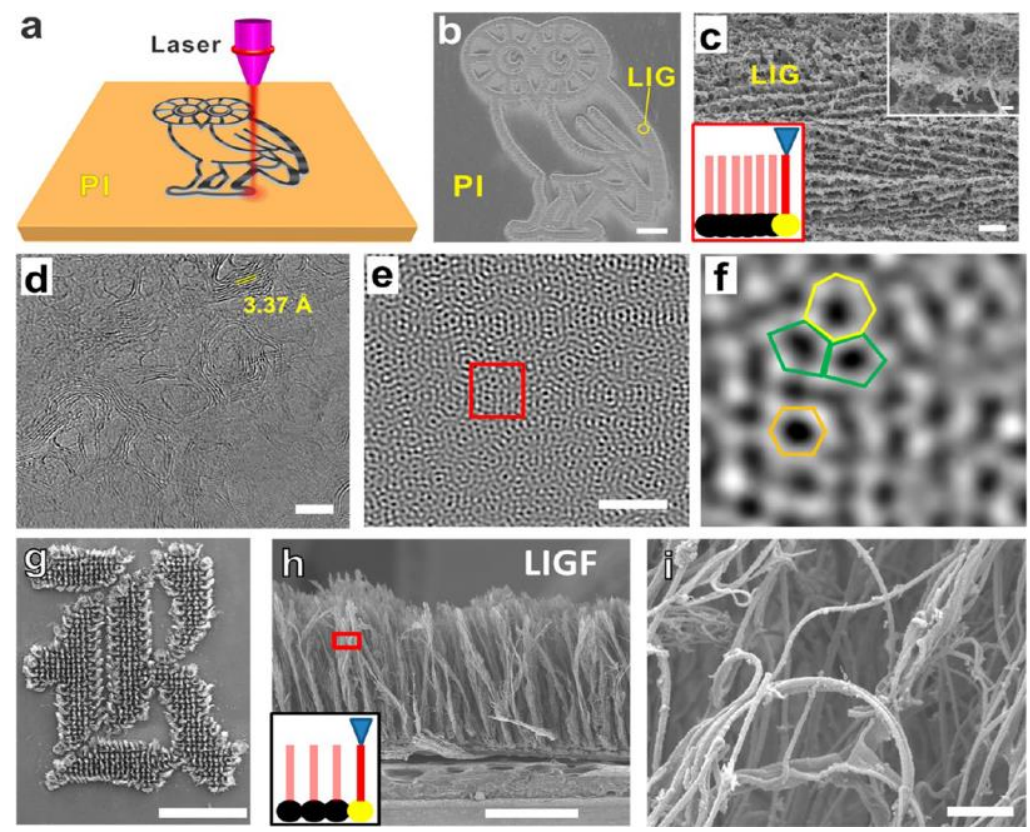

Figure 2.10 LIG formation. (a) Schematic of the synthesis process of LIG from PI. (b) SEM image of LIG patterned into an owl shape at an image density of 1000 PPI. (c) SEM image of

LIG film. (d) HRTEM image of LIG representing the average lattice space of $\sim 3.4 \AA$ corresponds to the (002) planes of graphitic materials. (e) Cs-correction STEM image taken at the edge of a LIG flake; scale bar, $2 \mathrm{~nm}$. (f) TEM image of selected area indicated as a rectangle in (e). (g) LIGF patterned in the shape of an R letter; scale bar is $1 \mathrm{~mm}$. (h) LIGF with thickness of $\sim 1 \mathrm{~mm}$ made at an image density of $500 \mathrm{PPI}$; scale bar is $500 \mu \mathrm{m}$. Inset is the schematic 500 PPI. (i) SEM of LIGF (boxed in h) at higher magnification; scale bar is $2 \mu \mathrm{m}$ [103]. 


\subsubsection{LIG formation on modified PI and phenolic resin}

The modification of the graphene composition by heteroatom doping or formation of hybrid material presents an effective approach to engineer the properties and functions of nanomaterials $[100,106,107]$. Conventional methods for tuning the composition of graphene include a CVD process which performed at high temperature [108] and a wetchemistry process that includes a synthesis route to GO, nanoparticle deposition, and GO reduction [107][100]. Using the direct laser on modified PI (m-PI) under ambient conditions for in situ modification of graphene composition could be an advantage.

Figure 2.11 a shows the preparation steps of m-PI, including the mixing of poly(amic acid) (PAA) with additives in N-methyl-2-pyrrolidone, the evaporation of the $\mathrm{N}$-methyl-

2-pyrrolidone solvent, and the dehydration of PAA to form PI. The m-PI is then subjected to laser irradiation to create LIG with varying compositions. For instance, boron doped LIG (B-LIG) is formed when m-PI is prepared from PAA containing boric acid (Figure $2.11 \mathrm{~b}-\mathrm{d}$ ). By changing the additive to metal complexes, LIG-based materials containing various metal oxide nanoparticles with uniform size distribution can be prepared (Figure $2.11 \mathrm{e}-\mathrm{h})$. 


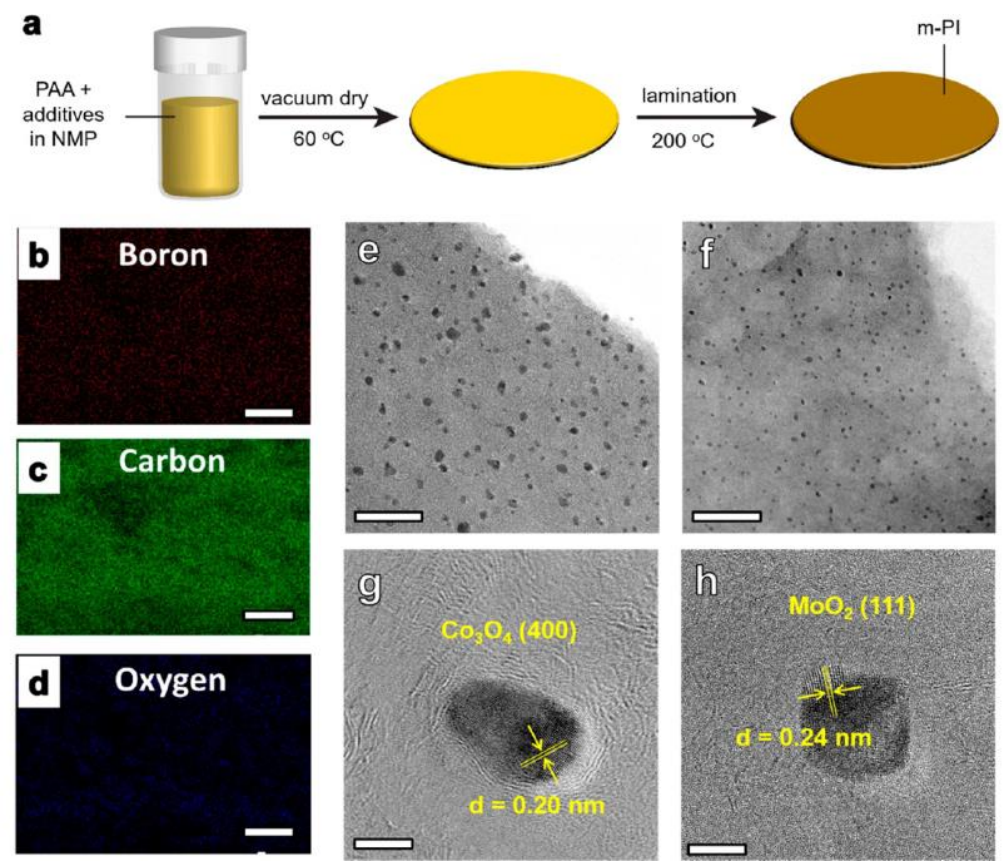

Figure 2.11 In situ formation of LIG composite. (a) The preparation steps of m-PI. Energydispersive X-ray spectroscopy of B-LIG for (b) boron, (c) carbon, and (d) oxygen. TEM images of LIG containing new uniformed sizes of $(e, g) \mathrm{Co}_{3} \mathrm{O}_{4}$ and $(\mathrm{f}, \mathrm{h}) \mathrm{MoO}_{2}$ crystalline nanoparticles [109].

\subsubsection{LIG formation on PI under controlled atmosphere}

Whereas the formation of LIG under ambient conditions provides a facile synthesis route [21], lasing under a controlled atmosphere has led to changes in surface morphology and chemical composition of the LIG, resulting in tunable hydrophobicity and hydrophilicity of the LIG surface [110]. Figure 2.12 a represents the lasing scheme with a photograph of the apparatus shown in Figure $2.12 \mathrm{~b}$. Using the laser under controlled atmosphere allows the formation of LIG under various gas environments such as $\mathrm{O}_{2}, \mathrm{Ar}$ and $\mathrm{SF}_{6}$, which mimics the traditional CVD process for the growth and modification of graphene without the need of high-temperature oven heating [111]. It is 
found that lasing on PI under ambient or an oxidizing atmosphere leads to a superhydrophilic surface (contact angle $\sim 0^{\circ}$, Figure $2.12 \mathrm{c}-\mathrm{e}$ ), while a superhydrophobic surface (contact angle $>150^{\circ}$ ) can be obtained when inert or reducing gas is applied (Figure $2.12 \mathrm{f}-\mathrm{g}$ ) [110]. The variation of superhydrophobicity is found to correlate with the LIG surface oxygen content and morphology. The decrease in oxygen content and the formation of carbon nanoparticles on the surface will both enhance the hydrophobicity. The superhydrophobicity can be further enhanced by in situ fluoro-doping when PI is lased in a chamber charged with $\mathrm{SF}_{6}$ (Figure $2.12 \mathrm{~h}$ ) [110].

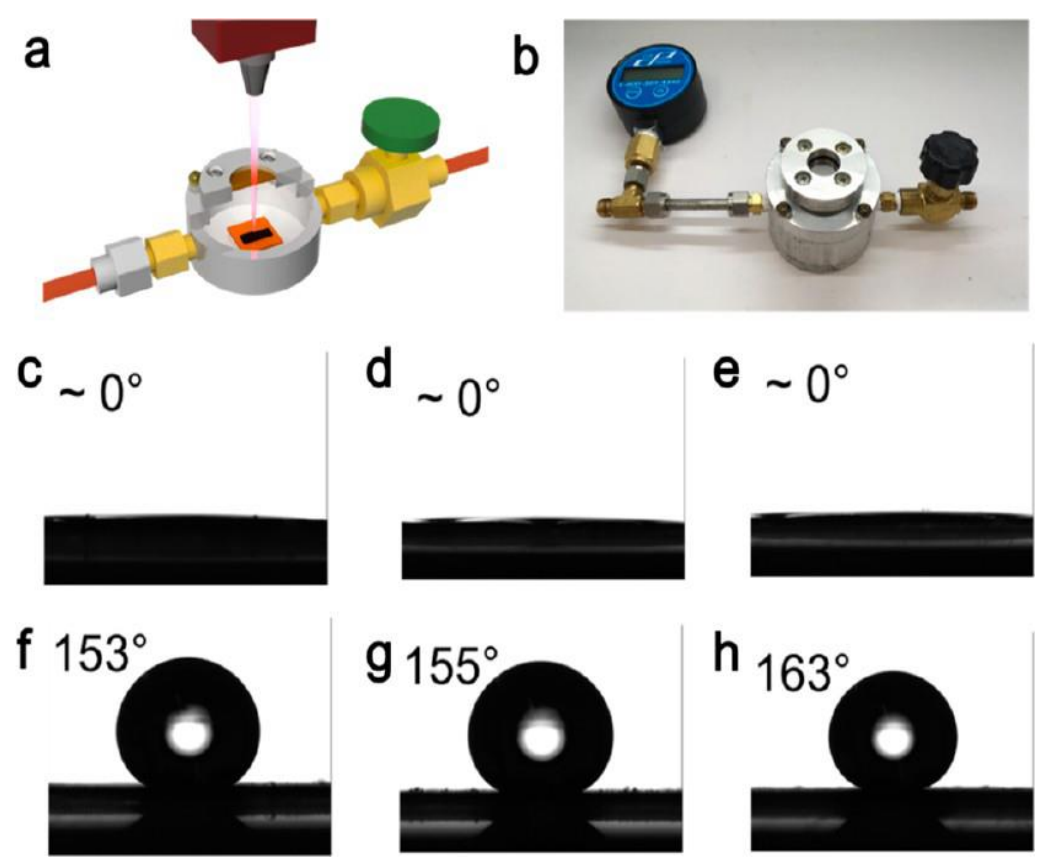

Figure 2.12 LIG formation under controlled atmosphere. (a) Scheme and (b) photograph of the apparatus for the fabrication of LIG under a controlled atmosphere. Contact angles of LIG prepared in (c) ambient air, (d) air (chamber), (e) $\mathrm{O}_{2}$ (chamber), (f) $\mathrm{Ar}$ (chamber), (g) $\mathrm{H}_{2}$ (chamber), and (h) $\mathrm{SF}_{6}$ (chamber) [110]. 


\subsubsection{Applications of LIG}

Since its finding, LIG has been extensively studied and developed for applications including electronic devices, microfluidic systems, catalysis systems, biosensor and water purification systems [111-114]. In general, there are three ways to explore its applications, which includes the direct use of pristine LIG [113,114], the formation of LIG composite in situ [115], and the deposition of active materials ex situ [22,116]. Flexible microsupercapacitors for energy storage have been developed from LIG using direct writing the interdigitated in plane electrodes on PI sheet by two approaches. The first is based on the double layer capacitive behavior of graphene carbon $[21,109]$ and the second is based on the pseudocapacitive behavior of graphene-based hybrid materials [22]. LIG also used as oxygen reduction reaction electrocatalysts. The in-situ formation of metal oxide nanoparticles embedded in LIG improved the oxygen reduction reaction (ORR) activity of LIG [115]. The enhancement benefits from the high surface area of LIG and the facilitation of electron transfer from electrode to metal oxide nanoparticles. LIG was also used for overall water splitting. The ex situ electrodeposition method offers an efficient way to expand the deposited materials on LIG beyond oxides [112,116]. Here, cobalt phosphorus $(\mathrm{Co}-\mathrm{P})$ and nickel-iron hydroxides $(\mathrm{NiFe})$ were electrodeposited onto the LIG surface to work as the hydrogen evolution reaction (HER) electrode and oxygen evolution reaction (OER) electrode respectively.

LIG was employed as a membrane for separation, taking advantage of its superhydrophobicity property $[110,117]$. The LIG filter was prepared by first producing $\sim 100 \mu \mathrm{m}$ holes in the PI sheet at high laser power and then lasing the PI in an Ar atmosphere to afford a superhydrophobic surface (Figure $2.13 \mathrm{a}-\mathrm{c}$ ). Figure $2.13 \mathrm{~d}-\mathrm{f}$ 
demonstrates the experiment of water-oil separation. The superhydrophobic LIG membrane can also be used for desalination with an air gapped distillation process as discussed by [117]. Another potential use of LIG for water treatment is the prevention of microbial fouling and the exertion of antimicrobial action (Figure 2.14) [23]. Pseudomonas aeruginosa was used as the species for biofilm study. Both living P. aeruginosa and extracellular polymeric substance (EPS) were rarely observed on the LIG surface (Figure $2.14 \mathrm{a}$ and d). However, both control samples, PI and graphite, are clearly covered by a biofilm (Figure $2.14 \mathrm{a}^{-} \mathrm{c}$ ). The antifouling property of LIG benefits from its zeta potential and the hydrophilic surface [23]. It also might arise from its superhydrophilic properties where water is not easily displaced from the surface.
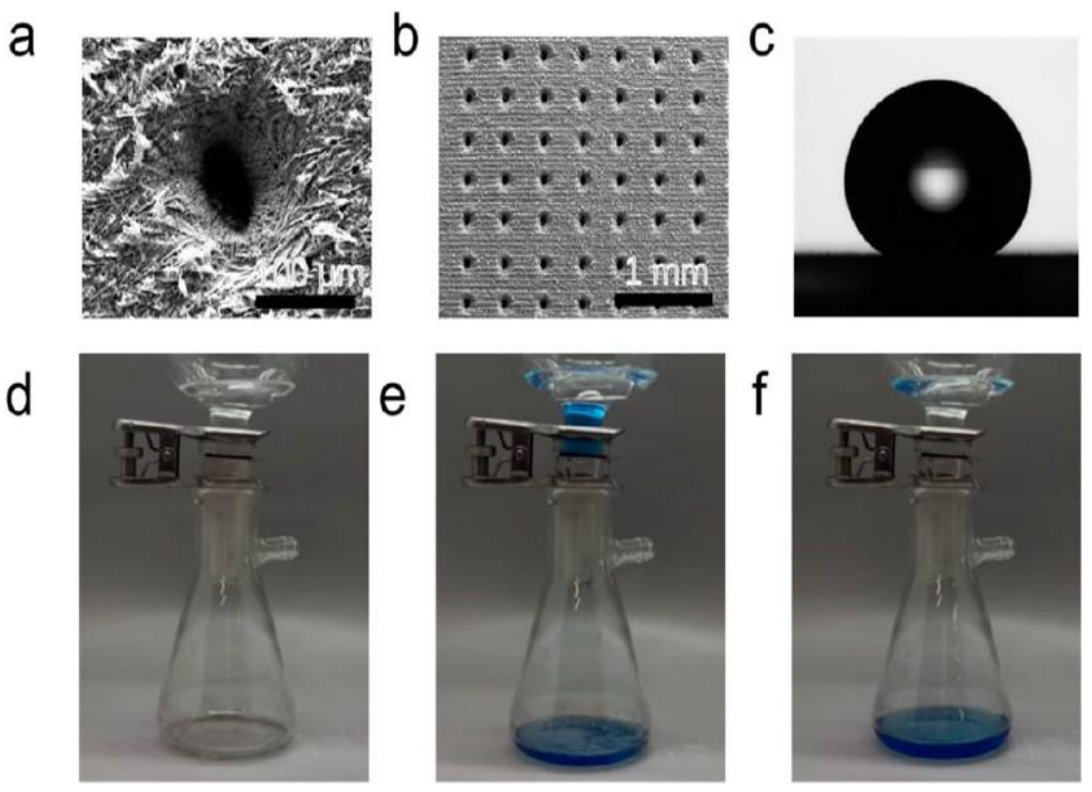

Figure 2.13 LIG as membrane for oil-water separation. (a,b) SEM image of LIG filter. (c) Water droplet on the surface of the LIG filter; the measured contact angle is $155^{\circ}$. (d-f) Filtration of $\mathrm{CHCl}_{3} / \mathrm{H}_{2} \mathrm{O}$ mixture with the LIG filter. (d) Water does not go through the filter; (e) $\mathrm{CHCl}_{3}$ goes through the filter; (f) water stays on top of the filter even after all of the $\mathrm{CHCl}_{3}$ went through [110]. 

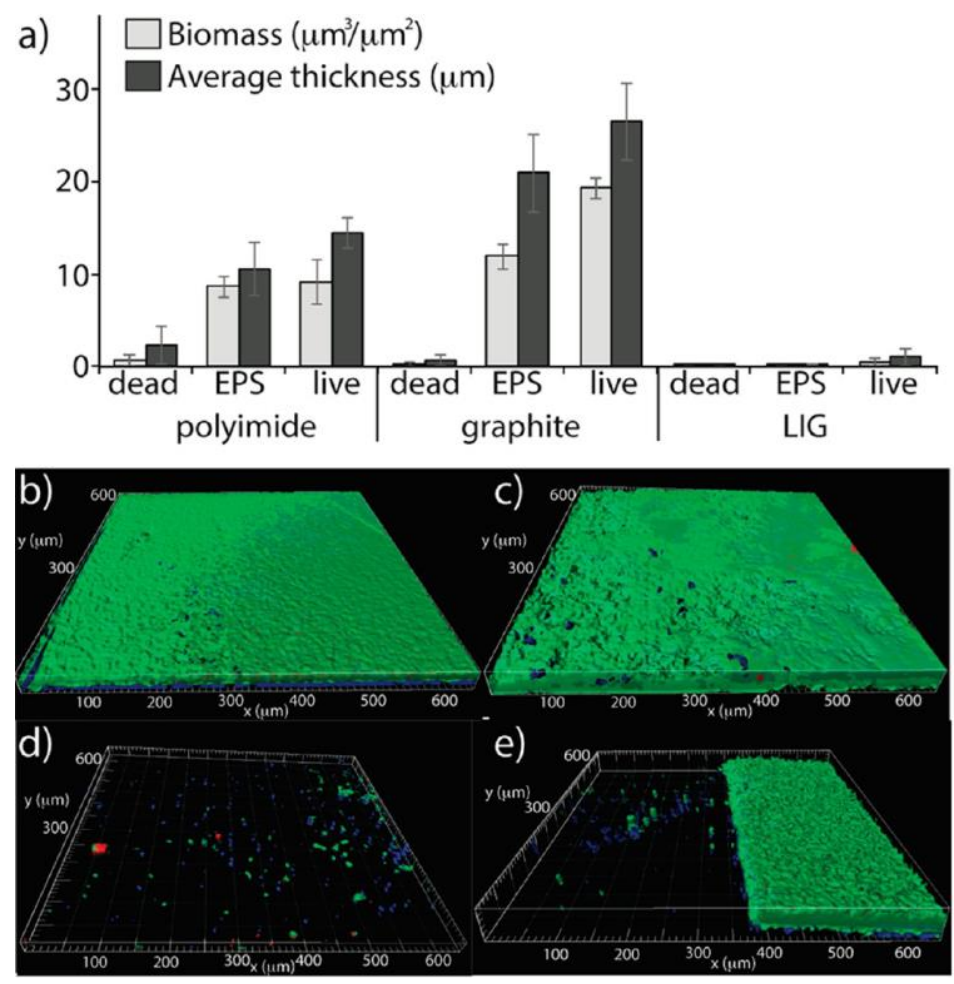

Figure 2.14 LIG film for microbial antifouling. (a) Biofilm growth on the PI, graphite, and LIG surfaces with P. aeruginosa showing biomass and average thickness. Representative IMARIS software images for (b) PI; (c) graphite; (d) LIG; (e) the interface between the LIG (left) and PI film (right). Green represents live bacteria, red represents dead bacteria, and blue represents EPS [23]. 


\title{
CHAPTER THREE
}

\section{Effect of Water Chemistry on The Aggregation and Photoluminescence Behavior of Carbon Dots}

\begin{abstract}
Carbon dots are rapidly emerging carbon-based nanomaterials that, due to their growing applications, will inevitably find their way to natural waters; however, their environmental fate is mostly unknown. Carbon dots with different surface functionality were fabricated and characterized by TEM and FT-IR. Their surface charge, given by the zeta potential, and their hydrodynamic diameter in suspension were investigated under a variety of environmentally relevant conditions. The effect of ionic strength was studied in the presence of monovalent $(\mathrm{NaCl})$ and divalent $\left(\mathrm{CaCl}_{2}\right)$ cations, for $\mathrm{pH}$ levels from 3 to 11; humic acid was used as a model for dissolved natural organic matter. Total potential energies of interactions were modeled by classical DLVO theory. The experimental results showed that water chemistry altered the surface charge of the nanomaterials, but their hydrodynamic size could not be correlated to those changes. Evidence of specific interactions was found for the amino functionalized particles in most cases, as well as the plain carbon dots in the presence of $\mathrm{Ca}^{2+}$ and humic acid. Nanoparticles remained largely stable in suspension, with some exception at the highest ionic strength considered. DLVO theory did not adequately capture the aggregation behavior of the system. Moreover, cation and/or humic acid adsorption negatively affected the emission intensity of the particles, suggesting limitations to their use in natural water sensing applications. The
\end{abstract}


particular stability shown by the carbon dots results in exposure to organisms in the water column and the possibility of contamination transported to significant distances from their source.

Keywords: Carbon dots; Photoluminescence; Aggregation

\subsection{Introduction}

Carbon dots (CDs) constitute a class of quasi-spherical, photoluminescence carbon nanoparticles with sizes below $10 \mathrm{~nm}$ [1]. Their easy fabrication and presumably negligible environmental impact make them an attractive alternative to inorganic quantum dots $[2,3]$. Early studies have shown CDs are biocompatible and less cytotoxic than Cd-based quantum dots and may even inhibit cancer cells [4-6]. These fluorescent nanoparticles have been successfully utilized in biomedicine, optoelectronics, catalysis, and chemical sensors [84]. Their application in the environmental field is particularly promising. Semiconductor quantum dots (SQDs) and graphene quantum dots (GQDs) can be distinguished within the large group of carbon-based nanoparticles since SQDs and GQDs are perfect spherical nanocrystals of metallic atoms and nanosheets of $\mathrm{sp}^{2}$ carbons, respectively, while the other types of carbon- dots have an undefined carbogenic structure, being in some situations a combination of crystalline structures resembling graphene layers combined with amorphous carbon. Carbon particles exhibiting a crystalline core based on a mixture of $\mathrm{sp}^{2}$ and $\mathrm{sp}^{3}$ carbons are termed carbon quantum dots (CQDs) while those composed of a mixture of primarily $\mathrm{sp}^{3}$ carbons in a disordered structure carbon nanodots 
(CNDs) [118].

The photoluminescence (PL) properties of carbon dots vary sensitively with size, and emissions are red-shifted with increasing size [84]. At present, the PL mechanism of carbon dots (CDs) is still under debate [119], although different researchers have attributed their emission properties to quantum confinement effect [120], surface states determined by hybridization of the carbon backbone and the connected chemical groups [121,122], fluorophores with different degrees of p-conjugation [82][96], and the recombination of electron-hole pairs localized within small $\mathrm{sp}^{2}$ carbon clusters embedded within a sp ${ }^{3}$ matrix [123]. Furthermore, the PL can be tuned via surface modification or via electron/energy transfer, and numerous capping strategies have been reported [84].

CDs characteristics can be tuned by the choice of precursors during synthesis, surface passivation and functionalization. Two types of synthesis methods, top-down and bottomup, have been reported [124]. The top-down methods involve cutting carbon resources, most commonly using concentrated oxidizing acid $\left(\mathrm{HNO}_{3}\right.$ or $\mathrm{H}_{2} \mathrm{SO}_{4} / \mathrm{HNO}_{3}$ mixture) [125]. The bottom-up synthesis methods consist of thermal carbonization and acid dehydration of suitable molecular precursors [126]; passivation and/or the functionalization may be required to create highly PL CDs with tunable fluorescent features [118]. Carbon dots possess numerous surface reactive groups, that offer the chance for surface modifications. Passivation can enhance the quantum yields (QYs) of the CDs, changing the PL emission and meeting the requirements of selected applications [119]. Different strategies have been used to functionalize carbon dots with different types of molecules; for instance, macromolecules, chelating ligands, specific oligonucleotides, and metal ion or metalmediated DNA binding for heavy metal detection $[118,127]$. Acid oxidative treatment, 
typically using $\mathrm{HNO}_{3}$, can introduce carbonyl functionalities at several carbon surfaces. These groups enhance the carbon dots water solubility, which is very important for biologically applications. Furthermore, the PL can be increased significantly by surface passivation agents, as for example, amino-terminated reagents [1].

As interest for both fundamental research and applications has surged dramatically in the last few years, it is inevitable CDs will find their way to natural water sources where, unlike the more well studied fullerenes and carbon nanotubes, their environmental impact and fate is mostly unknown. The presumed negligible environmental impact of carbon dots can only be confirmed if their behavior in natural systems is fully understood. However, the colloidal stability of CDs in environmentally relevant aqueous suspensions has not been investigated, and neither the effect of those conditions on their PL properties.

Due to their small size and large surface area, adsorption processes are expected to play a key role in determining the physicochemical properties of CDs [9]. Adsorption may alter the surface characteristics of the nanoparticles so that they become dispersed and thus, mobile in aqueous media [14]. Natural organic matter, e.g. humic acid (HA), and exopolymeric substances, e.g. proteins and polysaccharides, have been shown to adsorb onto carbon nanotubes and act as stabilization agents [13]; these macromolecules can potentially interact with CDs surfaces by $\pi-\pi$ bonding and hydrophobic effects, due to the presence of $\mathrm{C}=\mathrm{C}$ double bonds as well as aromatic rings [17].

CDs are well known for their colloidal stability in water suspensions at low ionic strength; their PL is stable under those conditions but generally expected to decline if aggregation occurs. Other matrix chemistry effects have been reported. For example, their florescence intensities were shown to be $\mathrm{pH}$-dependent, decreasing with increasing $\mathrm{pH}$ 
[128]. Furthermore, a study showed no significant change in fluorescence quenching of carbon dots by HA up to a concentration of $10 \mathrm{ppm}$ [118], while another study reported that the quenched fluorescence intensity of surface functionalized QDs was linearly proportional to the concentration of $\mathrm{Hg}^{2+}$ under optimum conditions [127].

In summary, there is extensive literature on inorganic nanoparticle stability in natural waters, as well as for some common carbon-based particles such as carbon nanotubes, fullerenes and a few studies on graphene oxides, but the aggregation and surface properties of carbon dots under environmental conditions have not been investigated. In view of the increasing amount of research devoted to carbon dots applications for sensing and imaging, an understanding of the colloidal stability is needed as a first step towards the prediction of environmental fate, transport and potential toxicity of these nanomaterials. It has been recognized that our knowledge on nanoparticle environmental behavior needs to be continuously revised and extended to account for new materials or novel commercial applications as they emerge, in order to accurately assess the associated risks [87].

The objective of this work is to investigate for the first time the colloidal stability of carbon dots, an emerging carbon nanomaterial, in natural water conditions (i.e. variable salinity, $\mathrm{pH}$, dissolved organic matter) and the effect of the transformations they undergo under such circumstances on their fluorescence properties. Two types of carbon dots were considered: as obtained from thermal treatment of organic molecules, and surface functionalized, to represent differences in their surface chemistry. The particles were synthesized and characterized with respect to size and surface chemistry. Aggregation was investigated under environmental relevant conditions and DLVO theory was applied to the modeling of particle stability. 


\subsection{Materials and methods}

\subsubsection{Synthesis}

Unfunctionalized or plain carbon dots (CDs) were synthesized following a modification of a previously published method (Wang et al., 2011). A 70\% (W/W) glycerol solution (Sigma, >99.5\%) was mixed with a $20 \%$ (W/W) solution of $\mathrm{NaH}_{2} \mathrm{PO}_{4}($ Fisher Scientific) in a 10:1 volume ratio. The solution was heated for $20 \mathrm{~min}$ at $900 \mathrm{~W}$ power in a domestic microwave. The product was allowed to cool to room temperature and the CDs resuspended by addition of water and continuous stirring until a homogenous brown colored suspension was obtained.

Amine capped CDs (a-CDs) were fabricated following a procedure described by Zheng el al. [129]. Briefly, $2.10 \mathrm{~g}$ of a $10 \mathrm{mmol}$ solution of citric acid (Sigma-Aldrich, ACS reagent, $>=99.5 \%$ ) were added to $10 \mathrm{~mL}$ of branched polyethylenimine ethylenediamine (BPEI) (Sigma-Aldrich) with an average molecular weight of $800 \mathrm{Da}$, as reported by the manufacturer. The reaction was heated to $170{ }^{\circ} \mathrm{C}$ in a silicone oil bath (Fisher Scientific) for $2 \mathrm{hr}$. The yellow colored products were naturally cooled to room temperature followed by addition of deionized water. The resulting solutions of both CDs and a-CDs were dialyzed with a Biotech CE dialysis membrane (Spectrum Labs, MWCO 1.0 kDa) against ultrapure water (specific resistance $18 \mathrm{M} \Omega^{*} \mathrm{~cm}$ ) for 2 days for purification. Water was replaced every 6 hr. Finally, CDs and a-CDs were freeze-dried. 


\subsubsection{Characterization}

The surface functional groups of the synthesized materials were investigated by Fourier-Transform Infrared Spectroscopy (FT-IR) in a Nicolet 4700 FT-IR spectrophotometer (Thermoscientific) and the spectra collected over a wavenumber range from 400 to $4000 \mathrm{~cm}^{-1}$. CDs and a-CDs were imaged by Transmission Electron Microscopy (TEM) in a Tecnai F30 Twin transmission electron microscope (FEI). TEM images were then analyzed using the software ImageJ to obtain the particle size distribution (Schneider et al., 2012). In brief, the TEM images were selected and imported to ImageJ, then the particles on the images were segmented by adjusting the threshold value and sizes were analyzed by the software. At least 400 particles were measured for each type of nanoparticle.

Hydrodynamic diameter $\left(D_{\mathrm{h}}\right)$ and zeta potential were measured with a ZetaSizer Nano ZS (Malvern Instruments, Worcestershire, U.K.), using a monochromatic coherent $\mathrm{He}-\mathrm{Ne}$ laser with a fixed wavelength of $633 \mathrm{~nm}$. Laser Doppler velocimetry was applied to characterize the electrophoretic mobility (EPM) of the nanomaterials in the various electrolyte solutions. Measured EPMs were converted to zeta-potential using the Smoluchowski equation (Elimelech et al., 1995).

$$
\mathrm{U}=\frac{\varepsilon \zeta}{\mu}
$$

where $U$ is the electrophoretic mobility, $\varepsilon$ is the dielectric constant of the solution, $\mu$ is its viscosity, and $\zeta$ is the zeta potential. Disposable folded capillary cells were employed.

The hydrodynamic properties of carbon dots were measured under a wide range of solution compositions, including monovalent and divalent electrolytes, represented by 
$\mathrm{NaCl}$ (reagent grade, Acros Organics) and $\mathrm{CaCl}_{2}$ (reagent grade, J.T. Baker) respectively. Humic acid (technical grade, Acros Organics) was introduced as a model of natural dissolved organic matter (NOM). The $\mathrm{pH}$ of the solution was adjusted by adding $\mathrm{HCl}$, (10 $\mathrm{mmol} / \mathrm{L}$, Fisher Scientific) or $\mathrm{NaOH}$ (10 mmol/L, Fisher Scientific) solutions.

UV-vis adsorption spectra for $\mathrm{CDs}$ and a-CDs were recorded using UV-vis spectrophotometer (Lab Tech, UV 8100B, USA) with scanning range 200-600 nm. Photoluminescence was measured on a Hitachi F-4500 Fluorescence Spectrophotometer, equipped with a $150 \mathrm{~W}$ Xenon lamp. Emission scans were performed at an excitation wavelength of $350 \mathrm{~nm}$. The slit width was $5 \mathrm{~nm}$ and scans were conducted in an emission wavelength range from $300 \mathrm{~nm}$ to $700 \mathrm{~nm}$ at a speed of $1,200 \mathrm{~nm} / \mathrm{min}$. Concentration of aCDs were kept constant at $200 \mathrm{mg} / \mathrm{L}$ for all absorption and fluorescence experiments; CDs concentrations were $1,600 \mathrm{mg} / \mathrm{L}$ for adsorption and 52,000 $\mathrm{mg} / \mathrm{L}$ for fluorescence experiments.

\subsubsection{DLVO Modeling}

The total interaction energy between particles as they approach each other ( $\left.V_{\mathrm{TOTAL}}\right)$ was calculated to determine the aggregation process, as described by the traditional DLVO theory. According to this theory, the stability of colloidal particles in aqueous environments is determined by the net effect of van der Waals interactions $\left(V_{\mathrm{VdW}}\right)$ and electrical double layer interactions $\left(V_{\mathrm{EDL}}\right)$ :

$$
V_{\text {Total }}=\mathrm{V}_{E D L}+\mathrm{V}_{v d w}
$$

Nanomaterials were modeled as spherical particles with diameter equal to their hydrodynamic diameter. Since dispersion forces are electromagnetic in character, they are 
subject to a retardation effect [1,9]. Thus, van der Waals interactions were calculated with retardation [130,131] by Equation (3.3):

$$
V_{v d w}=-\frac{A_{i w i} a}{12 h}\left[\left(1+\frac{14 h}{\lambda}\right)\right]^{-1}
$$

where $a(\mathrm{~nm})$ is the particle radius; $h(\mathrm{~nm})$ is the surface-to-surface distance between two particles; $A_{\text {iwi }}$ is the Hamaker constant, $6.26 \times 10^{-21} \mathrm{~J}$ was employed in this study [132], $\lambda$ is the characteristic wavelength of the dielectric and usually assumed to be $100 \mathrm{~nm}$. Electrostatic double layer interactions were calculated using the following Equations (3.4) $-(3.6)$ :

$$
\begin{gathered}
V_{E D L}=\frac{64 \pi a_{P} n_{\infty}}{K^{2}} k T \gamma^{2}[\exp (-K h)] \\
n_{\infty}=1000 \times N_{A} \times C_{s} \\
\gamma=\operatorname{Tanh}\left(\frac{Z e \psi}{4 k T}\right)
\end{gathered}
$$

where $a_{\mathrm{P}}(\mathrm{nm})$ is the radius; $h(\mathrm{~nm})$ is the surface-to-surface distance between two particles; $n_{\infty}$ is bulk number density of ions which is calculated according to Equation (3.5) where $C_{\mathrm{S}}\left(\mathrm{mol} / \mathrm{dm}^{3}\right)$ is the electrolyte molar concentration, and $N_{A}($ atoms $/ \mathrm{mol})$ is Avogadro's constant; $k(\mathrm{~J} / \mathrm{K})$ is Boltzmann constant; and $T(\mathrm{~K})$ is absolute temperature.

The parameter $\gamma$ is calculated according to Equation (3.6), where $Z$ is the charge of the ions; $\psi(\mathrm{V})$ is the experimental zeta potential; $e$ (Coulombs) is the electron charge; and $K\left(\mathrm{~m}^{-1}\right)$ is the Debye length as calculated below:

$$
K=2.32 \times 10^{9} \sqrt{\sum C_{i} z_{i}^{2}}
$$


where $C_{\mathrm{i}}\left(\mathrm{mol} / \mathrm{m}^{3}\right)$ is ion concentration; $z_{i}$ the valence of ion $i$ including sign of charge. The application of Equation (3.5) is limited to for $h<\left\langle a_{P}\right.$ and $\left.K a_{P}\right\rangle 5$.

\subsection{Results and discussion}

The size, morphology and structure of the nanoparticles were investigated by TEM; the corresponding images of a-CDs and CDs are shown in Figure 3.1 (a) and (c). The image analysis (Figure 3.1 (b) and (d)) reveled an average particles size of $5.1 \mathrm{~nm}$ for a-CDs and $1.8 \mathrm{~nm}$ for CDs with size ranges between 1 to $9 \mathrm{~nm}$ and 1 to $3 \mathrm{~nm}$ respectively, which is in good agreement with previous reports in the literature [86]. Figure 3.2 (a) and (b) represent HRTEM images for CDs and a-CDs respectively. The images reveal the lattice spacing of $0.32 \mathrm{~nm}$ for both type of carbon dots, which agree with (002) spacing of graphitic carbon [133].

The surface chemistry was investigated by FT-IR (Figure 3.3). The FT-IR spectrum of CDs (Figure 3.3 (b)) showed the existence $\mathrm{O}-\mathrm{H}, \mathrm{C}=\mathrm{C}$, epoxy groups at the positions of 3223, 1450 and 1058, respectively. In contrast, inspection of the a-CDs spectrum suggests abundant presence of amino groups on the surface (Figure 3.3 (a)). The stretching vibrations of N-H at $3286 \mathrm{~cm}^{-1}, \mathrm{C}-\mathrm{H}$ at $2958 \mathrm{~cm}^{-1}$ and $2819 \mathrm{~cm}^{-1}$ were observed in the high wavenumber region. Two strong peaks at $1658 \mathrm{~cm}^{-1}$ and $1570 \mathrm{~cm}^{-1}$ contributed to the amide linkage formed. The adsorption bands of $\mathrm{C}=\mathrm{C}, \mathrm{C}=\mathrm{N}, \mathrm{C}=\mathrm{C}-\mathrm{O}$ and epoxy groups were identified at 1466, 1362, 1300, $1111 \mathrm{~cm}^{-1}$, respectively 

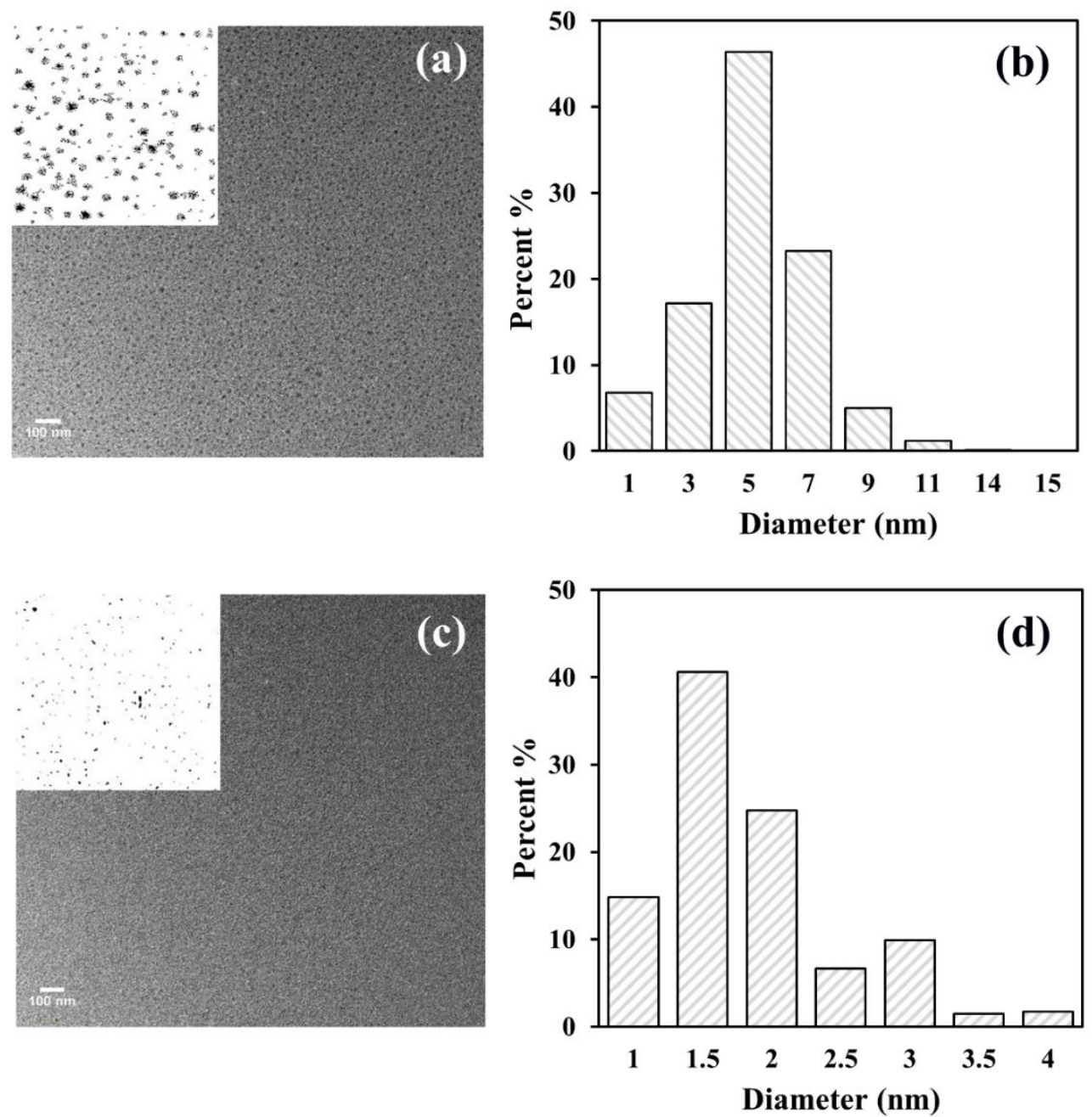

Figure 3.1 (a), (c) Representative transmission electron microscopy image of amine capped carbon dots and plain carbon dots: insets represent the segmented particles after subtracting the background using ImageJ software. (b), (d) Size distribution of a-CDs and CDs, as percent number of particles. 

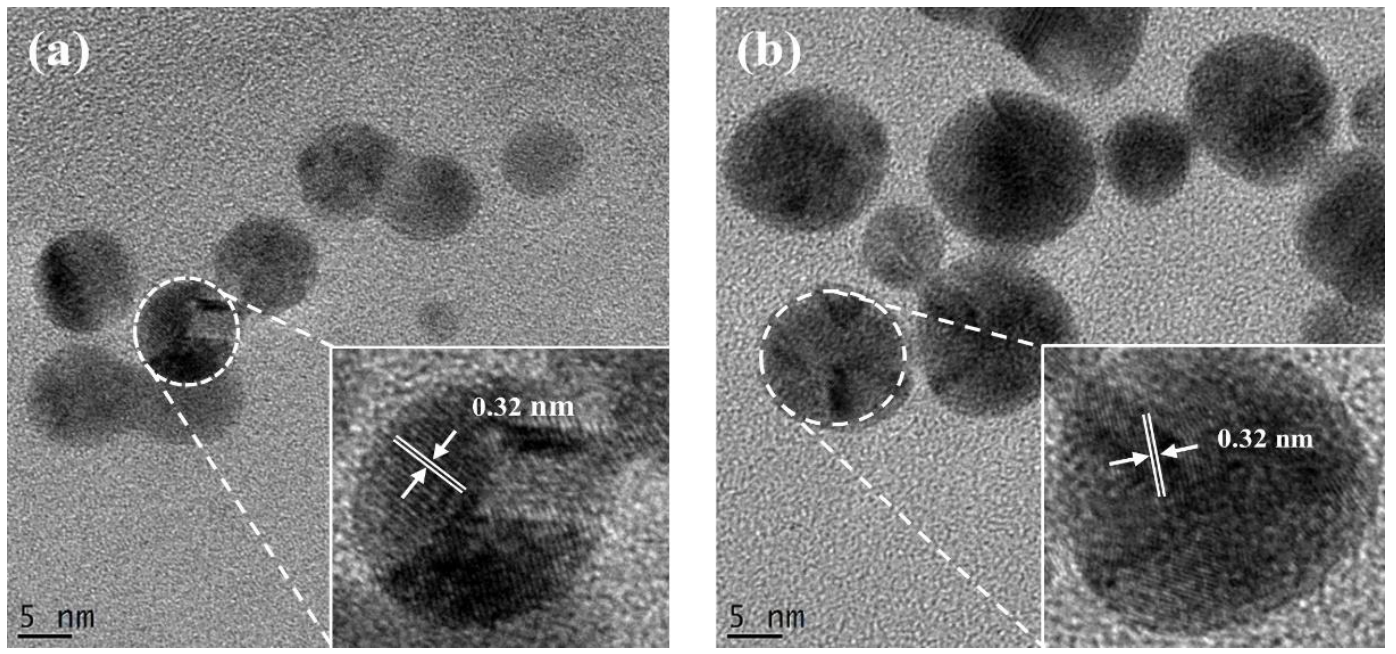

Figure 3.2 (a), (b) Representative high-resolution transmission electron microscopy images of aCDs and CDs respectively with lattice parameters of $0.32 \mathrm{~nm}$.
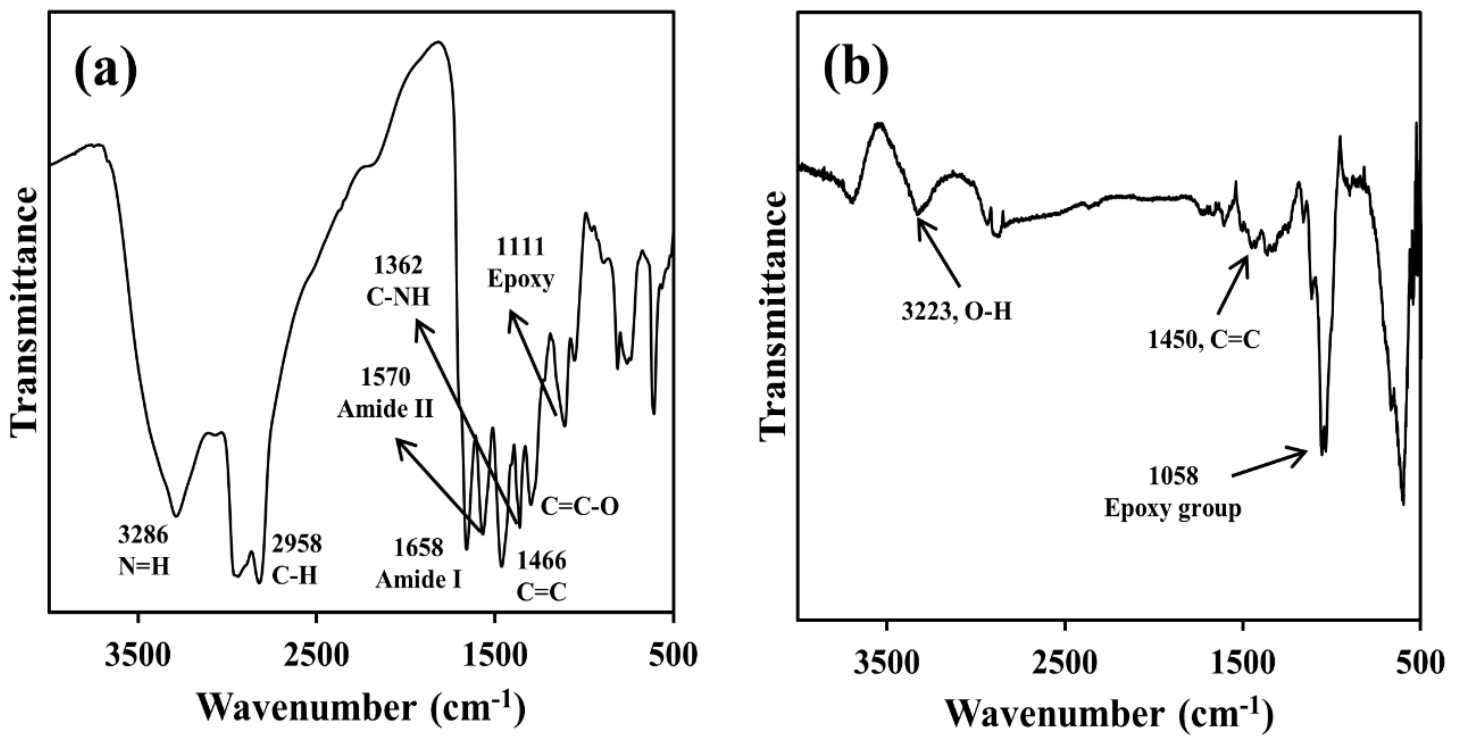

Figure 3.3 Fourier transform infrared spectroscopy spectra of: (a) a-CDs. (b) CDs

As prepared, both nanoparticles formed stable colored suspensions in ultrapure water due to their highly hydrophilic nature. The resulting $\mathrm{pH}$ was 5.7 and 9.8 for the CDs and a-CDs suspensions, respectively. This result indicates that the basic functional groups were 
exposed on the surface of the nanoparticles. Under these conditions, their hydrodynamic diameter was determined to be $1.1 \pm 0.4 \mathrm{~nm}(\mathrm{CDs})$ and $1.9 \pm 0.1 \mathrm{~nm}(\mathrm{a}-\mathrm{CDs})$ and their surface charge, given by the zeta potential, was $-11.4 \pm 3.5 \mathrm{mV}$ (CDs) and $6.9 \pm 0.6 \mathrm{mV}$ (a-CDs). It is important to note that the mean particle size measured by dynamic light scattering (DLS) in solution was smaller than the TEM based determination. DLS size measurements are usually expected to yield larger values than those from microscopy images, given that they represent hydrodynamic diameters, which led to the conclusion that there was some degree of aggregation during the drying stage in the preparation of TEM samples, even though extreme care was exercised to avoid it with highly diluted particle suspensions and different evaporation methods. As sample drops were deposited on the carbon mesh, carbon dots possibly accumulated in one spot, producing an image compatible with larger particle size. The combined effect of small particle size and surface charge was responsible for the easy dispersion and remarkable stability of carbon dots suspensions. Moreover, suspensions prepared in the laboratory remained free of sediments for as long as 2 months.

\subsubsection{Influence of ionic strength}

Figure 3.4 summarizes the effect of ionic strength (IS) and electrolyte type $(\mathrm{NaCl}$, $\mathrm{CaCl}_{2}$ ) on zeta potential of $\mathrm{CDs}$ and a-CDs. $\mathrm{CDs}$ were negatively charged for all $\mathrm{NaCl}$ and $\mathrm{CaCl}_{2}$ concentrations considered; in contrast, a-CDs exhibited positively charged surfaces. Figure 3.4 (a) shows that zeta potential values of CDs became less negative from $-11.4 \pm$ 3.5 to $-3.9 \pm 0.5 \mathrm{mV}$, as IS of the $\mathrm{NaCl}$ solution increased from 1 to $100 \mathrm{mmol} / \mathrm{L}$. This less negative surface charge can be attributed to the electrical double layer compression, as 
predicted by classical colloidal theory $[134,135]$. As IS increases and the thickness of the electrical double layer diminishes, a faster decay of the potential energy of interaction with distance from the surface occurs, placing the shear plane, where zeta potential is measured, at a location of lower absolute potential value. In contrast, IS variation caused a moderate zeta potential increase for the already positively charged a-CDs, followed by a decrease at higher electrolyte concentrations. The zeta potential values of a-CDs increased from $5.4 \pm$ 0.6 to $15.4 \pm 0.6 \mathrm{mV}$, as $\mathrm{IS}(\mathrm{NaCl})$ increased from 1 to $10 \mathrm{mmol} / \mathrm{L}$ and decreased to $8.64 \pm$ $0.6 \mathrm{mV}$ at $50 \mathrm{mmol} / \mathrm{L}$ level. The effect was more noticeable for $\mathrm{Na}^{+}$(monovalent) than $\mathrm{Ca}^{2+}$ (divalent) cations. This observation suggests the occurrence of specific interactions between the dissolved electrolytes and the nanoparticles, that gave rise to an apparent higher surface charge; however, this phenomenon was overcome by the double layer compression at higher ionic strength conditions, leading to a reduced magnitude of the zeta potential.
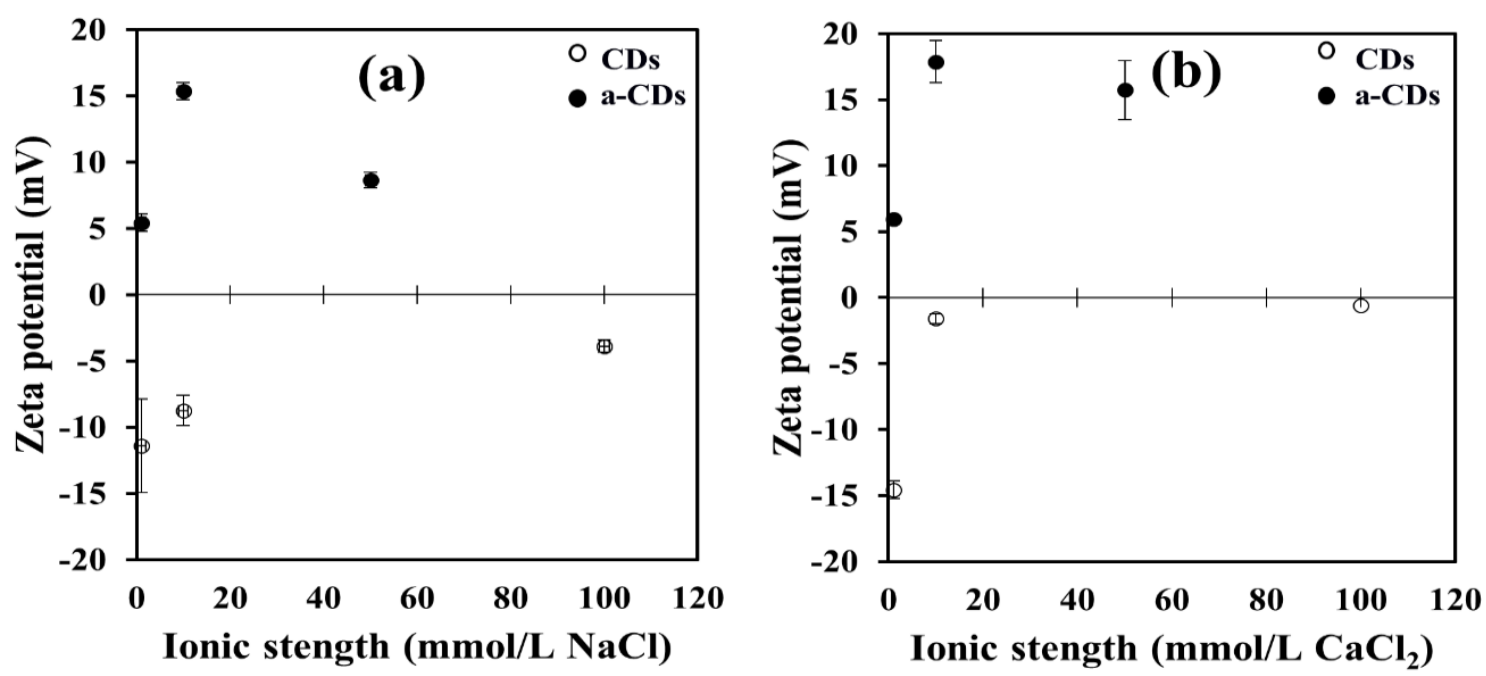

Figure 3.4 Zeta potential of CDs $(\mathrm{pH}=5.7)$ and a-CDs $(\mathrm{pH}=9.7)$ as a function of ionic strength $\left(\mathrm{NaCl}\right.$ and $\left.\mathrm{CaCl}_{2}\right)$. 
Figure 3.5 (a) shows a significant increase of CDs hydrophilic diameter $\left(D_{\mathrm{h}}\right)$ from 1.1 \pm 0.5 to $533.2 \pm 23.1 \mathrm{~nm}$ suspended in $\mathrm{NaCl}$ solutions. The high $D_{\mathrm{h}}$ at $100 \mathrm{mmol} / \mathrm{L}$ is in coincidence with the low absolute zeta potential value. The presence of $\mathrm{NaCl}$ did not affect the $D_{\mathrm{h}}$ of a-CDs appreciably as shown in Figure 3.5 (b) and the $D_{\mathrm{h}}$ remained approximately constant around $2 \mathrm{~nm}$. In most natural freshwater bodies, ionic strength is below $10 \mathrm{mmol} / \mathrm{L}$ [9], which indicates that aggregation is not expected for CDs in those aquatic environments; however, seawater and groundwater conditions may lead to substantial size increase.

The zeta potential and hydrodynamic characterization of CDs, and a-CDs in the presence of a divalent cation $\left(\mathrm{Ca}^{2+}\right)$ are shown in Figure $3.4(\mathrm{~b})$ and $3.5(\mathrm{~b})$, respectively. $\mathrm{Ca}^{2+}$ influenced both zeta potential and hydrodynamic diameter more noticeable than the monovalent $\mathrm{Na}^{+}$. Figure 3.4 (b) shows that zeta potential values of CDs are significantly altered by $\mathrm{Ca}^{2+}$, as they became less negative from $-14.8 \pm 0.7$ to $-0.6 \pm 0.04 \mathrm{mV}$, as IS $\left(\mathrm{CaCl}_{2}\right)$ increased from 1 to $100 \mathrm{mmol} / \mathrm{L}$. The almost null zeta potential observed may be attributed to specific adsorption of $\mathrm{Ca}^{2+}$ on the negatively charged surface groups of the CDs. On the other hand, the zeta potential of a-CDs increased from $5.9 \pm 0.3$ to $17.9 \pm 1.6$ $\mathrm{mV}$, as the IS $\left(\mathrm{CaCl}_{2}\right)$ increased from 1 to $10 \mathrm{mmol} / \mathrm{L}$ but remained virtually constant from 10 to $50 \mathrm{mmol} / \mathrm{L}$.

Figure 3.5 (b) shows the $D_{\mathrm{h}}$ of CDs increased to $19.3 \pm 1.0,20.8 \pm 1.7$, and $8.6 \pm 5.3$ $\mathrm{nm}$ in presence of 1,10 , and $100 \mathrm{mmol} / \mathrm{L}$ IS given by $\mathrm{CaCl}_{2}$ respectively. The stability behavior does not appear to be affected by the change in surface charge of the nanoparticles under those conditions. In comparison to the small diameters of the nanoparticles, hydrated $\mathrm{Ca}^{2+}$ ions have a non-negligible size of $1.2 \mathrm{~nm}[136]$, the specific adsorption on CDs may 
increase the size of nanoparticles and cause moderate aggregation among them at low concentrations, while tend to repulse each other at higher concentrations. Similar to $\mathrm{Na}^{+}$, no effects of $\mathrm{Ca}^{2+}$ on a-CDs were observed, and $D_{\mathrm{h}}$ remained constant around $3 \mathrm{~nm}$. The observed behavior of the BPEI coated a-CDs were strikingly different from CDs under these conditions, probably due to radical differences in surface chemistry given by the branched amino groups on the surface. a-CDs exhibit a very good stability in the presence of dissolved salts for all the conditions investigated.
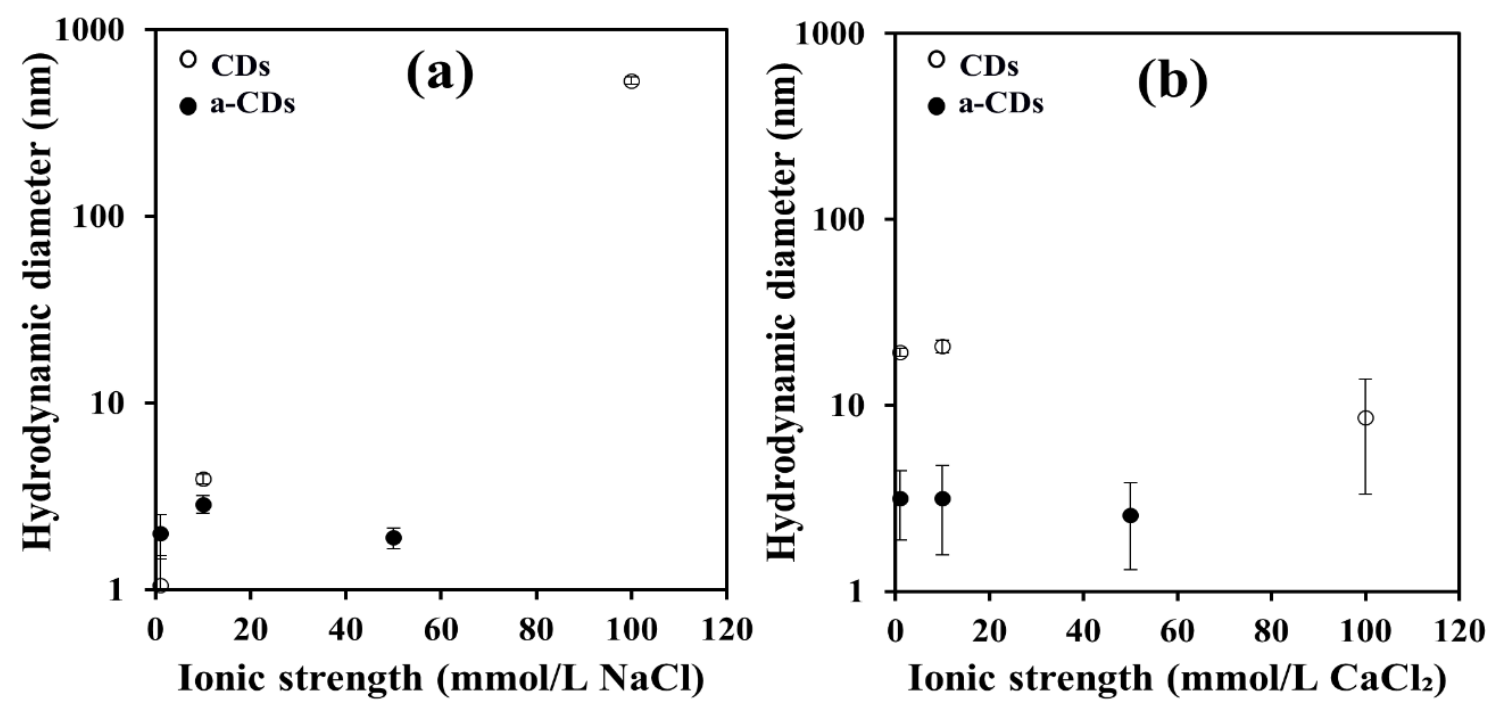

Figure 3.5 Hydrodynamic diameter of $\mathrm{CDs}(\mathrm{pH}=5.7)$, a-CDs $(\mathrm{pH}=9.7)$ as a function of ionic strength $\left(\mathrm{NaCl}\right.$ and $\left.\mathrm{CaCl}_{2}\right)$

\subsubsection{Influence of $\mathrm{pH}$}

Our measurements indicated that zeta potential values of $\mathrm{CDs}$ were $\mathrm{pH}$ sensitive and they decreased with increasing $\mathrm{pH}$. Zeta potential of CDs remained negatively charged over a $\mathrm{pH}$ range from 2 to 11 in the presence of $\mathrm{NaCl}$ and $\mathrm{CaCl}_{2}$, and positively charged 
over the $\mathrm{pH}$ range from 2 to 10 for a-CDs (Figure 3.6). In the presence of $1 \mathrm{mmol} / \mathrm{L}$ IS $(\mathrm{NaCl}), \mathrm{CDs}$ ' zeta potential decreased from $-1.4 \pm 0.7$ to $-35.1 \pm 2.2 \mathrm{mV}$, as $\mathrm{pH}$ increased from 3 to 11 respectively. The zeta potential decline can be related to the deprotonation of surface functional group, such as carboxyl and hydroxyl groups. Wang showed that CDs also contain hydroxyl and carboxyl functional groups, which agrees with our FTIR results [86]. DLS results (Figure 3.7 (a)) show that the $\mathrm{pH}$ did not have a noticeable effect on CDs stability from $\mathrm{pH} 4$ to $11 \mathrm{in}$ presence of $\mathrm{NaCl}$. The $D_{\mathrm{h}}$ of CDs was around $1 \mathrm{~nm}$, and then increased only slightly as $\mathrm{pH}$ decreased from 4 to 2 .

When CDs were suspended with a background ionic strength of $1 \mathrm{mmol} / \mathrm{L}$ given by $\mathrm{CaCl}_{2}$, the zeta potential decreased from $-3.3 \pm 0.3$ to $-20.1 \pm 4.1 \mathrm{mV}$ as a $\mathrm{pH}$ increased from 3 to 11 (Figure 3.6 (b)). The CDs were mostly well dispersed at $\mathrm{pH} 9$, as evidenced by a measured $D_{\mathrm{h}}$ of $1 \mathrm{~nm}$, but showed slight aggregation for other $\mathrm{pHs}$ considered, when the $D_{\mathrm{h}}$ was higher but under $40 \mathrm{~nm}$.
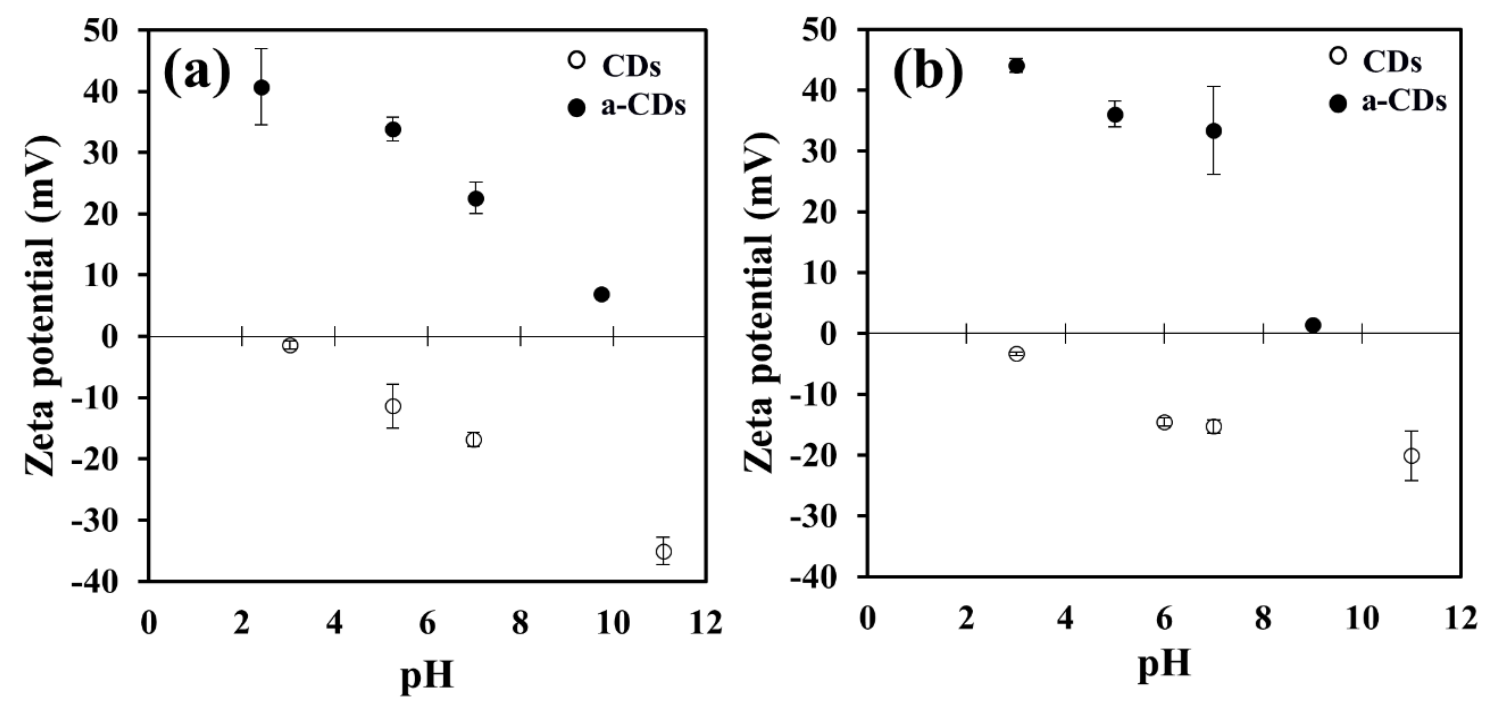

Figure 3.6 Zeta potential of CDs, a-CDs as a function of $\mathrm{pH}$. (a) $1 \mathrm{mmol} / \mathrm{L}$ ionic strength given by $\mathrm{NaCl}$. (b) $1 \mathrm{mmol} / \mathrm{L}$ ionic strength given by $\mathrm{CaCl}_{2}$. 
a-CDs zeta potentials decreased from $+40.73 \pm 6.2$ to $+6.88 \pm 0.6 \mathrm{mV}$ in presence of 1 $\mathrm{mmol} / \mathrm{L} \mathrm{NaCl}$ as $\mathrm{pH}$ increased from 2 to 10 (Figure 3.6 (a)); on the other hand, the $D_{\mathrm{h}}$ showed little to no variation (Figure 3.7 (a)). The a-CDs suspensions in $1 \mathrm{mM}$ ionic strength $\mathrm{CaCl}_{2}$ showed a decreasing zeta potential from 44.1 to $1.4 \mathrm{mV}$ when $\mathrm{pH}$ increased from 3 to 9 and remained almost constant at higher $\mathrm{pH}$ values. The presence of amino groups indicated that $\mathrm{pH}$ significantly changed the state of charge and the properties of CDs. Amino groups have a $\mathrm{p} K_{\mathrm{a}}$ value that usually varies between 9 and 10 [86,137]. The basic groups resulted in a higher positively charged surface due to protonation of amino groups for all $\mathrm{pH}$ range investigated. The surface charge increased with decreasing $\mathrm{pH}$, as a result of further protonation of the surface. The measured $D_{\mathrm{h}}$ was small at pHs between 7 to 9 , an indication of stability, but increased at lower $\mathrm{pHs}$, where nano sized aggregates were formed, mostly below $30 \mathrm{~nm}$. As discussed, divalent cations have greater influence on the aggregation of negatively charge nanomaterials compared to monovalent ions [138]. In this situation, $\mathrm{pH}$ did not appear to be a key factor affecting hydrodynamic size, but it significantly changed the surface charge due to protonation or deprotonation of surface functional groups on CDs and a-CDs [139]. Moreover, size and surface charge values for both particles in ultrapure water suspensions were found to be similar to those under 1 $\mathrm{mmol} / \mathrm{L} \mathrm{NaCl}$, within the experimental error, for the $\mathrm{pH}$ range between 2-11 (data not shown). Given the potential inaccuracy of $\mathrm{pH}$ determinations for low ionic strength solutions, i.e. ultrapure water, the $1 \mathrm{mmol} / \mathrm{L} \mathrm{NaCl}$ condition was used as the indifferent electrolyte condition for comparisons in this study [140]. 

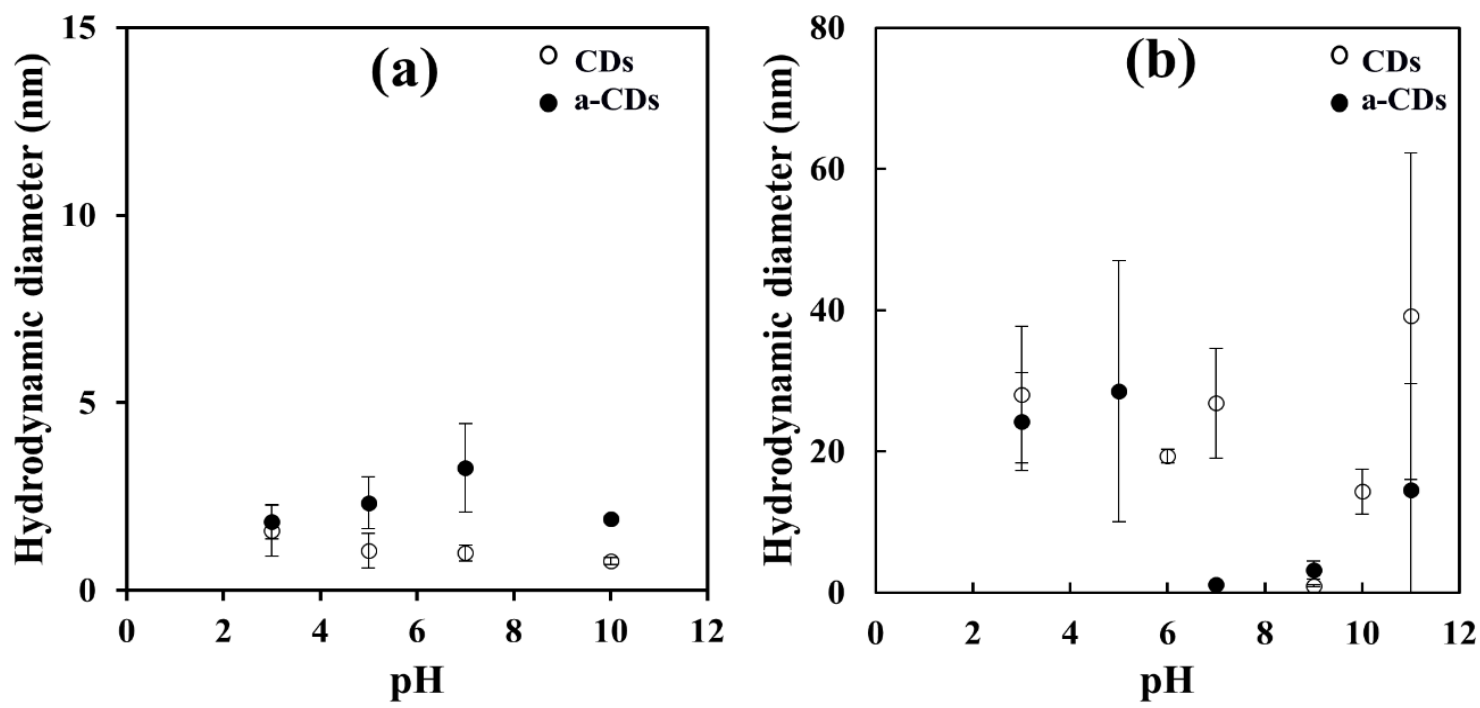

Figure 3.7 Hydrodynamic diameter of a-CDs, CDs as a function of $\mathrm{pH}$. (a) $1 \mathrm{mmol} / \mathrm{L}$ ionic strength given by $\mathrm{NaCl}$. (b) $1 \mathrm{mmol} / \mathrm{L}$ ionic strength given by $\mathrm{CaCl}_{2}$.

\subsubsection{Influence of natural organic matter}

The influence of dissolved natural organic matter on the stability of CDs and a-CDs was investigated in the absence of added electrolytes at circumneutral $\mathrm{pH}$. NOM has been shown to stabilize nanoparticles by inhibiting the formation of aggregates by adsorption on the surface of the nanomaterials, imparting a negative charge, and through steric effects, preventing particles to approach each other to close distances at which Van der Waals interaction become significant to overcome repulsion forces. Whether NOM results in stabilization or destabilization depends on several factors, including chemical nature of the organic molecules, concentration, and solution chemistry. Stabilization usually results from NOM forming a charged layer outside the particle, while destabilization results from particles being bridged by larger NOM molecules and/ or $\mathrm{Ca}^{2+}$ which dominate interactions between nanoparticles [138]. 
The $D_{\mathrm{h}}$ of CDs was increased from $1.1 \mathrm{~nm}$ to $50-200 \mathrm{~nm}$ range in presence of humic acid (as shown in Figure 3.8 (a)); the $D_{\mathrm{h}}$ of a-CDs was also increased with existing NOM and reached $491 \mathrm{~nm}$ at the concentration of $50 \mathrm{mg} / \mathrm{L} \mathrm{HA}$. Surprisingly, particle sizes decreased at higher HA concentrations. This phenomenon, observed for both CDs and aCDs, can be explained by the initial bridging effect at relatively low concentration when a single HA molecule may interact with multiple nanoparticles. At increasing HA concentrations, HA molecules are present is excess and competition for surface sites resulted in HA molecules being associated with individual nanoparticle surfaces. Results are also compatible with higher adsorption affinity for a-CDs, which had a more noticeable size change. The functionalized a-CDs have abundant amine groups on the surface, which may form complexes with HA [80]. Some studies have utilized this property to improve the performance of HA absorbents by functionalizing with polyethylenimine (PEI) $[141,142]$. The absorption of HA on a-CDs may result in agglomeration and produce the size growth.

Zeta potential of CDs and a-CDs as a function of HA concentration are shown in Figure 3.8 (b). CDs zeta potential decreased from $-16.8 \pm 1.1 \mathrm{mV}$ to as low as $-58 \pm 2.7 \mathrm{mV}$, at a concentration of $50 \mathrm{mg} / \mathrm{L} \mathrm{HA}$. At neutral $\mathrm{pH}$ values, the anionic HA was adsorbed onto the surface, yielding a rather low negative zeta potential. However, a-CDs zeta potential was positive and even higher with existence of $\mathrm{HA}$, reaching values above $45 \mathrm{mV}$ for all concentrations. Zhang and Bai [143] studied HA adsorption on chitosan-coated granules by X-ray photoelectron spectroscope and showed the formation of $-\mathrm{NH}_{3}{ }^{+} \ldots-\mathrm{OOC}-\mathrm{R}$ or $\mathrm{NH}_{3}{ }^{+} \ldots-\mathrm{O}-\mathrm{C}_{6} \mathrm{H}_{4}-\mathrm{R}$ surface complexes, which involved the generation of $-\mathrm{NH}_{3}{ }^{+}$as a result of interaction between the amino groups and the absorbed HA. We postulate that this HA 
induced protonation of the amino groups may be the reason for the increased positive charge observed in our experiments for a-CDs in the presence of HA.
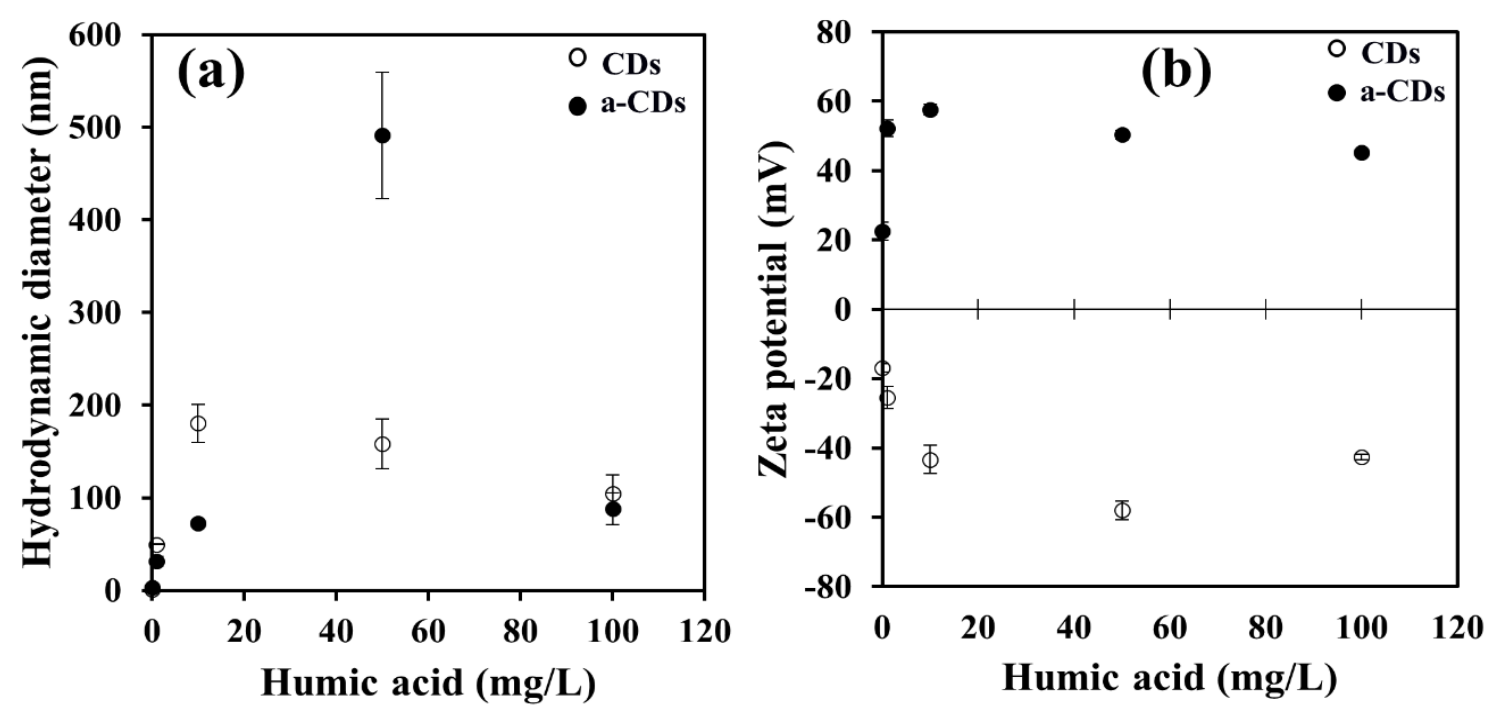

Figure 3.8 Hydrodynamic diameter (a) and zeta potential (b) of CDs, a-CDs suspended in humic acid solutions of variable concentration at neutral $\mathrm{pH}$.

\subsubsection{DLVO modeling}

DLVO theory was used to model CDs and a-CDs behavior. The ratio of total interaction energies to thermal energy $k T$, was calculated as a function of separation distance and plotted for $\mathrm{pH}$ ranging from 3 to10, and IS levels of 1, 10 and $100 \mathrm{mmol} / \mathrm{L}$ for CDs (Figure 3.9) and a-CDs (Figure 3.10). Both CDs and a-CDs exhibited very low interaction energies for all ionic strength and $\mathrm{pH}$ conditions, with values in the same order of magnitude as the thermal energy of the system. Such a system is expected to be unstable; the extremely weak repulsion forces present will allow particles to approach to very close separation distances, where attraction predominates. Thus, particles may aggregate irreversibly in the deep primary energy minimum. 
Pelley and Tufenkji [144] demonstrated that particle interaction energies are very sensitive to particle size and changes. The height of repulsive energy barrier and the depth of secondary energy well increased dramatically with increasing particle diameter. The small particle size of CDs had contributed to the low calculated energies barrier, leading to instability predictions for most of the modeled conditions. However, experiments revealed the remarkable stability of carbon dots, except for the highest ionic strength level conditions. The modeling equations contemplate two effects of the addition of salts on repulsion: a decrease in zeta potential and an increase in Debye length $(K)$; both will decrease the repulsion at a given separation distance, and hence promote aggregation. Even if relatively accurate predictions were obtained for some conditions (i.e. CDs suspended in $\mathrm{NaCl}$, highest ionic strength conditions), the DLVO modeling was largely in disagreement with experimental results, especially for a-CDs.

Classic DLVO relies exclusively in the interplay of electrostatic and Van der Waals interactions to describe colloidal stability. However, our experimental work evidenced a lack of correspondence between surface charge and size variations, which suggests a negligible influence of electrostatic repulsion in the colloidal stability of the system. In addition, the very small particle size, comparable to the Debye length and the hydrated cations diameters, further contests the applicability of the theory. 

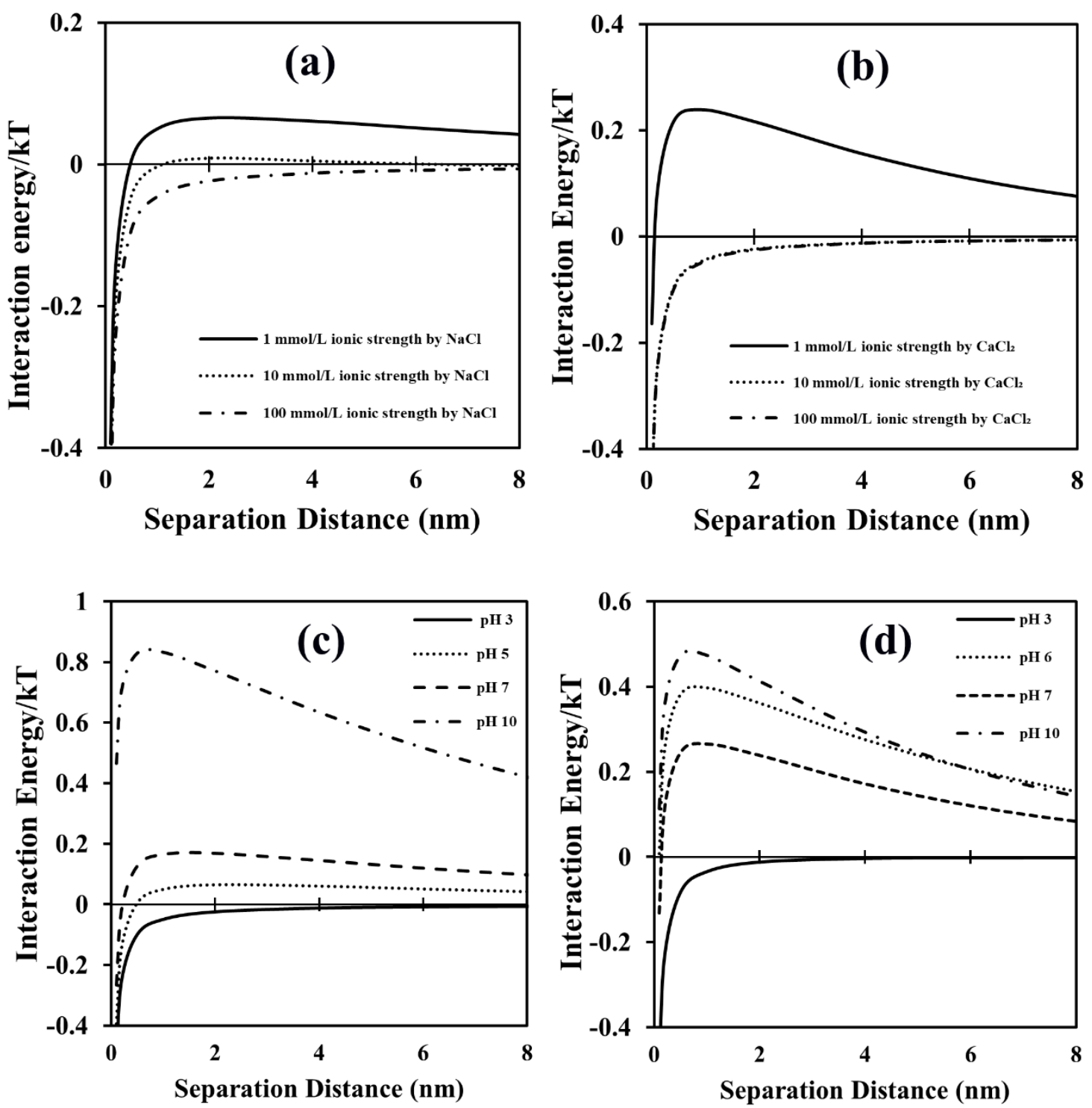

Figure 3.9 Calculated DLVO interaction energy plotted as a function of separation distance between CDs particles; effect of ionic strength and $\mathrm{pH}$ variations. (a) $\mathrm{CDs}$ with different $\mathrm{NaCl}$ concentrations. (b) $\mathrm{CDs}$ with different $\mathrm{CaCl}_{2}$ concentrations. (c) $\mathrm{CDs}$ in $1 \mathrm{mmol} / \mathrm{L} \mathrm{NaCl}$ with different $\mathrm{pH}$. (d) $\mathrm{CDs}$ in $1 \mathrm{mmol} / \mathrm{L} \mathrm{CaCl}_{2}$ with different $\mathrm{pH}$. 

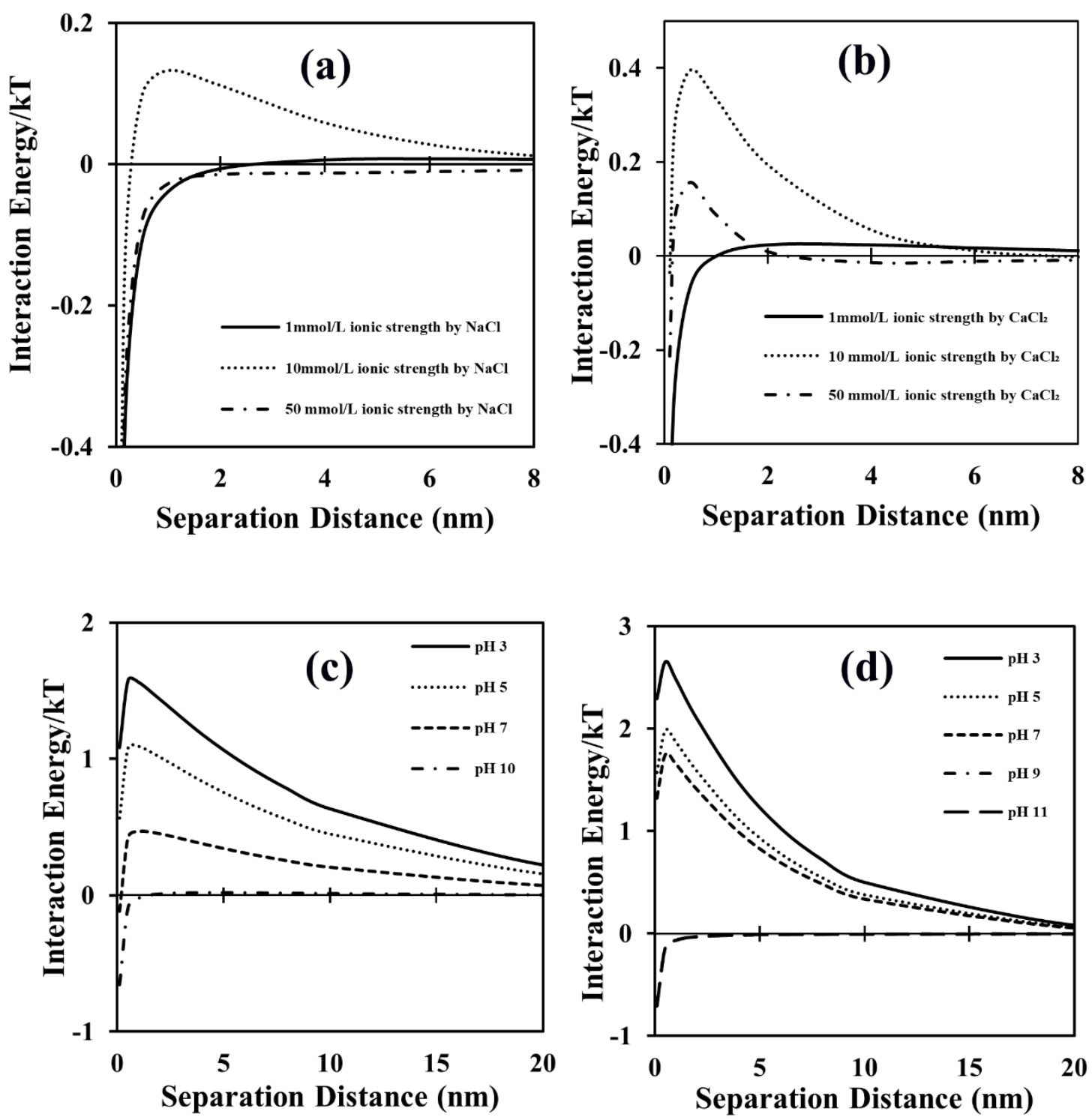

Figure 3.10 Calculated DLVO interaction energy plotted as a function of separation distance between a-CDs particles; effect of ionic strength and $\mathrm{pH}$ variations. (a) a-CDs with different $\mathrm{NaCl}$ concentrations. (b) a-CDs with different $\mathrm{CaCl}_{2}$ concentrations. (c) a-CDs in $1 \mathrm{mmol} / \mathrm{L} \mathrm{NaCl}$ with different $\mathrm{pH}$. (d) a-CDs in $1 \mathrm{mmol} / \mathrm{L} \mathrm{CaCl}_{2}$ with different $\mathrm{pH}$ 


\subsubsection{Fluorescence}

We investigated the fluorescence emission intensity of both nanomaterials at three levels of ionic strength, given by two electrolytes: $\mathrm{NaCl}$ and $\mathrm{CaCl}_{2}$. The experiments were conducted at a constant particle concentration for each type of CD: $200 \mathrm{mg} / \mathrm{L}$ for a-CDs and 52,000 $\mathrm{mg} / \mathrm{L}$ for CDs. Those concentrations were chosen in order to achieve a high initial fluorescence emission intensity. Analysis of emission intensity changes with particle concentration showed that the height of the emission peak increased linearly with concentration up to the chosen particle concentrations, ruling out the potential interference of interparticle interactions, such as aggregation, in the fluorescence determinations (Figure 3.11). The ionic strength results are presented in Figure 3.12. Since aggregations negatively affects the emission, we focused our work on those ionic strength conditions in which we did not observed significant particle size growth in our DLS determinations.
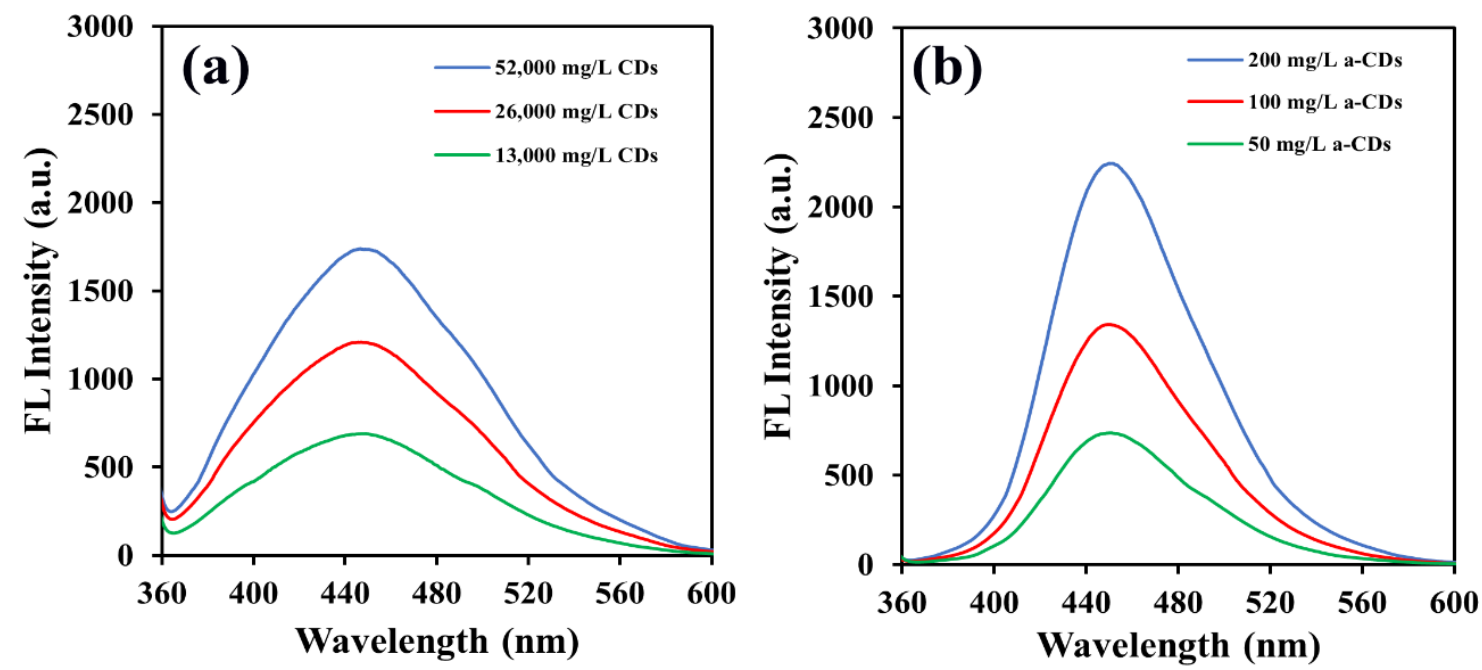

Figure 3.11 Fluorescence spectra for (a) CDs, and (b) a-CDs, at different concentrations. 

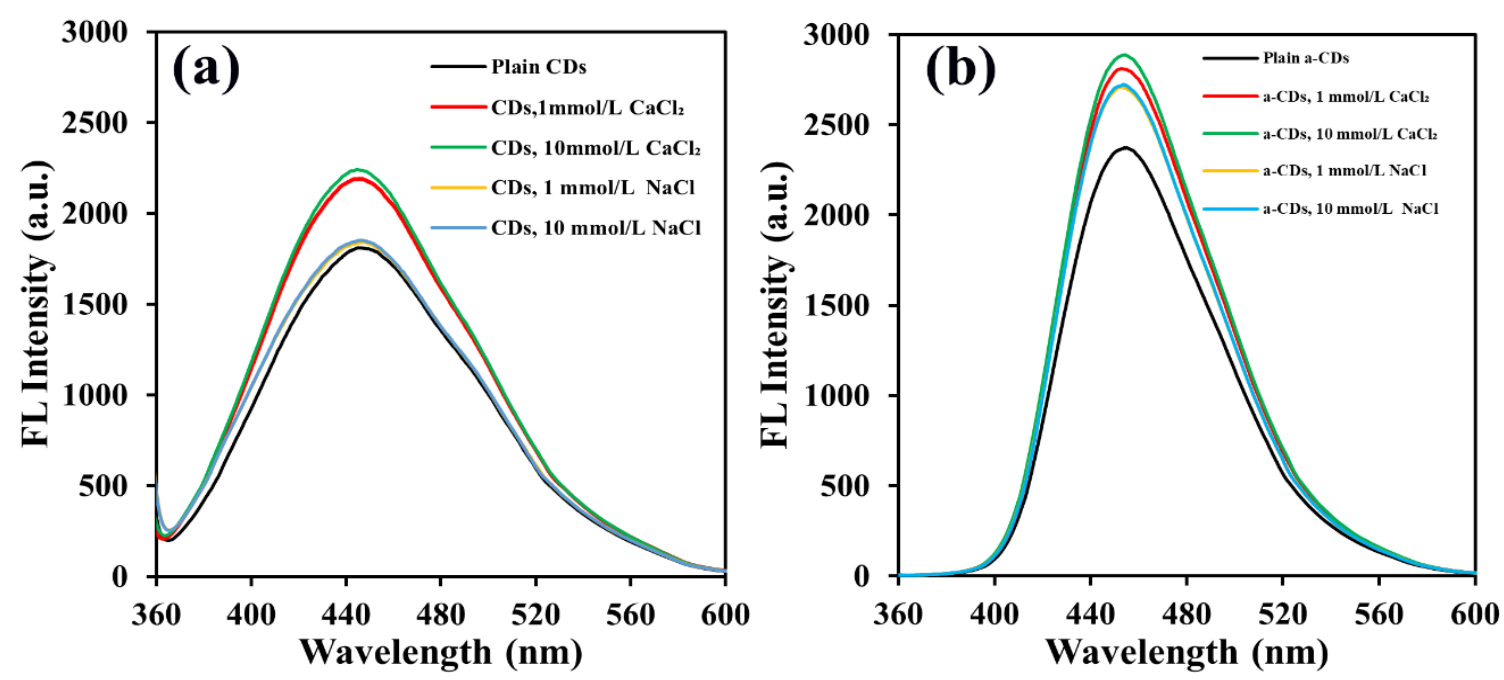

Figure 3.12 Fluorescence spectra for (a) CDs, and (b) a-CDs, at various levels of ionic strength and electrolytes.

Fluorescence emission intensity increased with ionic strength for both CDs and a-CDs in the presence of an indifferent electrolyte. The ionic strength PL behavior implies that the surface defects may serve as capturing centers for carriers, thereby resulting in correlated luminescence [139]. However, different effects were observed for the suspensions prepared in $\mathrm{CaCl}_{2}$. Lower emission intensities were observed for $\mathrm{CDs}$ with increasing concentration of $\mathrm{Ca}^{2+}$ in comparison with the same ionic strength level given by the monovalent ion. However, the presence of calcium resulted in a negligible effect on the a-CDs. The specific adsorption of $\mathrm{Ca}^{2+}$ was much more noticeable on the negatively charged CDs than on the a-CDs; there was, nevertheless some adsorption as indicated by the differences in zeta potential detected for the latter, but it was not strong enough to have an influence on the fluorescence properties of the carbon dots. 
We studied the influence of natural organic matter on fluorescence emission intensity of CDs and a-CDs, using HA as a model compound in concentrations from 1 to $100 \mathrm{mg} / \mathrm{L}$. The PL intensity of pure HA at the concentrations used in the experiment, shown in Figure 3.13, was substantially lower than that of the carbon dots. In the presence of HA, the emission intensity of CDs and a-CDs decreased with increasing concentration of the model compound. Compared to CDs, a-CDs were affected more noticeably. As discussed, HA can be adsorbed on both nanomaterials and the degree of adsorption is dependent on their surface characteristics. Therefore, functionalized a-CDs had higher affinity for adsorption of HA because of the positively charged amino groups on the surface. The better adsorption due to the formation of surface complexes between protonated amino groups and HA resulted in a larger change in emission intensity [138]. Our results suggested that PL from CDs is related to the surface states of the nanoparticles, since adsorption played a major role in controlling emission intensity. However, HA adsorption at the wavelength of emission peak for both carbon dots became significant at high HA concentration (50 and $100 \mathrm{mg} / \mathrm{L}$ ), which would further contribute to the observed quenching. The negative impact by dissolved NOM on CDs and a-CDs has implications for sensing, potentially limiting the applications of CDs in high BOD streams or wastewaters [145]. 

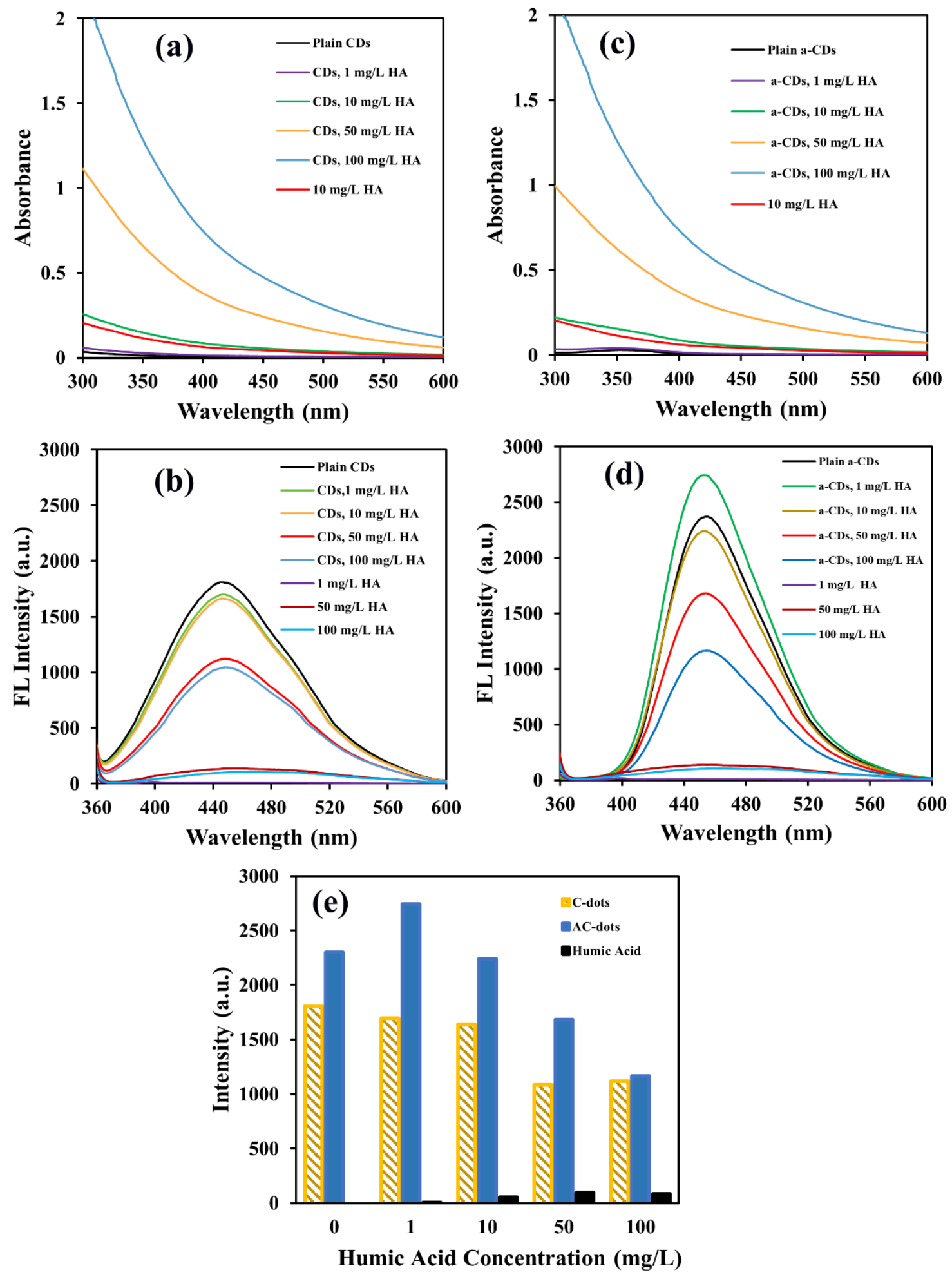

Figure 3.13 Absorption and fluorescence spectra: CDs (a) and (b); a-CDs(c) and (d), respectively, in the presence of HA; comparison of fluorescence intensity peak for CDs, a-CDs suspended in HA solutions (1mg/L-100mg/L) and pure HA (e). 


\subsection{Conclusions}

As prepared, $\mathrm{CDs}$ and a-CDs formed highly stable water suspensions and yielded a circumneutral $\mathrm{pH}$ for $\mathrm{CDs}$ and basic conditions for a-CDs. Negatively charged CDs suspended in $\mathrm{NaCl}$ solutions exhibited behavior compatible with classical colloidal stability principles, but aggregation was inhibited in the presence of $\mathrm{CaCl}_{2}$, even for very low surface charge conditions. a-CDs remained positively charged for all conditions investigated and showed higher zeta potentials at intermediate ionic strength levels followed by a moderate charge decrease but remained stable in suspension. This is in contrast to the general behavior observed for nanoparticles (lower surface charge and aggregation with increasing ionic strength), and hints to the importance of specific adsorption of divalent cations on carbon dots and even monovalent cations for the aminocoated particles. The surface charge of both carbon dots varies with $\mathrm{pH}$ as expected from the acidity constants of their surface functional groups. However, particle size remains fairly constant, always below $50 \mathrm{~nm}$. Although nanoparticles tend to aggregate because of their high surface energies, more strongly so for smaller particles as in the case of carbon dots, coatings (either engineered or adsorbed from the suspension media, e.g. NOM) may hinder aggregation. Aggregation is expected as a result of most typical concentrations of $\mathrm{Ca}^{+2}$ in surface waters regardless of the presence of NOM and under groundwater conditions, $\mathrm{Ca}^{+2}$ and NOM being key factors in nanoparticle stability, in contrast with our findings. If nanomaterials aggregate fast under natural water conditions and settle, they will impact mostly sediments and benthic organisms. We have shown in this work that carbon dots exhibit an opposite behavior, as they will most likely remain suspended in natural waters and will impact animals that inhabit the water column such as planktonic 
species, fish, and marine mammals. Moreover, high stability translates into excellent transport properties, which may lead to impacted ecosystems far from source of contamination. Presence of HA also led to a unique response, inducing aggregation due to bridging for low to moderate concentrations and followed by stabilization at higher levels, independently of surface charge modifications. Interestingly, both positively and negatively charged particles presented an increase in the magnitude of zeta potentials with HA concentration.

Fluorescence emission intensity declines were observed for water chemistry conditions at which specific adsorption of ions or HA was suspected, suggesting surface states as the origin of the PL. However, the adsorption of HA at the peak emission wavelength for the carbon dots may have also contributed to this observation. This phenomenon may limit the application of carbon dots in sensing of natural waters. 


\title{
CHAPTER FOUR
}

\section{Aggregation of Graphene Oxide in Natural Waters: The Role of Solution Chemistry and Specific Interactions}

\begin{abstract}
In this paper, the impact of $\mathrm{pH}$, ionic strength, and dissolved organic matter [humic acid (HA) and tannic acid (TA)] on graphene oxide (GO) stability was investigated. The results showed that the $\mathrm{GO}$ is negatively charged over a $\mathrm{pH}$ range from 2 to $11 . \mathrm{pH}$ did significantly affect GO stability at a level of 4 or higher, but the particles became unstable below $\mathrm{pH} 3$ due to protonation of $-\mathrm{COOH}$ at the edge. Ionic strength (IS) and salt type had observable effects on stability as a result of electrical double layer compression and specific interactions. $\mathrm{CaCl}_{2}$ affects $\mathrm{GO}$ more noticeably than $\mathrm{NaCl}$ because of the binding ability of $\mathrm{Ca}^{2+}$ ions with carboxyl and hydroxyl functional groups. $\mathrm{pH}$ had negligible effects in the presence of $10 \mathrm{mM} \mathrm{NaCl}$, but $1 \mathrm{mM} \mathrm{CaCl} 2$ decreased $\mathrm{GO}$ stability as $\mathrm{pH}$ increased owing to the adsorption of $\mathrm{Ca}^{2+}$ ions on the surface functional groups of GO and the consequent decrease of surface charge. The applicability of DLVO theory as a predictive tool was investigated by modeling GO sheets in two different geometries; three-dimensional sphere like particles and two-dimensional particles at different values of $\mathrm{pH}$ and IS. Overall, the specific interactions and chemical structure of adsorbed organics had a dominant role in GO stability.
\end{abstract}

Keywords: Aggregation; Graphene oxide; Surface chemistry; DLVO theory 


\subsection{Introduction}

Graphene is a two-dimensional carbon-based nanomaterial formed by hexagonal rings of $\mathrm{sp}^{2}$-hybridised carbon atoms $[32,47]$. Studies demonstrated that graphene can be produced using low-cost carbon sources; for instance, insects, waste materials, and food, underscoring the sustainability aspects of the material [146]. The most common forms of graphene are graphene oxide (GO), pristine graphene, and reduced graphene oxide [147]. Graphene has found applications in data storage devices, high-speed electronics, flexible touch screens, electrochemical sensors, solar cells and supercapacitors [148,149]. Graphene oxide (GO) is used in environmental processes, including adsorption, where GOcoated with sand showed higher removal efficiency of heavy metals from water than pure sand [150]. Studies showed that addition of GO may result in membrane water permeability significantly higher than conventional reverse osmosis values [151]. The increasing rate of production of graphene nanomaterials raised concerns about their ecological risks and potential health impact in the early 2010s $[152,153]$. Recent studies showed that graphene nanoparticles can be toxic to humans and bacteria. Liu et al.[57] found that graphene is cytotoxic to bacteria over both oxidative stress and membrane and that GO has the highest antibacterial capacity, pursued by reduced graphene oxide and graphene. A different study [154] reported modest toxicity of GO against zebrafish and human cell lines, while multiwalled carbon nanotubes exhibited intense toxicity toward organisms. Recently, researchers showed that bacteria can be wrapped by graphene sheets and isolated biologically from the environment [155]. As the graphene family of nanomaterials finds more applications in different technological fields, the scenario in which a portion of the aqueous streams containing these nanomaterials end in wastewater treatment plants 
becomes a possibility with the consequential occurrence of interactions between other pollutants in the sewage, nanomaterials, and the microorganisms of the secondary treatment. This could affect the performance of the unit process and have a negative effect in the natural environment if released with the treated water [156]. It is therefore essential to study the behavior of GO in water, in order to assess the risks involved. Pristine graphene (or single layer graphene) is highly hydrophobic and therefore cannot be dispersed in water without the aid of a surfactant $[11,12]$. However, many surface-active compounds can be found in domestic and industrial wastewaters, and therefore graphene dispersion in natural water cannot be completely discarded. Furthermore, natural organic matter (e.g. humic acid) and exopolymeric substances (e.g. proteins and polysaccharides) have been shown to adsorb onto carbon nanotubes and act as stabilization agent [13]. GO, on the other hand, has numerous surface groups such as hydroxyl, carboxyl, and epoxy groups that render it mostly hydrophilic and negatively charged over all environmentally relevant $\mathrm{pHs}[9,14]$, and thus electrostatic interactions prevent individual sheets form aggregating. Increasing ionic strength is expected to induce aggregation through compression of the electrical double layer [15], but this effect is dependent on size and $\mathrm{C} / \mathrm{O}$ ratios, which in turn determine the relative importance of van der Waals attraction to electrostatic repulsion forces [16].

Due to their large surface area and activity, adsorption is expected to play an important role in the fate and transport of carbon-based nanomaterials [9]. In the particular case of graphene, adsorption may alter the surface characteristics of the nanoparticles, making them stable and thus mobile in aqueous media [14]. Organic macromolecules, such as natural organic matter, polysaccharides and proteins, are ubiquitous in natural waters and 
therefore readily available to interact with graphene [13]. Possible inter molecular interactions include $\pi-\pi$ bonding and hydrophobic effects, since the mentioned macromolecules present $\mathrm{C}=\mathrm{C}$ double bonds as well as aromatic rings [17].

A few studies on colloidal stability of GO particles investigated the effect of $\mathrm{pH}$, cations, and natural organic matter (NOM); while most recognized the complex interplay of those solvent water chemistry, they failed to provide a comprehensive assessment of their effects $[74,81,157,158]$. Aggregate shape, and chemical properties (amount of oxygen containing groups, reducing agent used in fabrication) of the GO as well as of the NOM surrogate selected (humic acid, Suwanee River HA, polyacrylic acid, natural water samples) seemed to influence aggregation behavior, precluding comparison of data between studies and leading in some cases to conflicting conclusions.

The objective of this work is to investigate the effects of the GO surface physical and chemical transformations induced by the water chemistry on the aggregation behavior of the GO sheets. GO suspensions in the presence of monovalent and divalent cations at variable concentrations were studied for the full range of environmentally relevant pHs. The role of NOM chemical composition was evaluated, selecting humic acid and tannic acid as model compounds. Experimental results were compared to predictions by classic DLVO model of colloidal interactions. GO sheets have been largely modeled as spherical particles, regardless of their recognized 2D geometry. Gudarzi [159] proposed a set of equations for the calculation of interacting energies of thin layers. However, GO is not a rigid particle and it is expected to bend and crumple when subjected to the attractive forces during aggregation, leading to an intermediate and variable dimensionality as the 
aggregation process progresses. The applicability of the DLVO model as a predictive tool and the geometry of the modeled system is discussed.

\subsection{Materials and methods}

\subsubsection{Synthesis}

GO was prepared from graphite nanoplatelets (5um, $\mathrm{xGnP}$ graphite nanoplatelet, $\mathrm{XG}$ Sciences) following a modified Hummer's method [18]. In brief, $1 \mathrm{~g}$ of graphite and $1 \mathrm{~g}$ of $\mathrm{NaNO}_{3}$ (Sigma-Aldrich) were mixed together in an ice-bath followed by adding $46 \mathrm{ml}$ of 98\% (w/w) $\mathrm{H}_{2} \mathrm{SO}_{4}$ (Fisher Scientific). Then, $6 \mathrm{~g}$ of $\mathrm{KMnO}_{4}$ (Flinn Scientific) were carefully added into the mixture. After stirring for $1 \mathrm{~h}$ at $35^{\circ} \mathrm{C}, 80 \mathrm{~mL}$ of ultrapure water $(18 \mathrm{M} \Omega . \mathrm{cm})$ were added carefully to the mixture and the temperature was increased to $90{ }^{\circ} \mathrm{C}$ for $30 \mathrm{~min}$, followed by the addition of $200 \mathrm{ml}$ of ultrapure water and $6 \mathrm{ml}$ of $30 \% \mathrm{H}_{2} \mathrm{O}_{2}$ (Fisher Scientific). The resulting mixture was washed with DI water for at least 20 times to obtain $\mathrm{pH}$ (4-5). Finally, the GO was obtained by exfoliation under ultrasonication for $45 \mathrm{~min}$ (42 $\mathrm{kHz}, 2510$, Branson).

\subsubsection{Characterization}

Morphology was observed by Transmission Electron Microscopy (TEM, JEOL 1400) with an accelerating voltage of $120 \mathrm{kV}$. The thickness of GO sheets was measured using Atomic Force Microscopy (AFM) (Nanoscope IIIa). A detailed description of the sample preparation methods is presented in the Supporting Information.

The surface chemistry of graphene and GO was analyzed by Fourier-Transform 
Infrared Spectroscopy (FTIR, Nicolet 4700) with $\mathrm{KBr}$ as a background. The BrunauerEmmett-Teller (BET) surface area for the GO was measured by (SA 3100, Beckman Coulter, USA). The crystalline structure and the interlayer spacing (d-spacing) of GO were studied by X-Ray Diffraction (XRD) (PANanlytical X'Pert Pro, Netherlands). The samples were scanned from $5^{\circ}$ to $60^{\circ}(2 \theta)$ with a step size of 0.025 and account time of $1 \mathrm{~s}$ at each point. Finally, X-ray photoelectron spectroscopy (XPS) was performed in an ultrahigh vacuum (Kratos Axis 165) using a monochromatic Al $\mathrm{Ka}$ source (hv $=1486.6 \mathrm{eV}$, resolution $=0.5 \mathrm{eV}$ ). The hydrodynamic properties of $\mathrm{GO}$ were measured under different salt types $\left(\mathrm{NaCl}, \mathrm{CaCl}_{2}\right)$ and wide range of ionic strength (IS), $\mathrm{pH}$, and natural organic matter (NOM). Two compounds were used as models of dissolved organic material in natural water: humic acid (HA) (Technical Grade; Acros, Geel, Belgium) and tannic acid (TA) (Sigma-Aldrich).

The influence of $\mathrm{pH}$ on stability of GO was investigated over a $\mathrm{pH}$ range from 2 to 11 in the presence of $\mathrm{NaCl}$ and $\mathrm{CaCl}_{2} . \mathrm{HCl}$ (Fisher Scientific) and $\mathrm{NaOH}$ (Fisher Scientific) were used for $\mathrm{pH}$ adjustments. A ZetaSizer Nano ZS (Malvern Instruments, Worcestershire, U.K.) was used to measure hydrodynamic diameter $\left(D_{h}\right)$, and zeta potential

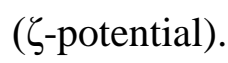

In order to characterize the electrophoretic mobility (EPM) of the nanomaterials in the various electrolyte solutions using a Malvern Zetasizer Nano ZS, laser Doppler velocimetry was applied. Measured EPMs were converted to zeta-potential using the Smoluchowski equation (Equation 4.1) [135]:

$$
U=\frac{\varepsilon \zeta}{\mu}
$$


where $U$ is the electrophoretic mobility, $\varepsilon$ is the dielectric constant of the solution, $\mu$ is its viscosity, and $\zeta$ is the zeta potential. Disposable folded capillary cells were employed.

\subsubsection{Theory}

The total interaction energy between colloidal particles ( $\left.V_{\text {тотАL }}\right)$ was calculated to predict the aggregation state, as described by traditional DLVO theory. According to this theory, the stability of colloidal particles in aqueous environments can be determined by the sum of van der Waals interactions $\left(V_{V d W}\right)$ and electrical double layer interactions $\left(V_{E D L}\right)$ [134].

$$
V_{\text {Total }}=V_{E D L}+V_{v d w}
$$

\subsubsection{Sphere-Sphere DLVO interaction}

In a first approach, GO particles were modeled as spherical in shape with size equal to their hydrodynamic diameter. Since dispersion forces are electromagnetic in character, they are subject to a retardation effect [135]. Thus, van der Waals interactions were calculated with retardation (Equation 4.3):

$$
V_{v d w}=-\frac{A_{i w i} a_{P}}{12 h}\left[\left(1+\frac{14 h}{\lambda}\right)\right]^{-1}
$$

Where $a_{P}$ is the radius (m); $\mathrm{h}$ is the surface-to-surface distance between two particles (m); $\mathrm{A}_{\text {iwi }}$ is the Hamaker constant, $49 \times 10^{-21} \mathrm{~J}$ was employed in this study [159]; $\lambda$ is the characteristic wavelength of the dielectric and usually assumed $100 \mathrm{~nm}$. 
The electrostatic double layer interactions were calculated using the following expressions (Equation 4.4) [135]:

$$
V_{E D L}=\frac{64 \pi a_{P} n_{\infty}}{K^{2}} k_{B} T \gamma^{2}[\exp (-K h)]
$$

$n_{\infty}$ is bulk number density of ions which is calculated according to Equation (4.5)

$$
n_{\infty}=1000 \times N_{A} \times C_{s}
$$

where $\mathrm{C}_{\mathrm{S}}$ is the electrolyte molar concentration and $N_{A}=6.02 \times 10^{23}(1 / \mathrm{mol})$ is Avogadro's number, $k_{B}=1.38 \times 10^{-23}(\mathrm{~J} / \mathrm{K})$ is Boltzmann constant and $T=298(\mathrm{~K})$ is absolute temperature. The parameter $\gamma$ is calculated according to Equation (4.6),

$$
\gamma=\operatorname{Tanh}\left(\frac{z e \zeta}{4 k_{B} T}\right)
$$

where $z$ is the charge of the ions, $\zeta$ is the experimental zeta potential $(\mathrm{V}), e=1.602 \times 10^{-19}$ Coulombs is the elementary charge [3], and $K$ is reciprocal of the Debye screening length $\left(\mathrm{m}^{-1}\right)$ as calculated in Equation (4.7).

$$
K=\sqrt{\frac{2 N_{A} e^{2} 1000 I}{\varepsilon \varepsilon_{0} k_{B} T}}
$$

where $\mathrm{I}(\mathrm{mol} / \mathrm{L})$ is the ionic strength defined as $(1 / 2) \Sigma \mathrm{z}_{\mathrm{i}}^{2}\left[\mathrm{x}_{\mathrm{i}}\right] ; \mathrm{x}_{\mathrm{i}}$ is the molar concentration of the ith species, and $z_{i}$ its valency, $\varepsilon$ is the dielectric constant of solvent, $\varepsilon_{0}$ is the permittivity of vacuum. These expressions are valid for $\mathrm{h}<<$ ap and Kap $>5$. 


\subsubsection{Plate-Plate DLVO interaction}

In a second approach, we modeled van der Waals interactions $\left(V_{V d W}\right)$ between GO sheets as thin plates using the following Equation (4.8) [135,159]:

$$
V_{v d w}=-\frac{A_{i w i}}{12 \pi}\left[\frac{1}{h^{2}}+\frac{1}{\left(h+2 \delta_{p}\right)^{2}}-\frac{2}{\left(h+\delta_{p}\right)^{2}}\right]
$$

where $\delta_{p}$ is the thickness of the plate $(\mathrm{m})$.

The electric double layer (EDL) interactions between two GO sheets was modeled using linearized Poisson-Boltzmann approximation for two planar surfaces as follows:

$$
V_{E D L}=\frac{2 \sigma^{2}}{\varepsilon \varepsilon_{0} K} \exp (-K h)
$$

where $\sigma$ is the surface charge density and can be calculated using Equation (4.10) [159]:

$$
\sigma=\sqrt{8 \varepsilon \varepsilon_{0} k_{B} T} \sinh \left(e \xi / 2 k_{B} T\right)\left\{[\mathrm{NaCl}]+\left[\mathrm{CaCl}_{2}\right]\left(2+\exp \left(-e \xi / k_{B} T\right)\right\}^{1 / 2}\right.
$$

where the concentration of $\mathrm{NaCl}$ and $\mathrm{CaCl}_{2}$ is expressed in moles per liter.

\subsection{Results and discussion}

\subsubsection{GO Characterization}

Figure 4.1 (a) in supporting information shows a representative TEM image of a GO particle, evidencing its two-dimensional morphology under conditions of low $\mathrm{pH}$ and 
absence of added electrolytes, but folded aggregated 3-D structures were observed for particles suspended in $0.5 \mathrm{mM} \mathrm{Ca}^{2+}$ solutions (Figure $4.1(\mathrm{~b}, \mathrm{c})$ ). The thickness of individual GO particles was estimated from AFM scans (Figure 4.1 (d)), where more than 20 individual sheets were measured. The results showed an average thickness of $1.2 \pm 0.2$ nm.

The FTIR spectra of graphite and GO is presented in Figure 4.2 (a). As expected, graphite did not show any significant peaks, but different types of oxygen functionalities were confirmed in the GO spectrum: at $3300 \mathrm{~cm}^{-1}\left(\mathrm{O}-\mathrm{H}\right.$ stretching vibration), at $1740 \mathrm{~cm}^{-}$ ${ }^{1}$ (stretching vibration from $\mathrm{C}=\mathrm{O}$ ), at $1619 \mathrm{~cm}^{-1}\left(\mathrm{C}=\mathrm{C}\right.$ stretch), at $1360 \mathrm{~cm}^{-1}$ (C-O stretch), at $1201 \mathrm{~cm}^{-1}$ (C-OH stretching vibration), and finally at $1060 \mathrm{~cm}^{-1}$ (C-O stretching vibration). 

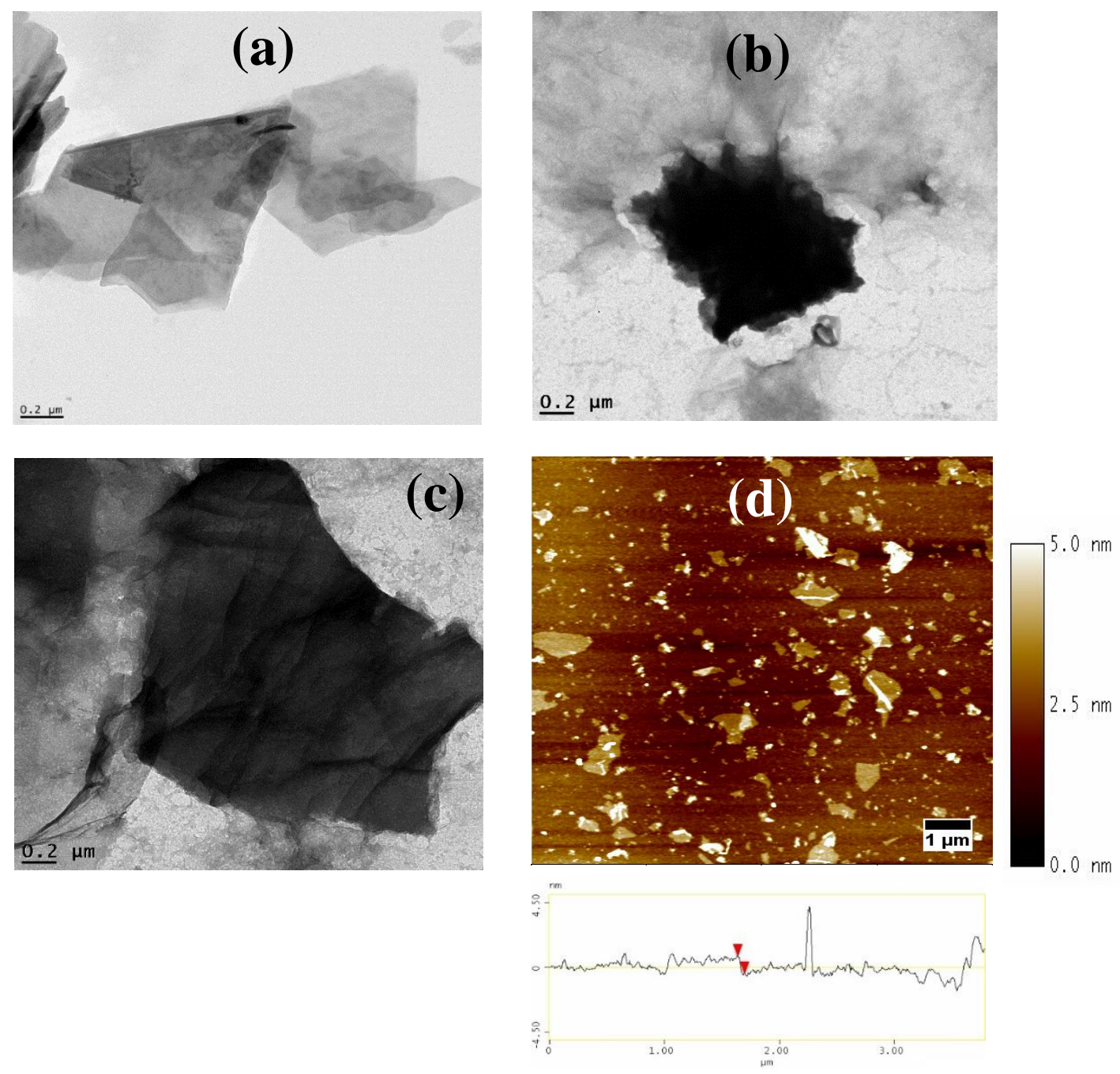

Figure 4.1 Representative transmission electron microscopy (TEM) image of GO (a) 2D structure in absence of ionic strength, (b) 3D aggregated structure, (c) folded, aggregated structure. (d) Atomic Force Microscopy (AFM) of GO.

XRD patterns (Figure 4.2 (b)) showed a strong peak at $2 \theta=10.6^{\circ}$ which indicates the (001) reflection of GO. The interlayer spacing was calculated to be $0.84 \mathrm{~nm}$, demonstrating the hydration and exfoliation of graphite after the introduction of functional groups; the interlayer spacing of graphite oxide is larger than that of graphite due to intercalation 
caused by water molecules [160]. The UV-visible adsorption spectra of the GO sheets showed a characteristic peak at $227 \mathrm{~nm}$ (Figure 4.3) due to the $\pi \rightarrow \pi^{*}$ transition of the $\mathrm{C}=\mathrm{C}$ bonds [161].
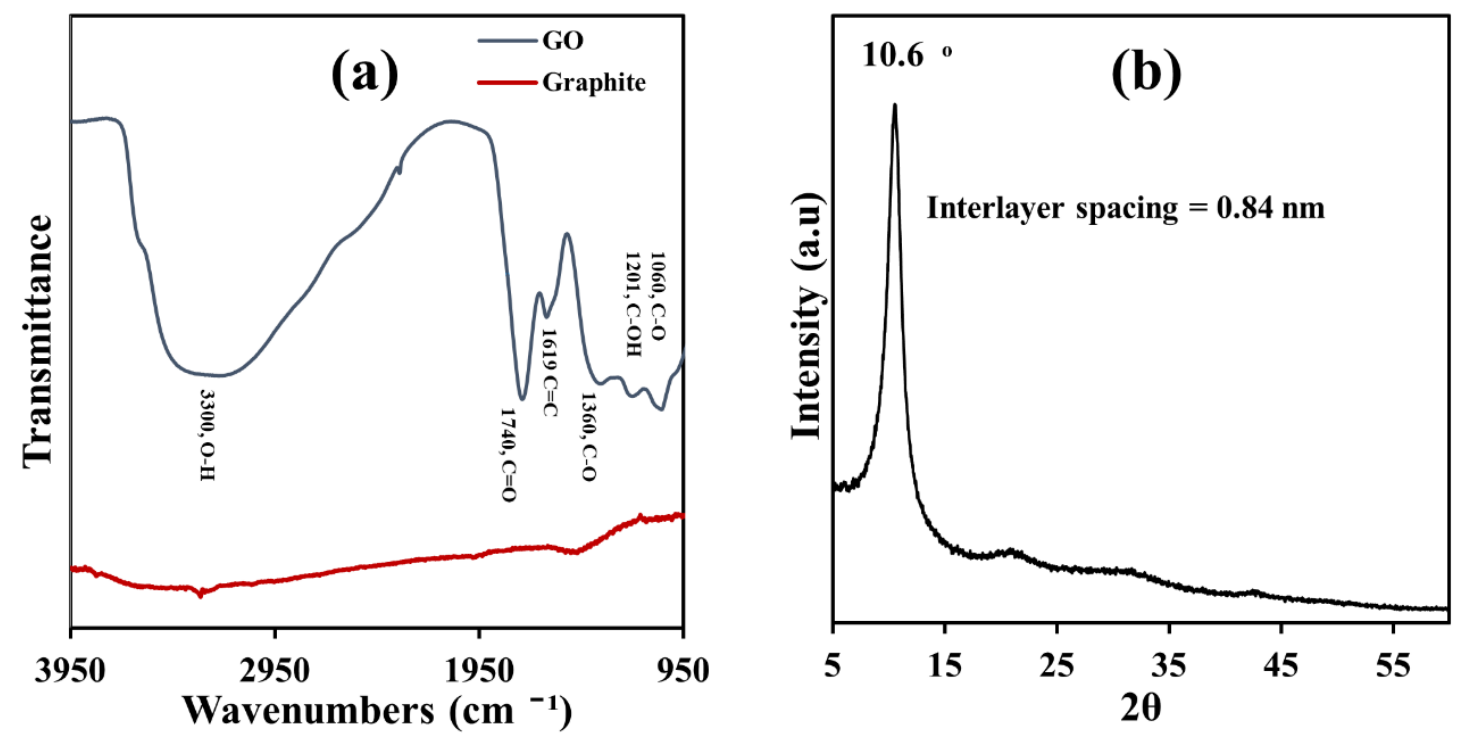

Figure 4.2 (a) Fourier-Transform Infrared Spectroscopy (FTIR) of graphite and GO. (b) X-ray diffraction patterns of GO.

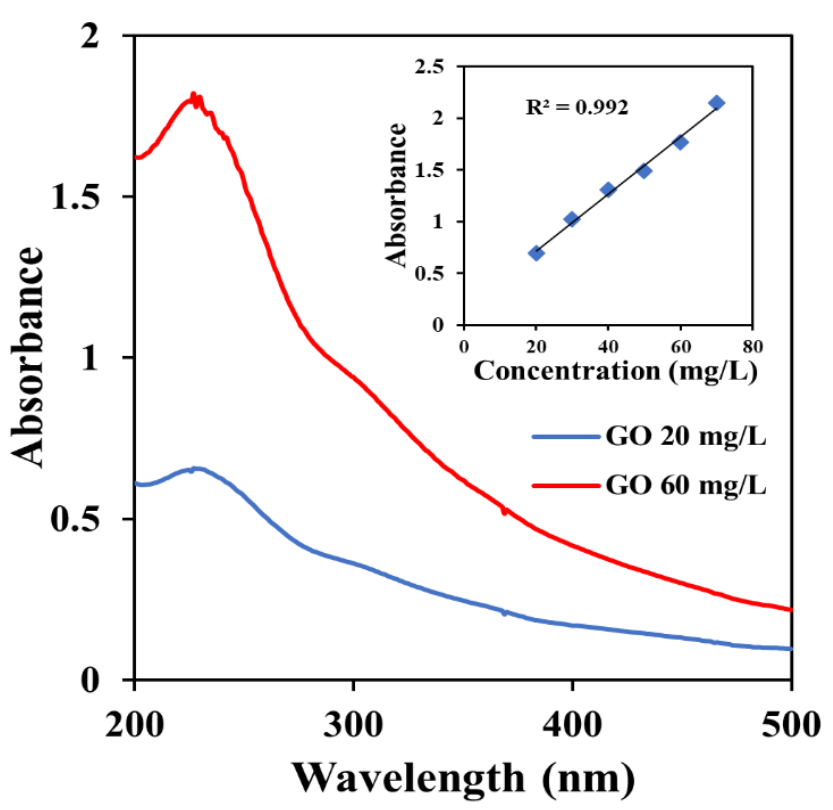

Figure 4.3 UV-Vis absorption spectra and standard curve (insert) of graphene oxide. 
XPS elemental composition analysis showed that the oxygen percentage of GO was $40.3 \pm 0.3 \%$ and carbon percentage $57.5 \pm 0.5 \%$ (Figure 4.4 (a)). A small amount of sulfur $(0.82 \pm 0.02 \%)$ persisted on GO sheets which is probably due to the use of $\mathrm{H}_{2} \mathrm{SO}_{4}$ during GO preparation. XPS results showed that the $\mathrm{C} 1 \mathrm{~s}$ spectrum contains three major peaks after deconvolution, which are assigned as $\mathrm{sp}^{2}$-hybridized carbon at $284.1 \mathrm{eV}$, epoxide (CO-C) and hydroxyl $(\mathrm{C}-\mathrm{OH})$ at $286.1 \mathrm{eV}$, and carboxyl $(\mathrm{C}=\mathrm{O})$ at $287.9 \mathrm{eV}$ (Figure $4.4(\mathrm{~b})$ ). GO obtained in this work had a BET surface area of $158 \mathrm{~m}^{2} / \mathrm{g}$.
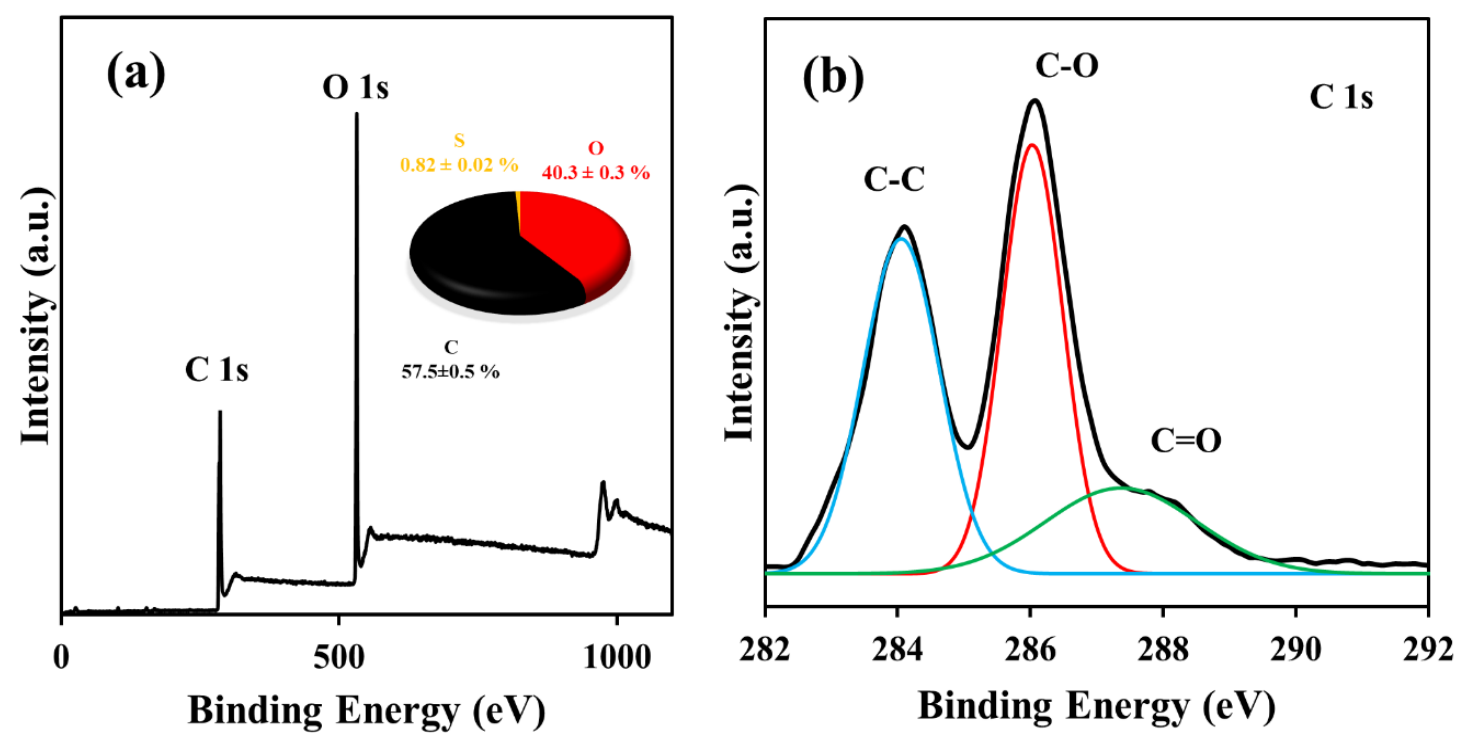

Figure 4.4 XPS spectra of GO. (a) Survey XPS and (b) C 1s XPS spectra

\subsubsection{Aggregation of GO Particles}

The stability of colloids is affected by the solution chemistry. The major driving force preventing aggregating is the electrostatic repulsion [16], as it outweighs attractive forces, mainly van der Waals, in stable suspensions. Negatively charged colloids having zeta potential lower than $-30 \mathrm{mV}$ are generally considered to be electrostatically stable [162]. 
We investigated $D_{h}$ and $\zeta$-potential of GO under a variety of $\mathrm{pH}$, IS, and dissolved organic matter conditions in order to understand their colloidal stability in water suspension. A concentration of $10 \mathrm{mg} / \mathrm{L}$ of $\mathrm{GO}$ was used in all aggregation experiments.

\subsubsection{Impact of Salt Types and Ionic Strength}

The effect of dissolved solids (ionic strength and chemical nature of the electrolytes) was investigated in the presence of $\mathrm{NaCl}$ as model for a monovalent, indifferent electrolyte and $\mathrm{CaCl}_{2}$ as a model for divalent and potentially specific interacting cations, at $\mathrm{pH}$ of 5 . Figure 4.5 illustrates the effect of IS and salt type $\left(\mathrm{NaCl}, \mathrm{CaCl}_{2}\right)$ on $\zeta$-potential of $\mathrm{GO}$. GO remained negatively charged for all $\mathrm{NaCl}$ and $\mathrm{CaCl}_{2}$ concentrations tested. Figure 4.5 (a)) shows that $\zeta$-potential values of GO became less negative, from $-43.23 \pm 1.06$ to $-28.23 \pm$ $1.15 \mathrm{mV}$, as the concentration of $\mathrm{NaCl}$ increased from 1 to $100 \mathrm{mM}$. The increased charges are likely a result of the compression of the electrical double layer, as predicted by the colloidal theory $[9,132,134]$. The diminishing magnitude of the zeta potential is correlated with a significant increase in $D_{h}$ of GO from $435.57 \pm 35.23$ to $2130.4 \pm 120 \mathrm{~nm}$ (Figure 4.6 (a)). In most natural water bodies, including ground and surface water, the concentration of monovalent positive ions $\left(\mathrm{K}^{+}, \mathrm{Na}^{+}\right)$is lower than $10 \mathrm{mM}$ [9], representing that GO could be stable in natural water environments, if dissolved solids are predominantly represented by these cations. Monovalent cations are not expected to develop specific interactions with GO surface functional groups, and the aggregation of GO in existence of $\mathrm{Na}^{+}$can be described by the charge screening theory [135]. Increasing the amount of electrolyte can therefore provide additional protection to the charge surface 
to decrease the electric repulsive forces between colloids and, thus, facilitate their aggregation [70].

The $\zeta$-potential and hydrodynamic diameter of GO particles in the existence of a divalent cation $\left(\mathrm{Ca}^{+2}\right)$ are illustrated in Figure 4.5 (b) and Figure 4.6 (b). The $\mathrm{Ca}^{2+}$ ion had a stronger influence on both $\zeta$-potential and hydrodynamic than the monovalent $\mathrm{Na}^{+}$, when present at equal molar concentrations. Figure 4.1 (b) shows that $\zeta$-potential values of GO are significantly affected by $\mathrm{Ca}^{2+}$, which became less negative from $-21.8 \pm 2.1$ to $-2.73 \pm$ $0.55 \mathrm{mV}$ as $\mathrm{CaCl}_{2}$ concentration increased from 1 to $100 \mathrm{mM}$. This is in part due to the higher charge screening from divalent ions [9][132]. As a consequence, aggregation was observed, and the hydrodynamic diameter $D_{h}$ of GO increased to $1,084.4 \pm 181,1,125.5 \pm$ 161 , and $1,601.3 \pm 169 \mathrm{~nm}$ in presence of $0.1,0.5$, and $1 \mathrm{mM} \mathrm{CaCl}_{2}$ respectively (Figure 4.6 (b)).
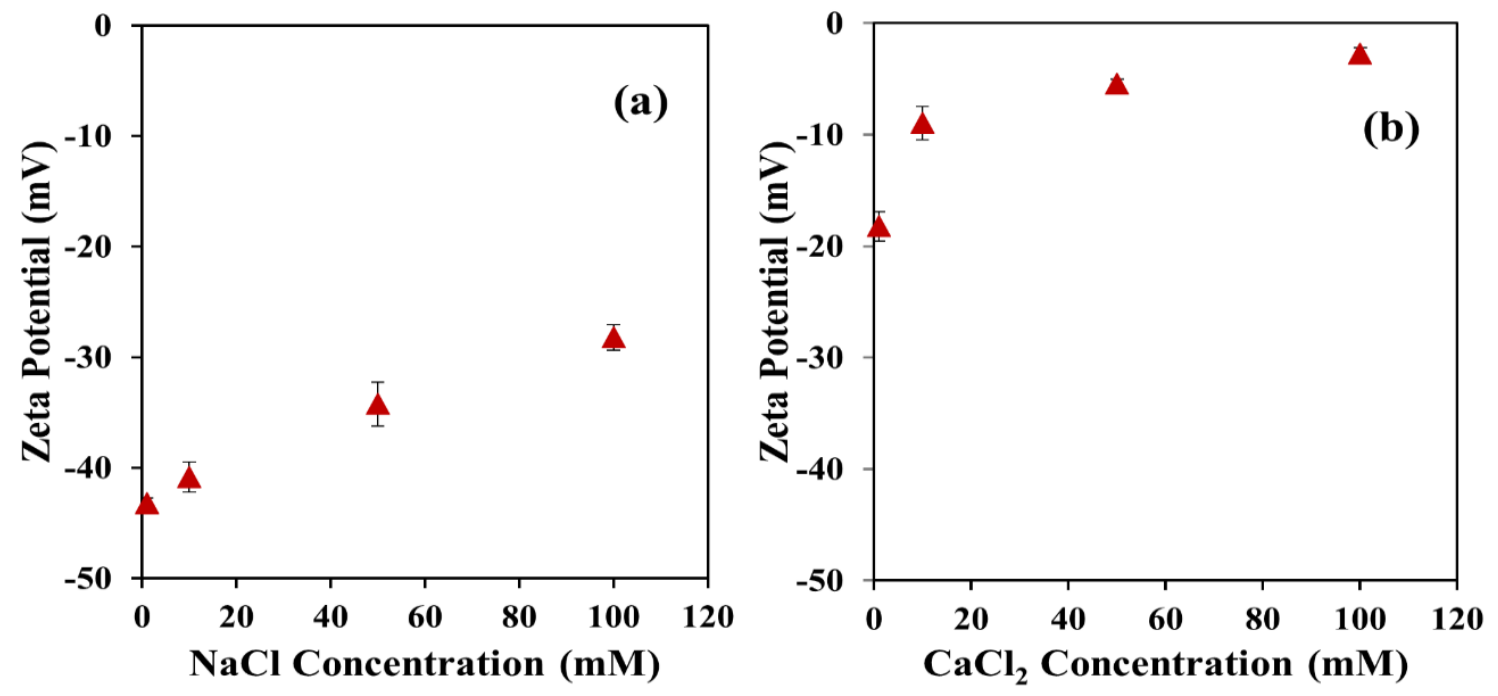

Figure 4.5 Zeta potential of $\mathrm{GO}$ as a function of ionic strength $\left(\mathrm{NaCl}\right.$ and $\left.\mathrm{CaCl}_{2}\right)$ at $\mathrm{pH}$ 5.2. 

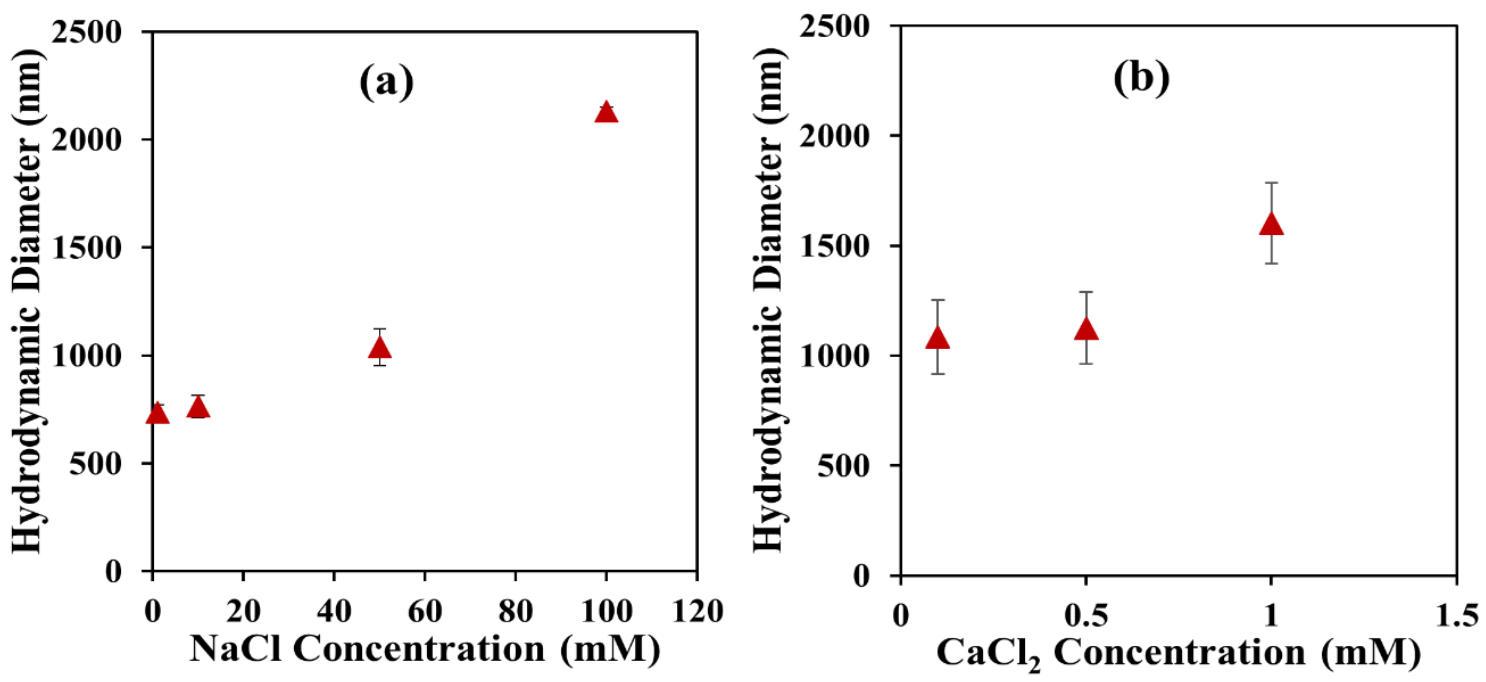

Figure 4.6 Hydrodynamic diameter of $\mathrm{GO}$ as a function of ionic strength $\left(\mathrm{NaCl}\right.$ and $\left.\mathrm{CaCl}_{2}\right)$ at pH 5.2.

These results stress the significant influence of divalent cations in the stability of GO. It is clear that $\mathrm{Ca}^{2+}$ had a strong interaction with $\mathrm{GO}$ functional groups, beyond electrostatic interactions. Furthermore, the FTIR spectra (Figure 4.2 (a)) showed that GO contained carboxyl and hydroxyl functional groups which may be accountable for the change in GO $\zeta$-potential values. Divalent cations may interact with GO functional groups and bridge two individual GO sheets via complexation [163], enhancing aggregation. Studies showed that $\mathrm{Ca}^{2+}$ forms complexes with carboxylic acids [164], and with carboxyl groups on humic and fulvic acid, as well as with GO [165], that were responsible for the increased $\zeta$ - potential and may even cause a charge reversal (Figure 4.5 (b)). Furthermore, Wu et al.[70] proposed three kinds of cross-linking interactions between GO sheets and $\mathrm{Ca}^{+2}$ : (1) cross-linking of the interlamellar water molecules and the H-bonds formed among the oxygen functional groups on GO surfaces, (2) interpolating between the basal planes by either dative bonds 
from hydroxyl and carbonyl groups or weak alkoxide, and (3) bridging the edges of the GO sheets by chelating carboxylate functional groups. All three interactions are feasible mechanisms for $\mathrm{Ca}^{2+}$ induced aggregation in this system. However, $\mathrm{Na}^{+}$ions were only electrostatically adsorbed in the diffuse layer, a much weaker, reversible interaction.

Given the ubiquitous presence of calcium ions in natural waters, the results imply a clear tendency of GO to form aggregates when released to the environment. Furthermore, the aggregates seem to deviate from the 2-D morphology of the as-prepared nanoparticles, as suggested by TEM images.

\subsubsection{Impact of $\mathrm{pH}$}

The stability of GO particles is expected to be altered by the $\mathrm{pH}$ of the solvent, as it regulates the acid-base equilibrium of surface groups. Figure 4.7 shows that the $\zeta$ potential of GO particles is negative over the $\mathrm{pH}$ range from 2 to 11 , both in the presence of $\mathrm{NaCl}$ and $\mathrm{CaCl}_{2}$. For these experiments, a concentration of $0.5 \mathrm{mM} \mathrm{CaCl}_{2}$ was used for size measurement due to fast aggregation at higher $\mathrm{Ca}^{2+}$ concentration and rapid settling of the aggregates formed, making reliable measurements unfeasible. In the presence of 10 $\mathrm{mM} \mathrm{NaCl}, \zeta$-potential values decreased from $-23.17 \pm 0.46$ to $-54.1 \pm 2.45 \mathrm{mV}$ as a $\mathrm{pH}$ increased from 2 to 11 due to the deprotonation of surface functional groups [9]. The $\zeta$ potential vs. pH curve (Figure 4.7 (a)) displayed two inflection points, located at around pH 4 and $\mathrm{pH}$ 9. FTIR spectra (Figure 4.2 (a)) showed that GO has two types of acidic groups: carboxylic (pKa, 4.3) and hydroxylic (pKa, 9.3); increased negative charges on GO sheets resulted from the ionization of these two groups [161]. However, size 
measurements (Figure 4.8 (a)) evidenced a negligible influence of $\mathrm{pH}$ on GO stability from $\mathrm{pH}$ 4-11 in presence of $\mathrm{NaCl}$. In that range, the $D_{h}$ was approximately $520 \mathrm{~nm}$, but increased sharply as $\mathrm{pH}$ further decreased due to the change in $\zeta$-potential and corresponding repulsive forces reduction (Chowdhury et al. 2013; Terracciano et al. 2017).
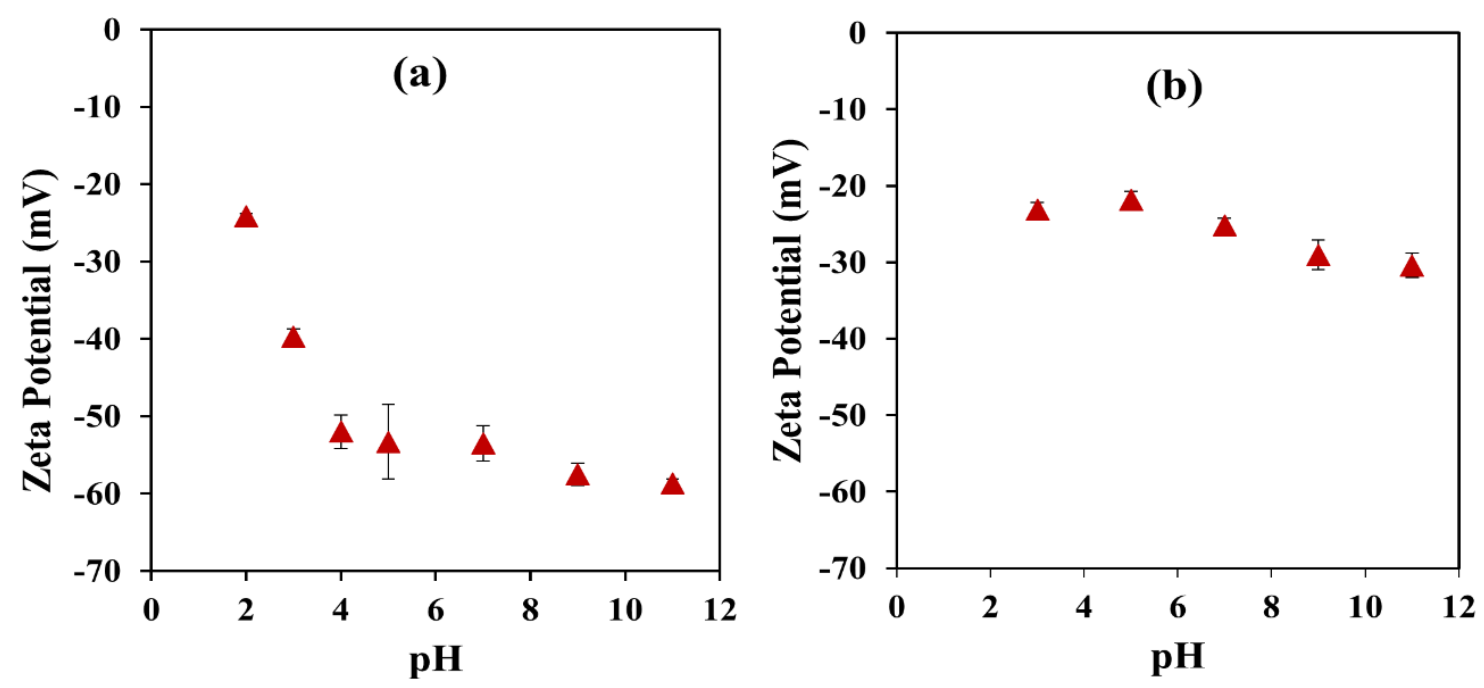

Figure 4.7. Zeta potential of $\mathrm{GO}$ as a function of $\mathrm{pH}$ : (a) $\mathrm{GO}$ in $10 \mathrm{mM} \mathrm{NaCl}$; (b) $\mathrm{GO}$ in $0.5 \mathrm{mM}$ $\mathrm{CaCl}_{2}$.

The normal range of $\mathrm{pH}$ in the natural waters is between 5 and 9 [9][166]; since there were no prominent changes in hydrodynamic properties of $\mathrm{GO}$, it is possible that $\mathrm{pH}$ have only a minor effect on its fate and transport in the presence of indifferent electrolytes. However, when suspended in a $0.5 \mathrm{mM} \mathrm{CaCl}_{2}$, the $\mathrm{GO} \zeta$-potential slightly decreased, from $-23.17 \pm 0.29$ to $-30.40 \pm 0.37 \mathrm{mV}$, as $\mathrm{pH}$ increased from 2 to 11 (Figure 4.7 (b)), while the $D_{h}$ changed from $579.2 \pm 57$ to $2,120.67 \pm 500 \mathrm{~nm}$ (Figure $8(\mathrm{~b})$ ). The adsorption of $\mathrm{Ca}^{2+}$ significantly increased with increasing $\mathrm{pH}$, similarly to the behavior that is typically observed for cations on metal oxide surfaces [167]. Moreover, $\mathrm{Ca}^{2+}$ is known to bridge ionized $\mathrm{OH}$ groups on mineral surfaces (e.g. aluminosilicates, goethite and kaolinite) and 
deprotonated carboxyl groups on organic matter through formation of complexes that involve electrostatic attraction between the cations and negatively charged functional groups [168], inducing aggregation through bridging of groups in two different particles.
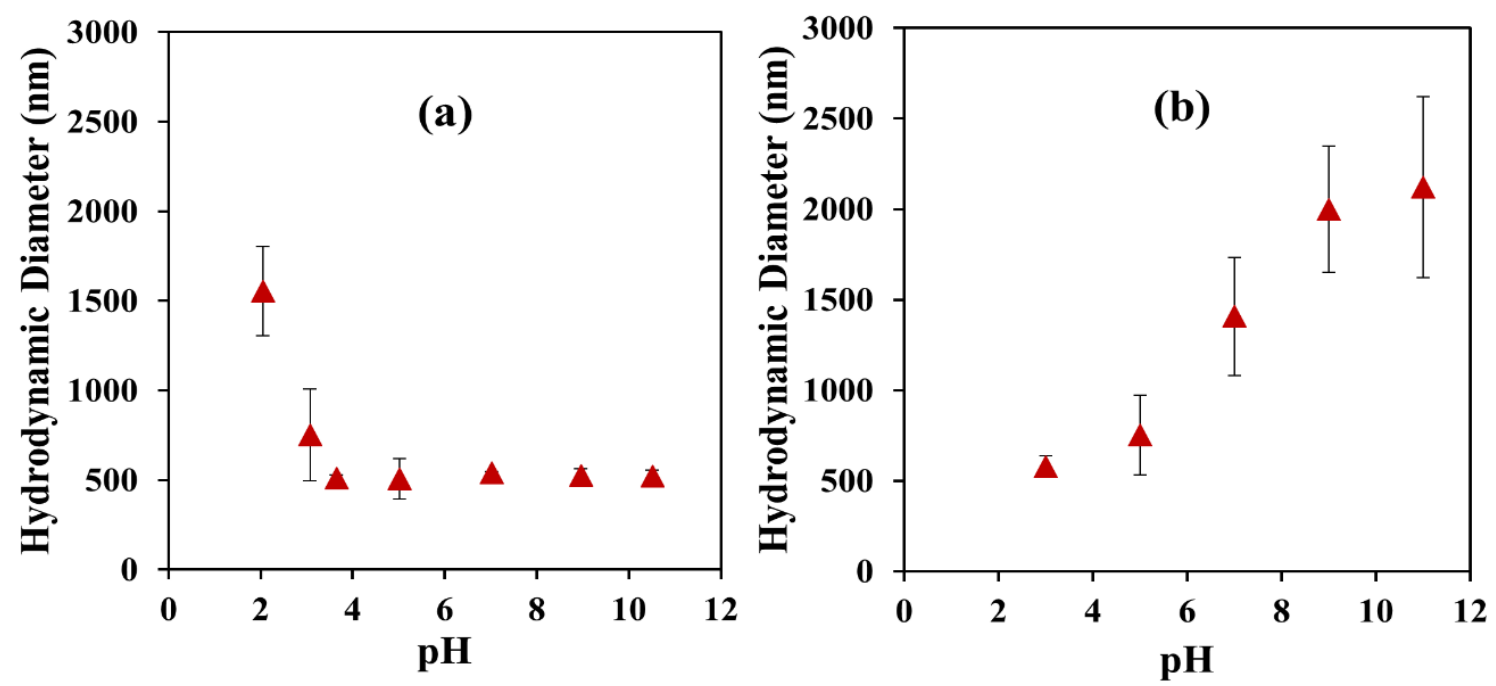

Figure 4.8 Hydrodynamic diameter of GO as a function of pH (a) GO in10 mM NaCl. (b) GO in $0.5 \mathrm{mM} \mathrm{CaCl}_{2}$.

More alkaline $\mathrm{pHs}$ resulted in higher degree of deprotonation of GO functional groups which in turn led to greater binding of $\mathrm{Ca}^{2+}$ and thus, increased the surface charge [65]. The hydroxyl- $\mathrm{Ca}^{2+}$ complex on the basal plane is unstable; a single $\mathrm{Ca}^{2+}$ ion can remove two $-\mathrm{OH}$ groups from the surface and forming $\mathrm{Ca}(\mathrm{OH})_{2}[169]$. As $\mathrm{Ca}^{2+}$ can bind to $-\mathrm{OH}$ in the basal plane, removing it from the graphene oxide plane, the cation can act as a partial healer of the oxidized surface and reduce its hydrophobicity. Our observations indicated that even low concentrations of $\mathrm{Ca}^{2+}$ can induce significant aggregation of $\mathrm{GO}$ at environmental relevant $\mathrm{pHs}$. Natural surface waters include both $\mathrm{Ca}^{2+}$ and $\mathrm{Mg}^{2+}$, but $\mathrm{Ca}^{2+}$ has the potential to exert a larger influence than $\mathrm{Mg}^{2+}$ due to its higher tendency to form 
complexes with carboxylated groups $[9,78]$. It is therefore expected that GO particles become unstable in natural waters when $\mathrm{Ca}^{2+}$ is present at or above a concentration of 0.5 $\mathrm{mM}[9]$.

\subsubsection{Impact of Natural Organic Matter}

Organic substances are abundant in natural waters, and they are chemically characterized by aromatic rings, carboxyl and hydroxyl groups. Humic acid (HA) and tannic acid (TA) were used as models of dissolved NOM to investigate the effects on $\zeta$ potential and hydrodynamic diameter of GO in the presence of electrolytes. Two different concentrations of HA and TA were used $(5,10) \mathrm{mg} / \mathrm{L}$, representative of the amount of NOM commonly found in ground and surface waters [166]. The compounds were selected in order to discriminate carboxyl related interactions from those associated with hydroxyl groups: HA contains various functional groups, $-\mathrm{COOH},-\mathrm{OH},-\mathrm{NH}_{2}$ [78], while TA is a polyphenolic compound, with a pKa of around 10. FTIR and XPS (Figure 4.2 (a), Figure 4.4 (b)) showed that the main functional groups of GO are epoxy and hydroxyl which are located on the basal of GO and carboxyl groups are in the edge. Thus, the possible interactions between HA, TA and GO could be via hydrogen bonds, Lewis acid-base, and $\pi-\pi$ interactions. Lewis acid-base interaction between HA and GO can occur due to proton complexation of the $\pi$ electron system of the graphene. Furthermore, - $\mathrm{COOH},-\mathrm{OH}$, and $\mathrm{NH}_{2}$ groups in HA can bind with epoxy, hydroxyl, and carboxyl groups in GO by strong hydrogen-bonding interaction [78].

The results showed the HA and TA had negligible effect on $\mathrm{GO} \zeta$-potential in presence of different salts, $\mathrm{NaCl}$ and $\mathrm{CaCl}_{2}$ (Figure 4.9). However, the aggregation of $\mathrm{GO}$ was 
proportionally reduced by adding $5 \mathrm{mg} / \mathrm{L}$ and $10 \mathrm{mg} / \mathrm{L}$ of humic acid respectively in presence of $\mathrm{NaCl}$ (Figure 4.10a), in agreement with observations by Chowdhury et al. [65]. Adding TA did not affect the aggregation of GO at $\mathrm{pH}$ between 5-11. In these cases, the stability of GO increases with increasing the concentration of NOMs because as $\mathrm{Ca}^{2+}$ can bridge to deprotonated carboxyl groups on organic matter [158], HA and TA compete with GO for binding cations, reducing the availability of free $\mathrm{Ca}^{2+}$ in solution. $\zeta$-potential measurements of $\mathrm{GO}$ in the existence of $\mathrm{HA}$ or TA in $1 \mathrm{mM}$ of each electrolyte did not show variations compared to those observed in the absence of organic matter. We can then conclude that dissolved NOM (most likely partially adsorbed on the GO particles) did not prevent $\mathrm{Ca}^{2+}$ interaction (specifically adsorption) with the surface, and no significant change in the magnitude of the repulsive electrostatic forces should be expected by the addition of NOM.

In fact, $\zeta$-potentials at $\mathrm{pH}=2$ for all conditions are within the experimental error. The two inflexion points at the carboxyl and hydroxyl pKa's were present in all $\mathrm{NaCl}$ suspensions, regardless of the added concentration of organic compounds. However, $\mathrm{Ca}^{2+}$ specific adsorption on the GO surface occurred under all tested conditions, as evidenced by the lack of $\zeta$-potential drop at higher pHs. 

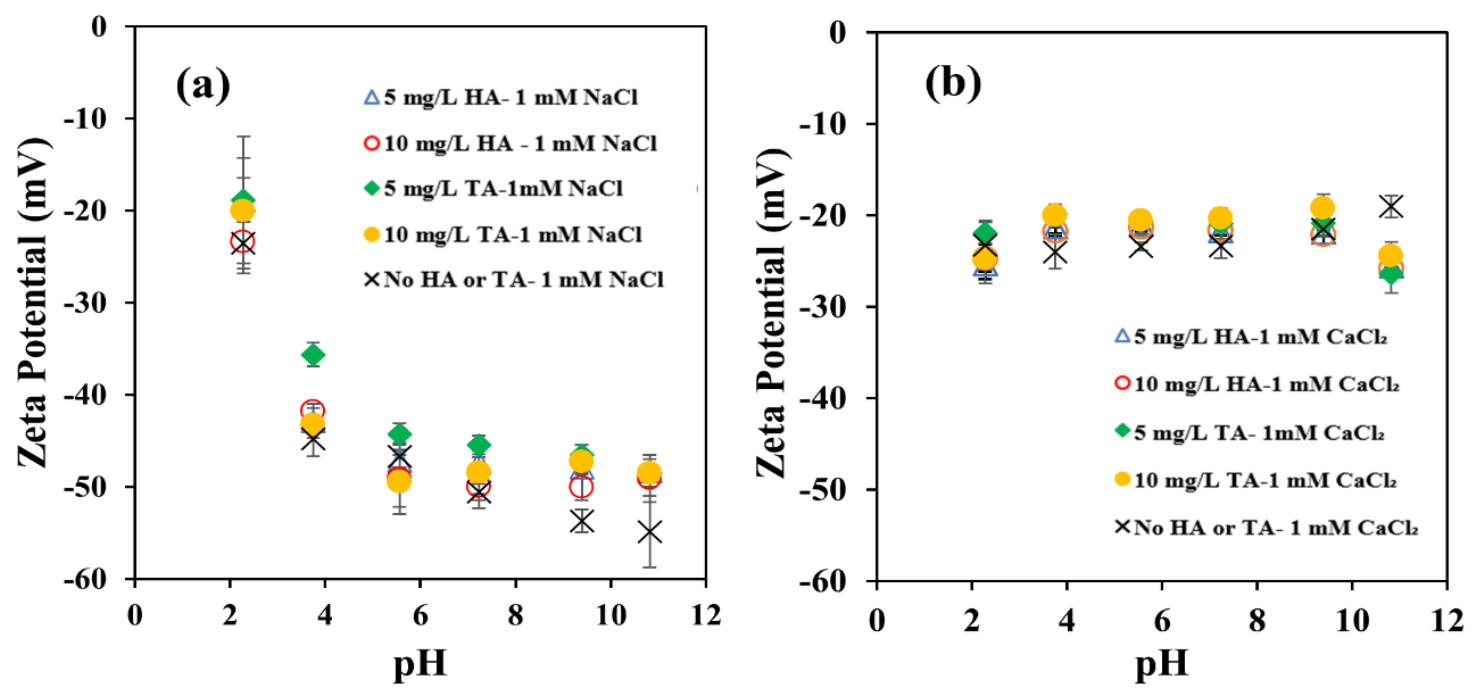

Figure 4.9 Zeta potential of GO as a function of $\mathrm{pH}$. (a) in presence of $5 \mathrm{ppm}, 10 \mathrm{ppm} \mathrm{HA}$ and TA respectively with $1 \mathrm{mM}$ of $\mathrm{NaCl}$. (b) in presence of $5 \mathrm{ppm}, 10 \mathrm{ppm}$ HA and TA respectively with $1 \mathrm{mM} \mathrm{CaCl}_{2}$.
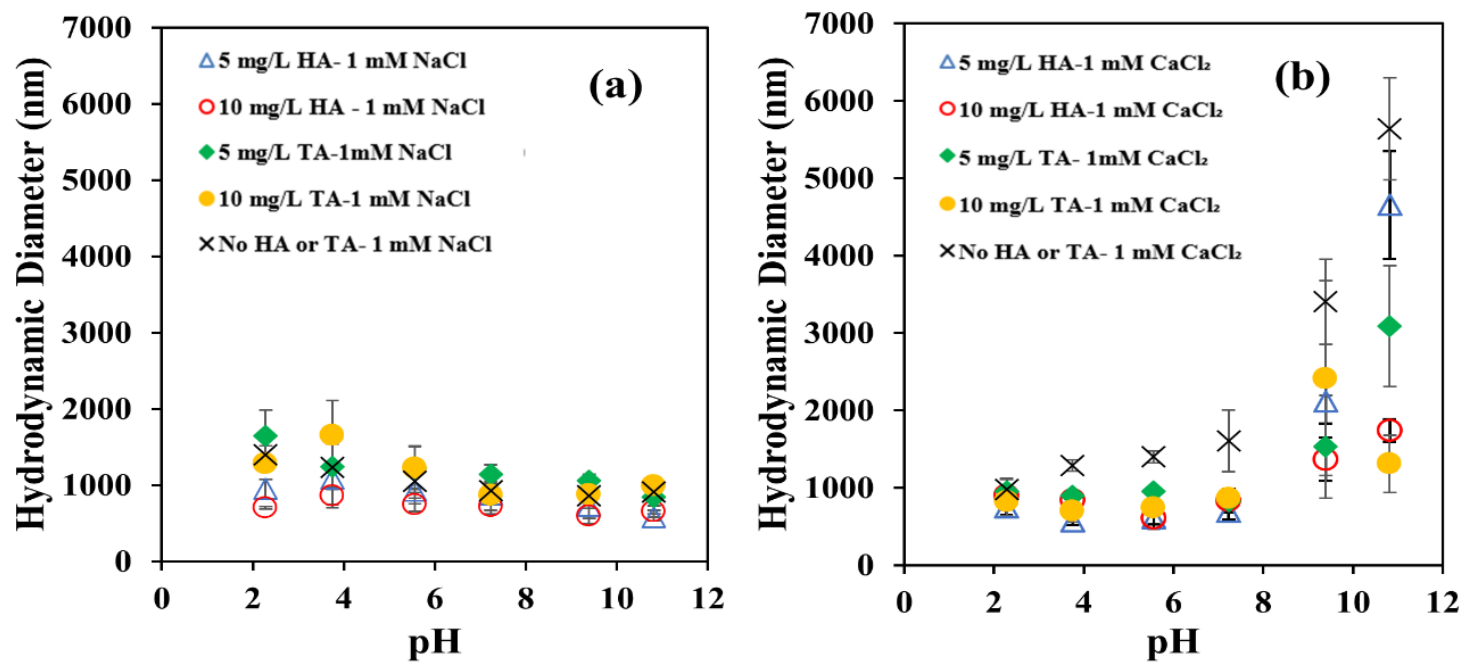

Figure 4.10 Hydrodynamic diameter of $\mathrm{GO}$ as a function of $\mathrm{pH}$. (a) in presence of $5 \mathrm{ppm}, 10$ ppm $\mathrm{HA}$ and TA respectively with $1 \mathrm{mM}$ of $\mathrm{NaCl}$. (b) in presence of $5 \mathrm{ppm}, 10 \mathrm{ppm}$ HA and TA respectively with $1 \mathrm{mM} \mathrm{CaCl}_{2}$.

The presence of organic matter did not preclude the adsorption of the divalent cation onto the particle surface; even if a layer of HA or TA formed on the GO, these compounds 
presented similar functionality as the bare surface, and therefore adsorption of $\mathrm{Ca}^{2}+$ was able to proceed. Analysis of $D_{h}$ of the GO particles as a function of $\mathrm{pH}$ suspended in $1 \mathrm{mM}$ $\mathrm{NaCl}$ and $\mathrm{NOM}$ showed negligible variation with respect to the situation in its absence; in all cases, a slight decrease in average size was observed as the $\mathrm{pH}$ turned more basic. Since $\zeta$-potentials were considerably low and similar in all cases, the stability can be attributed to electrostatic repulsion.

As we have already discussed, $\mathrm{Ca}^{2+}$ ions promoted aggregation for less acidic $\mathrm{pHs}$ as a consequence of surface complexation with multiple GO particles. However, this effect was inhibited in the presence of organic matter due to steric effects of HA and TA, which have molecular weight distributions of 170-22,600 Da and < 17,000 Da respectively [170]. HA and TA molecules adsorbed onto GO, binding via $\pi-\pi$ interactions and hydrogen bonds [171] and preventing close approximation of the GO nanosheets and increasing their stability [158]. Moreover, the stabilization effect was more noticeable at basic conditions. Inspection of the hydrodynamic diameter at the two highest $\mathrm{pH}$ levels ( 9 and11) revealed smaller aggregate size with increasing NOM concentration, TA being more effective at preventing aggregation than HA. As the NOM adsorbed on the GO particles, there was no modification of the chemical nature of the surface functionality $(\mathrm{OH}, \mathrm{COOH}$ mostly $)$, but their relative abundance was affected by the type of organic matter adsorbed. GO particles and HA had variable ratios of carboxylic:hydroxylic functional groups, while TA only provided $\mathrm{OH}$ functionality. As a result, TA adsorption on GO particles hindered access to $\mathrm{COOH}$ groups. Since $\mathrm{Ca}^{2+}$ can bridge deprotonated carboxyl groups on organic matter [167] and by a similar mechanism on GO particles, the presence of TA contributed to GO stabilization caused by the reduction of the number of $\mathrm{COOH}$ surface groups available. 
The central role of the chemical properties of the GO surface, in particular the types of surface groups, has been recognized [74][72]; the results presented here demonstrate that this influence extends to the characteristics of the dissolved NOM in natural waters.

\subsubsection{DLVO Modeling}

Classical DLVO theory has been widely used to predict the colloidal behavior of charged particles in suspension. The modeling equations accounts for two effects resulting from the addition of salts on repulsion: a decrease in $\zeta$-potential and an increase in Debye length $(\mathrm{K})$; both will decrease the repulsion at a given separation distance, and hence promote aggregation.

The applicability of the DLVO model as a predictive tool was investigated by modeling GO sheets in two different geometries: 3D sphere-like particles and 2D particles of finite thickness. In case of sphere-sphere model, the ratio of total interaction energies to thermal energy $k T$ were calculated as a function of separation distance. The influence of $\mathrm{pH}$, electrolyte concentration $\left(\mathrm{NaCl}\right.$ and $\mathrm{CaCl}_{2}$ ), and $\mathrm{NOM}$ (Figure 4.11, 4.12, 4.13) was investigated. High energy barrier and negligible secondary minimum were observed at the

lower $\mathrm{NaCl}$ and $\mathrm{CaCl}_{2}$ concentrations. Increasing IS led to a reduced energy barrier and aggregation, following a trend in good agreement with experimental observations (Figure 4.6). The height of the energy barrier was related to the size of particles. Pelley and Tufenkji [144] demonstrated that particle interaction energies are very sensitive to particle size changes. The peak of repulsive energy barrier and the depth of secondary energy well increased dramatically with increasing particle diameter. 
GO sheets were alternatively modeled as a thin plate with uniform surface charges. In this second approach, plate-plate interaction expressions were used and compared to the sphere-sphere case. Figure S8 shows the DLVO interaction energies calculated using the plate-plate model.

The modeling did not accurately predict the GO aggregation behavior observed experimentally, especially in the present of $\mathrm{Ca}^{2+}$ (Figure $4.14(\mathrm{~b}, \mathrm{~d})$ ). We hypothesize that the reason for this observation is that the aggregation of GO is not only an accumulative process by electrical double layer suppression but it is also accompanied by structural transformation of GO nanoparticles into 3D aggregates. Evidence to support this postulate can be found in the TEM images of aggregates formed in the presence of $\mathrm{Ca}^{2+}$ (Figure 4.1 (b)). The 2D GO can be transformed into a 3D sphere-like structure due to surface interaction with both heavy metals ions and common cations; therefore, GO aggregation can be correctly modeled using DLVO sphere-sphere interaction expressions, although they do not represent its true geometry in its non-aggregated state [157]. 

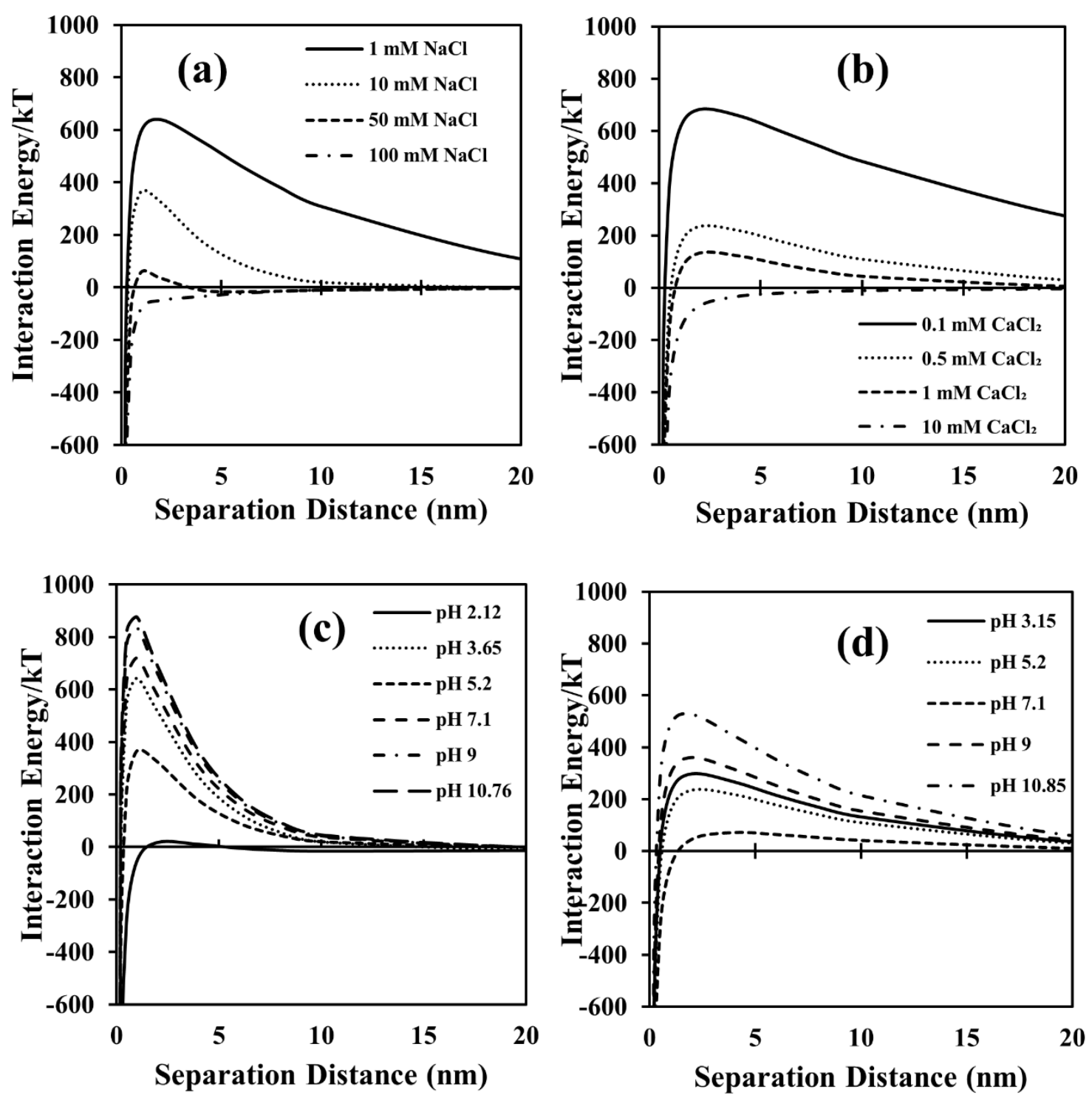

Figure 4.11 Sphere-sphere DLVO modeling; effect of ionic strength and $\mathrm{pH}$ variations. (a) GO with different $\mathrm{NaCl}$ concentrations. (b) $\mathrm{GO}$ with different $\mathrm{CaCl}_{2}$ concentrations. (c) $\mathrm{GO}$ in $10 \mathrm{mM}$ $\mathrm{NaCl}$ with different $\mathrm{pH}$. (d) $\mathrm{GO}$ in $0.5 \mathrm{mM} \mathrm{CaCl}_{2}$ with different $\mathrm{pH}$. 

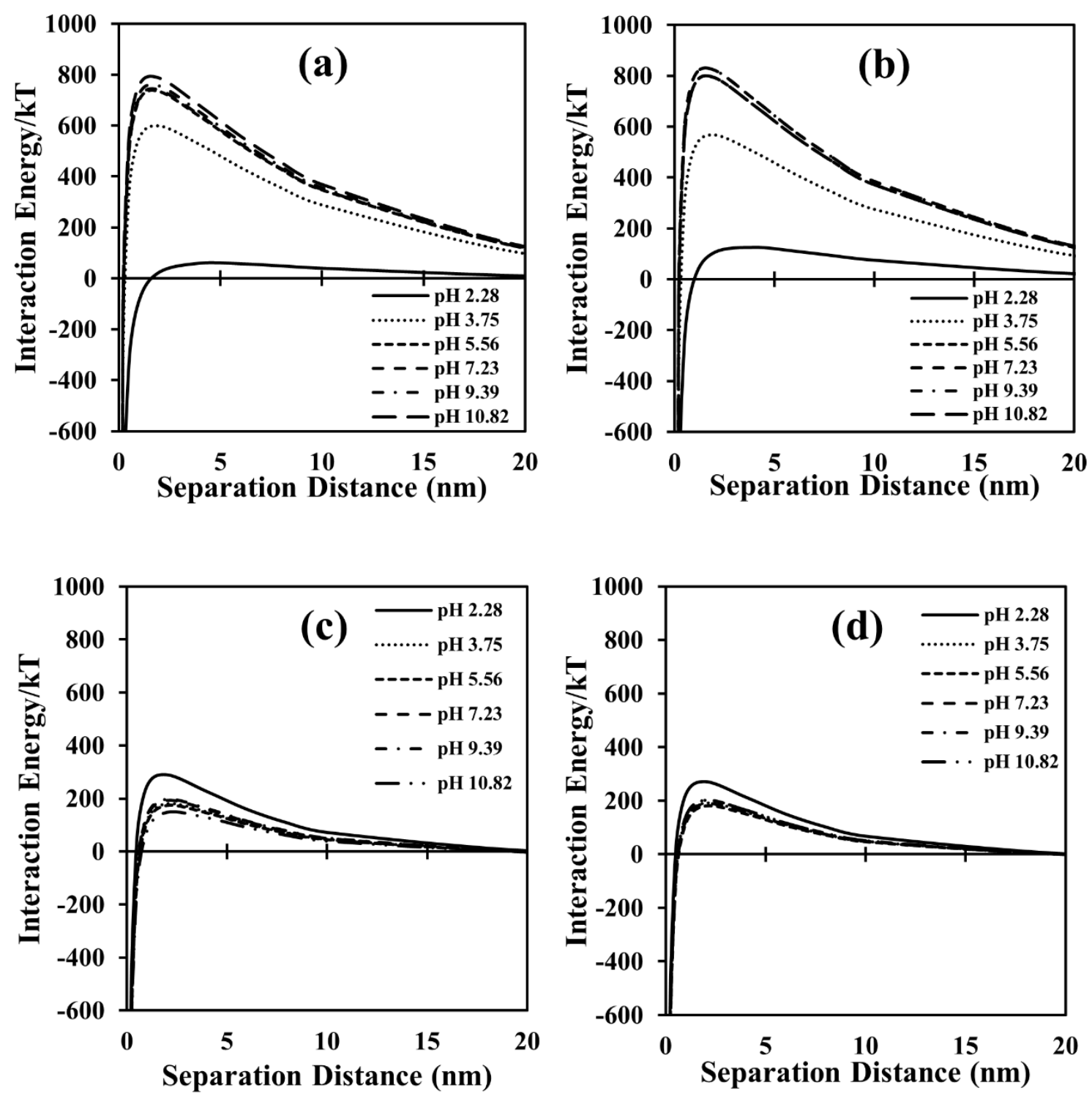

Figure 4.12 Sphere-sphere DLVO modeling; effect of humic acid and $\mathrm{pH}$ variations. (a) GO in 1 $\mathrm{mM} \mathrm{NaCl}$ with $5 \mathrm{mg} / \mathrm{L} \mathrm{HA}$. (b) GO in $1 \mathrm{mM} \mathrm{NaCl}$ with $10 \mathrm{mg} / \mathrm{L} \mathrm{HA}$ (c) $\mathrm{GO}$ in $1 \mathrm{mM} \mathrm{CaCl}_{2}$ with $5 \mathrm{mg} / \mathrm{L} \mathrm{HA}$. (d) GO in $1 \mathrm{mM} \mathrm{CaCl} 2$ with $10 \mathrm{mg} / \mathrm{L} \mathrm{HA}$. 

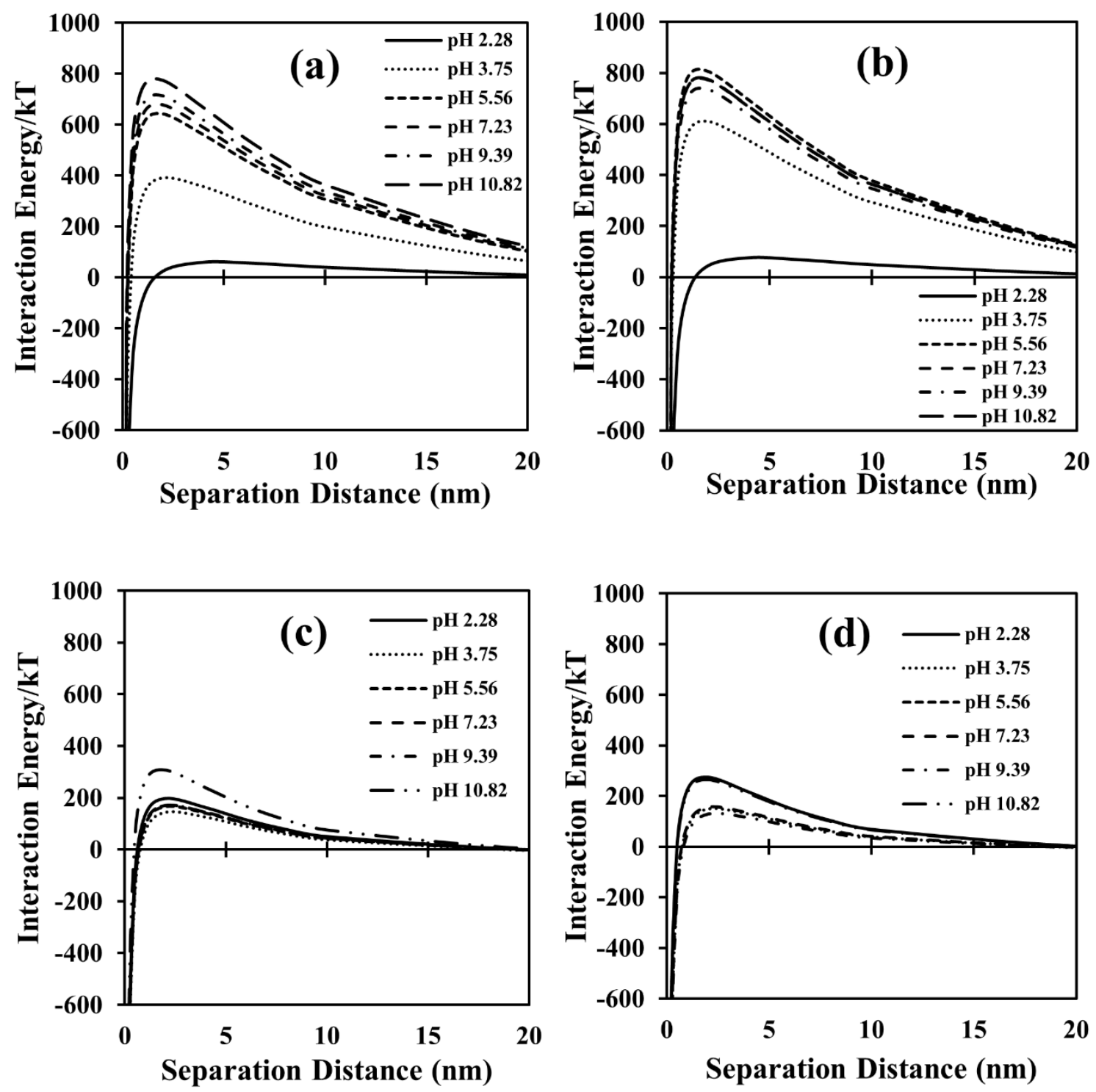

Figure 4.13 Sphere-sphere DLVO modeling; effect of tannic acid and pH variations. (a) GO in 1 $\mathrm{mM} \mathrm{NaCl}$ with $5 \mathrm{mg} / \mathrm{L} \mathrm{TA}$. (b) $\mathrm{GO}$ in $1 \mathrm{mM} \mathrm{NaCl}$ with $10 \mathrm{mg} / \mathrm{L} \mathrm{TA}$ (c) $\mathrm{GO}$ in $1 \mathrm{mM} \mathrm{CaCl}_{2}$ with $5 \mathrm{mg} / \mathrm{L} \mathrm{TA}$. (d) $\mathrm{GO}$ in $1 \mathrm{mM} \mathrm{CaCl} 2$ with $10 \mathrm{mg} / \mathrm{L}$ TA. 

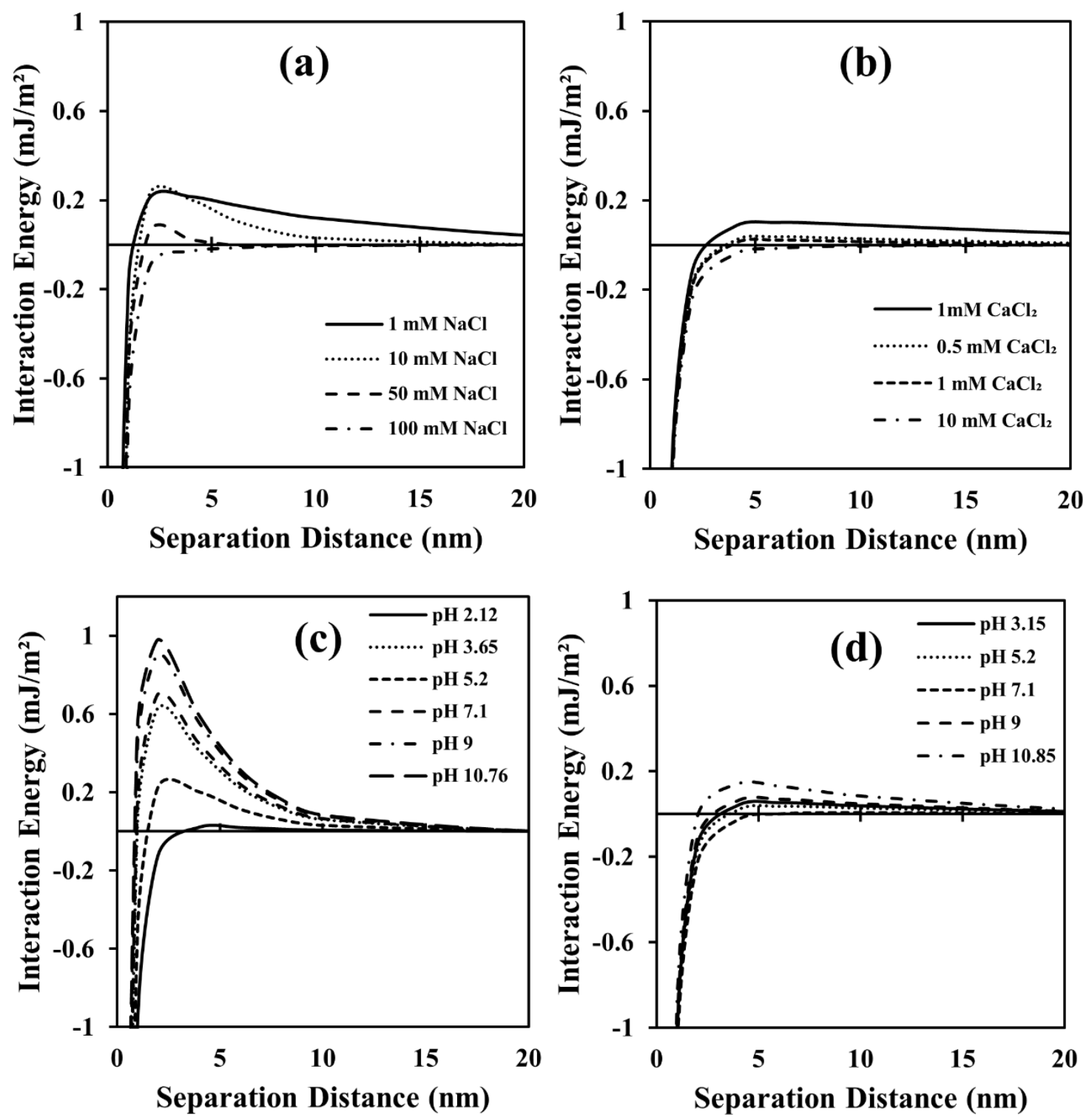

Figure 4.14 Plate-plate DLVO modeling; effect of ionic strength and $\mathrm{pH}$ variations. (a) GO with different $\mathrm{NaCl}$ concentrations. (b) $\mathrm{GO}$ with different $\mathrm{CaCl}_{2}$ concentrations. (c) $\mathrm{GO}$ in $10 \mathrm{mM}$ $\mathrm{NaCl}$ with different $\mathrm{pH}$. (d) $\mathrm{GO}$ in $0.5 \mathrm{mM} \mathrm{CaCl}_{2}$ with different $\mathrm{pH}$. 


\subsection{Conclusion}

Divalent cations $\left(\mathrm{Ca}^{2+}\right)$ affect $\mathrm{GO}$ stability more noticeable than monovalent cations $\left(\mathrm{Na}^{+}\right)$because of the binding capacity of $\mathrm{Ca}^{2+}$ ions with functional groups of GO. Presence of NOM did not prevent $\mathrm{Ca}^{2+}$ from specifically adsorb at the surface, and therefore, repulsive electrostatic forces in the presence of NOM were comparable to those when only the electrolytes are present. Moreover, divalent cations adsorption on the particle surface occurred under conditions of presence and absence of NOM, as the molecules present similar functionality to that of the clean surface, resulting in $\mathrm{Ca}^{2+}$ promoted aggregation. However, this effect depended on the chemical functionality of the organic molecule, in particular on its concentration of carboxylic groups. GO aggregation can be accurately modeled with DLVO sphere-sphere interaction expressions, suggesting that the behavior of the aggregated GO is dominated by the crumpled, 3D sphere-like structure that it assumes in water suspension as it interacts with other solution constituents such as common cations. The results of this study highlighted the predominant effect of specific interactions as well as the chemical structure of adsorbed organics on GO stability in natural. 


\title{
CHAPTER FIVE
}

\section{Laser Induced Graphene /Ceramic Membrane Composite: Preparation and Characterization}

\begin{abstract}
In this work, laser induced graphene (LIG) was successfully fabricated on microporous ceramic membranes. The surface area, morphology, and chemical characterizations were performed on the LIG layer. Water contact angle measurements showed the hydrophobicity of LIG. Pure water and solvents with different polarities were used to understand the solvent flux behavior of LIG membrane. The LIG membrane showed very high non-polar solvent fluxes and remarkably low water permeability, and thus, the transport through the LIG membrane is related to dipole moment and dielectric constant, represented by solvent polarity. The LIG membrane achieved $90 \%$ rejection for $255 \mathrm{~nm}$ diameter silica particles, suggesting the presence of submicron size connecting pore channels that dominate the transport mechanism.
\end{abstract}

Keywords: laser induced graphene; LIG/ceramic composite; solvent permeation 


\subsection{Introduction}

Membranes are a promising technology expected to be effective at reducing water contaminant separation costs and solving the water resource challenges faced by humanity as well as other environmental problems. Comparing with conventional separation methods such as distillation or adsorption, membrane separation is an environmentally friendly and energy-efficient technology, with a smaller footprint that can be operated in a continuous manner [172-175]. An ideal membrane provides selectivity, stability and high permeate flux and it is resistant to irreversible fouling. Pore size, morphology and surface chemical properties are key parameters for membrane design; specifically, a material that shows high affinity for the solvent (water) and repels the contaminants to be separated is desired. Surface charge, hydrophobicity/hydrophilicity of the membrane, and pores size distribution are then main parameters in assessing membrane performance [5].

Among the membrane community, graphene nanomaterials have generated immerse interest in water purification application [176-178] and have opened the door for assembling membranes with improved separation capabilities [179]. Graphene, a singleatom-thick sheet of $\mathrm{sp}^{2}$ hybridized carbon atoms, was first fabricated in 2004 [6]. With its persistent properties, graphene has displayed great potential for atomic permeation, water transport, and gas separation and attracted significant attention in diverse branches of science and technology [9-13].

The concept and the preparation of graphene-based materials have been explored for over 100 years. The use of strong oxidizing mixtures, such as nitric acid $\left(\mathrm{HNO}_{3}\right)$ and potassium chlorate $\left(\mathrm{KClO}_{3}\right)$ was reported as early as mid-1800s [184]. In 1958, 
Hummers reported a method to fabricate graphitic oxide [18], which is still the basis for current fabrication method of graphene oxide nanomaterials. However, these methods produced toxic gases $\left(\mathrm{ClO}_{2}, \mathrm{NO}_{2}\right.$ and $\left.\mathrm{N}_{2} \mathrm{O}_{4}\right)$, and other hazardous chemicals (potassium permanganate, sulfuric acid, and sodium nitrate). These flaws led researchers to search for greener synthesis methods. For example, Chen et al. excluded $\mathrm{NaNO}_{3}$ from the reaction, which resulted in lower amounts of waste generated [19]. Marcano et al. also modified Hummers' method to exclude $\mathrm{NaNO}_{3}$, but more $\mathrm{KMnO}_{4}$ was required [20]. For various applications, graphene is engineered into three-dimensional (3D) porous structures to provide high surface area to preserve its high mobility and mechanical stability $[100,102]$. Conventional procedures for the fabrication of 3D graphene include the assembly of graphene oxide (GO) into a foam. However, this procedure needs the GO precursor to be prepared through its oxidative, hazardous synthesis route $[99,100]$. Chemical vapor deposition (CVD) on porous substrates also produces 3D graphene, but the high temperature conditions and subsequent etching and drying process may hinder its scalable production [102,103].

Laser induced graphene (LIG), first reported by Lin et al. [21], can be fabricated in one-step, scalable, reagent-free process, by irradiation of a commercial polyimide (PI film) by a $\mathrm{CO}_{2}$ infrared laser under ambient conditions. LIG has been studied for supercapacitors and energy storing devices [185] and recently in environmental applications, such as coating material for low fouling surfaces, antimicrobial electrodes [23] and adsorbents [24]. The physicochemical characteristics of graphene, coupled with the ease and flexibility of the LIG fabrication method, make it a promising route for the 
fabrication of highly hydrophobic membranes specially designed for solvent separation or membrane distillation processes.

In present work, we successfully fabricated for the first time an asymmetric composite ceramic membrane, creating a LIG graphene coating as an effective separation layer on top of a porous ceramic support. The LIG was characterized as well as the parameters in the laser conversion process (laser power and raster speed). The membrane permeability was investigated at operation pressure between $69 \mathrm{kPa}$ and $138 \mathrm{kPa}$. Different chemicals were used to study the effect of solvent properties (surface tension, molar volume, H-bonding contribution of the Hansen solubility parameter, dipole moment, dielectric constant, and viscosity) on transport through the membrane.

\subsection{Materials and methods}

\subsubsection{Fabrication of laser induced graphene (LIG) on microfiltration ceramic membrane}

An LIG layer was fabricated on titanium dioxide $\left(\mathrm{TiO}_{2}\right)$ microfiltration ceramic discs with a diameter $47 \mathrm{~mm}$, nominal pore size $1.4 \mu \mathrm{m}$, and thickness $2.6 \pm 0.02 \mathrm{~mm}$ (Sterlitech, Kent, WA, USA). The general procedure is presented in Figure 5.1. A $12.0 \mathrm{wt}$ $\%$ solution of poly(pyromellitic dianhydride-co-4,4-oxidianiline, amic acid) (PAA) (Sigma-Aldrich, St. Louis, MO, USA) was used in the initial coatings. The ceramic supports were wet with water before polymer coating in order to prevent the polymer solution from penetrating into the pores when the support pore volume was mostly occupied by water. To obtain a liquid layer of uniform thickness, $2 \mathrm{ml}$ of PAA solution 
were placed on the support surface and it was spun using a spin coater (Laurell WS-650 MZ-23NPPB, North Wales, PA, USA) at 3000 r.p.m for 30 seconds. Then, PAA was converted to polyimide (PI) by thermal imidization, heating the sample at a rate of $10{ }^{\circ} \mathrm{C}$ $\mathrm{min}^{-1}$, from room temperature to $300{ }^{\circ} \mathrm{C}$ in three steps (up to $100{ }^{\circ} \mathrm{C}, 200{ }^{\circ} \mathrm{C}$ and $300{ }^{\circ} \mathrm{C}$ ), and holding the temperature for 60 minutes at each temperature. The PI was irradiated using a computer-controlled $\mathrm{H}$-series Desktop $\mathrm{CO}_{2}$ laser to photothermally convert the $\mathrm{sp}^{3}$-carbon atoms to $\mathrm{sp}^{2}$-carbon atoms. The laser operation conditions were: power of 10 $\mathrm{W}$, raster speed of $0.61 \mathrm{~m} \mathrm{~s}^{-1}\left(24\right.$ inch s$\left.^{-1}\right)$, and image density of 1000 pulses inch $^{-1}$ in both axes. Figure 5.2 shows digital images of the ceramic membrane supports before and after coating with PI and LIG.

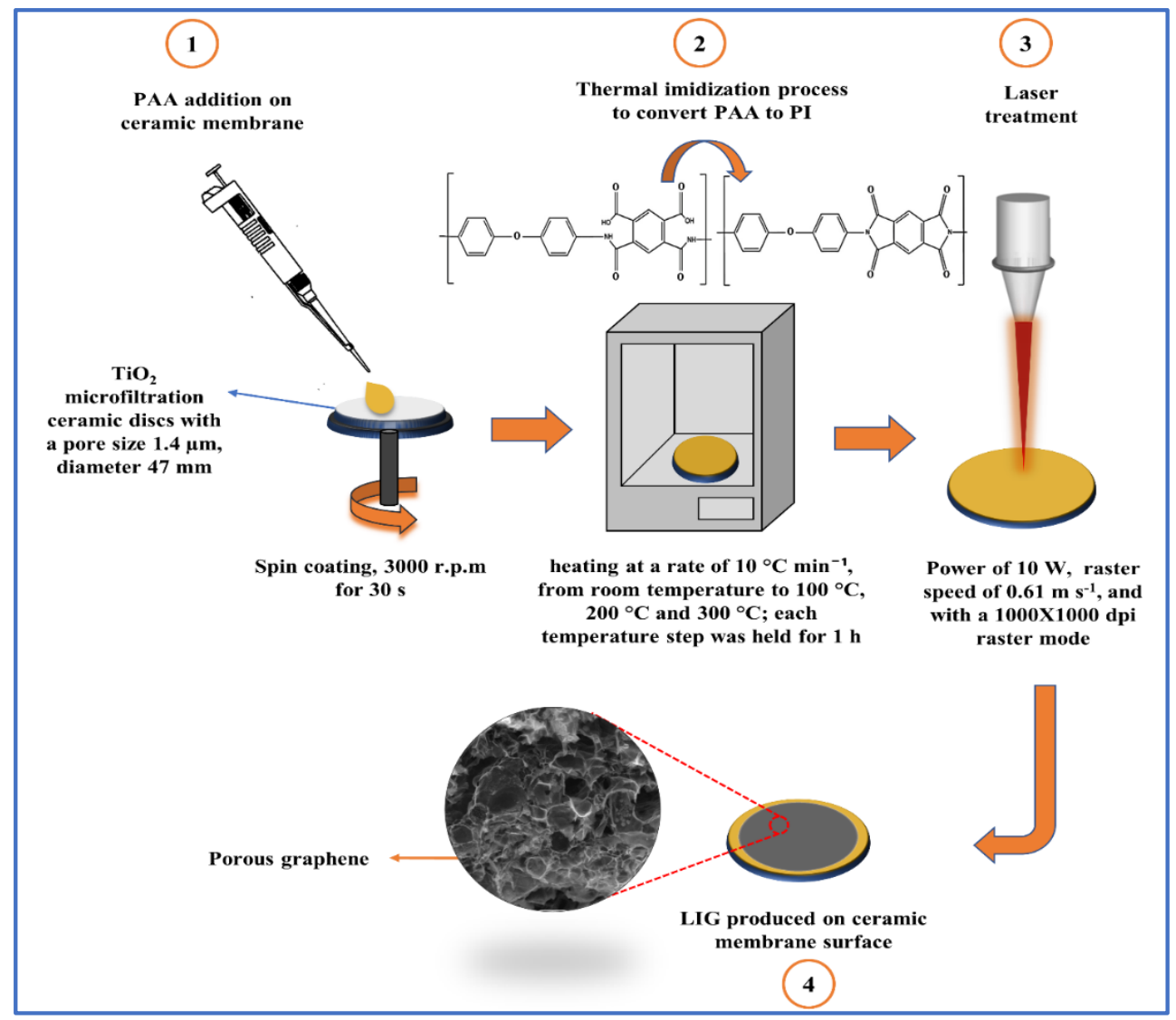

Figure 5.1 General procedure for the preparation of laser induced graphene/ceramic membrane (LIG/CM) composite. 


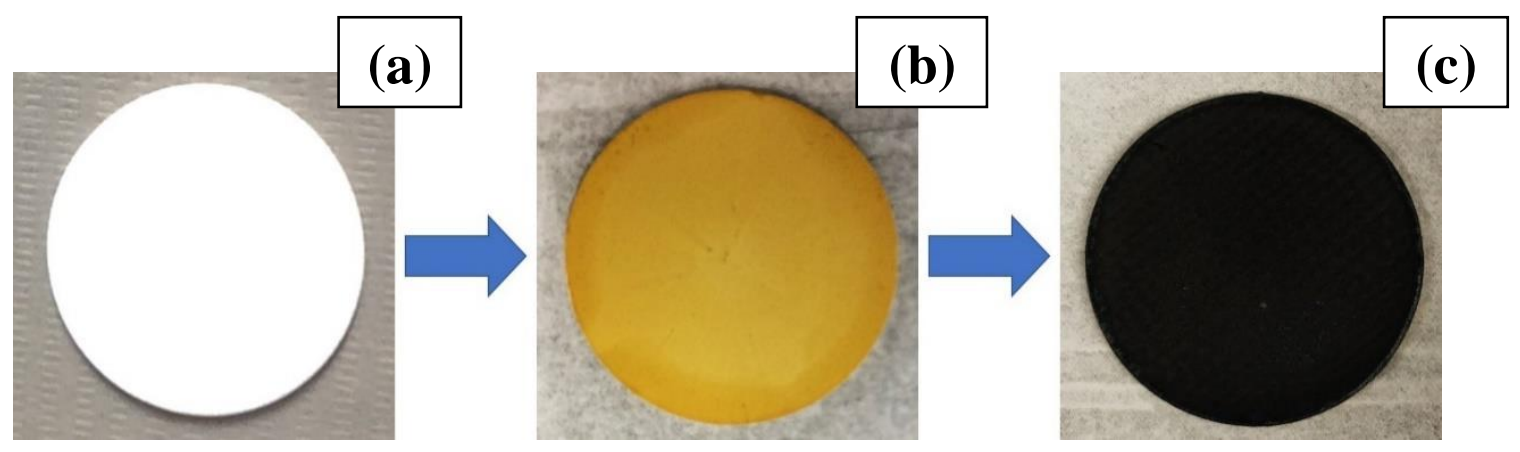

Figure 5.2 Digital images of (a) Ceramic membrane before coating. (b) Ceramic membrane after coating with PI. (c) Ceramic membrane after laser treatment of PI.

\subsubsection{Characterization techniques}

The morphology and the thickness of PI and LIG were observed using a FEI Quanta 600 FEG environmental scanning electron microscope (ESEM) (ThermoFisher Scientific, Hillsboro, OR, USA); a Tecnai F30 twin transmission electron microscope (TEM) (ThermoFisher Scientific, Hillsboro, OR, USA) was used to obtain high-resolution images for the nano structure of LIG flakes and Brunauer-Emmett-Teller (BET) specific surface area was measured in a nitrogen adsorption surface area analyzer (SA 3100, Beckman Coulter, USA).

Quantitative surface roughness and morphology of the membrane were analyzed by optical profilometry (Wyko NT9100-Veeco, NY, USA). Average roughness $\left(\mathrm{R}_{\mathrm{a}}\right)$ was calculated as shown in Equation 5.1.

$$
R_{a}=\frac{1}{n} \sum_{i=1}^{n}\left|Z_{i}-\bar{Z}\right|
$$

where $\mathrm{n}$ is the number of measurements, $Z_{i}$ is the measured height, and $\bar{Z}$ is the mean height of the profile peak. 
The chemical functional groups were investigated by Fourier-transform infrared spectroscopy (FTIR), in a Nicolet 4700 (ThermoFisher Scientific, MA, USA) spectrophotometer using DRIFTS technique and $\mathrm{KBr}$ as transparent matrix. A commercially available PI film was used as reference material for PI (Kapton® Polyimide Film, thickness $127 \mu \mathrm{m}$, McMaster-Carr, USA). X-ray diffraction analysis (XRD) was conducted on PANanlytical XPert Pro, Netherlands with $\mathrm{Cu} \mathrm{K} \alpha$ radiation $(\lambda=1.54 \AA)$. The samples were scanned from $10^{\circ}$ to $50^{\circ}(2 \theta)$ with a step size of 0.025 and account time of $1 \mathrm{~s}$ at each point. Furthermore, the crystalline sizes along c axis (Lc) and domain size in the a axis (La) were calculated from XRD peaks by using Equation 5.2 and Equation 5.3 respectively [21]:

$$
\begin{aligned}
& L c=\frac{0.89 \lambda}{B_{1 / 2}(2 \theta) \operatorname{Cos} \theta} \\
& L a=\frac{1.84 \lambda}{B_{1 / 2}(2 \theta) \operatorname{Cos} \theta}
\end{aligned}
$$

where $\lambda$ is the $\mathrm{X}$-ray wavelength $\left(\lambda=1.54 \AA\right.$ ) and $B_{1 / 2}(2 \theta)$ (in radian units) is the full width at half maximum of the peaks.

X-ray photoelectron spectroscopy (XPS) was performed in an ultrahigh vacuum (Kratos Axis 165, Manchester, UK) using a monochromatic Al Ka source (hv = 1486.6 $\mathrm{eV}$, resolution $=0.5 \mathrm{eV})$. Raman microscope $($ Renishaw inVia, Gloucestershire, $\mathrm{UK})$ using laser power of $5 \mathrm{~mW}$ with a 633-nm laser excitation at room temperature was employed to acquire Raman spectra for LIG. A video contact angle measurement system (VCA-2500 XE, AST products, Billerica, MA, USA) was used to evaluate the 
hydrophobicity of LIG by measuring the pure water contact angles. At least six stabilized contact angles from different sites of each sample were obtained to calculate the average contact angle and standard deviation.

\subsubsection{Permeability and transport model}

Permeability tests were performed in dead-end stainless-steel filtration cell (Sterlitech stirred cell HP4750, Kent, WA, USA) at operating pressure of $69 \mathrm{kPa}, 103.4 \mathrm{kPa}$, and $138 \mathrm{kPa}$ (Figure 5.3). The cell was filled with the feed and pressurized with compressed air. Clean water flux $\left(\mathrm{J}\right.$ in $\left.\mathrm{L} \mathrm{m}^{-2} \mathrm{~h}^{-1}\right)$ was obtained by recording the volume of permeate collected in a graduated cylinder as a function of time. The effective filtration area was $1.13 \times 10^{-3} \mathrm{~m}^{2}$ for all samples. Permeate flux was calculated using Equation (5.4):

$$
J=\frac{V_{p}}{t . A}
$$

where $J$ is the permeate flux $\left(\mathrm{L} \mathrm{m}^{-2} \mathrm{~h}^{-1}\right), V_{p}$ is volume of the permeate $(\mathrm{L}), A$ is the filtration area $\left(\mathrm{m}^{2}\right), t$ is collecting permeate time (h). Permeability tests were conducted with different liquids: isopropanol (99.9\% purity), toluene (99.9\%), hexane (95\%).

Solvents were obtained from Sigma-Aldrich, St. Louis, MO, USA, and ultrapure water (18.2 $\mathrm{m} \Omega . \mathrm{cm}$ at $25^{\circ} \mathrm{C}$ ) from a Thermo Scientific ${ }^{\mathrm{TM}}$ Barnstead $^{\mathrm{TM}}$ E-Pure ${ }^{\mathrm{TM}}$ Ultrapure Water Purification System (Waltham, MA, USA).

Permeability was calculated using Darcy's equation:

$$
J=k \frac{\Delta P}{\mu}
$$


where $J$ is the permeate flux $\left(\mathrm{L} \mathrm{m}^{-2} \mathrm{~h}^{-1}\right), \Delta P$ the trans-membrane pressure, $\mu$ the fluid viscosity, $k$ the membrane permeability constant.

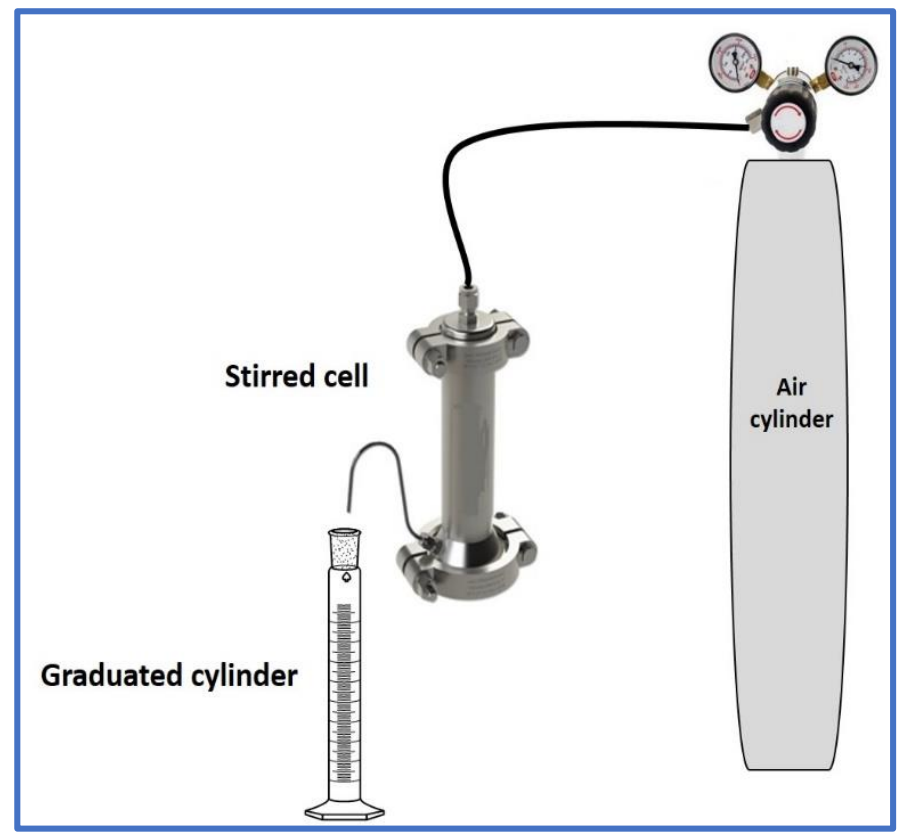

Figure 5.3 Permeability experimental set up.

LIG-solvent interactions are expected to vary due to the different properties of the liquids tested; for example, molecular volume, dielectric constant, viscosity, and polarity [186]. In order to examine the solvent flux through the laser induced graphene /ceramic membrane (LIG/CM), feed solutions were selected in order to display a range of such properties, as shown in Table 5.1. 
Table 5.1 Physical properties of the solvents used for permeability tests [187-190]

\begin{tabular}{|c|c|c|c|c|c|c|c|}
\hline Name & $\begin{array}{l}\text { Viscosity } \\
\mu(\mathrm{mPa} \mathrm{s} \\
\left.\text { at } 20^{\circ} \mathrm{C}\right)\end{array}$ & $\begin{array}{c}\text { Dipole } \\
\text { moment } \\
\text { (D) }\end{array}$ & $\begin{array}{c}\text { Dielectric } \\
\text { constant } \\
\text { (E) }\end{array}$ & $\begin{array}{c}\text { Surface } \\
\text { tension } \gamma \\
\left(\mathrm{mN} \mathrm{m}^{-1} \text { at }\right. \\
\left.20^{\circ} \mathrm{C}\right)\end{array}$ & $\begin{array}{c}\text { H-bonding } \\
\text { contribution of } \\
\text { Hansen solubility } \\
\text { parameter } \delta_{\mathrm{h}} \\
\left(\mathrm{Mpa}^{0.5}\right)\end{array}$ & $\begin{array}{c}\text { Molar } \\
\text { volume } \\
\left(\mathrm{cm}^{3}\right. \\
\left.\mathrm{mol}^{-1}\right)\end{array}$ & $\begin{array}{c}\text { Vapor } \\
\text { pressure } \\
\text { (mmHg at } \\
20)\end{array}$ \\
\hline n-Hexane & 0.31 & 0.08 & 1.89 & 18 & 0 & 130.6 & 129.1 \\
\hline Toluene & 0.59 & 0.34 & 2.40 & 30 & 2 & 105.9 & 26.3 \\
\hline Isopropanol & 2.39 & 1.66 & 19.92 & 21 & 16.4 & 76.9 & 78.2 \\
\hline Water & 1.00 & 1.87 & 78.54 & 72.8 & 42.3 & 18.0 & 17.5 \\
\hline
\end{tabular}

\subsubsection{Rejection measurements}

The separation properties of the prepared membranes were examined using variable size silica particles to conduct rejection experiments. Commercially available silica nanoparticles ST-O, ST-30-LH, and ST-ZL were obtained from Nissan Chemical, Houston, TX, with average size of $18.7 \pm 1.1 \mathrm{~nm}, 52.7 \pm 3.1 \mathrm{~nm}$, and $107 \pm 5.4 \mathrm{~nm}$ respectively. Additionally, silica particles (SP) synthesized in the laboratory following a modified Stöber method [191] were also tested, with average size of $255.6 \pm 9.5 \mathrm{~nm}$. In the rejection experiments, particles were suspended in methanol with concentrations between 100-200 $\mathrm{mg} \mathrm{L}^{-1}$. Filtrations were carried out at a transmembrane pressure of 138 $\mathrm{kPa}$ and temperature of $20^{\circ} \mathrm{C}$. At least triplicate experiments were completed. A ZetaSizer Nano ZS (Malvern Instruments, Worcestershire, U.K.) was used to measure hydrodynamic diameter $\left(D_{h}\right)$ as well as the concentration by measuring the derived count rate (kcps) [192,193]. Figure 5.1s (Supporting information) shows the calibration curves for the measurement of the silica particle concentrations used in this study. 


\subsection{Results and discussion}

\subsubsection{Fabrication of asymmetric membranes}

The ceramic supports were initially coated with the precursor polymer PAA, which was later thermally converted to PI by imidization. This process produced cyclization of the PAA chain and converted the spun coated layer into a rigid structure [194]. The conversion was investigated by FTIR; spectra was collected from Kapton film and PAA sheets cast on glass slides and then peeled to obtain free standing samples. The imidization process was conducted following the same experimental protocol for PAA deposited in glass slides and ceramic surfaces.

The FTIR spectra (Figure 5.4 (a)) of the converted PI revealed absorption bands at 1777 $\mathrm{cm}^{-1}$ ( $\mathrm{C}=\mathrm{O}$ asymmetric stretching of imide), $1725 \mathrm{~cm}^{-1}$ ( $\mathrm{C}=\mathrm{O}$ symmetric stretching), 1374 $\mathrm{cm}^{-1}$ (C-N stretching) and $723 \mathrm{~cm}^{-1}(\mathrm{C}=\mathrm{O}$ bending of imide), indicating the presence of the imide group and the completion of imidization process[195,196]. When compared to the FTIR spectrum of the commercially available Kapton film, similar features were identified, confirming the transformation of the PAA.

Chemical, structural and morphological analysis were conducted on a homogeneous, free standing LIG sample, prepared by laser treatment of Kapton films and scraping the formed graphene from the surface. The XRD results showed a strong peak at $2 \theta=25.9^{\circ}$, from which an interlayer spacing of 3.4 A between (002) planes in the LIG was obtained, confirming the crystalline structure of graphene. A second peak at $2 \theta=42.9^{\circ}$ indicated (100) reflections which are associated with an in-plane structure [21] (Figure 5.4(b)). The 
crystalline sizes along c axis (Lc) and a axis (La) were calculated to be $6.6 \mathrm{~nm}$ and 14.8 $\mathrm{nm}$ respectively.
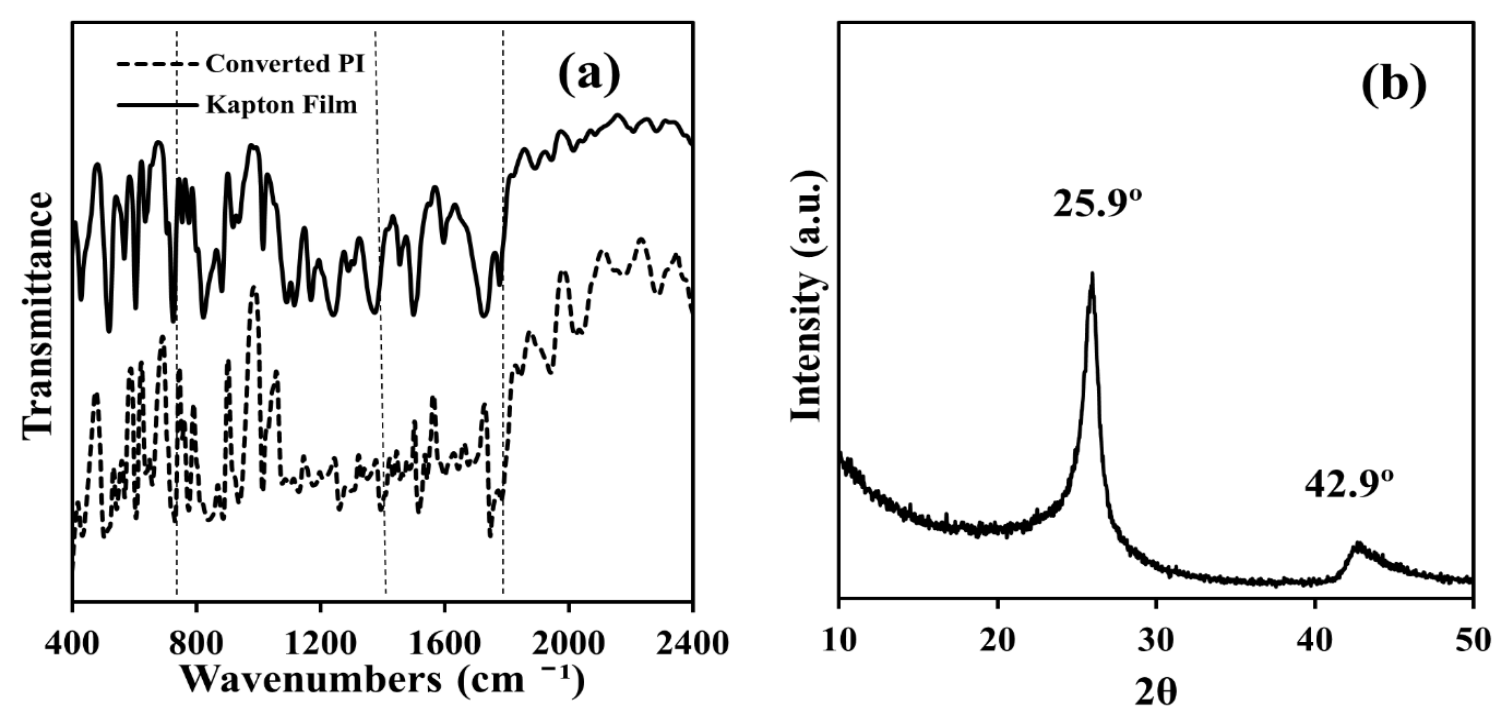

Figure 5.4 (a) FTIR spectroscopy of Kapton film and PI sheet. (b) XRD patterns of laser treated Kapton film.

The Raman spectrum of LIG on the ceramic support (Figure 5.5 (a)) showed three prominent peaks; the D peak at $\sim 1,350 \mathrm{~cm}^{-1}$ induced by defects $\mathrm{sp}^{2}$-carbon bonds, the $\mathrm{G}$ peak at $\sim 1,582 \mathrm{~cm}^{-1}$ that indicated in-plane vibrational mode which involves sp ${ }^{2}$ hybridized carbon atoms that comprises graphene sheets, and the $2 \mathrm{D}$ peak at $\sim 2,700 \mathrm{~cm}^{-1}$ that originated from second order zone-boundary phonons [21,197]. XPS elemental composition analysis showed that the oxygen percentage of LIG was $1.26 \pm 0.2 \%$, nitrogen percentage $0.88 \pm 0.02 \%$, and carbon percentage $97.86 \pm 0.3 \%$ (Figure. $5.5(\mathrm{~b})$ ). 

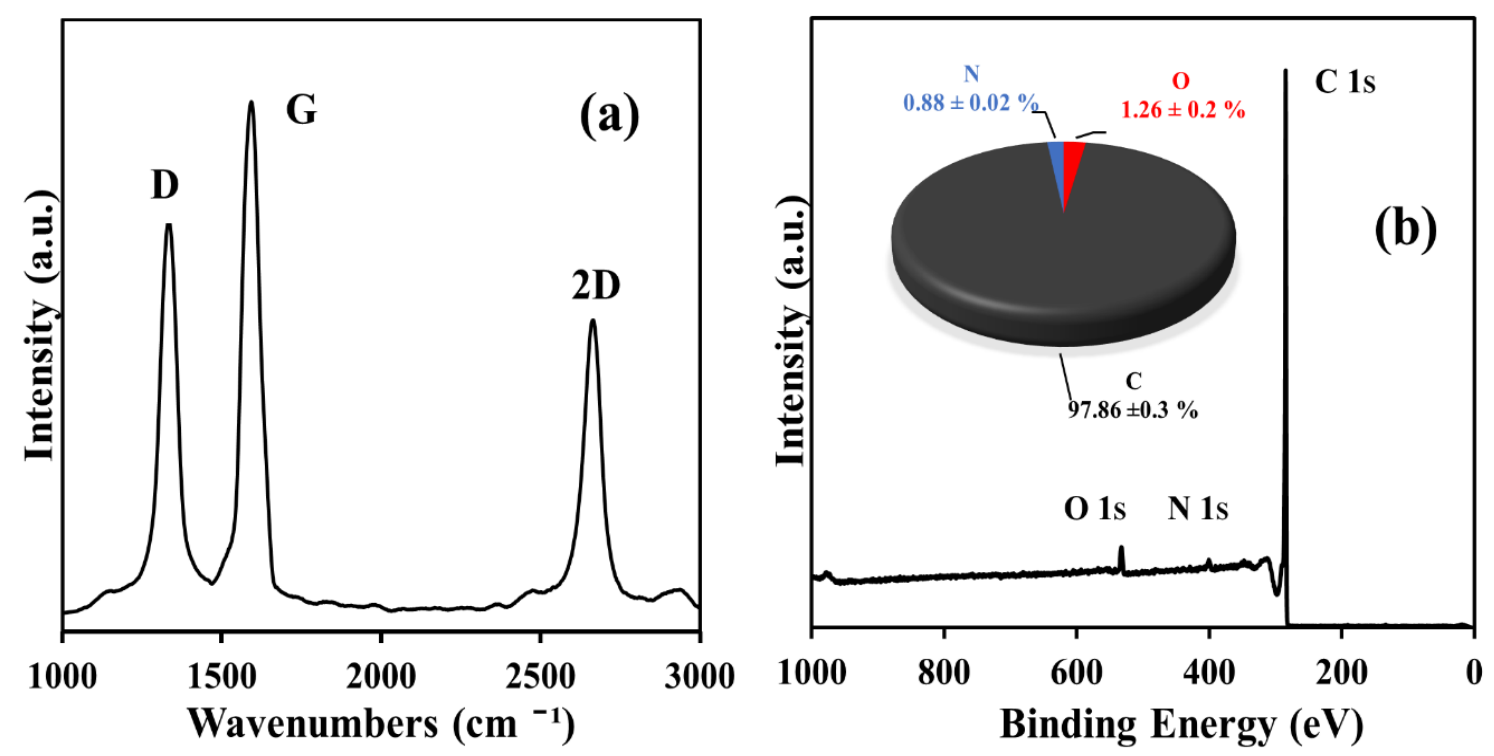

Figure 5.5 (a) Raman spectra of LIG on the ceramic membrane. (b) XPS spectra of LIG.

Figure 5.6 (a) shows a type II $\mathrm{N}_{2}$ adsorption isotherm displayed by LIG [198]. The mesopore size distribution curve was obtained using Barrett-Joyner-Halenda (BJH) method (Figure $5.6(\mathrm{~b})$ ). The average pore diameter and the specific surface area of LIG was $54.55 \mathrm{~nm}$ and $135 \pm 3.7 \mathrm{~m}^{2} \mathrm{~g}^{-1}$, respectively.

The measured water contact angle was $122^{\circ} \pm 5.4^{\circ}$ (Figure 5.7) which confirmed the hydrophobic nature of the graphene. Figure 5.8 depicts the changes in surface morphology that the membrane surface undergoes when coated with PI and after conversion to LIG. 

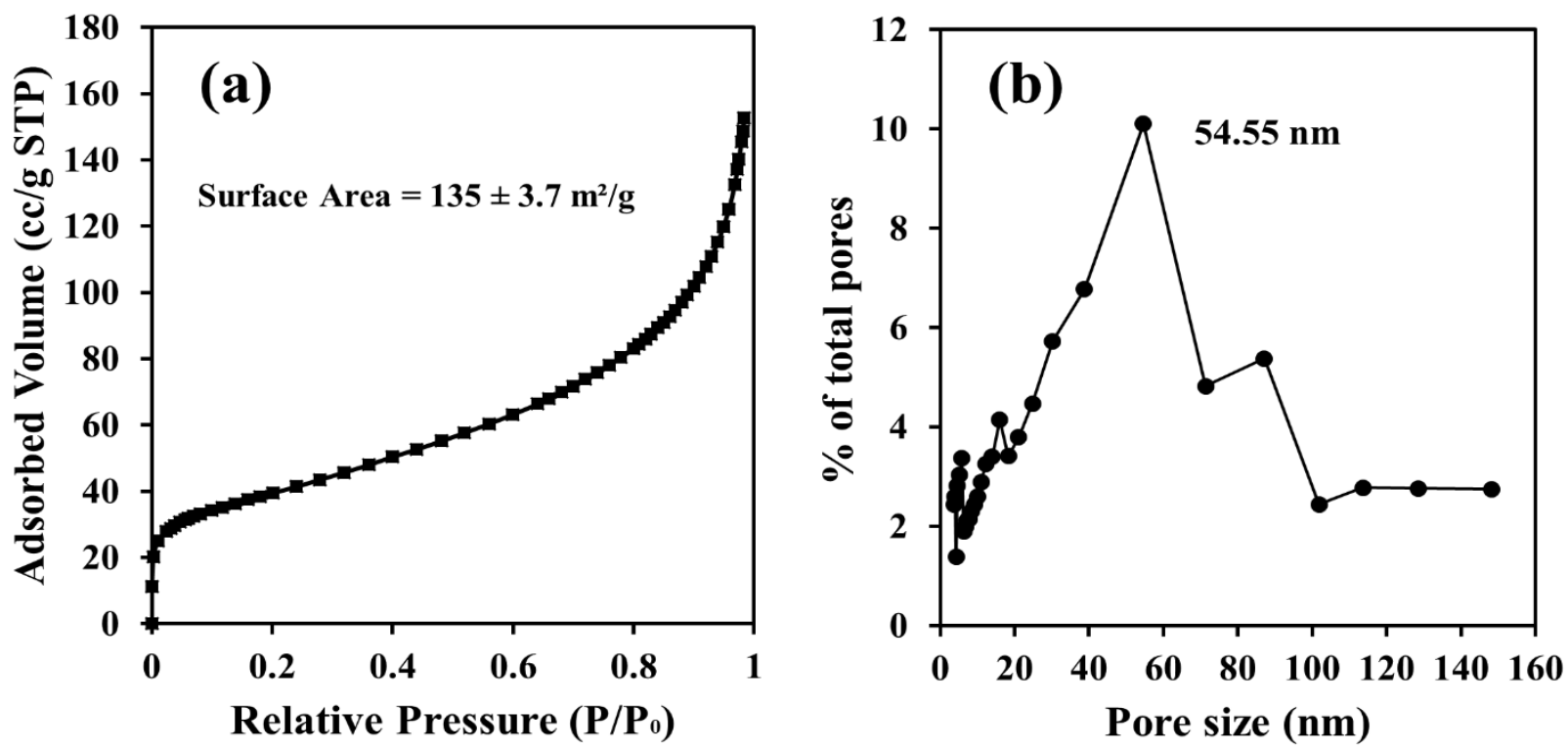

Figure 5.6 BET surface area measurement of LIG. (a) $\mathrm{N}_{2}$ adsorption isotherm. (b) pore size distribution.

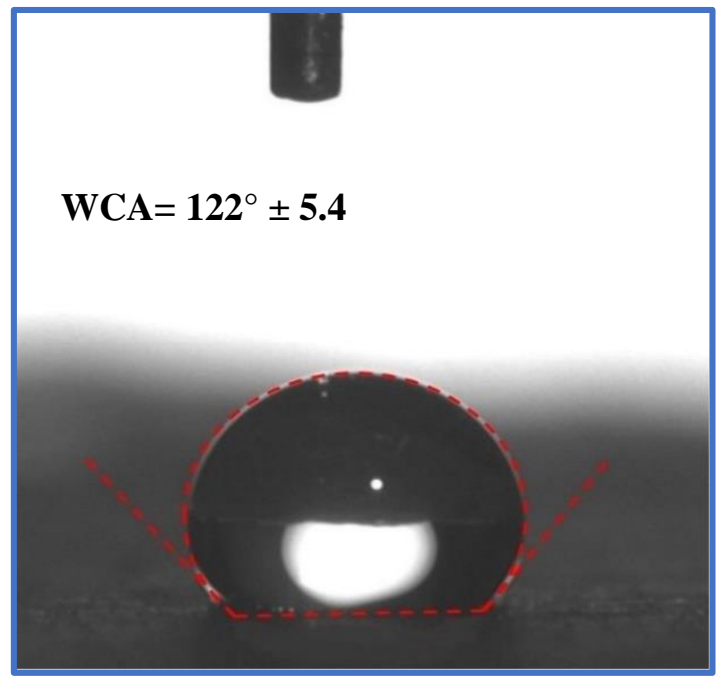

Figure 5.7 Water contact angles (WCA) with a $5 \mu \mathrm{L}$ water droplet of LIG on ceramic membrane.

In addition to imaging, optical profilometry was applied in order to quantitatively determine surface roughness. The average surface roughness decreased approximately 
$60 \%$ after PI coating but increased to more than $300 \%$ of the initial value when converted to LIG (Figure $5.8(\mathrm{~d})$ ). This substantial increase in surface roughness is due to the change of PI morphology as it converts to graphene by the laser treatment. To select the optimum parameters which ensure the conversion of PI to LIG, different laser power levels $(8 \mathrm{~W}-13 \mathrm{~W})$ and raster speeds $\left(0.4 \mathrm{~m} \mathrm{~s}^{-1}-0.8 \mathrm{~m} \mathrm{~s}^{-1}\right)$ were investigated.
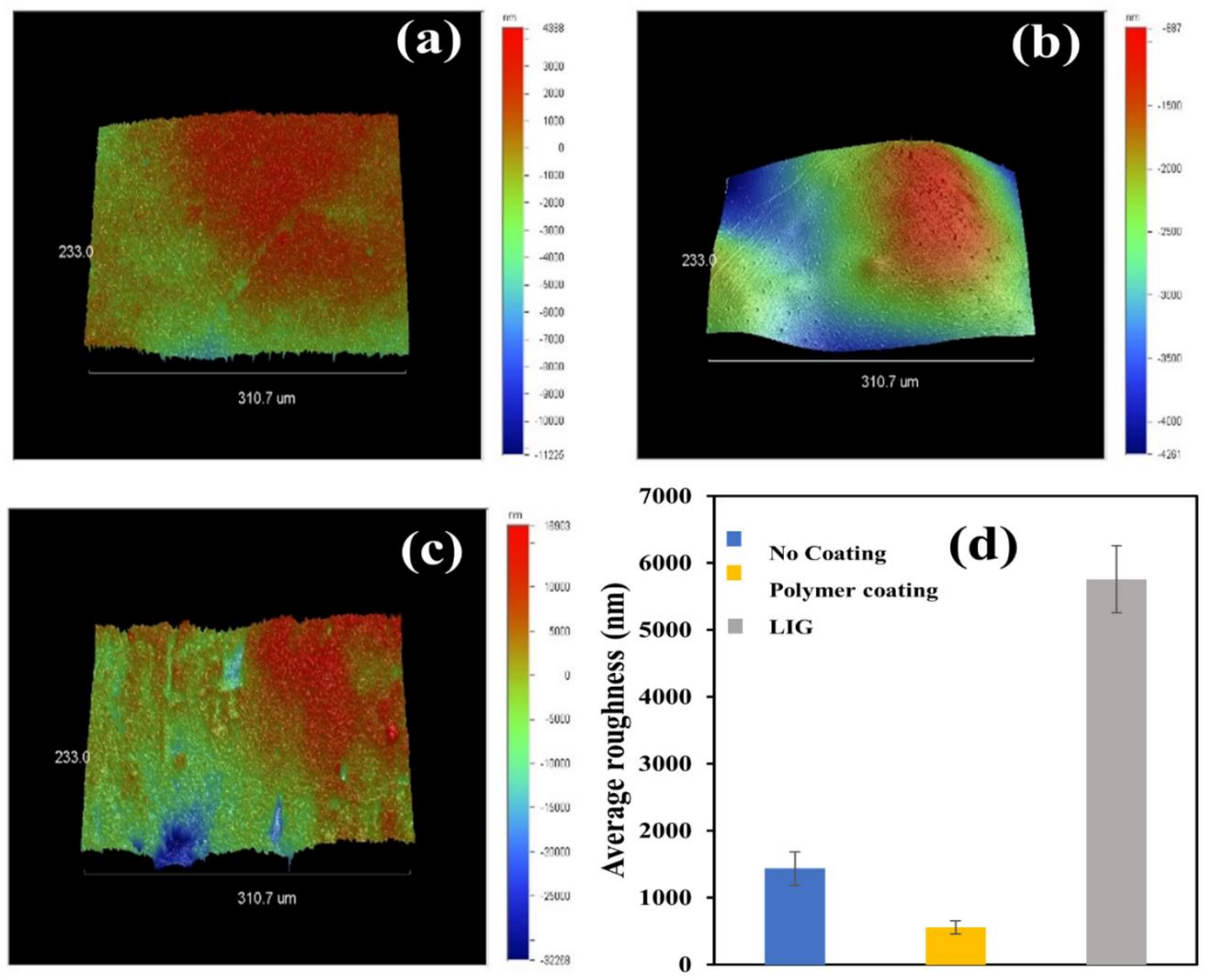

Figure 5.8 3D optical profilometry images of (a) a membrane surface with no PI or LIG coating. (b) a membrane surface with PI coating. (c) a membrane surface with LIG coating. (d) Data comparison of roughness values collected using optical profilometry of uncoated membrane surfaces, PI coated membrane surfaces, and LIG coated membrane surfaces. 
The laser parameters used for final membrane fabrication in this work $(10 \mathrm{~W}$ power, $0.61 \mathrm{~m} \mathrm{~s}^{-1}$ raster speed) were selected because they produced uniform and complete PI conversion to LIG, which was confirmed by SEM images. Other values tested for these parameters were not effective in producing a complete LIG layer on the surface of ceramic support.

The surface morphology of the support material before and after coating with PI was investigated by SEM and showed consistency with our previous preliminary work [199] (Figure 5.9). The effect of the number of PAA coating treatments on the thickness and integrity of the resulting LIG layer was investigated. The supports were coated with one, two, and four layers of PAA. After thermal imidization for the final number of coatings, the PI was transformed to LIG. When the supports were coated with one and two layers of PI, the ceramic grains were still partially seen in the surface (Figure 5.9(c, d)), and the process was not effective in producing a complete LIG layer. However, when four layers of PAA were applied, the PI was transformed to LIG (Figure 5.9(e)), that completely covered the surface and created a persistent layer. 

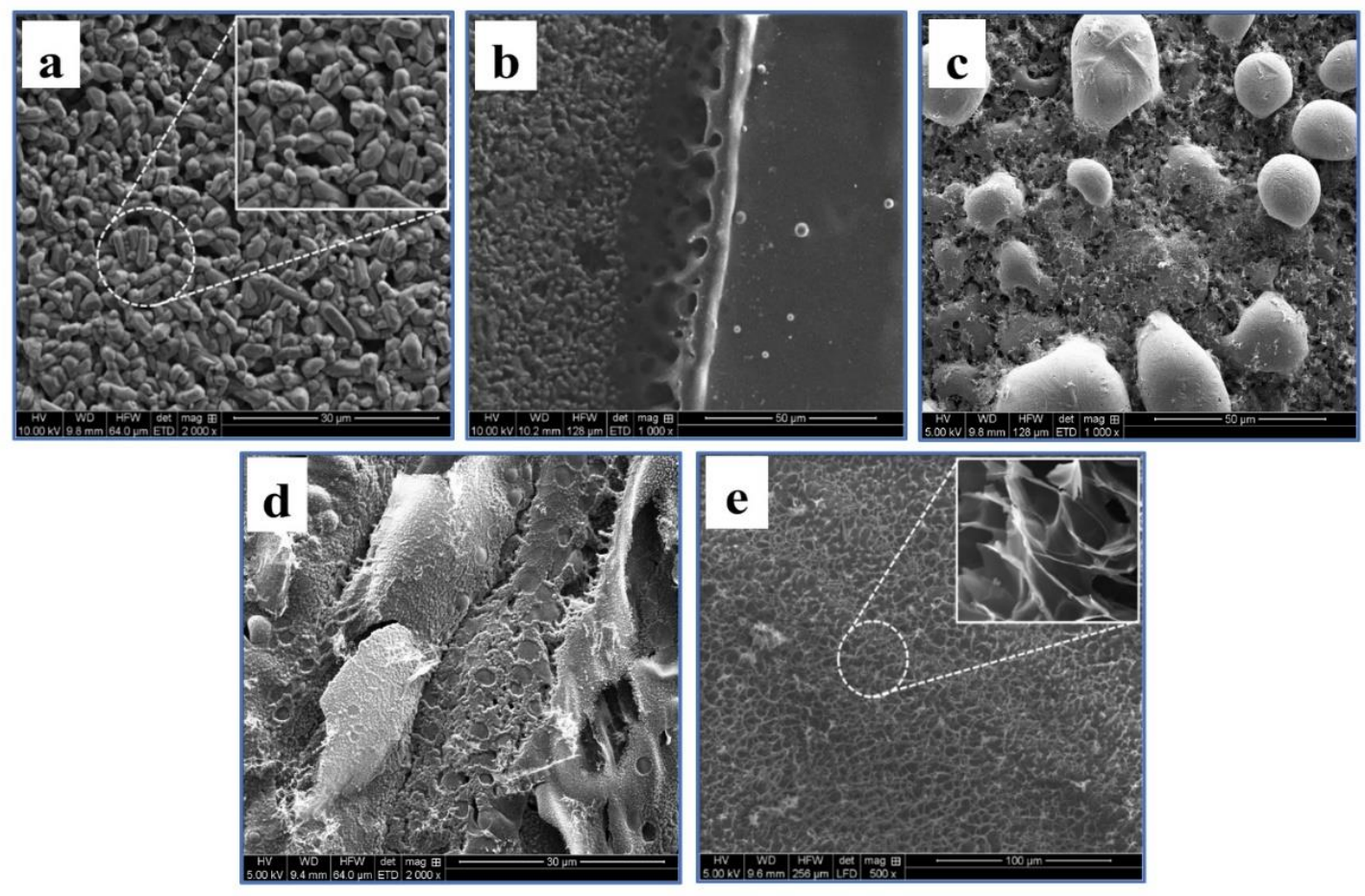

Figure 5.9 Scanning electron microscopy (SEM) of the ceramic support surface (a) before coating. (b) after coating with PI. (c) after one layer of PI was converted to LIG. (d) after two layers of PI were converted to LIG. (e) after four layers of PI were converted to LIG.

The open, sponge-like pore morphology of LIG observed is in agreement with the relatively high surface area measured by the nitrogen adsorption isotherms and the surface roughness determinations. Per the SEM images, the thickness of PI layer was $15 \pm 2 \mu \mathrm{m}$ (Figure $5.10(\mathrm{a}))$. The laser power selected $(10 \mathrm{~W})$ guaranteed conversion of PI to LIG with a thickness of $40 \pm 3 \mu \mathrm{m}$, while maintaining a PI layer of approximately $4 \mu \mathrm{m}$ to $6 \mu \mathrm{m}$ at the LIG-ceramic interface that acted as a root for LIG (Figure 5.10 (b)). This increase of coated layer thickness after conversion to LIG is due to the change of its morphology by the laser. Furthermore, the high temperature was capable of easily breaking the $\mathrm{C}-\mathrm{N}, \mathrm{C}-\mathrm{O}$, $\mathrm{C}=\mathrm{O}$ bonds found in $\mathrm{PI}$; as a result, $\mathrm{CO}_{2}$ gas is formed within the PI layer, producing the characteristic sponge-like structure of LIG as it is released [21]. High-resolution TEM 
image in Figure 5.11 revealed nano-shaped wrinkles at the exposed edges of graphene layers, due to the thermal expansion caused by laser irradiation [21]. The average lattice space of $3.4 \AA$ corresponds to the distance between two adjacent (002) planes in graphitic materials, similar to the value derived from XRD.
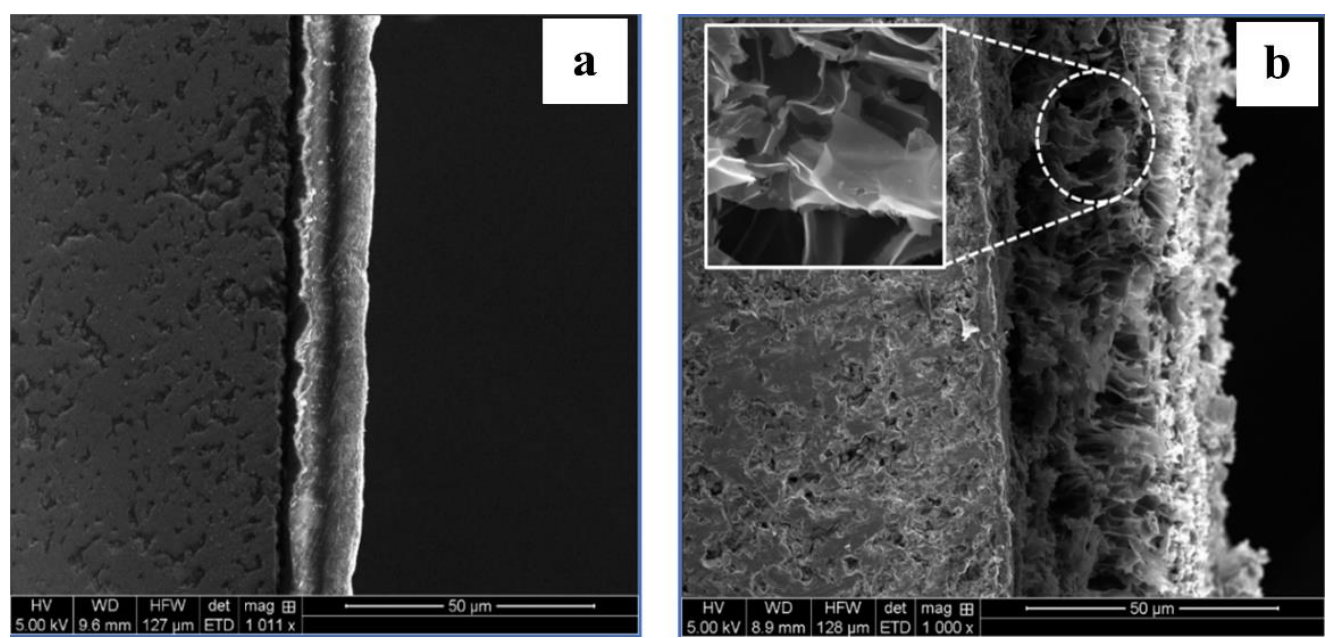

Figure 5.10 Scanning electron microscopy (SEM) of cross-section area (a) PI/ceramic membrane composite with PI thickness of $15 \pm 2 \mu \mathrm{m}$. (b) LIG/ceramic membrane composite with LIG thickness of $40 \pm 3 \mu \mathrm{m}$.

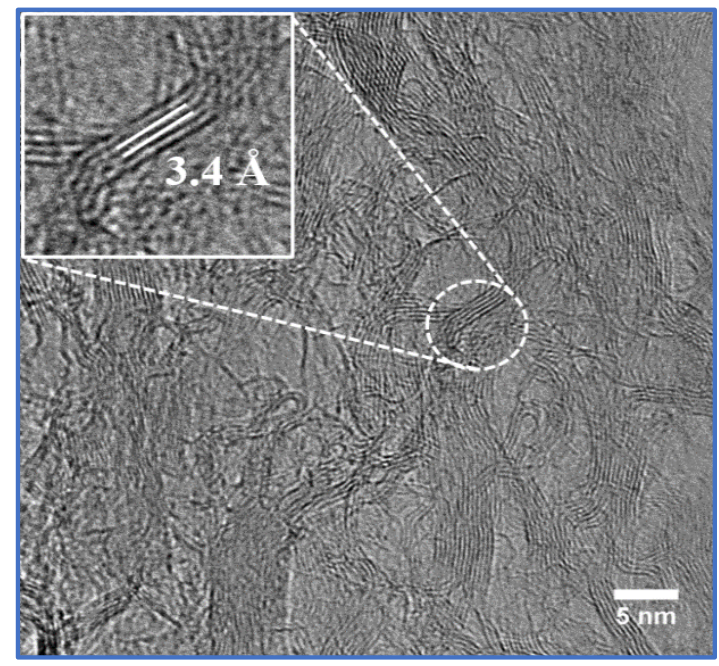

Figure 5.11 HRTEM image of LIG; scale bar, $5 \mathrm{~nm}$. Average lattice space of $\sim 3.4 \AA$, that corresponds to the (002) planes of graphitic materials. 


\subsubsection{Membrane Permeability}

Membrane hydrophobicity/hydrophilicity has been shown to affect its permeability, as this surface property may promote or repel different liquids [200]. Membrane-solvent interactions are expected to differ with changes in solvent properties such as viscosity, dipole moment, dielectric constant, surface tension, and molar volume. The original titanium oxide support material used in this work was highly hydrophilic; as a drop of water was placed on them during the contact angle (WCA) determination, it quickly entered the pore structure, but after LIG coating, a water contact angle (WCA) of $122^{\circ} \pm$ 5.4 was obtained (Figure 5.7). Test solvents were selected in order to capture a range of polarity and their pure fluxes through the membrane measured for LIG/CM at several trans-membrane pressures (TMP). Figure 5.12 shows the permeate flux for the selected solvents as a function of applied pressure, that consistently decreased at each TMP in the following order: hexane $>$ toluene $>$ isopropanol $>$ water, with permeability of $0.24,0.57$, 0.6, 2.4 $\mathrm{L} \mathrm{m}^{-2} \mathrm{~h}^{-1} \mathrm{kPa}^{-1}$ respectively. 


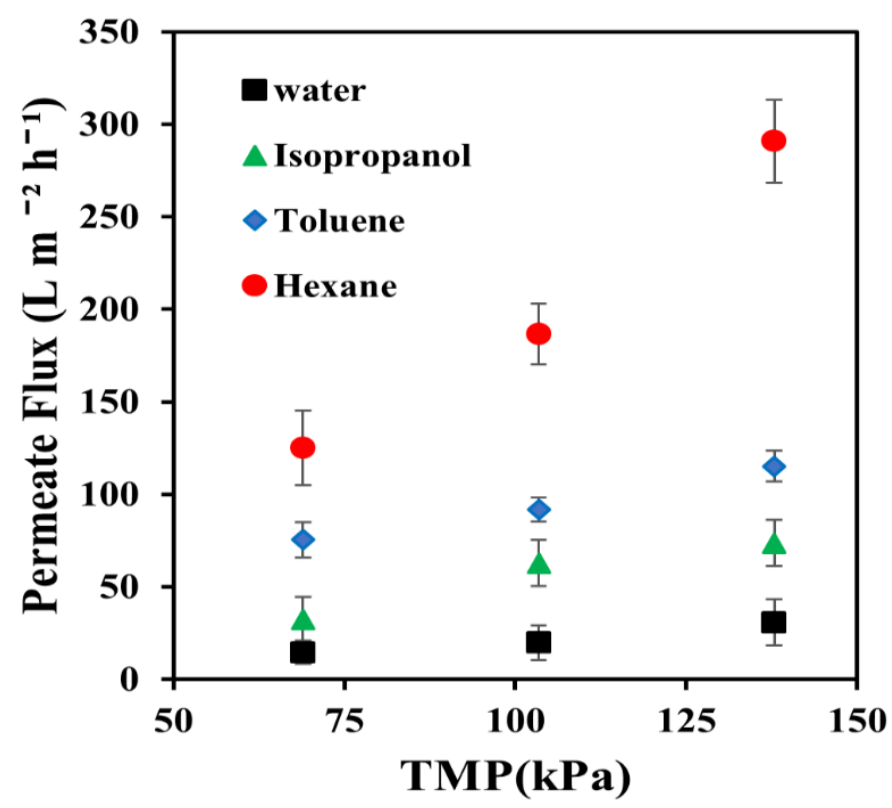

Figure 5.12 Pure solvent flux versus TMP for ceramic membrane coated with LIG at $20^{\circ} \mathrm{C}$.

Solvent transport could occur through the open pores or between graphene layers, depending on their molecular size. However, when considering the properties of the solvents (Table 5.1) and solvent fluxes (Figure 5.12), no clear correlation has been found between flux and solvent molecular volume. Hexane, with a molar volume (mv) of $130.58 \mathrm{~cm}^{3} \mathrm{~mol}^{-1}$, had higher flux than the smaller isopropanol $\left(\mathrm{mv}=76.9 \mathrm{~cm}^{3} \mathrm{~mol}^{-1}\right)$ and water $\left(\mathrm{mv}=18 \mathrm{~cm}^{3} \mathrm{~mol}^{-1}\right)$ [29, 30]. Moreover, Yang et al.[201] preformed XRD for highly laminated graphene oxide (HLGO) membranes immersed in different organic solvents and showed that nonpolar solvents with high permeability such as hexane, did not increase the interlayer distance $(d)$ of HLGO membranes, suggesting that the transport was not dominated by molecular transport through interlayer capillaries. Inverse behavior should be expected for LIG because of its hydrophobicity and transport of 
hydrophobic solvents through interlayer capillaries might be one of the permeation mechanisms for LIG membrane.

Higher fluxes were observed for nonpolar compounds (hexane and toluene) than for polar solvents (isopropanol and water). Clean water permeability was remarkably low $\left(0.24 \mathrm{~L} \mathrm{~m}^{-2} \mathrm{~h}^{-1} \mathrm{kPa}^{-1}\right.$ at $\left.20^{\circ} \mathrm{C}\right)$, while that of hexane was one order of magnitude higher $\left(2.4 \mathrm{~L} \mathrm{~m}^{-2} \mathrm{~h}^{-1} \mathrm{kPa}^{-1}\right.$ at $20^{\circ} \mathrm{C}$ ). This can be explained by the affinity of non-polar solvents for the hydrophobic surface of the membrane. All the solvents showed a consistent increase in flux with decreasing dipole moment and dielectric constant $(\varepsilon)$, representing solvent polarity, major parameters affecting solvent permeation through LIG membrane (Figure $5.13(a, b))$.

No correlation was found between surface tension $(\gamma)$ and flux for the solvents. However, low water fluxes were probably induced by the significant higher value of surface tension [186]. Except for the case of water, all other solvents showed a consistent increase in flux with decreasing viscosity values $(\mu)$. Machado et al. [186] showed that viscous forces are one of the major parameters affecting solvent flow through silicone uncharged polymeric membranes, and Liang et al.[202] reported that viscosity is the dominating factor in the permeability through reduced graphene oxide membranes. Dobrak et al. [187] have reported that viscosity and polarity are the most important parameters to examine the solvent flux through ceramic membranes. However, Yang et al. [203] showed that the difference in the flux through silicone uncharged polymeric membranes cannot be explained by differences in the surface tension and viscosity. Thus, the transport mechanism is not based on viscous flow through pores, and there must be interaction between the membrane and solvents. 

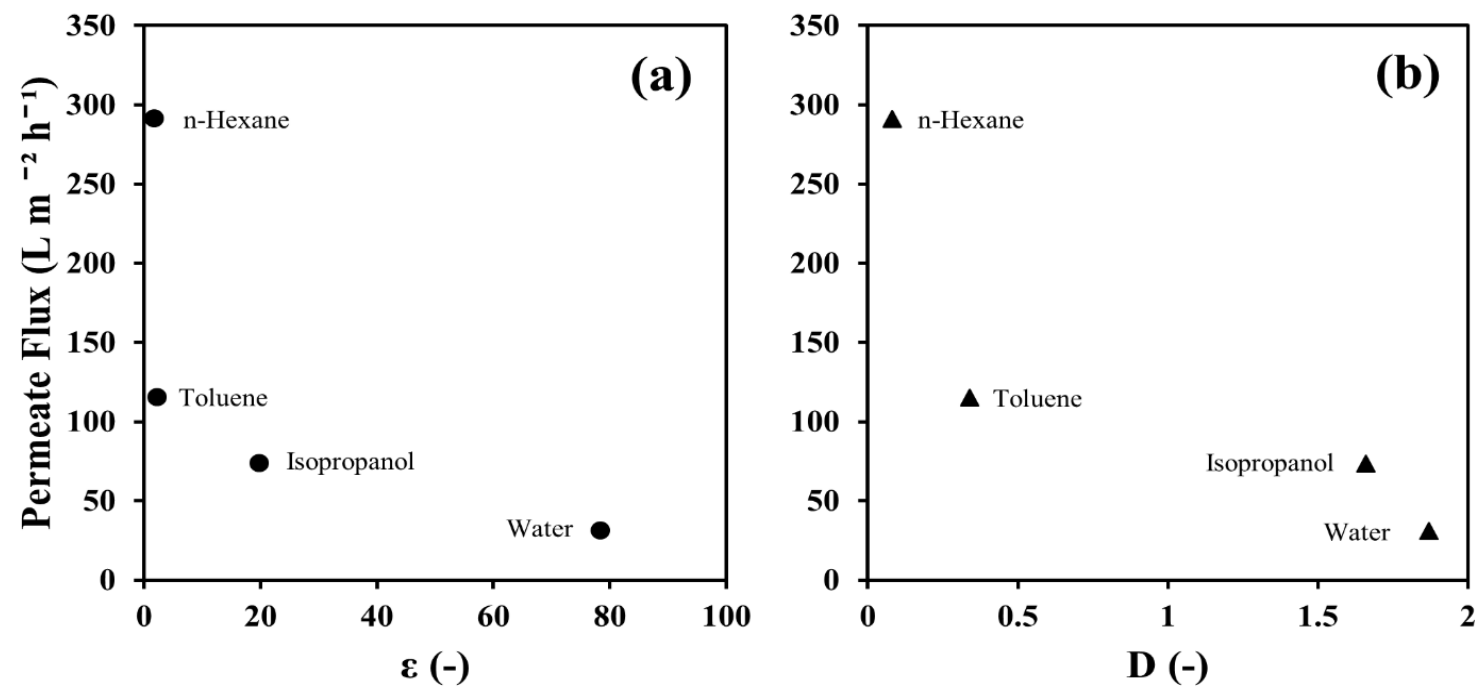

Figure 5.13 Permeate fluxes as a function of (a) dielectric constant. (b) dipole moment.

In summary, it can be concluded that the flux of the solvents depends on the membrane material and the properties of the solvent. If solvent transport in LIG/CM membrane were dominated by viscous-flow, all experiments should yield a similar permeability constant $k$, i.e. same slope $(k)$ in $J$ versus TMP/ $\mu$ plots [188], since it is assumed that membrane pore geometry is identical, regardless the type of solvent used. However, from the different slopes shown in Figure 5.14, it can be concluded that solvent viscosity is not the dominant factor in determining permeability in our experiments. Viscous-flow model does not take into consideration the interaction between the membrane surface and the permeating solvents and, for hydrophobic membranes, this interaction is much less important than for hydrophilic membranes, as a result of the absence of hydroxyl groups at the surface [187]. 


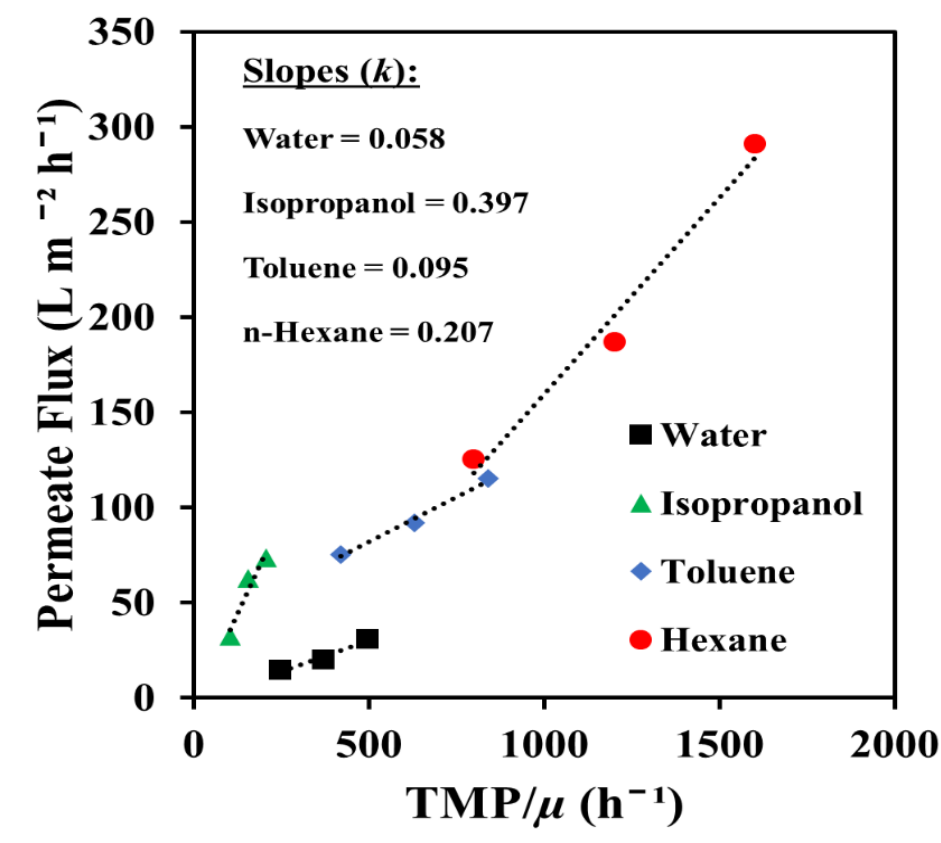

Figure 5.14 Fluxes versus TMP/ $\mu$ for $\mathrm{LIG}$ membrane at $20^{\circ} \mathrm{C}$.

Since the total Hansen solubility parameter $\left(\delta_{t o t}\right)$ consists of three components;

dispersion, polar, and H-bonding, representing the interaction strength and the polarity of the solvents, it has been used to explain polymeric (e.g. PDMS) and ceramic membranes performance $[189,204]$. One of the parameters not discussed clearly in the literatures is H-bonding capacity of the solvent-membrane system and its impact on the membrane performance. This parameter can be related to the H-bonding contribution of Hansen solubility parameter $\left(\delta_{h}\right)$ for the solvent[190] (Table 5.1). In a system with a hydrophilic membrane and a polar solvent, a high flux is expected due to high capability of $\mathrm{H}$ bonding between solvent and membrane (or vice versa) [205].

In order to investigate the H-bonding capability between solvents and LIG membrane, $J \times \mu$ was plotted as a function of H-bonding contribution of Hansen solubility parameter 
$\left(\delta_{h}\right)$ (Figure 5.15). The solvent with lowest H-bonding ability is supposed to have the highest flux; however, no clear trend between $J \times \mu$ and $\delta_{h}$ was observed indicating that the H-bonding is not the dominant factor. Other researchers have investigated the impact of the total Hansen solubility parameter $\left(\delta_{t o t}\right)$ on the hydrophobic ceramic membranes performance $[187,206]$. No trend was observed, suggesting a pure viscous flow through the membranes. However, our result showed that solvent viscosity is not the dominant factor in determining permeability, similarly to PDMS-grafted alumina membranes [188]. Thus, dipole moment and dielectric constant are the major factors controlling the transport through the LIG membrane.

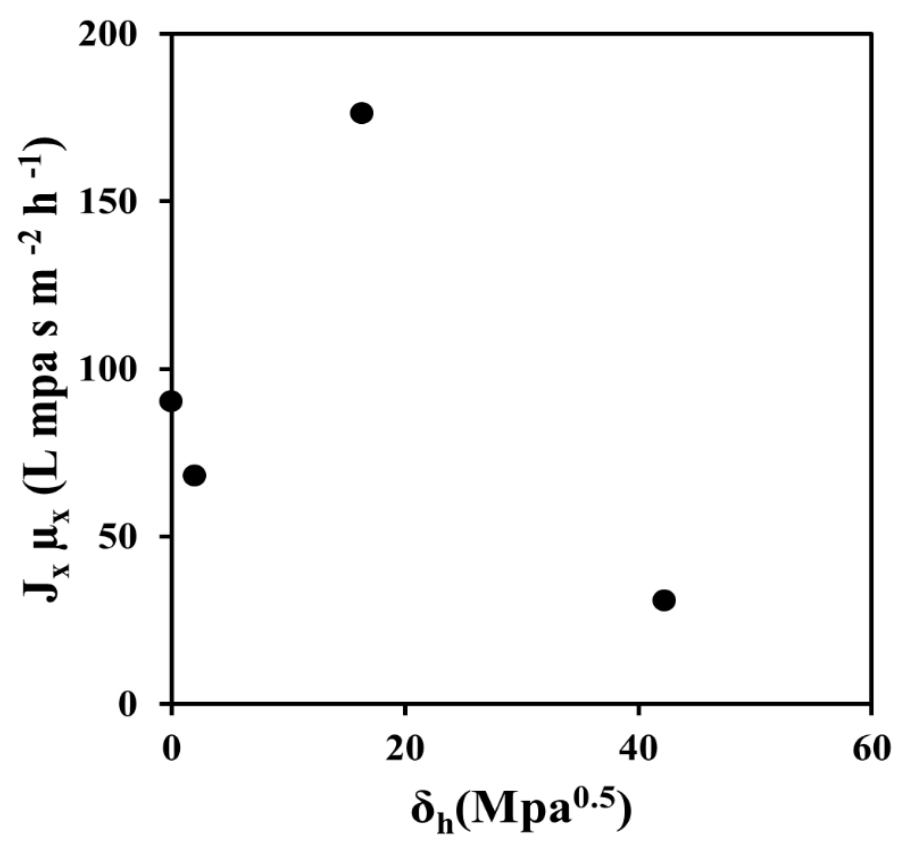

Figure 5.15 Flux and viscosity $\mathrm{J}_{\mathrm{x}} \times \mu_{\mathrm{x}}$ as a function of H-bonding contribution of Hansen solubility parameter $\delta_{h}$. Where x stands for solvent x. 


\subsubsection{Particle Rejection}

Selectivity is another essential factor for evaluating the performance of membranes [202]. In the characterization of a new membrane material, rejection experiments bring insight into the size distribution of effective/connective pores, as opposed to total porosity as given by $\mathrm{N}_{2}$ adsorption isotherm. Four different sizes of silica particles with size between $18.7 \pm 1.1 \mathrm{~nm}-255.6 \pm 9.5 \mathrm{~nm}$ were used to evaluate the separation performance of LIG/CM. Figure 5.16 presents rejection data for both uncoated ceramic membrane (UNCM) and LIG/CM. Filtration experiments conducted on the UNCM showed complete passage of silica particles in the feed solution, as expected based on their $1.4 \mu \mathrm{m}$ pore size. However, experiments of $\mathrm{LIG} / \mathrm{CM}$ showed partial rejection $5.6 \pm$ $2.2 \%, 25.7 \pm 4.6 \%, 38.8 \pm 1.8 \%$ for ST-O, ST-30-LH, ST-ZL respectively, but much higher rejection $(90.22 \pm 8.3 \%)$ for SP with average size of $255.6 \pm 9.5$. The nitrogen adsorption isotherms revealed a wide range of pore sizes with average of $54.55 \mathrm{~nm}$ (Figure 5.6 (b)). From the rejection results, it can be concluded that there were large connecting pores available for flow and particle transport, and that the rejection properties were dominated by that fraction of the porosity. 


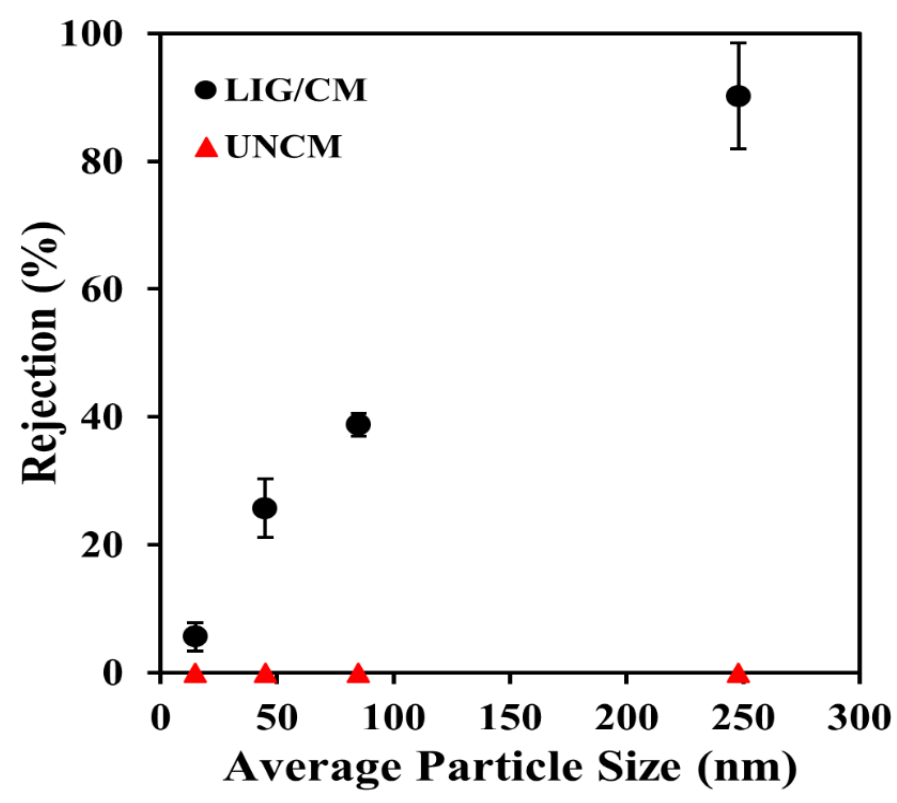

Figure 5.16 Particle rejection of uncoated ceramic membrane (UNCM) and LIG/CM vs. average particle size; test solutions were composed of silica particles suspended in methanol.

The availability of these channels supports the hypothesis of Hagen-Poiseuille cylindrical pore transport model through the LIG layer, as opposed to previously reported layered GO membranes, in which distance between layer edges and interlayer spacing become the characteristic pore size.

The development of the sponge-like structure of the LIG is a consequence to the laser treatment of the polymer (Figure 5.17 (a)) and leads to distinctive transport (permeability and rejection) properties from the compacted graphene and graphene oxide layers that deposited on support membrane by vacuum filtration (Figure 5.17 (b)) [202,207]. In fact, the root mean square (RMS) roughness value of GO membranes that prepared with vacuum filtration was reported to be $10.2 \mathrm{~nm}$ [207], much smaller than that of our LIG membrane $(5.8 \mu \mathrm{m})$, indicating the big differences in the surface morphology. Although 
the LIG layer shares some of the chemical properties of previously reported graphene membranes, its structure is radically different and gives the membrane unique characteristics for separation processes.

(a)

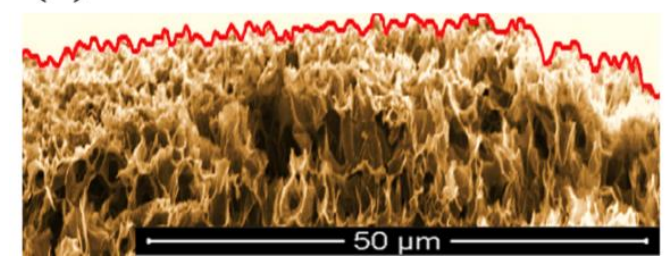

(b)

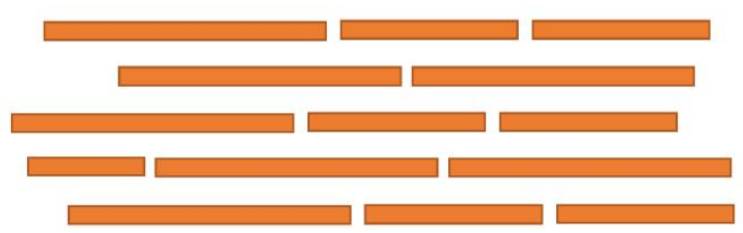

Figure 5.17 Schematic of the cross-section of membranes with effective layers composed of (a) Laser induced graphene (LIG); and (b) horizontally compacted graphene oxide (GO) sheets.

\subsection{Conclusion}

LIG/CM asymmetric membrane was successfully fabricated for the first time, forming strongly bonded graphene layer on top of the ceramic support. The characterization confirmed the imidization process and the formation of LIG on the surface of ceramic membrane. Permeate flux of solvents decreased in the following order: hexane $>$ toluene $>$ isopropanol $>$ water. Higher fluxes were observed for hexane and toluene (nonpolar solvents) than for isopropanol and water (polar solvents). All the liquids tested showed a consistent increase in flux with decreasing dipole moment and dielectric constant $(\varepsilon)$, representing solvent polarity, which might be the major parameters affecting solvent flow through LIG membrane. Filtration experiments showed that the LIG/CM had low rejection for particles ranged between $18.7 \pm 1.1 \mathrm{~nm}$ to $107 \pm 5.4 \mathrm{~nm}$; however, $90.22 \pm 8.3 \%$ rejection was achieved for particles with average size of $255.6 \pm$ $9.5 \mathrm{~nm}$. This result indicates that the fabricated LIG/CM had relatively large connecting 
pores, effective in separation processes, due to the sponge-like structure of graphene sheets.

The fabricated membrane has potential for the separation of liquid phases based on polarity and particles in the submicron range size, making it a suitable candidate for many applications such as oil/water separation or solvent recovery from wastewater. 


\section{Supporting Information}
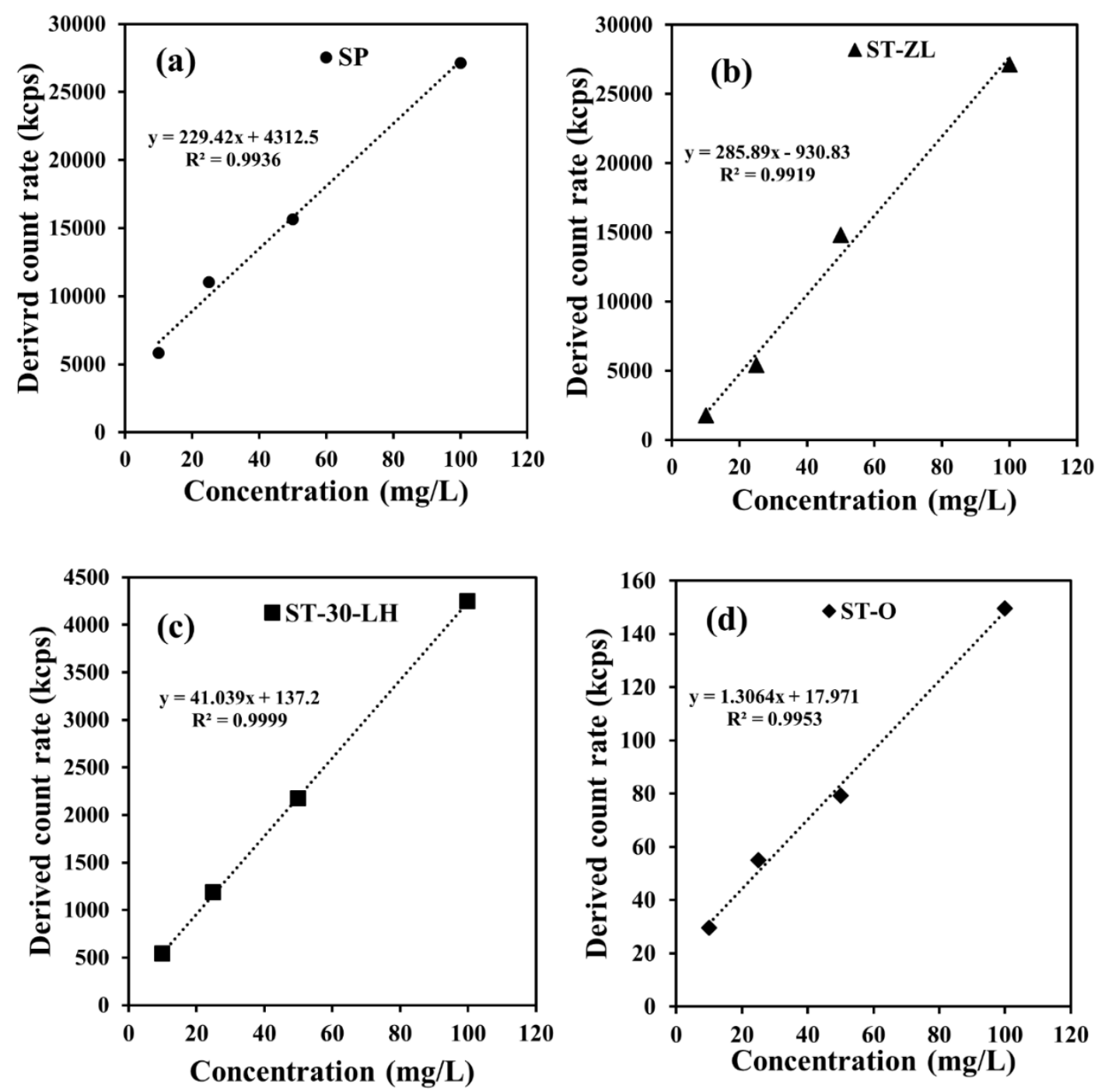

Figure 5.1s Calibration curves using DLS method for (a) SP with size of 230-265 nm. (b) ST-ZL with size of 70-100 nm. (c) ST-30-LH with size of 40-50 nm. (d) ST-O with size of 10-20 nm 


\title{
CHAPTER SIX
}

\section{Adsorption of Atrazine by Laser Induced Graphitic Material: An Efficient, Scalable and Green Alternative for Pollution Abatement}

\begin{abstract}
Laser induced graphitic material (LIG) was obtained from polyimide (PI) films using $\mathrm{CO}_{2}$ laser. This environmentally and economically promising fabrication method is a onestep, scalable, reagent-free process. LIG was fabricated, characterized, and tested as adsorbent for the removal of atrazine (ATZ) from water. The effect of presence of humic acid (HA), ionic strength (IS), adsorption time, initial ATZ concentration, and solution $\mathrm{pH}$ on the adsorption process was explored. The $\mathrm{N}_{2}$ Brunauer-Emmett-Teller (BET) surface area of LIG was $133.6 \pm 1.7 \mathrm{~m}^{2} / \mathrm{g}$. The prepared LIG exhibited significant removal of ATZ from aqueous solutions; hydrophobicity and $\pi$ - $\pi$ interactions played important roles in the process. Adsorption of ATZ on LIG followed a pseudo-second order kinetic model and Langmuir model for the isotherm with maximum adsorption capacity of $15.0 \mathrm{mg}$ ATZ/g LIG. Adsorption was more favorable at higher $\mathrm{pH}$ and not affected by ionic strength. LIG exhibited enhanced performance over many previously reported adsorbents. The introduction stage of HA with respect to ATZ influenced the results: pre-introduction of HA reduced ATZ adsorption by $32 \%$, whereas post-introduction of HA resulted in a slight release of ATZ from the LIG.

Keywords: Adsorption; Atrazine; Laser induced graphitic material; Humic acid
\end{abstract}




\subsection{Introduction}

Water contamination by agrochemicals is a substantial concern because of the health hazard towards humans and animals. Atrazine (2-chloro-4-ethylamino-6-isopropylaminos-triazine) has been extensively applied to control broad-leafed weeds and grasses in crop production [208]. In the United States alone, between 27 and 36 million kilograms of atrazine (ATZ) are used each year, mainly on corn crops in the Midwest. ATZ is often detected in ground and surface waters at concentrations above the legal limits because of its relative persistence and its high mobility in soils [209]. Its environmental half-life is up to 399 days in soils and 250 days in water [210], which raises concerns about human health and ecological impacts. Toxicological studies suggested that ATZ can interfere with endocrine hormone metabolism [211] and may cause liver cancer in humans [212]. In 2003, the European Union banned its application and established a strict concentration limit of $0.1 \mu \mathrm{g} / \mathrm{L}$ in drinking water while the corresponding maximum contaminant level in the US is $3 \mu \mathrm{g} / \mathrm{L}$ [213].

Surface interactions, in particular sorption and desorption processes, play a vital role in deciding the fate and transport of ATZ in the environment. Comprehensive studies can be found in the literature on ATZ sorption behavior on activated carbon, several nanomaterials, minerals, and soil [214-220]. Engineered nanostructured solids and/or nanoparticles usually provide higher adsorption capacities than natural colloids, but the safe and cost-effective synthesis of these materials have hindered their application in water treatment.

Nowadays, graphene-containing materials have acquired much attention due to their high surface area, active surface functional groups and physicochemical properties [221- 
223] and have shown to be efficient in removing a variety of pollutants from water [183]. For example, Yan et al. [224] found that chitosan/graphene oxide composite adsorbent (CS-GO) successfully removed various metal ions and dyes with different charge properties: anionic [methyl orange $(\mathrm{MO})$ and $\mathrm{Cr}(\mathrm{VI})]$ and cationic species [methylene blue (MB) and $\mathrm{Cu}(\mathrm{II})$ ]. L-Cysteine reduced graphene oxide (RGO-Cys) was found to be a great adsorbent of cationic neutral red (NR) and anionic indigo carmine (IC), with adsorption capacities of $1301.8 \mathrm{mg} / \mathrm{g}$ and $1005.7 \mathrm{mg} / \mathrm{g}$, respectively [225]. Furthermore, the adsorption of two organic compounds, biphenyl and phenanthrene, by granular activated carbon (GAC), single-walled carbon nanotubes (SWCNT), and multi-walled carbon nanotubes (MWCNT) was examined and compared with graphene oxide (GO) and two pristine graphene nanosheets (GNS) in the presence of natural organic matter (NOM). Graphene exhibited better adsorption capacities than GAC and carbon nanotubes (CNT). Additionaly, the NOM effect on adsorption was less pronounced on graphene than on the other carbon based materials [226].

Graphene oxide is most often synthesized following the original Hummers' method [18] or slightly modified protocols [19,20]. However, these methods use hazardous chemicals and strong oxidants. GO particles need to be assembled in some convenient way for the treatment process. The assembly of GO into foams was used as a conventional method for the fabrication of 3D graphene-based materials, but again this method needs the GO precursor to be prepared through its oxidative hazard synthesis route $[99,100]$. Another route to produce 3D graphene is chemical vapor deposition (CVD) on porous substrates, but the drying process and subsequent etching and high temperature conditions may inhibit its scalable production $[102,103,105,227,228]$. In contrast to the 
aforementioned processes, laser induced graphitic material (LIG) is obtained by laser irradiation of a polyimide (PI) film under ambient conditions, in a one-step, scalable, reagent-free process, which makes it an environmentally and economically sound alternative [21]. Several studies showed that different types of CNTs are toxic to zebrafish embryos and mammalian cells [229-231]. However, a recent study by d'Amora et al. [232] that investigated the toxicity of LIG through zebrafish model, showed that high concentration of LIG does not exhibit toxic effects and does not interfere with zebrafish development.

In the present study, LIG was fabricated and investigated for the first time as adsorbent for water treatment. The material was extensively characterized, and batch experiments were conducted to investigate the adsorption of ATZ. The impact of humic acid (HA) and different adsorption conditions were studied: solution $\mathrm{pH}$, ionic strength (IS), adsorption time, and initial adsorbate concentration.

\subsection{Material and methods}

\subsubsection{Chemicals}

Atrazine (ATZ), ethanol (99.5\%) and sodium nitrate $\left(\mathrm{NaNO}_{3}\right)$ were purchased from Sigma-Aldrich, St. Louis, MO, USA. Methanol (MeOH), acetonitrile (ACN) (HPLC grade), hydrochloric acid $(\mathrm{HCl})$ and sodium hydroxide $(\mathrm{NaOH})$, used for $\mathrm{pH}$ adjustments, were purchased from Fisher Scientific, Pittsburgh, PA, USA. Finally, a technical grade humic acid (HA) was purchased from Acros, Geel, Belgium. 


\subsubsection{Preparation of laser induced graphitic material (LIG)}

Figure 6.1 shows the general preparation procedure of LIG. The details of the preparation have been reported in previous publications [199,233]. In brief, the starting material was a commercially available PI film (Kapton ${ }^{\circledR}$, thickness $127 \mu$ m, McMasterCarr, USA). The $\mathrm{sp}^{3}$-carbon atoms were photothermally converted to $\mathrm{sp}^{2}$-carbon by irradiating the PI using a computer-controlled $\mathrm{H}$-series Desktop $\mathrm{CO}_{2}$ laser (Full Spectrum, Las Vegas, NV) (beam size: $\approx 100 \mu \mathrm{m}$, pulse duration: $\approx 14 \mu$ s, wavelength: $10.6 \mu \mathrm{m}$, maximum scanning speed: $\approx 60 \mathrm{~cm} \mathrm{~s}^{-1}$, maximum power: $40 \mathrm{~W}$ ). The setting was fixed at $25 \%$ of the maximum power and $25 \%$ of the maximum scanning speed. The dots per inch (DPI) was set to 1,000. The PI film morphology and chemical structure is fundamentally changed by the laser incidence. The high temperatures produced broke the $\mathrm{C}-\mathrm{N}, \mathrm{C}-\mathrm{O}, \mathrm{C}=\mathrm{O}$ bonds in $\mathrm{PI}$ and gases were released, which caused the sponge-like structure of LIG and wrinkles due to thermal expansion. Free standing LIG particles were obtained scraping the surface of the PI using a flat end spatula.

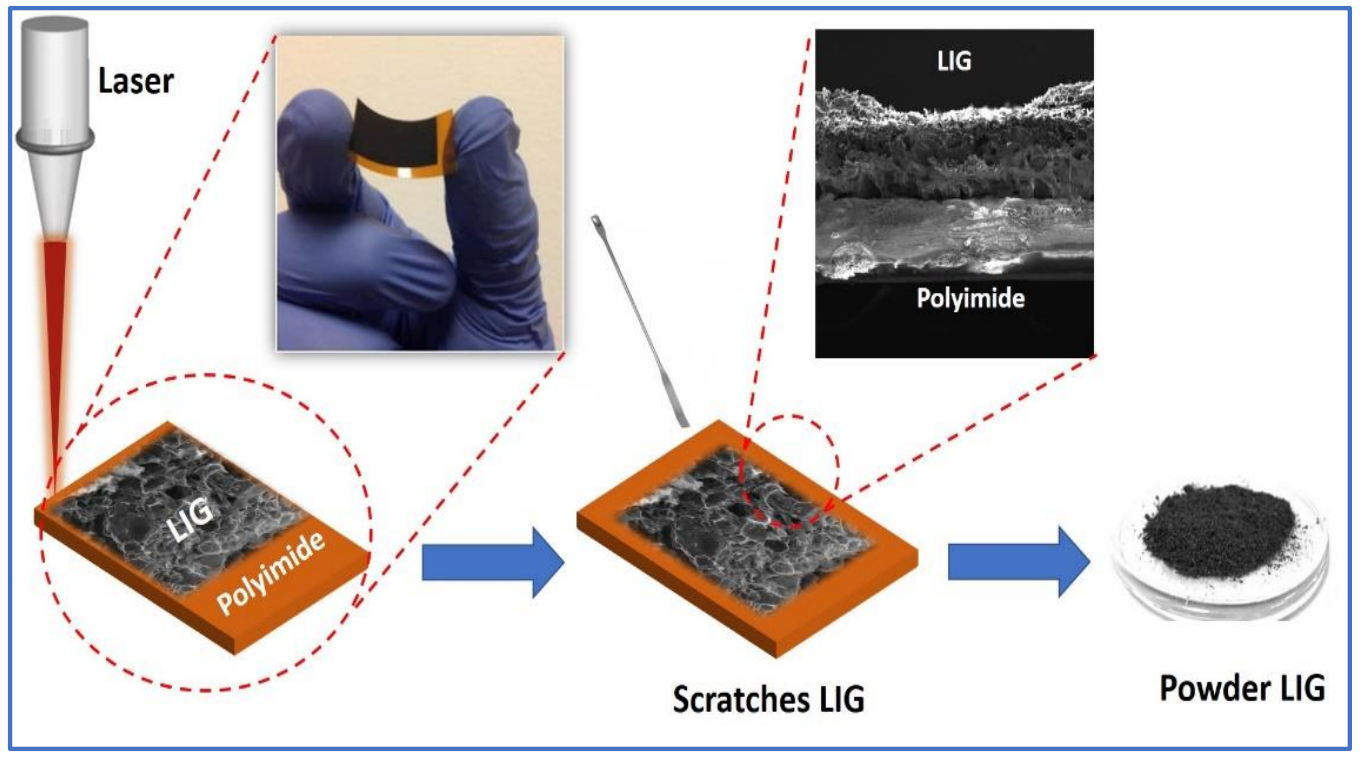

Figure 6.1 General fabrication procedure of laser induced graphitic material (LIG). 


\subsubsection{Characterization of LIG powder}

The thickness and the morphology of LIG and PI films were observed with a FEI Quanta 600 FEG environmental scanning electron microscopy (ESEM) (ThermoFisher Scientific, Hillsboro, OR, USA). In order to capture high resolution images of the LIG flakes, a Tecnai F30 twin transmission electron microscopy (TEM) (ThermoFisher Scientific, Hillsboro, OR, USA) was used. Brunauer-Emmett-Teller (BET) specific surface area was measured by a nitrogen adsorption surface area analyzer (SA 3100, Beckman Coulter, USA). The surface chemistry of PI and LIG was analyzed by FourierTransform Infrared Spectroscopy (FTIR), in a Cary 660 FTIR spectrophotometer (Agilent Technologies, Santa Clara, CA, USA). The spectra were obtained from $\mathrm{KBr}$

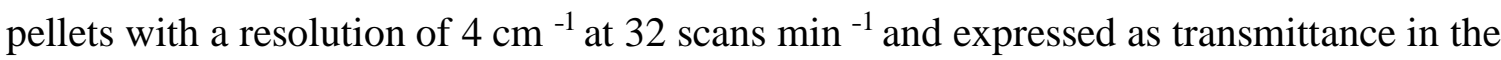
400-4000 min $^{-1}$ range. A PANanlytical XPert Pro, the Netherlands, with $\mathrm{Cu} \mathrm{K} \alpha$ radiation $(\lambda=1.54 \AA)$ was used to conduct the X-Ray Diffraction (XRD) analysis of LIG. The samples were scanned from $10^{\circ}$ to $50^{\circ}(2 \theta)$ with a step size of 0.025 and account time of 1s at each point. X-ray photoelectron spectroscopy (XPS) was performed in ultrahigh vacuum (Kratos Axis 165, Manchester, UK) using a monochromatic Al Ka source (hv = $1486.6 \mathrm{eV}$, resolution $=0.5 \mathrm{eV}$ ). Raman spectra was collected with a Renishaw inVia (Gloucestershire, UK) Raman microscope using 633-nm laser excitation at room temperature with a laser power of $5 \mathrm{~mW}$. The zeta potential $(\zeta)$ of LIG was measured using a ZetaSizer Nano ZS (Malvern Instruments, Worcestershire, U.K.), from electrophoretic mobility (EPM) determinations converted to $\zeta$ by the Smoluchowski equation (Equation 6.1) [234]: 


$$
E P M=\frac{\varepsilon \zeta}{\mu}
$$

where $\varepsilon$ is the dielectric constant of the solution and $\mu$ is its viscosity. Disposable folded capillary cells were employed.

\subsubsection{Analytical Method}

The concentration of ATZ was analyzed by High-Performance Liquid Chromatography (HPLC) in a Shimadzu LC-2010A HT (Kyoto, Japan), equipped with a Kinetex® C18 stainless steel column (Phenomenex, Torrance, CA, USA) with $100 \mathrm{~mm}$ length $\times 4.6 \mathrm{~mm}$ internal diameter, and $2.6 \mu \mathrm{m}$ particles size. ATZ was eluted from the column at flow rate of $0.5 \mathrm{~mL} \mathrm{~min}^{-1}$ at $40{ }^{\circ} \mathrm{C}$. The system was first washed with $100 \%$ methanol, and the column was washed with $95 \%$ acetonitrile and $5 \%$ phosphoric acid (1\%) solution. The detection was performed by UV-Vis absorption at a wavelength of $223 \mathrm{~nm}$; the injection volume was $20 \mu \mathrm{L}$ [235].

\subsubsection{Adsorption experiments}

Adsorption experiments were carried out to investigate the effect of time, initial adsorbate concentration, solution $\mathrm{pH}$, IS and presence of HA. Stock solution of $20 \mathrm{mg} / \mathrm{L}$ ATZ and variable ionic strength were prepared in ultrapure water $\left(18.2 \mathrm{~m} \Omega . \mathrm{cm}\right.$ at $25^{\circ} \mathrm{C}$, Thermo Scientific ${ }^{\mathrm{TM}}$ Barnstead $^{\mathrm{TM}}$ E-Pure ${ }^{\mathrm{TM}}$ Ultrapure Water Purification System, Waltham, MA, USA) containing $1 \mathrm{mM}, 10 \mathrm{mM}$, and $100 \mathrm{mM}$ of $\mathrm{NaNO}_{3}$, respectively. Then, $10 \mathrm{mg}$ of LIG was added to $50 \mathrm{ml}$ of a series of ATZ solutions with concentrations $10,8,5,2.5,1 \mathrm{mg} / \mathrm{L}$. The $\mathrm{pH}$ was adjusted to 3,6 and 9 by addition of 0.1 
$\mathrm{M} \mathrm{NaOH}$ or $0.1 \mathrm{M} \mathrm{HCl}$. The flasks were sealed and shaken at $200 \mathrm{rpm}$ at ambient temperature for $48 \mathrm{~h}$. Then, aliquots were taken and filtered immediately through $0.2 \mu \mathrm{m}$ syringe filter (Whatman ${ }^{\circledR}$ Anotop $^{\circledR}$ ).

To investigate the impact of HA on ATZ adsorption by LIG, batch adsorption experiments were conducted in the presence of $5 \mathrm{mg} / \mathrm{L} \mathrm{HA}$ at $\mathrm{pH}=6$ and $10 \mathrm{mM} \mathrm{NaNO}$. Furthermore, the competitive adsorption of ATZ and HA was investigated; ATZ was mixed with HA for $48 \mathrm{~h}$, then LIG was added and further agitated to reach equilibrium time. ATZ was analyzed in samples that were either pre- or post-exposed to HA. For preHA exposure, LIG was first mixed in a HA solution for $48 \mathrm{~h}$, then the solution was spiked with ATZ and was further mixed for the required equilibrium time. Likewise, post-HA exposure experiments were accomplished by mixing the LIG in presence of ATZ, then HA was added by spiking. To assess the desorption (regeneration) process, ATZ loaded LIG was suspended in $50 \mathrm{ml}$ ethanol and the resulting mixture was agitated for $24 \mathrm{~h}$ at room temperature. The mixture was centrifuged in an Eppendorf $5810 \mathrm{R}$ centrifuge (Hauppauge, NY, USA) at 10,000 rpm for $30 \mathrm{~min}$.

The ATZ concentrations were determined by HPLC; the percentage of ATZ adsorbed by LIG was calculated using Equation (6.2):

$$
\% \text { Adsorption }=\frac{\left(C_{0}-C_{t}\right)}{C_{0}} \times 100
$$

where $C_{0}$ is the initial concentration $(\mathrm{mg} / \mathrm{L})$ and $C_{t}$ is the concentration after a time $t$ $(\mathrm{mg} / \mathrm{L})$.

The adsorption kinetic experiments were performed in $125 \mathrm{ml}$ conical flasks where 40 mg of LIG was added to $100 \mathrm{ml}$ of a $5 \mathrm{mg} / \mathrm{L}$ solution of atrazine at $\mathrm{pH}$ 6. Samples were 
taken at predetermined time intervals from $5 \min$ to $72 \mathrm{~h}$. The amount adsorbed at any time was determined by Equation (6.3):

$$
q_{t}=\left(C_{0}-C_{t}\right) \times \frac{V}{m}
$$

where $q_{t}, V$ and $m$ are the amount of ATZ adsorbed by LIG (mg ATZ/g LIG) at time $t$, volume of the solution (L), mass ( $\mathrm{g}$ ) of LIG, respectively.

The relevant physicochemical properties of ATZ are shown in Table 6.1.

Table 6.1 Physiochemical properties of ATZ [218].

\begin{tabular}{lcc}
\hline \multicolumn{1}{c}{ Parameter } & $\mathrm{C}_{8} \mathrm{H}_{14} \mathrm{ClN}_{5}$ \\
\hline Molecular formula & 215.68 \\
Molecular weight $(\mathrm{g} / \mathrm{mole})$ & $28 @ 25^{\circ} \mathrm{C}$ \\
Water solubility $(\mathbf{m g} / \mathbf{L})$ & 12.3 \\
pk & 2.61 \\
Log Kow & 0.96 \\
Molecular size: & 0.84 \\
Length (nm) & 0.3 \\
Width (nm) &
\end{tabular}

\subsubsection{Data analysis}

Pseudo-first-order Equation (6.4) and pseudo-second-order kinetic Equation (6.5) models were used to fit the experimental data:

$$
\begin{gathered}
\ln \left(q_{e}-q_{t}\right)=\ln \left(q_{e}\right)-k_{1} t \\
\frac{t}{q_{t}}=\frac{1}{q_{e}^{2} k_{2}}+\frac{t}{q_{e}}
\end{gathered}
$$


where $k_{l}\left(\mathrm{~h}^{-1}\right)$ is the rate coefficient of pseudo-first-order adsorption, $k_{2} \mathrm{~g} /(\mathrm{mg} \mathrm{h})$ is the rate coefficient of pseudo-second-order, and $q_{e}$ and $q_{t}$ are amount adsorbed $(\mathrm{mg} / \mathrm{g})$ at equilibrium and at time t, respectively.

The intraparticle diffusion model (IPD) suggested by Weber and Morris [236], which assumes that adsorption kinetics is controlled by diffusion mechanism, was used to determine the rate-limiting step of the adsorption process (Equation 6.6).

$$
q_{t}=k_{i}\left(t^{1 / 2}\right)+C
$$

where $k_{i}$ is the intraparticle diffusion rate constant $\left(\mathrm{mg} /\left(\mathrm{g} \mathrm{h}^{1 / 2}\right)\right.$, and $C(\mathrm{mg} / \mathrm{g})$. A linear relationship between $q_{t}$ and $t^{1 / 2}$ indicates that intraparticle diffusion is one of the mechanisms controlling the kinetics of adsorption, while a null value of $\mathrm{C}$ indicates that it dominates over diffusion through the liquid boundary layer. A multi-step adsorption process would produce several linear regions in a plot of $q_{t}$ versus $t^{1 / 2}$ [237]. Freundlich and Langmuir isotherm models, commonly applied to describe adsorption isotherms, were used to fit equilibrium adsorption data. The Langmuir isotherm assumes monolayer adsorption on a homogenous surface with a finite number of adsorption sites and no further adsorption can take place once a site is occupied. The Langmuir model can be expressed as:

$$
q_{e}=\frac{q_{\max } K_{L} C_{e}}{1+K_{L} C_{e}}
$$

where $q_{e}(\mathrm{mg} / \mathrm{g})$ is the amount of ATZ adsorbed by mass of LIG, $C_{e}(\mathrm{mg} / \mathrm{L})$ is the equilibrium concentration of atrazine in the solution, $q_{\max }$ is the maximum adsorption capacity $(\mathrm{mg} / \mathrm{g})$ and $K_{L}$ is the Langmuir constant $(\mathrm{L} / \mathrm{mg})$ related to the bonding force of sorption. The Freundlich isotherm is appropriate for multilayer adsorption and is based 
on the assumption of a heterogeneous adsorbent surface [238]. The Freundlich isotherm is mathematically described as:

$$
q_{e}=K_{F} C_{e}^{1 / n}
$$

where $K_{F}$ is the adsorption equilibrium constant $\left[(\mathrm{mg} / \mathrm{g})(\mathrm{mg} / \mathrm{L})^{-\mathrm{n}}\right]$ representing the adsorption capacity, and $n$ is a constant indicative of adsorption intensity.

\subsection{Results and discussions}

\subsubsection{LIG powder characterization}

The $\mathrm{N}_{2}$ adsorption - desorption isotherms obtained corresponded to a type IV, with type H3 hysteresis loop [198], thereby suggesting the presence of mesopores (Figure 6.2). Barrett-Joyner-Halenda (BJH) method was used to obtain the pore size distribution curve. The average pore diameter of LIG was found to be $38 \pm 28 \mathrm{~nm}$ and its specific surface area $133.6 \pm 1.71 \mathrm{~m}^{2} / \mathrm{g}$

The point of zero charge ( $\mathrm{pH} \mathrm{PZC})$ is an important parameter to determine the adsorbent surface charge and its sensitivity to $\mathrm{pH}[239$ ]. Figure 6.3 shows the $\zeta$ of LIG as a function of $\mathrm{pH}$. From these measurements, the $\mathrm{pH}_{\mathrm{PZC}}$ was determined to be $\mathrm{pH}=3.5$, indicating the presence of functional groups with significant acidity. 


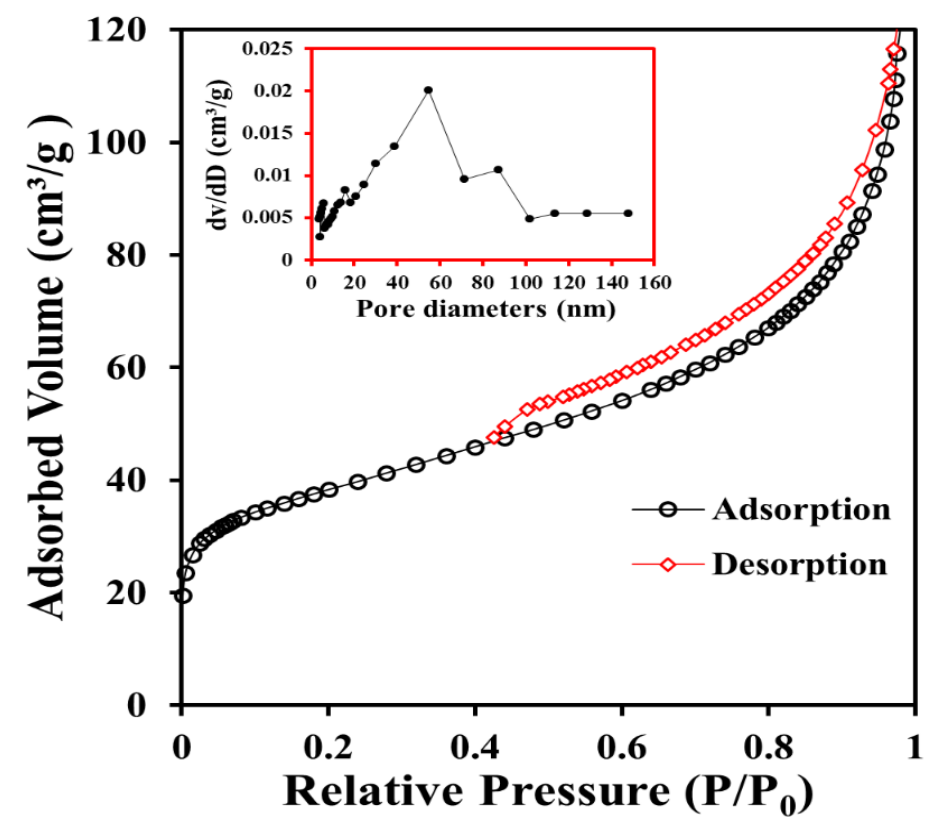

Figure 6.2 $\mathrm{N}_{2}$ adsorption isotherm and pore size distribution (insert) of laser induced graphitic material (LIG).

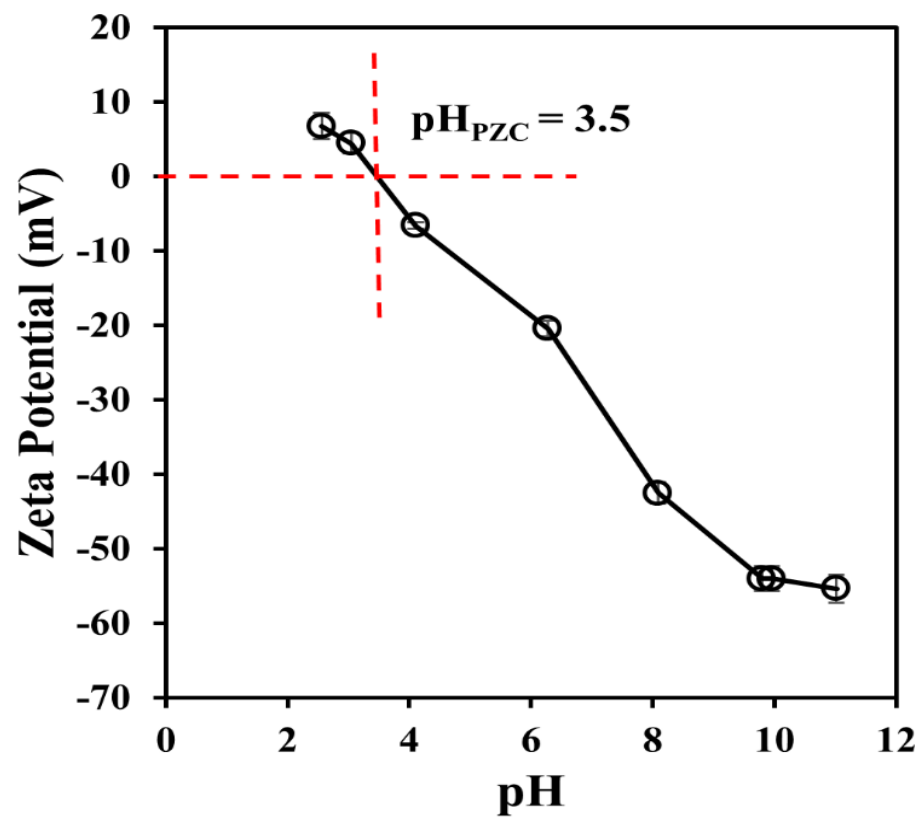

Figure 6.3 Zeta potential of laser induced graphitic material (LIG) as a function of $\mathrm{pH}$. 
The presence of graphene in the obtained sample is evidenced by the Raman spectrum (Figure 6.4), including a D peak at $\sim 1,350 \mathrm{~cm}^{-1}$, a $\mathrm{G}$ peak at $\sim 1,582 \mathrm{~cm}^{-1}$ and a 2D peak at $\sim 2,700 \mathrm{~cm}^{-1}$. The $\mathrm{D}$ peak is suggestive of defects or bent $\mathrm{sp} 2$-carbon bonds, whereas $\mathrm{G}$ peak is the first-order Raman band of all $\mathrm{sp}^{2}$ hybridized carbon. The 2D peak originates from second order zone boundary phonons [21,197].

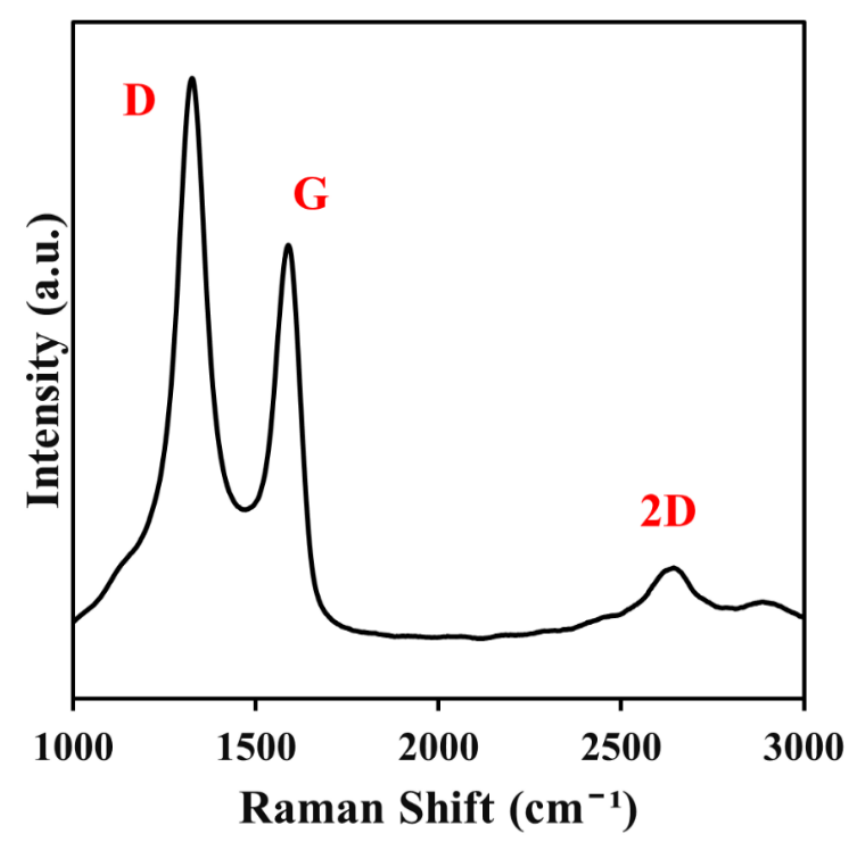

Figure 6.4 Raman spectrum of laser induced graphitic material (LIG) film obtained from $10 \mathrm{~W}$ laser power and $15 \mathrm{~cm} / \mathrm{s}$ raster speed.

The XRD analysis showed a strong peak at $2 \theta=25.9^{\circ}$, from which an interlayer spacing of $3.4 \AA$ between (002) planes in the LIG can be estimated, confirming the crystalline structure of graphite. Another peak at $2 \theta=42.9^{\circ}$ indicated (100) reflections which are associated with an in-plane structure [21] (Figure 6.5). 


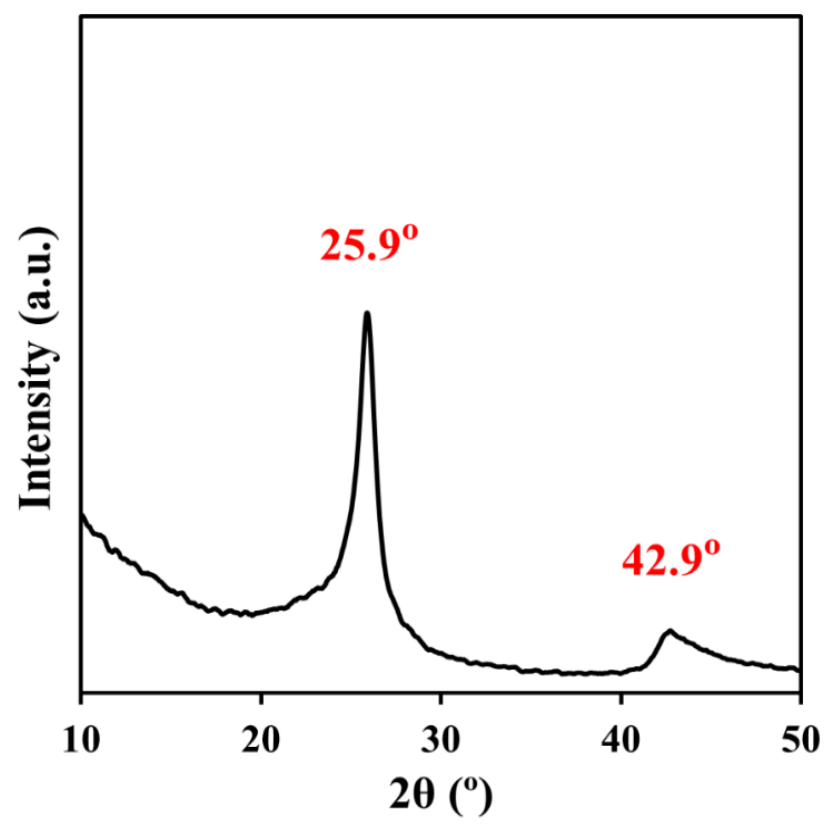

Figure 6.5 XRD patterns of powdered laser induced graphitic material (LIG).

FTIR spectrum (Figure 6.6) of PI reveals absorption bands at $1777 \mathrm{~cm} \cdot(\mathrm{C}=\mathrm{O}$ asymmetric stretching of imide), $1725 \mathrm{~cm}(\mathrm{C}=\mathrm{O}$ symmetric stretching $), 1374 \mathrm{~cm}(\mathrm{C}-\mathrm{N}$ stretching $)$ and $723 \mathrm{~cm}^{-}(\mathrm{C}=\mathrm{O}$ bending of imide $)[195,196]$. After the laser scribing, a broad absorption from $400 \mathrm{~cm}^{-1}$ to $2000 \mathrm{~cm}^{-1}$ shows a large variation in the spectrums. LIG spectrum exhibited two small peaks, at $1510 \mathrm{~cm}^{1}$ which is the tension band $\mathrm{C}=\mathrm{C}$ characteristic of the double bonds in the polycyclic aromatic graphene ring and at 1220 $\mathrm{cm}^{-1}$ corresponding to $\mathrm{C}-\mathrm{O}$ stretch. 


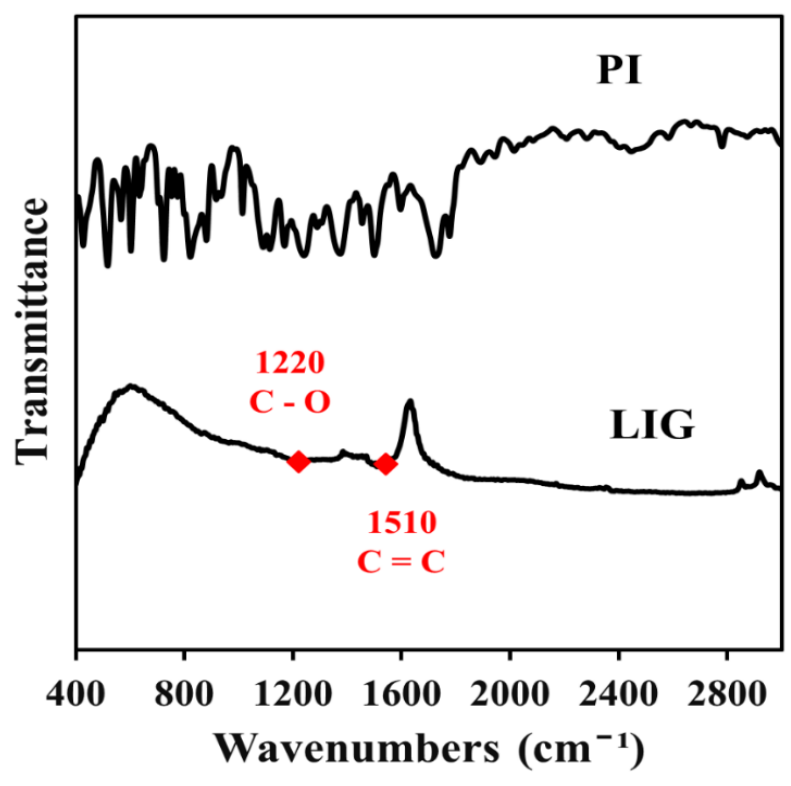

Figure 6.6 FTIR spectroscopy of polyimide (PI) and laser induced graphitic material (LIG).

The morphology of LIG as it was formed on the PI film surface was investigated using SEM (Figure 6.7 (a)). Figure 6.7 (b) shows the cross-sectional view of PI and LIG. The laser settings $(15 \mathrm{~cm} / \mathrm{s}$ raster speed, $10 \mathrm{~W}$ power) were selected so as to ensure conversion of PI to LIG with thickness of $107.4 \pm 3.8 \mu \mathrm{m}$ [199].
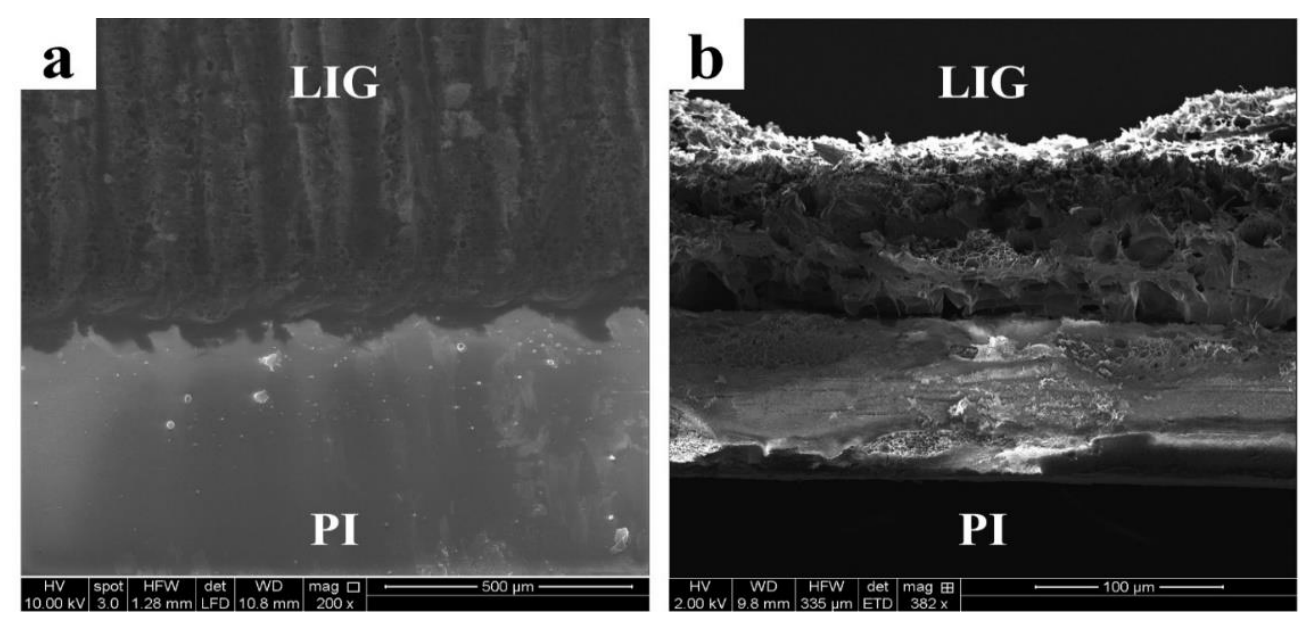

Figure 6.7 Scanning electron microscopy (SEM) of (a) the surface of PI and LIG. (b) crosssection view of PI/LIG composite with LIG thickness of $107.4 \pm 3.8 \mu \mathrm{m}$. 
Figure 6.8 (a) shows a representative TEM image of LIG flakes. HRTEM image in Figure 6.8 (b) shows the average lattice space of $3.4 \AA$ corresponds to the distance between two adjacent (002) planes in graphitic materials, in agreement with the XRD results (Figure 6.5). The formation the nano-shaped wrinkles observed could be attributed to thermal expansion caused by laser irradiation.
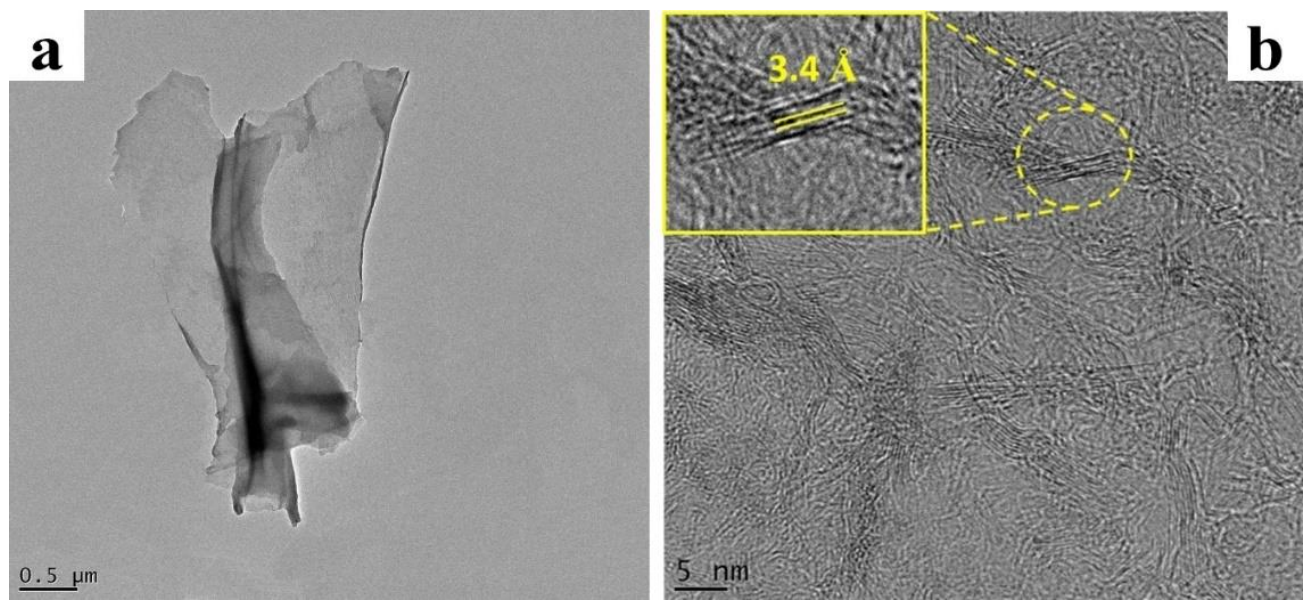

Figure 6.8 Transmission electron microscopy (TEM) images of LIG. (a) TEM image of LIG flakes. (b) High-resolution TEM (HRTEM) of LIG shows the average lattice space of $3.4 \AA$ corresponds to the (002) planes of graphitic materials.

XPS analysis showed that the carbon percentage of LIG was $97.91 \pm 0.5 \%$, oxygen percentage $1.96 \pm 0.3 \%$, and nitrogen percentage $0.64 \pm 0.03 \%$ (Figure 6.9 (a)). Highresolution XPS displayed two major peaks in the C1s spectrum (Figure 6.9 (b)), which can be assigned to the carbonyl $(\mathrm{C}=\mathrm{O})$ at $290.5 \mathrm{eV}$ and the $\mathrm{sp}^{2}$-hybridized carbon $(\mathrm{C}-\mathrm{C})$ at $284.5 \mathrm{eV}$. High-resolution O1s XPS spectrum of LIG showed two peaks, C-O at 533 $\mathrm{eV}$ and $\mathrm{C}=\mathrm{O}$ at $531.5 \mathrm{eV}$ (Figure 6.9 (c)). From the results, it can be concluded that LIG became partially oxidized during synthesis, since the thermal treatment was conducted in air, which led to the formation of oxygen containing surface functional groups, reflected 
in the observed $\zeta$ values. Finally, the high-resolution N1s XPS spectrum showed the C-N peak at $400.4 \mathrm{eV}$ (Figure $6.9(\mathrm{~d}))$.
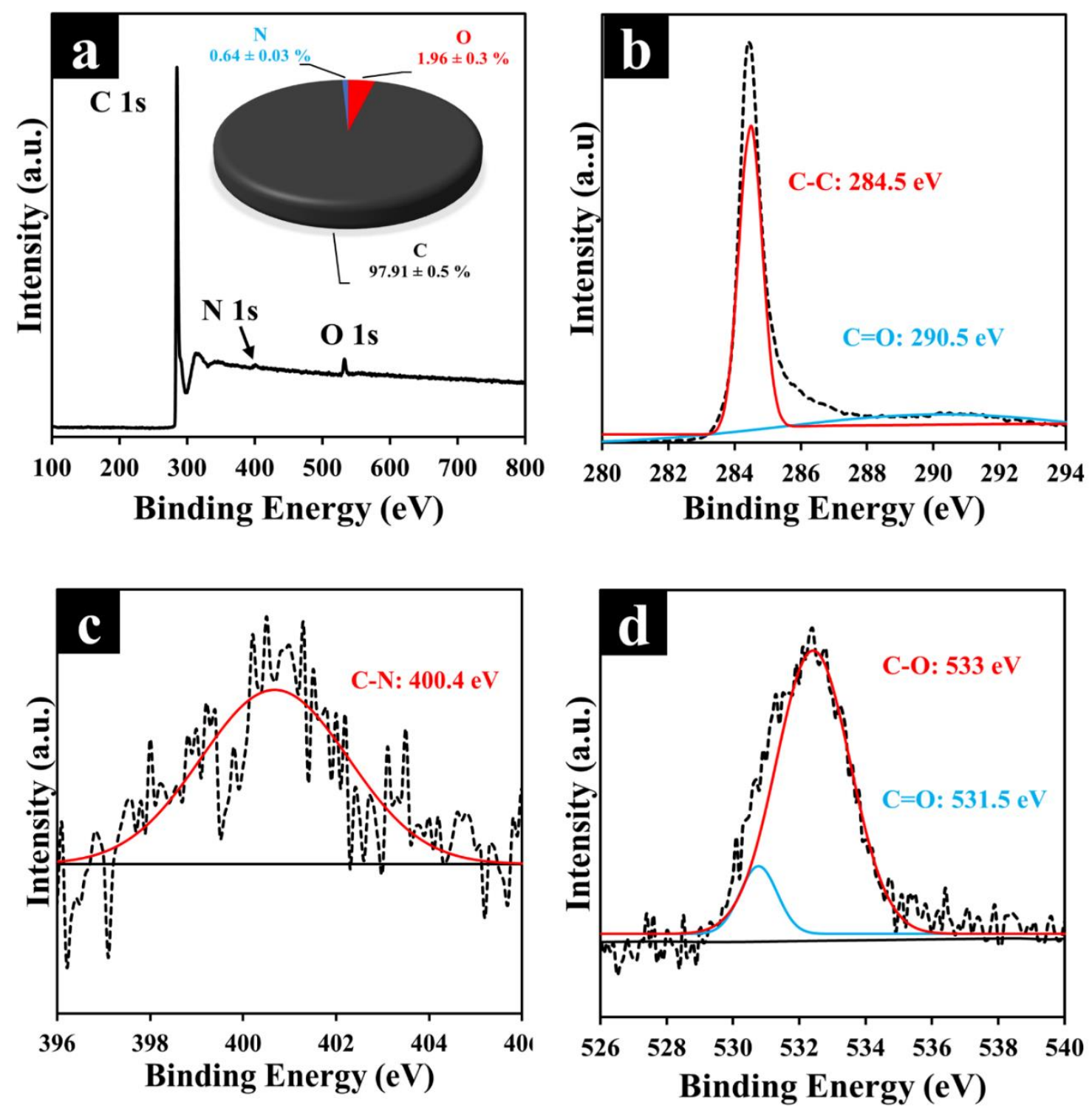

Figure 6.9 XPS spectra. (a) A wide scan XPS spectra of LIG. (b) High-resolution XPS of C1s peaks. (c) High-resolution XPS of N1s peak. (d) High-resolution XPS of O1s peak. 


\subsubsection{Adsorption kinetics}

Adsorption kinetics is an important characteristic that affects the overall process efficiency. According to Figure 6.10, ATZ adsorbed rapidly in the first $2 \mathrm{~h}$, and gradually reached apparent equilibrium after $48 \mathrm{~h}$. The mass of ATZ adsorbed at equilibrium by LIG was observed to be $10.3 \mathrm{mg}$ ATZ/g LIG, at initial ATZ concentration of $5 \mathrm{mg} / \mathrm{L}$ and pH 6. Approximately $90 \%$ of the ATZ initially present was adsorbed after $48 \mathrm{~h}$.

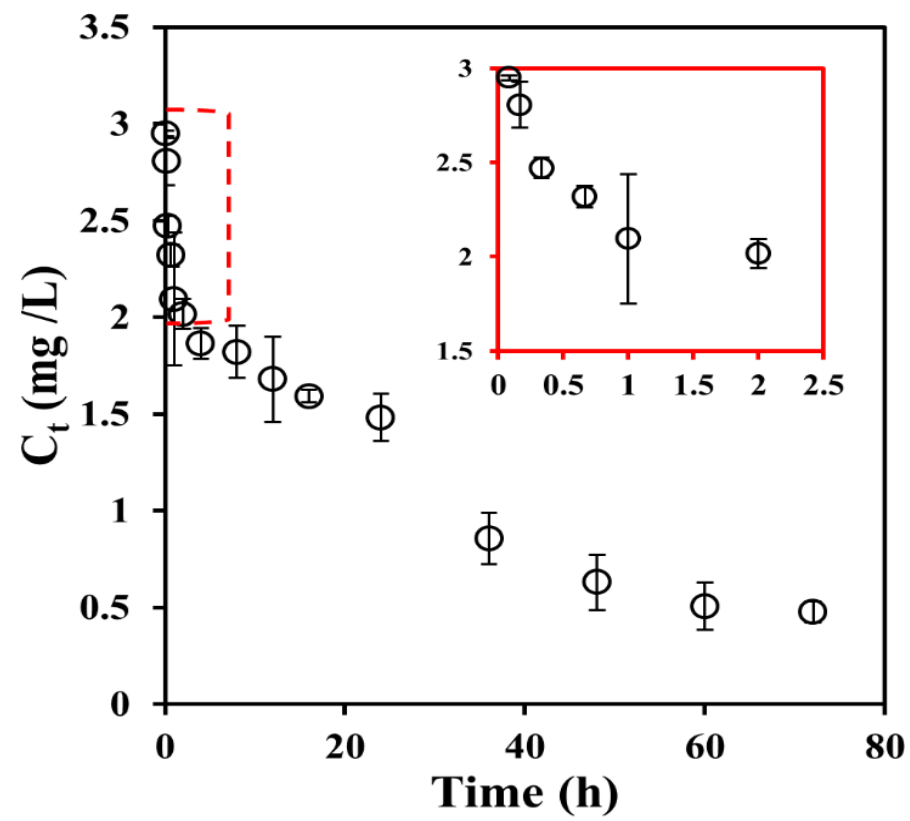

Figure 6.10 Kinetics of atrazine (ATZ) adsorption on laser induced graphitic material (LIG) (pH $=6.0, \mathrm{ATZ}$ concentration $=5 \mathrm{mg} / \mathrm{L}$, volume $=100 \mathrm{ml}, \mathrm{LIG}$ dosage $=40 \mathrm{mg}$, room temperature) .

The linear regression model for the adsorption kinetics is shown in Figure 6.11 (a) and (b). The pseudo second order model (Equation 6.5) provides a better fit to the experimental data than the pseudo first order model (Equation 6.4) with a coefficient of determination $\left(\mathrm{R}^{2}\right)$ value of 0.999 . Furthermore, the $q_{e, \text { experimental }}$ value was close to the $q_{e, \text { calculated }}$ value from Equation 6.5, indicating that the adsorption kinetics of ATZ by LIG can be better described by pseudo-second-order model, in agreement with previously 
reported studies of atrazine adsorption . Yue et al. [220] studied the adsorption kinetics of atrazine in three kinds of soils.
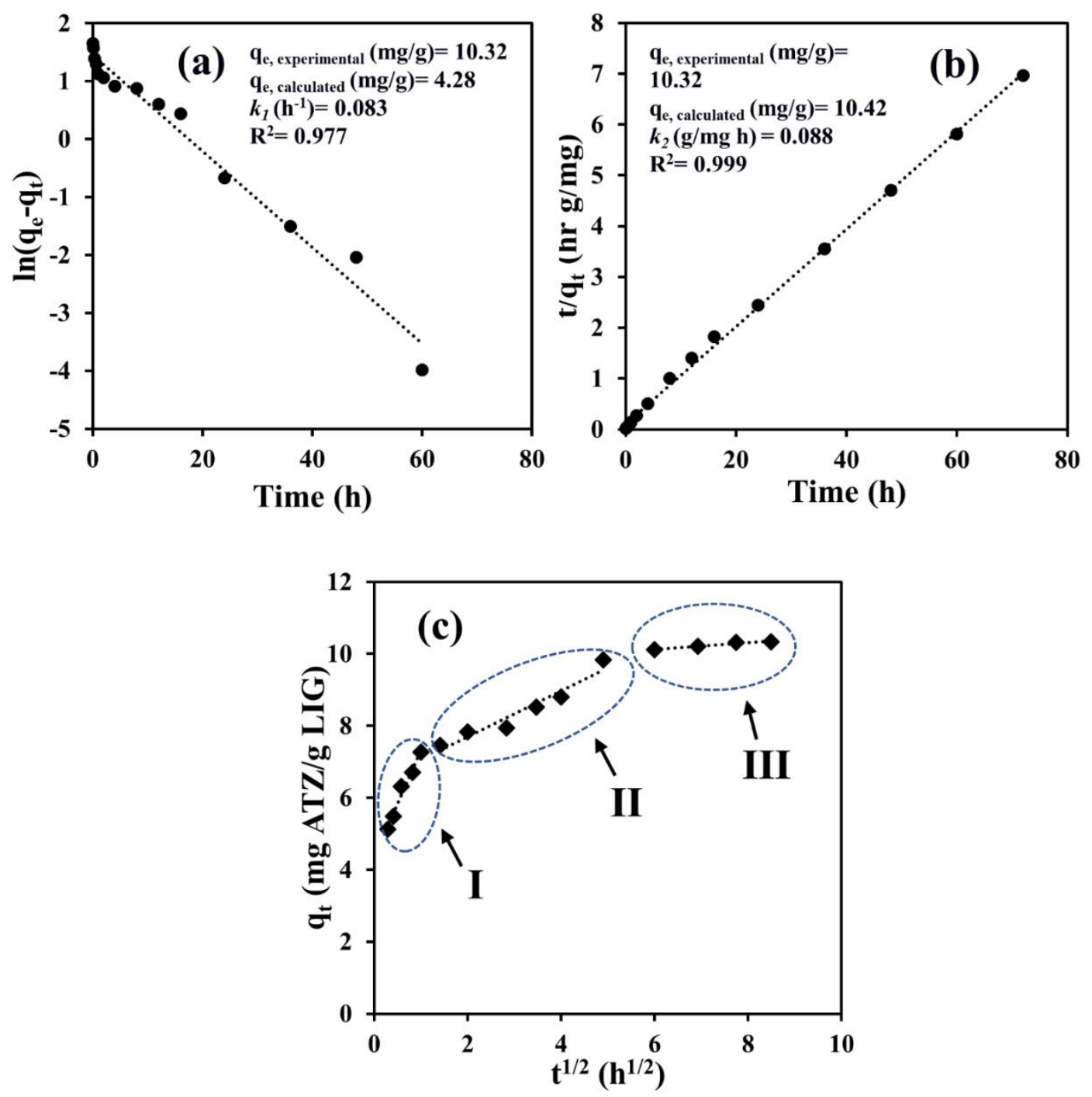

Figure 6.11 Linear regressions of kinetics plot obtained for atrazine (ATZ) removal by laser induced graphitic material (LIG) $(\mathrm{pH}=6.0$, ATZ concentration $=5 \mathrm{mg} / \mathrm{L}$, volume $=100 \mathrm{ml}$, LIG dosage $=40 \mathrm{mg}$ and room temperature): (a) Pseudo-first order model, (b) Pseudo-second order model, (c) Intraparticle diffusion model.

The results were satisfactorily described by a pseudo second order model, indicating that the rate-limiting step was chemical adsorption including valent forces through 
sharing or exchanging of electron [240]. Furthermore, the adsorption of atrazine on oxidized multiwalled carbon nanotubes (MWCNTs) was investigated by Chen et al. [217]. The regression results showed that pseudo second order model fit well the adsorption kinetics comparing to pseudo first order and intraparticle diffusion models. In order to determine whether intraparticle diffusion or film diffusion is the rate limiting step, the intraparticle diffusion model parameters were calculated (Table 6.2).

Table 6.2 Three phase parameters of the intraparticle diffusion kinetic model

\begin{tabular}{cccc}
\hline Phase & $\begin{array}{c}\boldsymbol{k}_{\boldsymbol{i}} \\
{\left[\mathbf{m g} /\left(\mathbf{g} \mathbf{~ h}^{1 / 2}\right)\right]}\end{array}$ & $\begin{array}{c}\boldsymbol{C} \\
(\mathbf{m g} / \mathbf{g})\end{array}$ & $\boldsymbol{R}^{\mathbf{2}}$ \\
\hline I & 2.96 & 4.35 & 0.97 \\
II & 0.64 & 6.42 & 0.93 \\
III & 0.09 & 9.56 & 0.96 \\
\hline
\end{tabular}

The model proposes that if the adsorption mechanism is controlled by intraparticle diffusion, then the plot of $q_{t}$ vs $t^{1 / 2}$ will be linear and intraparticle diffusion is the sole rate-limiting step when such a plot passes through the origin. However, when the plot of $q_{t} \mathrm{vs} t^{1 / 2}$ will be multi-linear, then the adsorption process is controlled by more than one mechanism. The multi-linear nature of the plot for ATZ adsorption on the LIG presented three phases (Figure 6.11 (c), Table 1). The initial steep phase $(0-1 \mathrm{~h})$ represented external mass transfer related to boundary layer effect (Figure 6.11 (c-I)), the second less steep phase (1-24 h) represented a gradual adsorption of ATZ where intraparticle diffusion within the pores is dominant (Figure 6.11 (c-II)), and the third phase (24-72 h) represented when equilibrium had been achieved (Figure 6.11 (c-III)). Since the intercepting lines do not pass through the origin, the intraparticle diffusion model is not 
the overall rate limiting step. Moreover, Chen et al. [217] investigated the adsorption of atrazine on MWCNTs and showed that the intraparticle diffusion was not the sole ratecontrolling step, which concurs with results of this study.

\subsubsection{Adsorption isotherms}

Adsorption isotherms were used to describe the partition of ATZ between the aqueous phase and the solid phase, in other words, the relationship between the amount of ATZ adsorbed $\left(q_{\mathrm{e}}\right)$ and the concentration remaining in the solution after the system has reached equilibrium $\left(C_{\mathrm{e}}\right)$. Additionally, isotherms can provide some insight about surface properties, the affinity of the adsorbate to the adsorbent, and adsorption mechanism, derived from the parameters of adsorption equilibrium models. For example, Freundlich isotherm gives an expression which determines the exponential distribution of active sites and surface heterogeneity. Additionally, Langmuir isotherm defines surface homogeneity and adsorption on perfectly smooth surfaces $[239,241]$.

Adsorption isotherms of ATZ in LIG are plotted in Figure 6.12 and Figure 6.13 (a). LType isotherm is characterized by the decrease in the adsorption at higher aqueous concentration of the compound, which indicates greater competition for adsorption sites that are limited as concentration in the liquid increases [242]. ATZ is a weakly basic molecule with $\mathrm{pK}_{\mathrm{b}}$ of 12.3 (Table 6.1). As shown in Figure 6.12, the solution $\mathrm{pH}$ had a strong impact on the adsorption of ATZ on LIG. At pH 3, where LIG was positively charged (Figure 6.3) and ATZ was protonated, the electrostatic repulsion between ATZ and LIG made ATZ adsorption unfavorable (Figure 6.12). ATZ exists solely as neutral molecules at higher $\mathrm{pH}$ (4-9) [243] and forces such as hydrophobic interactions and 
hydrogen bonding would be responsible of its adsorption on LIG. Chen et al. [217] has reported similar results for the adsorption of ATZ on MWCNTs. ATZ was adsorbed onto LIG surface as uncharged species at $\mathrm{pH}$ between 4 and 9, and subsequently, electrostatic interactions may not impact its adsorption from aqueous solution.

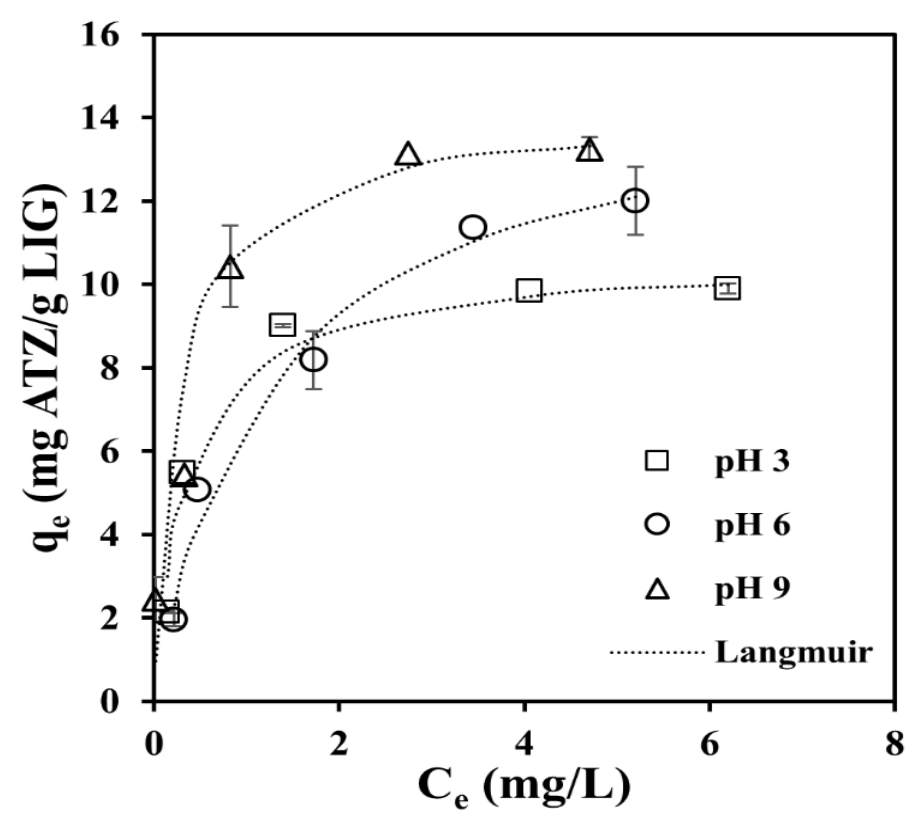

Figure 6.12. Adsorption isotherms of atrazine (ATZ) on laser induced graphitic material (LIG) at different $\mathrm{pH}$ fitted with Langmuir model $(\mathrm{pH}=3,6,9$, ATZ concentration= $1-10 \mathrm{mg} / \mathrm{L}$, volume= $50 \mathrm{ml}$, LIG dosage $=10 \mathrm{mg}$ and room temperature).

The influence of ionic strength on the adsorption of ATZ on LIG was studied and shown in Figure 6.13 (a). The highest adsorption capacity was found in the presence of $100 \mathrm{mM} \mathrm{NaNO}$ (18.7 mg ATZ/g LIG). This observation seems to suggest that the adsorption sites available for ATZ were influenced by the increase of IS; however, no significant difference was observed in present of $1 \mathrm{mM}$ and $10 \mathrm{mM} \mathrm{NaNO}_{3}$, which suggests the absence of IS effect at neutral $\mathrm{pH}$. The decrease in solubility of ATZ in salt solution, characterizing as 'salting out' effect explains this phenomenon. Salts ions can 
reduce the amount of water available to interact with ATZ, since they attract the polarizable water molecules around themselves [245,246]. This decrease in solubility resulted in an increase in hydrophobic interactions between ATZ and LIG, leading to an increase in the adsorption capacity.

The adsorption parameters of the Langmuir and Freundlich models are listed in Table 6.3. The Langmuir isotherm is used mainly for monolayer adsorption on homogeneous and perfectly smooth surfaces, while the Freundlich isotherm is used for surfaces with heterogeneous energy distribution. As shown in Figure. 9, Figure. 10(a) and Table 2, ATZ sorption data were well fitted by Langmuir model, indicating that the experimental results are compatible with a homogenous distribution of active sites on the LIG surface. The maximum amount of ATZ that could be adsorbed by the LIG is represented by the value of $q_{\max }$. Highest values of $q_{\max }$ were obtained for $\mathrm{pH} 9$ (15.0 mg ATZ/g LIG) and at the highest level of ionic strength tests, $100 \mathrm{mM} \mathrm{NaNO}_{3}(18.7 \mathrm{mg}$ ATZ/g LIG).

Table 6.3 Isotherm parameters for atrazine adsorption on LIG under different $\mathrm{pH}$ and ionic strength

\begin{tabular}{|c|c|c|c|c|c|c|}
\hline & \multicolumn{3}{|c|}{ pH } & \multicolumn{3}{|c|}{$\mathrm{NaNO}_{3}$} \\
\hline & 3 & 6 & 9 & $1 \mathrm{mM}$ & $10 \mathrm{mM}$ & $100 \mathrm{mM}$ \\
\hline Langmuir & & & & & & \\
\hline$q_{\max }(\mathrm{mg} / \mathrm{g})$ & 10.6 & 14.1 & 15.0 & 10.6 & 10.4 & 18.7 \\
\hline$K_{L}(\mathrm{~L} / \mathrm{mg})$ & 0.82 & 2.72 & 3.56 & 3.06 & 2.86 & 4.65 \\
\hline$R^{2}$ & 0.98 & 0.99 & 0.99 & 0.98 & 0.98 & 0.97 \\
\hline Freundlich & & & & & & \\
\hline $\begin{array}{l}K_{F} \\
{\left[(\mathrm{mg} / \mathrm{g})(\mathrm{mg} / \mathrm{L})^{-\mathrm{n}}\right]}\end{array}$ & 5.69 & 6.15 & 9.02 & 6.78 & 11.0 & 12.4 \\
\hline$n$ & 1.86 & 2.75 & 3.07 & 3.95 & 3.36 & 3.77 \\
\hline$R^{2}$ & 0.97 & 0.96 & 0.93 & 0.96 & 0.98 & 0.96 \\
\hline
\end{tabular}


Several interactions, such as van der Waals forces and hydrophobic effects [247,248], electrostatic interactions [249], hydrogen bonding [250] or $\pi-\pi$ interactions [251] have been reported to be implicated in atrazine adsorption on numerous adsorbents. The possible interactions of ATZ with LIG are shown in Figure 6.13 (b).

The high hydrogen-bonding potency of atrazine molecules was highlighted by Welhouse and Bleam [252]. In the triazine ring, hydrogen bonds can be formed with the ethylamino group, while the para nitrogen side chain can be a hydrogen-bond acceptor. Lupul et al. [218] found that an S-type isotherm would be obtained if ATZ adsorption were mainly controlled by hydrogen bond formation, while an L-type isotherm was characteristic for the activated carbon studied, which concurs with the results of this study. Another reason that hydrogen bonding is not the dominated mechanism in adsorption of ATZ on LIG is related to the very low percentage of oxygen $(1.96 \pm 0.31)$ found on LIG surface. We emphasize the importance of $\pi-\pi$ interactions between the heterocyclic ring of ATZ and the aromatic carbon of graphene layers in the LIG. Similar results were reported by Lupul et al. [218] for the adsorption of ATZ on activated carbon (AC). Since LIG is highly hydrophobic, with a contact angle of $138.4^{\circ}$ [233], hydrophobic interactions between ATZ and LIG cannot be eliminated. Polar nitrogen and oxygen functional groups on the carbon surface may reduce the accessibility of ATZ to adsorption sites because they act as water binding centers and ease the formation of water clusters [215]. 

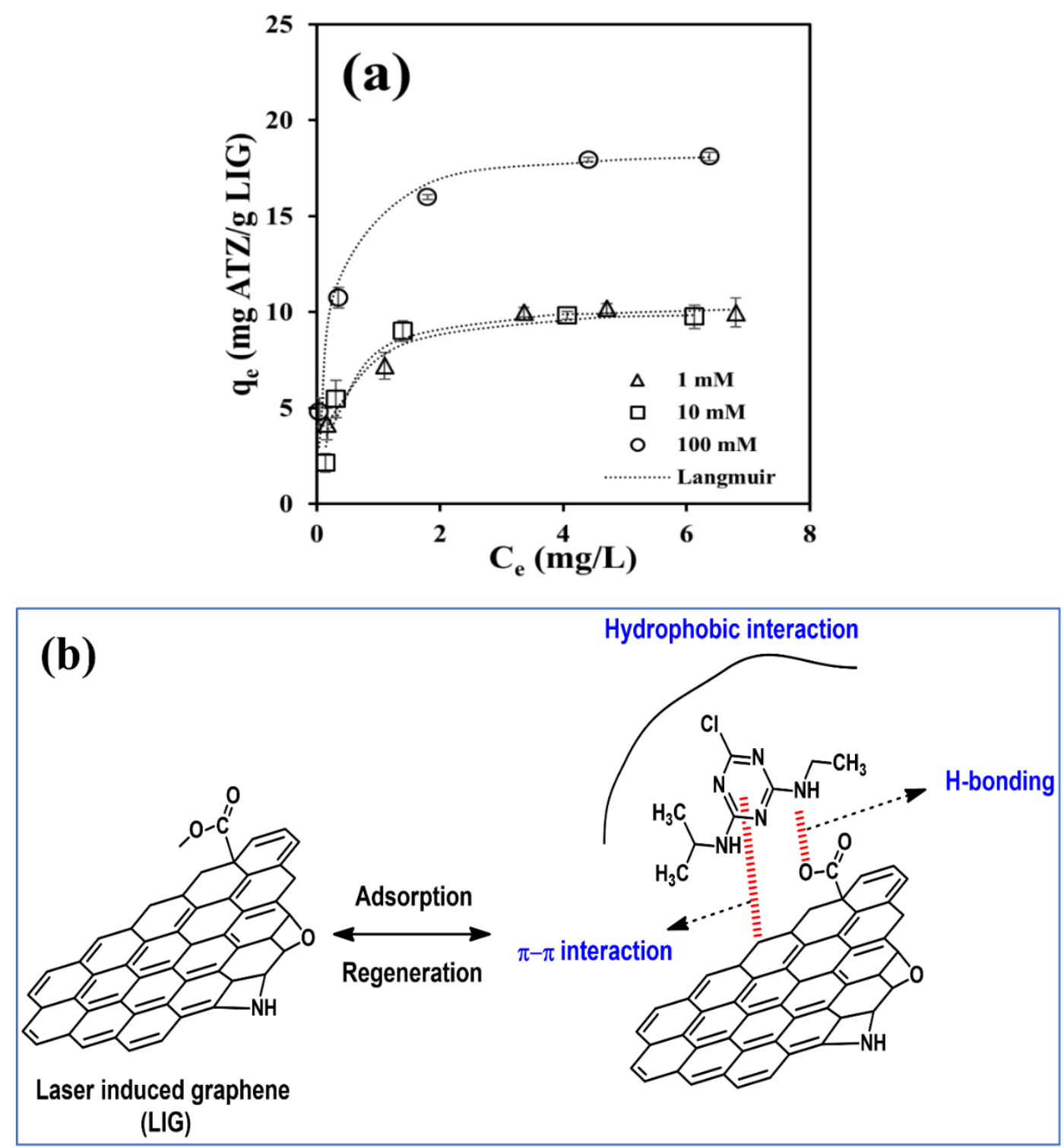

Figure 6.13 (a) Adsorption isotherms of atrazine (ATZ) on laser induced graphitic material (LIG) at different ionic strength fitted with Langmuir model (IS $=1,10,100 \mathrm{mM}, \mathrm{pH}=6, \mathrm{ATZ}$ concentration $=1-10 \mathrm{mg} / \mathrm{L}$, volume $=50 \mathrm{ml}, \mathrm{LIG}$ dosage $=10 \mathrm{mg}$ and room temperature). (b) Possible interactions of ATZ with LIG.

The advantage of LIG as adsorbent can be recognized from the comparison of its adsorption capacity with other proposed materials, including iron nanoparticles (INPs), 
activated carbon (AC), carbon nanotubes, fly ash, wood charcoal, activated peanut husk and banana peel-based sorbent (Table 6.4). Notably, LIG possessed a fairly high adsorption capacity (15.0 mg ATZ/g LIG) compared with many adsorbents listed in Table 3. For example, INPs [253], AC [215], MWCNTs [217] and granular carbon [242] removed ATZ with a maximum adsorption capacity of $0.011,8.51,10.5,5 \mathrm{mg} / \mathrm{g}$ respectively. CNTs and magnetic MWCNTs have shown higher adsorption capacity than LIG (Table 6.4); however, the toxicity of these materials should be considered. Different types of CNTs resulted toxic to zebrafish and mammalian cells [229-231]. A recent study by d'Amora et al. [232] showed that LIG does not exhibit toxicity and it does not interfere with zebrafish development, making it a promising platform for several environmental applications. 
Table 6.4 Comparison of adsorption capacity of different adsorbents for the removal of atrazine.

\begin{tabular}{|c|c|c|c|c|c|}
\hline Adsorbent & $\begin{array}{l}\text { Maximum } \\
\text { adsorption } \\
\text { capacity } \\
(\mathrm{mg} / \mathrm{g})\end{array}$ & Kinetic model & $\begin{array}{c}\text { Isotherm } \\
\text { model }\end{array}$ & pH & Reference \\
\hline $\begin{array}{l}\text { Iron nanoparticles } \\
\text { (INPs) }\end{array}$ & 0.011 & Pseudo-first-order & Freundlich & 7 & [253] \\
\hline $\begin{array}{l}\text { Conventional activated } \\
\text { carbons }\end{array}$ & 8.51 & $\begin{array}{l}\text { Pseudo-second- } \\
\text { order }\end{array}$ & Freundlich & 5 & [215] \\
\hline $\begin{array}{l}\text { Magnetic multi-walled } \\
\text { carbon nanotube } \\
(\text { MMWCNT) }\end{array}$ & 17.95 & $\begin{array}{l}\text { Pseudo-second- } \\
\text { order }\end{array}$ & Freundlich & 6 & [247] \\
\hline $\begin{array}{l}\text { Carbon nanotubes } \\
\text { (CNTs) }\end{array}$ & 31.37 & - & $\begin{array}{l}\text { Polanyi- } \\
\text { Manes }\end{array}$ & 7.8 & [254] \\
\hline $\begin{array}{l}\text { Activated carbon (AC)/ } \\
\mathrm{MgO/ZnO} \text { composite }\end{array}$ & 22.75 & $\begin{array}{l}\text { Pseudo-second- } \\
\text { order }\end{array}$ & Langmuir & 7 & [219] \\
\hline $\begin{array}{l}\text { Multiwalled carbon } \\
\text { nanotubes } \\
\text { (MWCNTs) }\end{array}$ & 10.50 & $\begin{array}{l}\text { Pseudo-second- } \\
\text { order }\end{array}$ & Polanyi-Manes & 6 & [217] \\
\hline $\begin{array}{l}\text { Banana peel-based } \\
\text { sorbent }\end{array}$ & 14 & - & Langmuir & $7-8.1$ & [255] \\
\hline Fly ash & 5.56 & - & Freundlich & 6.75 & [256] \\
\hline Green waste biochar & 0.435 & - & Freundlich & $<7$ & [257] \\
\hline Granular carbon & 5 & - & Langmuir & 6.85 & [242] \\
\hline Wood charcoal & 0.8 & & Freundlich & $5.8-6$ & [258] \\
\hline Rubber granules & 0.47 & & Freundlich & $5.8-6$ & [258] \\
\hline Activate peanut husk & 4.12 & $\begin{array}{l}\text { Pseudo-second- } \\
\text { order }\end{array}$ & Freundlich & $4-6$ & [259] \\
\hline $\begin{array}{l}\text { Fe3O4/reduced } \\
\text { graphene oxide (rGO) }\end{array}$ & 15 & - & - & 5 & [260] \\
\hline GO/nZVI & 4.93 & $\begin{array}{l}\text { Pseudo-second- } \\
\text { order }\end{array}$ & - & 6.7 & [261] \\
\hline $\begin{array}{l}\text { Laser induced } \\
\text { graphitic material } \\
\text { (LIG) }\end{array}$ & 15.0 & $\begin{array}{l}\text { Pseudo-second- } \\
\text { order }\end{array}$ & Langmuir & 9 & This study \\
\hline
\end{tabular}




\subsubsection{Adsorption of ATZ by LIG in the presence of HA}

The presence of $\mathrm{HA}$ in the aquatic environment introduces both hydrophilic and hydrophobic groups, enabling interactions with organic molecules. A negligible interaction between ATZ and HA was assumed based on the literature [262,263]. Atrazine is a small molecule (MW $=215.68 \mathrm{~g} / \mathrm{mol})$ with molecular dimensions of 0.96 $\mathrm{nm} \times 0.84 \mathrm{~nm} \times 0.3 \mathrm{~nm}$ (Table 6.1). Since most of the NOM molecules are larger than ATZ [264], the kinetic factors would ensure that the ATZ could diffuse into the mesopores of LIG before the larger HA molecules travel far enough to block pores [265]. In contrast to our results, a severe reduction in adsorption was observed for triazine-based pollutants by CNTs in the presence of NOM [266-268]. Surprisingly, the presence of HA has only slightly affected the adsorption of ATZ on the LIG, emphasizing the affinity of LIG to ATZ and the importance of hydrophobic interactions between them in the presence of HA. Whether the HA was initially equilibrated with the LIG, was added simultaneously or after adsorption of the ATZ, it had a pronounced effect on the outcome of the process (Figure 6.14). When the LIG was previously exposed to HA, the adsorption of ATZ was inhibited. A decrease of approximately $32 \%$ in ATZ adsorption capacity was noted. The adsorption capacity of HA was $7.2 \mathrm{mg} \mathrm{HA} / \mathrm{g} \mathrm{LIG}$, at pH 6 and initial concentration of $5 \mathrm{mg} / \mathrm{L}$. The decrease in ATZ adsorption is most likely due to the occupation of active sites by HA, reducing the number of those available for ATZ. An identical phenomenon was observed in the case of SWCNTs pre-loaded with NOM and exposed to ATZ, which caused a 95\% reduction in adsorption capacity $[262,269]$. The competition between NOM and SWCNTs was more severe than in the case of HA and 
LIG because of H-bonds formation between NOM and SWCNTs, resulting in site blockage and increased competition [262].

In post-HA introduction, i.e. HA introduced after the LIG-ATZ reached equilibrium, the adsorption capacity for ATZ was significantly higher than that in pre-introduction stage (Figure 6.14), although maximum adsorption capacity was still slightly higher in HA-free. This suggests that the adsorbed ATZ can be replaced by HA molecules with higher affinity to the LIG, and as a result, it released back into the solution.

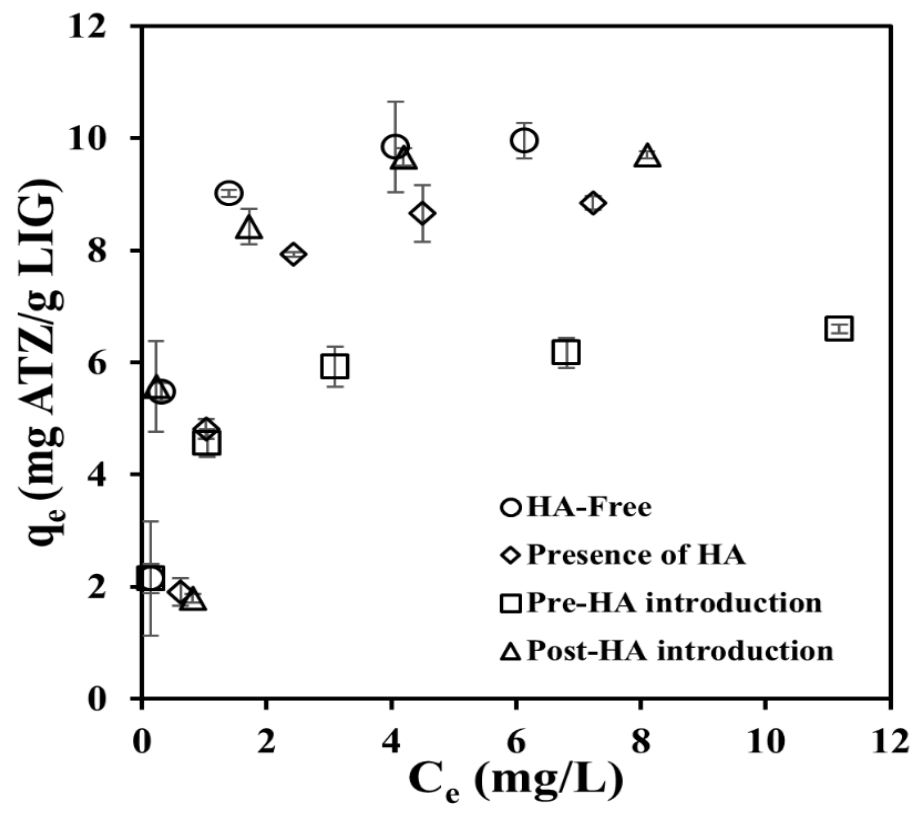

Figure 6.14 Adsorption isotherms of atrazine (ATZ) by laser induced graphitic material (LIG) in the presence of humic acid (HA) added at different stages. HA concentration in pre- and post-HA introduction was $5 \mathrm{mg} / \mathrm{L}$. All experiments preformed at $\mathrm{pH}=6.0$, ionic strength of $10 \mathrm{mM}$ and room temperature.

\subsubsection{Regeneration of LIG}

The safe disposal of the spent adsorbent is considered one of the main issues associated with the adsorption process [270]. Once the adsorbents have been exhausted, they can be disposed in a landfill or incinerated [271]. Landfill disposal of exhausted 
adsorbents with toxic contaminants can cause secondary pollution problems, such as underground water, soil, or surface water contamination through desorption process or natural leaching [272]. Therefore, the regeneration of the adsorbent is an essential aspect of the adsorption process both from environmental and economic point of view.

The regeneration and reuse of LIG was assessed by four consecutive adsorptiondesorption cycles (Figure 6.15). It can be seen that even after four cycles, LIG almost maintained its original adsorption ability. The results indicate that ethanol can effectively remove ATZ adsorbed on LIG and can repeatedly regenerate the exhausted LIG. Further investigation will be needed to fully assess the economic benefits and the engineering practicality of LIG regeneration with ethanol.

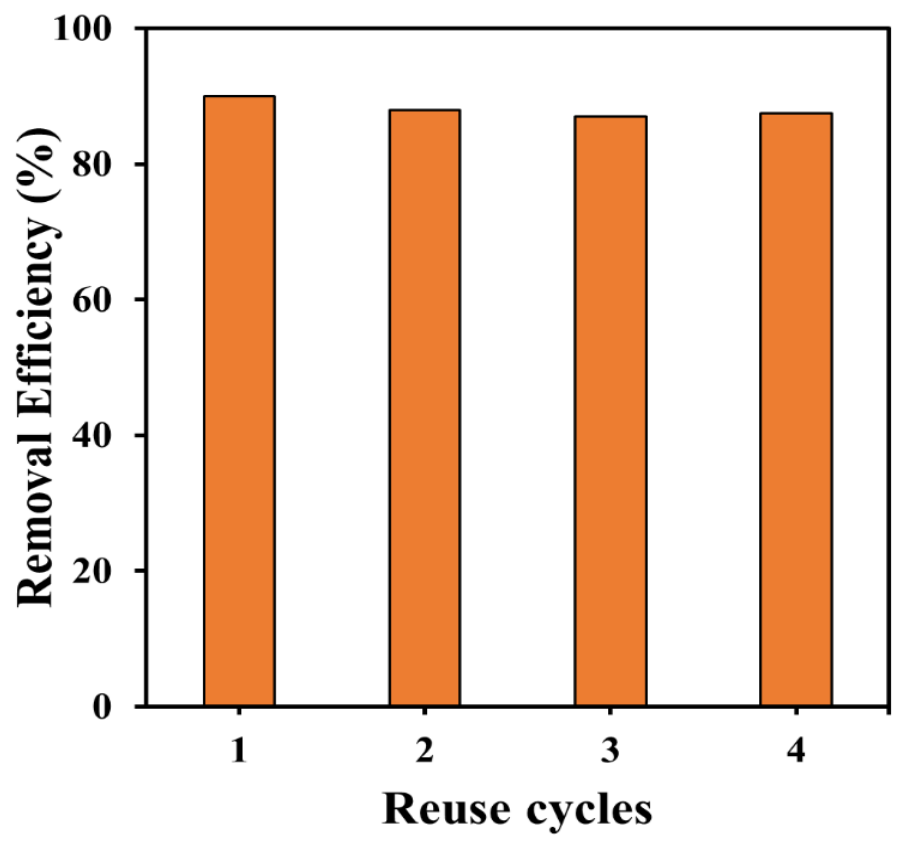

Figure 6.15 Adsorption of ATZ by LIG in consecutive cycles of use and regeneration (ATZ concentration $=5 \mathrm{mg} / \mathrm{L}, \mathrm{LIG}$ dose $=10 \mathrm{mg}$, agitating for $24 \mathrm{~h}$, room temperature) 


\subsection{Conclusion}

LIG was thoroughly investigated as an adsorbent for ATZ removal in water treatment. This graphite-based adsorbent was shown to be advantageous since it was manufactured under ambient conditions, in a one-step, scalable, reagent-free process synthesis from polyimide by surface irradiation with a $\mathrm{CO}_{2}$ laser. Adsorption of ATZ was influenced by solution $\mathrm{pH}$, but it was mostly insensitive to IS, except for solubility limitations at high IS levels. The presence of HA decreased the adsorption of ATZ on the LIG by $32 \%$, emphasizing the affinity of LIG to ATZ and the importance of hydrophobic interactions. The equilibrium data were well described by a Langmuir isotherm and the adsorption of ATZ by LIG followed a pseudo-second-order kinetics. The highest adsorption capacity was determined to be $15.0 \mathrm{mg}$ ATZ/g LIG, at pH 9, over performing many previously reported adsorbents. Furthermore, LIG could be easily regenerated by elution of the adsorbed ATZ with ethanol. In summary, the ease of fabrication, low cost, high adsorption capacity and reuse potential underscore the advantages of LIG as an effective adsorbent for the abatement of water pollution. 


\section{CHAPTER SEVEN}

\section{Conclusions and Future Work}

\subsection{Conclusions}

In the first part of this study, we have explored two types of carbon dots (plain and amine capped) and graphene oxide. Their surface charge, given by the zeta potential, and their hydrodynamic diameter in the suspension were investigated under a wide range of environmentally relevant conditions. The surface charge of both carbon dots varies with $\mathrm{pH}$ as expected. However, particle size remains fairly constant, always below $50 \mathrm{~nm}$. Although nanoparticles tend to aggregate because of their high surface energies, more strongly so for smaller particles as in the case of carbon dots, coatings (either engineered or adsorbed from the suspension media, e.g. NOM) may hinder aggregation. Aggregation is expected as a result of most typical concentrations of $\mathrm{Ca}^{2+}$ in surface waters regardless of the presence of NOM and under groundwater conditions, $\mathrm{Ca}^{2+}$ and $\mathrm{NOM}$ being key factors in nanoparticle stability, in contrast with our findings. If nanomaterials aggregate fast under natural water conditions and settle, they will impact mostly sediments and benthic organisms. We have shown in this work that carbon dots exhibit an opposite behavior, as they will most likely remain suspended in natural waters and will impact animals that inhabit the water column such as planktonic species, fish, and marine mammals. Moreover, high stability translates into excellent transport properties, which may lead to impacted ecosystems far from source of contamination. Presence of HA also led to a unique response, inducing aggregation due to bridging for low to moderate concentrations and followed by stabilization at higher levels, independently of surface 
charge modifications. Interestingly, both positively and negatively charged particles presented an increase in the magnitude of zeta potentials with HA concentration.

Fluorescence emission intensity declines were observed for water chemistry conditions at which specific adsorption of ions or HA was suspected, suggesting surface states as the origin of the PL. However, the adsorption of HA at the peak emission wavelength for the carbon dots may have also contributed to this observation. This phenomenon may limit the application of carbon dots in sensing of natural waters.

The result for graphene oxide study showed that divalent cations $\left(\mathrm{Ca}^{2+}\right)$ affect $\mathrm{GO}$ stability more noticeable than monovalent cations $\left(\mathrm{Na}^{+}\right)$because of the binding capacity of $\mathrm{Ca}^{2+}$ ions with functional groups of GO. Presence of NOM did not prevent $\mathrm{Ca}^{2+}$ from specifically adsorb at the surface, and therefore, repulsive electrostatic forces in the presence of NOM were comparable to those when only the electrolytes are present. Moreover, divalent cations adsorption on the particle surface occurred under conditions of presence and absence of NOM, as the molecules present similar functionality to that of the clean surface, resulting in $\mathrm{Ca}^{2+}$ promoted aggregation. However, this effect depended on the chemical functionality of the organic molecule, in particular on its concentration of carboxylic groups. GO aggregation can be accurately modeled with DLVO sphere-sphere interaction expressions, suggesting that the behavior of the aggregated GO is dominated by the crumpled, 3D sphere-like structure that it assumes in water suspension as it interacts with other solution constituents such as common cations. The results of this study highlighted the predominant effect of specific interactions as well as the chemical structure of adsorbed organics on GO stability in natural. 
In the second part of this study, laser induced graphene (LIG) was investigated as an efficient, scalable and green material for pollution abatement. Laser induced graphene/ceramic membrane (LIG/CM) asymmetric membrane was successfully fabricated for the first time, forming strongly bonded graphene layer on top of the ceramic support. Pure water and solvents with different polarities were used to understand the solvent flux behavior of LIG membrane. The permeate flux of solvents decreased in the following order: hexane $>$ toluene $>$ isopropanol $>$ water. Higher fluxes were observed for hexane and toluene (nonpolar solvents) than for isopropanol and water (polar solvents). All the liquids tested showed a consistent increase in flux with decreasing dipole moment and dielectric constant $(\varepsilon)$, representing solvent polarity, which might be the major parameters affecting solvent flow through LIG membrane. Filtration experiments showed that the LIG/CM had low rejection for particles ranged between $18.7 \pm 1.1 \mathrm{~nm}$ to $107 \pm 5.4 \mathrm{~nm}$; however, $90.22 \pm 8.3 \%$ rejection was achieved for particles with average size of $255.6 \pm$ $9.5 \mathrm{~nm}$. This result indicates that the fabricated LIG/CM had relatively large connecting pores, effective in separation processes, due to the sponge-like structure of graphene sheets. The fabricated membrane has potential for the separation of liquid phases based on polarity and particles in the submicron range size, making it a suitable candidate for many applications such as oil/water separation or solvent recovery from wastewater.

Finally, LIG was obtained from commercial polyimide film and extensively investigated as an adsorbent for atrazine (ATZ) removal in water treatment. This graphite-based adsorbent was shown to be advantageous since it was manufactured under ambient conditions, in a one-step, scalable, reagent-free process synthesis from polyimide by surface irradiation with a $\mathrm{CO}_{2}$ laser. Adsorption of ATZ was influenced by solution 
$\mathrm{pH}$, but it was mostly insensitive to ionic strength (IS), except for solubility limitations at high IS levels. The presence of humic acid (HA) decreased the adsorption of ATZ on the LIG by $32 \%$, emphasizing the affinity of LIG to ATZ and the importance of hydrophobic interactions. The highest adsorption capacity was determined to be $15.0 \mathrm{mg}$ ATZ/g LIG, at $\mathrm{pH}$ 9, over performing many previously reported adsorbents. Furthermore, LIG could be easily regenerated by elution of the adsorbed ATZ with ethanol. In summary, the ease of fabrication, low cost, high adsorption capacity and reuse potential underscore the advantages of LIG as an effective adsorbent for the abatement of water pollution.

\subsection{Future work}

The works have stimulated us to make the following suggestions for future work.

\subsubsection{Investigating the performance of the prepared LIG membrane for oil/water separation}

An asymmetric composite ceramic membrane was successfully fabricated, creating a LIG graphene coating as an effective separation layer on top of a porous ceramic support. The LIG was characterized as well as the parameters in the laser conversion process (laser power and raster speed). The prepared membrane will be used to separate oil/water emulsion. The laboratory made emulsion ( $1 \mathrm{~g} / \mathrm{L}$ of motor oil/ Span-80 surfactant in ultrapure water) will be used in this study. The membrane performance will be tested under different operating pressure. The fluxes and rejections for the coated and uncoated membranes will be calculated.

Furthermore, it is essential to investigate the physical stability of LIG/ceramic membrane. the membranes will be placed in $50 \mathrm{ml}$ beakers filled with ethanol and will be 
sonicated at a frequency of $40 \mathrm{kHz}$ for $30 \mathrm{~min}$. Oil/water separation will be performed before and after sonication.

\subsubsection{Investigating the removal efficiency of heavy metals from aqueous systems with thiol functionalized LIG membrane}

The removal of heavy metals, such as mercury, lead, thallium, cadmium, and arsenic, from natural waters has attracted considerable attention because of their adverse effects on environmental and human health. In this work, LIG membrane will be functionalized with thiol groups in order to create a novel membrane that has a high capacity and selectivity for heavy metals. Heavy metal ion removal efficiency under various operating conditions, e.g., transmembrane pressure (TMP), and solution $\mathrm{pH}$ will be quantified. In addition, the membrane will be tested for long duration filtration of solutions containing individual heavy metal and also for solution of mixed metals under optimized conditions. The membrane will be regenerated using in-situ chemical regeneration method. The membrane will be first thoroughly washed with distilled water for $10 \mathrm{~min}$. Then an acidic solution of $\mathrm{pH} 5.5$ (prepared by addition of $\mathrm{HCl}$ solution) will be passed over the membrane for regeneration. 


\section{Bibliography}

[1] S.N. Baker, G.A. Baker, Luminescent Carbon Nanodots: Emergent Nanolights, Angew. Chemie Int. Ed. 49 (2010) 6726-6744. doi:10.1002/anie.200906623.

[2] D. Li, R.B. Kaner, Graphene-Based Materials, Science (80-. ). 320 (2008) 1170 LP - 1171. http://science.sciencemag.org/content/320/5880/1170.abstract.

[3] C.N.R. Rao, A.K. Sood, K.S. Subrahmanyam, A. Govindaraj, Graphene: The New Two-Dimensional Nanomaterial, Angew. Chemie Int. Ed. 48 (2009) 7752-7777. doi:10.1002/anie.200901678.

[4] S. Qu, X. Wang, Q. Lu, X. Liu, L. Wang, A Biocompatible Fluorescent Ink Based on Water-Soluble Luminescent Carbon Nanodots, Angew. Chemie Int. Ed. 51 (2012) 12215-12218. doi:10.1002/anie.201206791.

[5] Y. Song, D. Feng, W. Shi, X. Li, H. Ma, Parallel comparative studies on the toxic effects of unmodified CdTe quantum dots, gold nanoparticles, and carbon nanodots on live cells as well as green gram sprouts, Talanta. 116 (2013) 237-244. doi:https://doi.org/10.1016/j.talanta.2013.05.022.

[6] P.-C. Hsu, P.-C. Chen, C.-M. Ou, H.-Y. Chang, H.-T. Chang, Extremely high inhibition activity of photoluminescent carbon nanodots toward cancer cells, J. Mater. Chem. B. 1 (2013) 1774-1781. doi:10.1039/C3TB00545C.

[7] A. Bianco, Graphene: Safe or Toxic? The Two Faces of the Medal, Angew. Chemie Int. Ed. 52 (2013) 4986-4997. doi:10.1002/anie.201209099.

[8] M.X. Gao, C.F. Liu, Z.L. Wu, Q.L. Zeng, X.X. Yang, W.B. Wu, Y.F. Li, C.Z. Huang, A surfactant-assisted redox hydrothermal route to prepare highly photoluminescent carbon quantum dots with aggregation-induced emission enhancement properties, Chem. Commun. 49 (2013) 8015-8017. doi:10.1039/C3CC44624G.

[9] I. Chowdhury, M.C. Duch, N.D. Mansukhani, M.C. Hersam, D. Bouchard, Colloidal Properties and Stability of Graphene Oxide Nanomaterials in the Aquatic Environment, Environ. Sci. Technol. 47 (2013) 6288-6296. doi:10.1021/es400483k.

[10] W.Z. Teo, M. Pumera, Graphene Oxides: Transformations in Natural Waters over a Period of Three Months, Chempluschem. 79 (2014) 844-849. doi:10.1002/cplu.201400033.

[11] L.-J. Liang, T. Wu, Y. Kang, Q. Wang, Dispersion of Graphene Sheets in Aqueous Solution by Oligodeoxynucleotides, ChemPhysChem. 14 (2013) 1626-1632. 
doi:10.1002/cphc.201201084.

[12] M. Lotya, P.J. King, U. Khan, S. De, J.N. Coleman, High-Concentration, Surfactant-Stabilized Graphene Dispersions, ACS Nano. 4 (2010) 3155-3162. doi:10.1021/nn1005304.

[13] N.B. Saleh, L.D. Pfefferle, M. Elimelech, Influence of Biomacromolecules and Humic Acid on the Aggregation Kinetics of Single-Walled Carbon Nanotubes, Environ. Sci. Technol. 44 (2010) 2412-2418. doi:10.1021/es903059t.

[14] J.D. Lanphere, C.J. Luth, S.L. Walker, Effects of Solution Chemistry on the Transport of Graphene Oxide in Saturated Porous Media, Environ. Sci. Technol. 47 (2013) 4255-4261. doi:10.1021/es400138c.

[15] K.Y. Yoon, S.J. An, Y. Chen, J.H. Lee, S.L. Bryant, R.S. Ruoff, C. Huh, K.P. Johnston, Graphene oxide nanoplatelet dispersions in concentrated $\mathrm{NaCl}$ and stabilization of oil/water emulsions, J. Colloid Interface Sci. 403 (2013) 1-6. doi:https://doi.org/10.1016/j.jcis.2013.03.012.

[16] J. Luo, L.J. Cote, V.C. Tung, A.T.L. Tan, P.E. Goins, J. Wu, J. Huang, Graphene Oxide Nanocolloids, J. Am. Chem. Soc. 132 (2010) 17667-17669. doi:10.1021/ja1078943.

[17] A.M. Dimiev, L.B. Alemany, J.M. Tour, Graphene Oxide. Origin of Acidity, Its Instability in Water, and a New Dynamic Structural Model, ACS Nano. 7 (2013) 576-588. doi:10.1021/nn3047378.

[18] W.S. Hummers, R.E. Offeman, Preparation of Graphitic Oxide, J. Am. Chem. Soc. 80 (1958) 1339. doi:10.1021/ja01539a017.

[19] J. Chen, B. Yao, C. Li, G. Shi, An improved Hummers method for eco-friendly synthesis of graphene oxide, Carbon N. Y. 64 (2013) 225-229. doi:https://doi.org/10.1016/j.carbon.2013.07.055.

[20] D.C. Marcano, D. V Kosynkin, J.M. Berlin, A. Sinitskii, Z. Sun, A. Slesarev, L.B. Alemany, W. Lu, J.M. Tour, Improved Synthesis of Graphene Oxide, ACS Nano. 4 (2010) 4806-4814. doi:10.1021/nn1006368.

[21] J. Lin, Z. Peng, Y. Liu, F. Ruiz-Zepeda, R. Ye, E.L.G. Samuel, M.J. Yacaman, B.I. Yakobson, J.M. Tour, Laser-induced porous graphene films from commercial polymers, Nat. Commun. 5 (2014) 5714. http://dx.doi.org/10.1038/ncomms6714.

[22] L. Li, J. Zhang, Z. Peng, Y. Li, C. Gao, Y. Ji, R. Ye, N.D. Kim, Q. Zhong, Y. Yang, H. Fei, G. Ruan, J.M. Tour, High-Performance Pseudocapacitive Microsupercapacitors from Laser-Induced Graphene, Adv. Mater. 28 (2016) 838845. doi:10.1002/adma.201503333. 
[23] S.P. Singh, Y. Li, A. Be'er, Y. Oren, J.M. Tour, C.J. Arnusch, Laser-Induced Graphene Layers and Electrodes Prevents Microbial Fouling and Exerts Antimicrobial Action, ACS Appl. Mater. Interfaces. 9 (2017) 18238-18247. doi:10.1021/acsami.7b04863.

[24] K. Rathinam, S.P. Singh, Y. Li, R. Kasher, J.M. Tour, C.J. Arnusch, Polyimide derived laser-induced graphene as adsorbent for cationic and anionic dyes, Carbon N. Y. 124 (2017) 515-524. doi:https://doi.org/10.1016/j.carbon.2017.08.079.

[25] A.L. Lavoisier, Oeuvres: Traité élémentaire de chimie, Imprimerie impériale, 1864.

[26] J.W. Patrick, Porosity in Carbons: Characterization and Applications, 1st ed., John Wiley \& Sons, Ltd, 1994.

[27] W.I.F. David, R.M. Ibberson, J.C. Matthewman, K. Prassides, T.J.S. Dennis, J.P. Hare, H.W. Kroto, R. Taylor, D.R.M. Walton, Crystal structure and bonding of ordered C60, Nature. 353 (1991) 147. http://dx.doi.org/10.1038/353147a0.

[28] H.W. Kroto, J.R. Heath, S.C. O’Brien, R.F. Curl, R.E. Smalley, C60:

Buckminsterfullerene, Nature. 318 (1985) 162.

http://dx.doi.org/10.1038/318162a0.

[29] S. Iijima, Helical microtubules of graphitic carbon, Nature. 354 (1991) 56. http://dx.doi.org/10.1038/354056a0.

[30] A.K. Geim, K.S. Novoselov, The rise of graphene, Nat. Mater. 6 (2007) 183. http://dx.doi.org/10.1038/nmat1849.

[31] K.S. Novoselov, A.K. Geim, S. V Morozov, D. Jiang, Y. Zhang, S. V Dubonos, I. V Grigorieva, A.A. Firsov, Electric Field Effect in Atomically Thin Carbon Films, Science (80-. ). 306 (2004) 666 LP - 669. http://science.sciencemag.org/content/306/5696/666.abstract.

[32] D.R. Dreyer, S. Park, C.W. Bielawski, R.S. Ruoff, The chemistry of graphene oxide, Chem. Soc. Rev. 39 (2010) 228-240. doi:10.1039/B917103G.

[33] X. Han, S. Li, Z. Peng, A.O. Al-Yuobi, A.S. Omar Bashammakh, M.S. ElShahawi, R.M. Leblanc, Interactions between Carbon Nanomaterials and Biomolecules, J. Oleo Sci. 65 (2016) 1-7. doi:10.5650/jos.ess15248.

[34] Z. Peng, E.H. Miyanji, Y. Zhou, J. Pardo, S.D. Hettiarachchi, S. Li, P.L. Blackwelder, I. Skromne, R.M. Leblanc, Carbon dots: promising biomaterials for bone-specific imaging and drug delivery, Nanoscale. 9 (2017) 17533-17543. doi:10.1039/C7NR05731H. 
[35] R. Sengupta, M. Bhattacharya, S. Bandyopadhyay, A.K. Bhowmick, A review on the mechanical and electrical properties of graphite and modified graphite reinforced polymer composites, Prog. Polym. Sci. 36 (2011) 638-670. doi:https://doi.org/10.1016/j.progpolymsci.2010.11.003.

[36] V. Singh, D. Joung, L. Zhai, S. Das, S.I. Khondaker, S. Seal, Graphene based materials: Past, present and future, Prog. Mater. Sci. 56 (2011) 1178-1271. doi:https://doi.org/10.1016/j.pmatsci.2011.03.003.

[37] A. Dato, Z. Lee, K.-J. Jeon, R. Erni, V. Radmilovic, T.J. Richardson, M. Frenklach, Clean and highly ordered graphene synthesized in the gas phase, Chem. Commun. (2009) 6095-6097. doi:10.1039/B911395A.

[38] T. Kuila, S. Bose, A.K. Mishra, P. Khanra, N.H. Kim, J.H. Lee, Chemical functionalization of graphene and its applications, Prog. Mater. Sci. 57 (2012) 1061-1105. doi:https://doi.org/10.1016/j.pmatsci.2012.03.002.

[39] Y. Hernandez, V. Nicolosi, M. Lotya, F.M. Blighe, Z. Sun, S. De, I.T. McGovern, B. Holland, M. Byrne, Y.K. Gun'Ko, J.J. Boland, P. Niraj, G. Duesberg, S. Krishnamurthy, R. Goodhue, J. Hutchison, V. Scardaci, A.C. Ferrari, J.N. Coleman, High-yield production of graphene by liquid-phase exfoliation of graphite, Nat. Nanotechnol. 3 (2008) 563. http://dx.doi.org/10.1038/nnano.2008.215.

[40] M.J. Allen, V.C. Tung, R.B. Kaner, Honeycomb Carbon: A Review of Graphene, Chem. Rev. 110 (2010) 132-145. doi:10.1021/cr900070d.

[41] Q. Zheng, Z. Li, J. Yang, J.-K. Kim, Graphene oxide-based transparent conductive films, Prog. Mater. Sci. 64 (2014) 200-247. doi:https://doi.org/10.1016/j.pmatsci.2014.03.004.

[42] C. Gómez-Navarro, R.T. Weitz, A.M. Bittner, M. Scolari, A. Mews, M. Burghard, K. Kern, Electronic Transport Properties of Individual Chemically Reduced Graphene Oxide Sheets, Nano Lett. 7 (2007) 3499-3503. doi:10.1021/n1072090c.

[43] H.A. Becerril, J. Mao, Z. Liu, R.M. Stoltenberg, Z. Bao, Y. Chen, Evaluation of Solution-Processed Reduced Graphene Oxide Films as Transparent Conductors, ACS Nano. 2 (2008) 463-470. doi:10.1021/nn700375n.

[44] H.-J. Shin, K.K. Kim, A. Benayad, S.-M. Yoon, H.K. Park, I.-S. Jung, M.H. Jin, H.-K. Jeong, J.M. Kim, J.-Y. Choi, Y.H. Lee, Efficient Reduction of Graphite Oxide by Sodium Borohydride and Its Effect on Electrical Conductance, Adv. Funct. Mater. 19 (2009) 1987-1992. doi:10.1002/adfm.200900167.

[45] M.J. McAllister, J.-L. Li, D.H. Adamson, H.C. Schniepp, A.A. Abdala, J. Liu, M. 
Herrera-Alonso, D.L. Milius, R. Car, R.K. Prud'homme, I.A. Aksay, Single Sheet Functionalized Graphene by Oxidation and Thermal Expansion of Graphite, Chem. Mater. 19 (2007) 4396-4404. doi:10.1021/cm0630800.

[46] O.C. Compton, B. Jain, D.A. Dikin, A. Abouimrane, K. Amine, S.T. Nguyen, Chemically Active Reduced Graphene Oxide with Tunable C/O Ratios, ACS Nano. 5 (2011) 4380-4391. doi:10.1021/nn1030725.

[47] W. Gao, L.B. Alemany, L. Ci, P.M. Ajayan, New insights into the structure and reduction of graphite oxide, Nat. Chem. 1 (2009) 403. http://dx.doi.org/10.1038/nchem.281.

[48] B.C. Brodie, XIII. On the atomic weight of graphite, Philos. Trans. R. Soc. London . 149 (1859) 249-259. doi:10.1098/rstl.1859.0013.

[49] R.J. Young, I.A. Kinloch, L. Gong, K.S. Novoselov, The mechanics of graphene nanocomposites: A review, Compos. Sci. Technol. 72 (2012) 1459-1476. doi:https://doi.org/10.1016/j.compscitech.2012.05.005.

[50] L. Staudenmaier, Verfahren zur Darstellung der Graphitsäure, Berichte Der Dtsch. Chem. Gesellschaft. 31 (2018) 1481-1487. doi:10.1002/cber.18980310237.

[51] B. Garg, T. Bisht, Y.-C. Ling, Graphene-Based Nanomaterials as Heterogeneous Acid Catalysts: A Comprehensive Perspective, Mol. . 19 (2014). doi:10.3390/molecules190914582.

[52] M.S. Mauter, M. Elimelech, Environmental Applications of Carbon-Based Nanomaterials, Environ. Sci. Technol. 42 (2008) 5843-5859. doi:10.1021/es8006904.

[53] K. Scida, P.W. Stege, G. Haby, G.A. Messina, C.D. García, Recent applications of carbon-based nanomaterials in analytical chemistry: Critical review, Anal. Chim. Acta. 691 (2011) 6-17. doi:https://doi.org/10.1016/j.aca.2011.02.025.

[54] B.-T. Zhang, X. Zheng, H.-F. Li, J.-M. Lin, Application of carbon-based nanomaterials in sample preparation: A review, Anal. Chim. Acta. 784 (2013) 117. doi:https://doi.org/10.1016/j.aca.2013.03.054.

[55] Y. Shen, Q. Fang, B. Chen, Environmental Applications of Three-Dimensional Graphene-Based Macrostructures: Adsorption, Transformation, and Detection, Environ. Sci. Technol. 49 (2015) 67-84. doi:10.1021/es504421y.

[56] S. Das, S. Singh, V. Singh, D. Joung, J.M. Dowding, D. Reid, J. Anderson, L. Zhai, S.I. Khondaker, W.T. Self, S. Seal, Oxygenated Functional Group Density on Graphene Oxide: Its Effect on Cell Toxicity, Part. Part. Syst. Charact. 30 (2013) 148-157. doi:10.1002/ppsc.201200066. 
[57] S. Liu, T.H. Zeng, M. Hofmann, E. Burcombe, J. Wei, R. Jiang, J. Kong, Y. Chen, Antibacterial Activity of Graphite, Graphite Oxide, Graphene Oxide, and Reduced Graphene Oxide: Membrane and Oxidative Stress, ACS Nano. 5 (2011) 69716980. doi:10.1021/nn202451x.

[58] X. Hu, M. Zhou, Q. Zhou, Ambient Water and Visible-Light Irradiation Drive Changes in Graphene Morphology, Structure, Surface Chemistry, Aggregation, and Toxicity, Environ. Sci. Technol. 49 (2015) 3410-3418. doi:10.1021/es503003y.

[59] O. Akhavan, E. Ghaderi, Toxicity of Graphene and Graphene Oxide Nanowalls Against Bacteria, ACS Nano. 4 (2010) 5731-5736. doi:10.1021/nn101390x.

[60] K.-H. Liao, Y.-S. Lin, C.W. Macosko, C.L. Haynes, Cytotoxicity of Graphene Oxide and Graphene in Human Erythrocytes and Skin Fibroblasts, ACS Appl. Mater. Interfaces. 3 (2011) 2607-2615. doi:10.1021/am200428v.

[61] K. Lu, Q. Huang, P. Wang, L. Mao, Physicochemical Changes of Few-Layer Graphene in Peroxidase-Catalyzed Reactions: Characterization and Potential Ecological Effects, Environ. Sci. Technol. 49 (2015) 8558-8565. doi:10.1021/acs.est.5b02261.

[62] M.C. Duch, G.R.S. Budinger, Y.T. Liang, S. Soberanes, D. Urich, S.E. Chiarella, L.A. Campochiaro, A. Gonzalez, N.S. Chandel, M.C. Hersam, G.M. Mutlu, Minimizing Oxidation and Stable Nanoscale Dispersion Improves the Biocompatibility of Graphene in the Lung, Nano Lett. 11 (2011) 5201-5207. doi:10.1021/nl202515a.

[63] K. Lategan, H. Alghadi, M. Bayati, F.M. de Cortalezzi, E. Pool, Effects of Graphene Oxide Nanoparticles on the Immune System Biomarkers Produced by RAW 264.7 and Human Whole Blood Cell Cultures, Nanomater. . 8 (2018). doi:10.3390/nano8020125.

[64] X. Ren, J. Li, C. Chen, Y. Gao, D. Chen, M. Su, A. Alsaedi, T. Hayat, Graphene analogues in aquatic environments and porous media: dispersion, aggregation, deposition and transformation, Environ. Sci. Nano. 5 (2018) 1298-1340. doi:10.1039/C7EN01258F.

[65] I. Chowdhury, N.D. Mansukhani, L.M. Guiney, M.C. Hersam, D. Bouchard, Aggregation and Stability of Reduced Graphene Oxide: Complex Roles of Divalent Cations, pH, and Natural Organic Matter, Environ. Sci. Technol. 49 (2015) 10886-10893. doi:10.1021/acs.est.5b01866.

[66] C.-J. Shih, S. Lin, R. Sharma, M.S. Strano, D. Blankschtein, Understanding the pH-Dependent Behavior of Graphene Oxide Aqueous Solutions: A Comparative 
Experimental and Molecular Dynamics Simulation Study, Langmuir. 28 (2012) 235-241. doi:10.1021/la203607w.

[67] T. Taniguchi, S. Kurihara, H. Tateishi, K. Hatakeyama, M. Koinuma, H. Yokoi, M. Hara, H. Ishikawa, Y. Matsumoto, $\mathrm{pH}$-driven, reversible epoxy ring opening/closing in graphene oxide, Carbon N. Y. 84 (2015) 560-566. doi:https://doi.org/10.1016/j.carbon.2014.12.054.

[68] Y.-T. Shieh, G.-L. Liu, H.-H. Wu, C.-C. Lee, Effects of polarity and pH on the solubility of acid-treated carbon nanotubes in different media, Carbon N. Y. 45 (2007) 1880-1890. doi:https://doi.org/10.1016/j.carbon.2007.04.028.

[69] H. Tang, Y. Zhao, X. Yang, D. Liu, P. Shao, Z. Zhu, S. Shan, F. Cui, B. Xing, New Insight into the Aggregation of Graphene Oxide Using Molecular Dynamics Simulations and Extended Derjaguin-Landau-Verwey-Overbeek Theory, Environ. Sci. Technol. 51 (2017) 9674-9682. doi:10.1021/acs.est.7b01668.

[70] L. Wu, L. Liu, B. Gao, R. Muñoz-Carpena, M. Zhang, H. Chen, Z. Zhou, H. Wang, Aggregation Kinetics of Graphene Oxides in Aqueous Solutions: Experiments, Mechanisms, and Modeling, Langmuir. 29 (2013) 15174-15181. doi:10.1021/la404134x.

[71] X. Ren, Q. Wu, H. Xu, D. Shao, X. Tan, W. Shi, C. Chen, J. Li, Z. Chai, T. Hayat, $X$. Wang, New Insight into GO, Cadmium(II), Phosphate Interaction and Its Role in GO Colloidal Behavior, Environ. Sci. Technol. 50 (2016) 9361-9369. doi:10.1021/acs.est.6b02934.

[72] Y. Qi, T. Xia, Y. Li, L. Duan, W. Chen, Colloidal stability of reduced graphene oxide materials prepared using different reducing agents, Environ. Sci. Nano. 3 (2016) 1062-1071. doi:10.1039/C6EN00174B.

[73] B. Konkena, S. Vasudevan, Covalently Linked, Water-Dispersible, Cyclodextrin: Reduced-Graphene Oxide Sheets, Langmuir. 28 (2012) 12432-12437. doi:10.1021/la3020783.

[74] Y. Gao, X. Ren, X. Tan, T. Hayat, A. Alsaedi, C. Chen, Insights into key factors controlling GO stability in natural surface waters, J. Hazard. Mater. 335 (2017) 56-65. doi:https://doi.org/10.1016/j.jhazmat.2017.04.027.

[75] J.D. Lanphere, B. Rogers, C. Luth, C.H. Bolster, S.L. Walker, Stability and Transport of Graphene Oxide Nanoparticles in Groundwater and Surface Water, Environ. Eng. Sci. 31 (2014) 350-359. doi:10.1089/ees.2013.0392.

[76] B. Mukherjee, J.W. Weaver, Aggregation and Charge Behavior of Metallic and Nonmetallic Nanoparticles in the Presence of Competing Similarly-Charged 
Inorganic Ions, Environ. Sci. Technol. 44 (2010) 3332-3338.

doi:10.1021/es903456e.

[77] J. Brant, H. Lecoanet, M. Hotze, M. Wiesner, Comparison of Electrokinetic Properties of Colloidal Fullerenes (n-C60) Formed Using Two Procedures, Environ. Sci. Technol. 39 (2005) 6343-6351. doi:10.1021/es050090d.

[78] T. Hartono, S. Wang, Q. Ma, Z. Zhu, Layer structured graphite oxide as a novel adsorbent for humic acid removal from aqueous solution, J. Colloid Interface Sci. 333 (2009) 114-119. doi:https://doi.org/10.1016/j.jcis.2009.02.005.

[79] S. Wang, H. Sun, H.M. Ang, M.O. Tadé, Adsorptive remediation of environmental pollutants using novel graphene-based nanomaterials, Chem. Eng. J. 226 (2013) 336-347. doi:https://doi.org/10.1016/j.cej.2013.04.070.

[80] Y. Jiang, R. Raliya, P. Liao, P. Biswas, J.D. Fortner, Graphene oxides in water: assessing stability as a function of material and natural organic matter properties, Environ. Sci. Nano. 4 (2017) 1484-1493. doi:10.1039/C7EN00220C.

[81] Z. Hua, Z. Tang, X. Bai, J. Zhang, L. Yu, H. Cheng, Aggregation and resuspension of graphene oxide in simulated natural surface aquatic environments, Environ. Pollut. 205 (2015) 161-169. doi:https://doi.org/10.1016/j.envpol.2015.05.039.

[82] Y. Wang, P. Anilkumar, L. Cao, J.-H. Liu, P.G. Luo, K.N. Tackett, S. Sahu, P. Wang, X. Wang, Y.-P. Sun, Carbon dots of different composition and surface functionalization: cytotoxicity issues relevant to fluorescence cell imaging, Exp. Biol. Med. 236 (2011) 1231-1238. doi:10.1258/ebm.2011.011132.

[83] J. Shen, Y. Zhu, X. Yang, C. Li, Graphene quantum dots: emergent nanolights for bioimaging, sensors, catalysis and photovoltaic devices, Chem. Commun. 48 (2012) 3686-3699. doi:10.1039/C2CC00110A.

[84] Y. Wang, A. Hu, Carbon quantum dots: synthesis, properties and applications, J. Mater. Chem. C. 2 (2014) 6921-6939. doi:10.1039/C4TC00988F.

[85] P.-C. Hsu, Z.-Y. Shih, C.-H. Lee, H.-T. Chang, Synthesis and analytical applications of photoluminescent carbon nanodots, Green Chem. 14 (2012) 917920. doi:10.1039/C2GC16451E.

[86] X. Wang, K. Qu, B. Xu, J. Ren, X. Qu, Microwave assisted one-step green synthesis of cell-permeable multicolor photoluminescent carbon dots without surface passivation reagents, J. Mater. Chem. 21 (2011) 2445-2450. doi:10.1039/C0JM02963G.

[87] M. Troester, H.-J. Brauch, T. Hofmann, Vulnerability of drinking water supplies to engineered nanoparticles, Water Res. 96 (2016) 255-279. 
doi:https://doi.org/10.1016/j.watres.2016.03.038.

[88] X. Xu, R. Ray, Y. Gu, H.J. Ploehn, L. Gearheart, K. Raker, W.A. Scrivens, Electrophoretic Analysis and Purification of Fluorescent Single-Walled Carbon Nanotube Fragments, J. Am. Chem. Soc. 126 (2004) 12736-12737. doi:10.1021/ja040082h.

[89] Y.-P. Sun, B. Zhou, Y. Lin, W. Wang, K.A.S. Fernando, P. Pathak, M.J. Meziani, B.A. Harruff, X. Wang, H. Wang, P.G. Luo, H. Yang, M.E. Kose, B. Chen, L.M. Veca, S.-Y. Xie, Quantum-Sized Carbon Dots for Bright and Colorful Photoluminescence, J. Am. Chem. Soc. 128 (2006) 7756-7757. doi:10.1021/ja062677d.

[90] J. Zhou, C. Booker, R. Li, X. Zhou, T.-K. Sham, X. Sun, Z. Ding, An Electrochemical Avenue to Blue Luminescent Nanocrystals from Multiwalled Carbon Nanotubes (MWCNTs), J. Am. Chem. Soc. 129 (2007) 744-745. doi:10.1021/ja0669070.

[91] L. Zheng, Y. Chi, Y. Dong, J. Lin, B. Wang, Electrochemiluminescence of WaterSoluble Carbon Nanocrystals Released Electrochemically from Graphite, J. Am. Chem. Soc. 131 (2009) 4564-4565. doi:10.1021/ja809073f.

[92] Q.-L. Zhao, Z.-L. Zhang, B.-H. Huang, J. Peng, M. Zhang, D.-W. Pang, Facile preparation of low cytotoxicity fluorescent carbon nanocrystals by electrooxidation of graphite, Chem. Commun. (2008) 5116-5118. doi:10.1039/B812420E.

[93] H. Liu, T. Ye, C. Mao, Fluorescent Carbon Nanoparticles Derived from Candle Soot, Angew. Chemie Int. Ed. 46 (2007) 6473-6475. doi:10.1002/anie.200701271.

[94] L. Tian, D. Ghosh, W. Chen, S. Pradhan, X. Chang, S. Chen, Nanosized Carbon Particles From Natural Gas Soot, Chem. Mater. 21 (2009) 2803-2809. doi:10.1021/cm900709w.

[95] D.R. Dreyer, R.S. Ruoff, C.W. Bielawski, From Conception to Realization: An Historial Account of Graphene and Some Perspectives for Its Future, Angew. Chemie Int. Ed. 49 (2010) 9336-9344. doi:10.1002/anie.201003024.

[96] A.B. Bourlinos, A. Stassinopoulos, D. Anglos, R. Zboril, M. Karakassides, E.P. Giannelis, Surface Functionalized Carbogenic Quantum Dots, Small. 4 (2008) 455-458. doi:10.1002/smll.200700578.

[97] R. Liu, D. Wu, S. Liu, K. Koynov, W. Knoll, Q. Li, An Aqueous Route to Multicolor Photoluminescent Carbon Dots Using Silica Spheres as Carriers, Angew. Chemie Int. Ed. 48 (2009) 4598-4601. doi:10.1002/anie.200900652.

[98] H. Zhu, X. Wang, Y. Li, Z. Wang, F. Yang, X. Yang, Microwave synthesis of 
fluorescent carbon nanoparticles with electrochemiluminescence properties, Chem. Commun. (2009) 5118-5120. doi:10.1039/B907612C.

[99] Y. Xu, K. Sheng, C. Li, G. Shi, Self-Assembled Graphene Hydrogel via a OneStep Hydrothermal Process, ACS Nano. 4 (2010) 4324-4330.

doi:10.1021/nn101187z.

[100] R. Ye, Y. Liu, Z. Peng, T. Wang, A.S. Jalilov, B.I. Yakobson, S.-H. Wei, J.M. Tour, High Performance Electrocatalytic Reaction of Hydrogen and Oxygen on Ruthenium Nanoclusters, ACS Appl. Mater. Interfaces. 9 (2017) 3785-3791. doi:10.1021/acsami.6b15725.

[101] Z. Chen, W. Ren, L. Gao, B. Liu, S. Pei, H.-M. Cheng, Three-dimensional flexible and conductive interconnected graphene networks grown by chemical vapour deposition, Nat. Mater. 10 (2011) 424. http://dx.doi.org/10.1038/nmat3001.

[102] B.G. Choi, M. Yang, W.H. Hong, J.W. Choi, Y.S. Huh, 3D Macroporous Graphene Frameworks for Supercapacitors with High Energy and Power Densities, ACS Nano. 6 (2012) 4020-4028. doi:10.1021/nn3003345.

[103] R. Ye, D.K. James, J.M. Tour, Laser-Induced Graphene, Acc. Chem. Res. 51 (2018) 1609-1620. doi:10.1021/acs.accounts.8b00084.

[104] Y. Dong, S.C. Rismiller, J. Lin, Molecular dynamic simulation of layered graphene clusters formation from polyimides under extreme conditions, Carbon $\mathrm{N}$. Y. 104 (2016) 47-55. doi:https://doi.org/10.1016/j.carbon.2016.03.050.

[105] L.X. Duy, Z. Peng, Y. Li, J. Zhang, Y. Ji, J.M. Tour, Laser-induced graphene fibers, Carbon N. Y. 126 (2018) 472-479. doi:https://doi.org/10.1016/j.carbon.2017.10.036.

[106] L. Lai, J.R. Potts, D. Zhan, L. Wang, C.K. Poh, C. Tang, H. Gong, Z. Shen, J. Lin, R.S. Ruoff, Exploration of the active center structure of nitrogen-doped graphenebased catalysts for oxygen reduction reaction, Energy Environ. Sci. 5 (2012) 7936-7942. doi:10.1039/C2EE21802J.

[107] B. Qiu, M. Xing, J. Zhang, Mesoporous TiO2 Nanocrystals Grown in Situ on Graphene Aerogels for High Photocatalysis and Lithium-Ion Batteries, J. Am. Chem. Soc. 136 (2014) 5852-5855. doi:10.1021/ja500873u.

[108] M.P. Kumar, M.M. Raju, A. Arunchander, S. Selvaraj, G. Kalita, T.N. Narayanan, A.K. Sahu, D.K. Pattanayak, Nitrogen Doped Graphene as Metal Free Electrocatalyst for Efficient Oxygen Reduction Reaction in Alkaline Media and Its Application in Anion Exchange Membrane Fuel Cells, J. Electrochem. Soc. . 163 (2016) F848-F855. http://jes.ecsdl.org/content/163/8/F848.abstract. 
[109] Z. Peng, R. Ye, J.A. Mann, D. Zakhidov, Y. Li, P.R. Smalley, J. Lin, J.M. Tour, Flexible Boron-Doped Laser-Induced Graphene Microsupercapacitors, ACS Nano. 9 (2015) 5868-5875. doi:10.1021/acsnano.5b00436.

[110] Y. Li, D.X. Luong, J. Zhang, Y.R. Tarkunde, C. Kittrell, F. Sargunaraj, Y. Ji, C.J. Arnusch, J.M. Tour, Laser-Induced Graphene in Controlled Atmospheres: From Superhydrophilic to Superhydrophobic Surfaces, Adv. Mater. 29 (2017) 1700496. doi:10.1002/adma.201700496.

[111] P. Nayak, N. Kurra, C. Xia, H.N. Alshareef, Highly Efficient Laser Scribed Graphene Electrodes for On-Chip Electrochemical Sensing Applications, Adv. Electron. Mater. 2 (2016) 1600185. doi:10.1002/aelm.201600185.

[112] R. Ye, Y. Chyan, J. Zhang, Y. Li, X. Han, C. Kittrell, J.M. Tour, Laser-Induced Graphene Formation on Wood, Adv. Mater. 29 (2017) 1702211. doi:10.1002/adma.201702211.

[113] K.W. Tan, B. Jung, J.G. Werner, E.R. Rhoades, M.O. Thompson, U. Wiesner, Transient laser heating induced hierarchical porous structures from block copolymer-directed self-assembly, Science (80-. ). 349 (2015) 54 LP - 58. http://science.sciencemag.org/content/349/6243/54.abstract.

[114] L.-Q. Tao, H. Tian, Y. Liu, Z.-Y. Ju, Y. Pang, Y.-Q. Chen, D.-Y. Wang, X.-G. Tian, J.-C. Yan, N.-Q. Deng, Y. Yang, T.-L. Ren, An intelligent artificial throat with sound-sensing ability based on laser induced graphene, Nat. Commun. 8 (2017) 14579. http://dx.doi.org/10.1038/ncomms14579.

[115] R. Ye, Z. Peng, T. Wang, Y. Xu, J. Zhang, Y. Li, L.G. Nilewski, J. Lin, J.M. Tour, In Situ Formation of Metal Oxide Nanocrystals Embedded in Laser-Induced Graphene, ACS Nano. 9 (2015) 9244-9251. doi:10.1021/acsnano.5b04138.

[116] J. Zhang, C. Zhang, J. Sha, H. Fei, Y. Li, J.M. Tour, Efficient Water-Splitting Electrodes Based on Laser-Induced Graphene, ACS Appl. Mater. Interfaces. 9 (2017) 26840-26847. doi:10.1021/acsami.7b06727.

[117] C.M. Tittle, D. Yilman, M.A. Pope, C.J. Backhouse, Robust Superhydrophobic Laser-Induced Graphene for Desalination Applications, Adv. Mater. Technol. 3 (2017) 1700207. doi:10.1002/admt.201700207.

[118] A. Cayuela, M.L. Soriano, M. Valcárcel, Photoluminescent carbon dot sensor for carboxylated multiwalled carbon nanotube detection in river water, Sensors Actuators B Chem. 207 (2015) 596-601. doi:https://doi.org/10.1016/j.snb.2014.10.102.

[119] S. Zhu, Y. Song, X. Zhao, J. Shao, J. Zhang, B. Yang, The photoluminescence 
mechanism in carbon dots (graphene quantum dots, carbon nanodots, and polymer dots): current state and future perspective, Nano Res. 8 (2015) 355-381. doi:10.1007/s12274-014-0644-3.

[120] H. Li, X. He, Z. Kang, H. Huang, Y. Liu, J. Liu, S. Lian, C.H.A. Tsang, X. Yang, S.-T. Lee, Water-Soluble Fluorescent Carbon Quantum Dots and Photocatalyst Design, Angew. Chemie Int. Ed. 49 (2010) 4430-4434.

doi:10.1002/anie.200906154.

[121] Y. Fang, S. Guo, D. Li, C. Zhu, W. Ren, S. Dong, E. Wang, Easy Synthesis and Imaging Applications of Cross-Linked Green Fluorescent Hollow Carbon Nanoparticles, ACS Nano. 6 (2012) 400-409. doi:10.1021/nn2046373.

[122] S.-L. Hu, K.-Y. Niu, J. Sun, J. Yang, N.-Q. Zhao, X.-W. Du, One-step synthesis of fluorescent carbon nanoparticles by laser irradiation, J. Mater. Chem. 19 (2009) 484-488. doi:10.1039/B812943F.

[123] S. Srivastava, N.S. Gajbhiye, Carbogenic Nanodots: Photoluminescence and Room-Temperature Ferromagnetism, ChemPhysChem. 12 (2011) 2624-2632. doi:10.1002/cphc.201100188.

[124] J.C.G. Esteves da Silva, H.M.R. Gonçalves, Analytical and bioanalytical applications of carbon dots, TrAC Trends Anal. Chem. 30 (2011) 1327-1336. doi:https://doi.org/10.1016/j.trac.2011.04.009.

[125] H. Tao, K. Yang, Z. Ma, J. Wan, Y. Zhang, Z. Kang, Z. Liu, In vivo NIR fluorescence imaging, biodistribution, and toxicology of photoluminescent carbon dots produced from carbon nanotubes and graphite., Small. 8 (2012) 281-290. doi:10.1002/smll.201101706.

[126] Y. Liu, C. Liu, Z. Zhang, Synthesis of highly luminescent graphitized carbon dots and the application in the $\mathrm{Hg} 2+$ detection, Appl. Surf. Sci. 263 (2012) 481-485. doi:https://doi.org/10.1016/j.apsusc.2012.09.088.

[127] P. Vazquez-Roig, R. Segarra, C. Blasco, V. Andreu, Y. Picó, Determination of pharmaceuticals in soils and sediments by pressurized liquid extraction and liquid chromatography tandem mass spectrometry, J. Chromatogr. A. 1217 (2010) 24712483. doi:https://doi.org/10.1016/j.chroma.2009.11.033.

[128] R. Jelinek, Carbon Quantum Dots: Synthesis, Properties and Applications (Carbon Nanostructures), Springer International Publishing, Switzerland, 2017. https://link.springer.com/book/10.1007/978-3-319-43911-2\#toc.

[129] M. Zheng, S. Liu, J. Li, D. Qu, H. Zhao, X. Guan, X. Hu, Z. Xie, X. Jing, Z. Sun, Integrating Oxaliplatin with Highly Luminescent Carbon Dots: An Unprecedented 
Theranostic Agent for Personalized Medicine, Adv. Mater. 26 (2014) 3554-3560. doi:10.1002/adma.201306192.

[130] J. Gregory, Interaction of unequal double layers at constant charge, J. Colloid Interface Sci. 51 (1975) 44-51. doi:https://doi.org/10.1016/0021-9797(75)90081-8.

[131] J. Gregory, Approximate expressions for retarded van der waals interaction, J. Colloid Interface Sci. 83 (1981) 138-145. doi:https://doi.org/10.1016/00219797(81)90018-7.

[132] L. Feriancikova, S. Xu, Deposition and remobilization of graphene oxide within saturated sand packs, J. Hazard. Mater. 235-236 (2012) 194-200. doi:https://doi.org/10.1016/j.jhazmat.2012.07.041.

[133] J. Tang, B. Kong, H. Wu, M. Xu, Y. Wang, Y. Wang, D. Zhao, G. Zheng, Carbon Nanodots Featuring Efficient FRET for Real-Time Monitoring of Drug Delivery and Two-Photon Imaging, Adv. Mater. 25 (2013) 6569-6574. doi:10.1002/adma.201303124.

[134] E.J.W. Verwey, Theory of the Stability of Lyophobic Colloids., J. Phys. Colloid Chem. 51 (1947) 631-636. doi:10.1021/j150453a001.

[135] M. Elimelech, J. Gregory, X. Jia, R.A. Williams, Preface BT - Particle Deposition \& Aggregation, in: Butterworth-Heinemann, Woburn, 1995: pp. xiii-xv. doi:https://doi.org/10.1016/B978-075067024-1/50000-5.

[136] D. Dubin, Ion adventure in the heartland: exploring the heart's ion, Cover Publishing Company, 2003.

[137] G.R. Stark, Reactions of Cyanate with Functional Groups of Proteins. IV. Inertness of Aliphatic Hydroxyl Groups. Formation of Carbamyl- and Acylhydantoins*, Biochemistry. 4 (1965) 2363-2367. doi:10.1021/bi00887a015.

[138] S. Wagner, A. Gondikas, E. Neubauer, T. Hofmann, F. von der Kammer, Spot the Difference: Engineered and Natural Nanoparticles in the Environment-Release, Behavior, and Fate, Angew. Chemie Int. Ed. 53 (2014) 12398-12419. doi:10.1002/anie.201405050.

[139] X. Jia, J. Li, E. Wang, One-pot green synthesis of optically pH-sensitive carbon dots with upconversion luminescence, Nanoscale. 4 (2012) 5572-5575. doi:10.1039/C2NR31319G.

[140] W. Davison, C. Woof, Performance tests for the measurement of $\mathrm{pH}$ with glass electrodes in low ionic strength solutions including natural waters, Anal. Chem. 57 (1985) 2567-2570. doi:10.1021/ac00290a031. 
[141] S. Deng, G. Yu, Y.P. Ting, Removal of Humic Acid Using PEI-Modified Fungal Biomass, Sep. Sci. Technol. 41 (2006) 2989-3002. doi:10.1080/01496390600786028.

[142] Q. Tao, Z. Xu, J. Wang, F. Liu, H. Wan, S. Zheng, Adsorption of humic acid to aminopropyl functionalized SBA-15, Microporous Mesoporous Mater. 131 (2010) 177-185. doi:https://doi.org/10.1016/j.micromeso.2009.12.018.

[143] X. Zhang, R. Bai, Mechanisms and kinetics of humic acid adsorption onto chitosan-coated granules., J. Colloid Interface Sci. 264 (2003) 30-38. doi:10.1016/s0021-9797(03)00393-x.

[144] A.J. Pelley, N. Tufenkji, Effect of particle size and natural organic matter on the migration of nano- and microscale latex particles in saturated porous media, J. Colloid Interface Sci. 321 (2008) 74-83. doi:https://doi.org/10.1016/j.jcis.2008.01.046.

[145] F. Part, C. Zaba, O. Bixner, C. Zafiu, S. Hann, E.-K. Sinner, M. Huber-Humer, Traceability of fluorescent engineered nanomaterials and their fate in complex liquid waste matrices, Environ. Pollut. 214 (2016) 795-805. doi:https://doi.org/10.1016/j.envpol.2016.04.032.

[146] G. Ruan, Z. Sun, Z. Peng, J.M. Tour, Growth of Graphene from Food, Insects, and Waste, ACS Nano. 5 (2011) 7601-7607. doi:10.1021/nn202625c.

[147] O.C. Compton, S.T. Nguyen, Graphene Oxide, Highly Reduced Graphene Oxide, and Graphene: Versatile Building Blocks for Carbon-Based Materials, Small. 6 (2010) 711-723. doi:10.1002/smll.200901934.

[148] E.P. Randviir, D.A.C. Brownson, C.E. Banks, A decade of graphene research: production, applications and outlook, Mater. Today. 17 (2014) 426-432. doi:https://doi.org/10.1016/j.mattod.2014.06.001.

[149] K.S. Novoselov, V.I. Fal[prime]ko, L. Colombo, P.R. Gellert, M.G. Schwab, K. Kim, A roadmap for graphene, Nature. 490 (2012) 192-200. http://dx.doi.org/10.1038/nature11458.

[150] W. Gao, M. Majumder, L.B. Alemany, T.N. Narayanan, M.A. Ibarra, B.K. Pradhan, P.M. Ajayan, Engineered Graphite Oxide Materials for Application in Water Purification, ACS Appl. Mater. Interfaces. 3 (2011) 1821-1826. doi:10.1021/am200300u.

[151] D. Cohen-Tanugi, J.C. Grossman, Water Desalination across Nanoporous Graphene, Nano Lett. 12 (2012) 3602-3608. doi:10.1021/nl3012853.

[152] Review of Potential Environmental and Health Risks of the Nanomaterial 
Graphene, Hum. Ecol. Risk Assess. An Int. J. 19 (2013) 873-887.

doi:10.1080/10807039.2012.702039.

[153] A.M. Jastrzębska, A.R. Olszyna, The ecotoxicity of graphene family materials: current status, knowledge gaps and future needs, J. Nanoparticle Res. 17 (2015) 40. doi:10.1007/s11051-014-2817-0.

[154] L. Chen, P. Hu, L. Zhang, S. Huang, L. Luo, C. Huang, Toxicity of graphene oxide and multi-walled carbon nanotubes against human cells and zebrafish, Sci. China Chem. 55 (2012) 2209-2216. doi:10.1007/s11426-012-4620-z.

[155] O. Akhavan, E. Ghaderi, A. Esfandiar, Wrapping Bacteria by Graphene Nanosheets for Isolation from Environment, Reactivation by Sonication, and Inactivation by Near-Infrared Irradiation, J. Phys. Chem. B. 115 (2011) 62796288. doi:10.1021/jp200686k.

[156] O. Suárez-Iglesias, S. Collado, P. Oulego, M. Díaz, Graphene-family nanomaterials in wastewater treatment plants, Chem. Eng. J. 313 (2017) 121-135. doi:https://doi.org/10.1016/j.cej.2016.12.022.

[157] K. Yang, B. Chen, X. Zhu, B. Xing, Aggregation, Adsorption, and Morphological Transformation of Graphene Oxide in Aqueous Solutions Containing Different Metal Cations, Environ. Sci. Technol. 50 (2016) 11066-11075. doi:10.1021/acs.est.6b04235.

[158] Y. Jiang, R. Raliya, J.D. Fortner, P. Biswas, Graphene Oxides in Water: Correlating Morphology and Surface Chemistry with Aggregation Behavior, Environ. Sci. Technol. 50 (2016) 6964-6973. doi:10.1021/acs.est.6b00810.

[159] M.M. Gudarzi, Colloidal Stability of Graphene Oxide: Aggregation in Two Dimensions, Langmuir. 32 (2016) 5058-5068. doi:10.1021/acs.langmuir.6b01012.

[160] D.G. Papageorgiou, I.A. Kinloch, R.J. Young, Graphene/elastomer nanocomposites, Carbon N. Y. 95 (2015) 460-484. doi:https://doi.org/10.1016/j.carbon.2015.08.055.

[161] J. Zhao, F. Liu, Z. Wang, X. Cao, B. Xing, Heteroaggregation of Graphene Oxide with Minerals in Aqueous Phase, Environ. Sci. Technol. 49 (2015) 2849-2857. doi:10.1021/es505605w.

[162] D. Li, M.B. Muller, S. Gilje, R.B. Kaner, G.G. Wallace, Processable aqueous dispersions of graphene nanosheets, Nat Nano. 3 (2008) 101-105. http://dx.doi.org/10.1038/nnano.2007.451.

[163] S. Park, K.-S. Lee, G. Bozoklu, W. Cai, S.T. Nguyen, R.S. Ruoff, Graphene Oxide Papers Modified by Divalent Ions-Enhancing Mechanical Properties via 
Chemical Cross-Linking, ACS Nano. 2 (2008) 572-578. doi:10.1021/nn700349a.

[164] M.M. Emara, N.A. Farid, A.M. Wasfi, M.M. Bahr, H.M. Abd-Elbary, Thermodynamics of ion association in aqueous solutions of calcium- and magnesium-substituted hydroxybenzoates using an ion-selective electrode technique, J. Phys. Chem. 88 (1984) 3345-3348. doi:10.1021/j150659a042.

[165] A. Ouatmane, M. Hafidi, M. EL Gharous, J. Revel C., Complexation of calcium ions by humic and fulvic acids, Analusis. 27 (1999) 428-431. https://doi.org/10.1051/analusis:1999270428.

[166] J.C. Crittenden, M.W.H. (Firm), Water treatment principles and design, J. Wiley, Hoboken, N.J., 2005.

[167] F.H. Frimmel, Chemistry of the Solid-Water Interface. Processes at the MineralWater and Particle-Water Interface in Natural Systems. Von W. Stumm. Wiley, Chichester, 1992. X, 428 S., Broschur 32.50£ - ISBN 0-471-57672-7, Angew. Chemie. 105 (1993) 800. doi:10.1002/ange.19931050543.

[168] G. Sposito 1939-, The surface chemistry of soils / Garrison Sposito, Oxford University Press ; Clarendon Press, New York : Oxford [Oxfordshire], 1984.

[169] B.S. Archanjo, J.R. Araujo, A.M. Silva, R.B. Capaz, N.P.S. Falcão, A. Jorio, C.A. Achete, Chemical Analysis and Molecular Models for Calcium-Oxygen-Carbon Interactions in Black Carbon Found in Fertile Amazonian Anthrosoils, Environ. Sci. Technol. 48 (2014) 7445-7452. doi:10.1021/es501046b.

[170] K.H. Chu, Y. Huang, M. Yu, N. Her, J.R. V Flora, C.M. Park, S. Kim, J. Cho, Y. Yoon, Evaluation of Humic Acid and Tannic Acid Fouling in Graphene OxideCoated Ultrafiltration Membranes, ACS Appl. Mater. Interfaces. 8 (2016) 2227022279. doi:10.1021/acsami.6b08020.

[171] D. Lin, B. Xing, Tannic Acid Adsorption and Its Role for Stabilizing Carbon Nanotube Suspensions, Environ. Sci. Technol. 42 (2008) 5917-5923. doi:10.1021/es800329c.

[172] S.P. Surwade, S.N. Smirnov, I. V Vlassiouk, R.R. Unocic, G.M. Veith, S. Dai, S.M. Mahurin, Water desalination using nanoporous single-layer graphene, Nat. Nanotechnol. 10 (2015) 459. http://dx.doi.org/10.1038/nnano.2015.37.

[173] D. M., J. N., N. B., Designing Robust Preparative Purification Processes with High Performance, Chem. Eng. Technol. 31 (2008) 875-882. doi:10.1002/ceat.200800097.

[174] M. Elimelech, W.A. Phillip, The Future of Seawater Desalination: Energy, Technology, and the Environment, Science (80-. ). 333 (2011) 712 LP - 717. 
http://science.sciencemag.org/content/333/6043/712.abstract.

[175] D.L. Gin, R.D. Noble, Designing the Next Generation of Chemical Separation Membranes, Science (80-. ). 332 (2011) 674 LP - 676. http://science.sciencemag.org/content/332/6030/674.abstract.

[176] J. Zhang, Z. Xu, M. Shan, B. Zhou, Y. Li, B. Li, J. Niu, X. Qian, Synergetic effects of oxidized carbon nanotubes and graphene oxide on fouling control and anti-fouling mechanism of polyvinylidene fluoride ultrafiltration membranes, J. Memb. Sci. 448 (2013) 81-92. doi:https://doi.org/10.1016/j.memsci.2013.07.064.

[177] J. Lee, H.-R. Chae, Y.J. Won, K. Lee, C.-H. Lee, H.H. Lee, I.-C. Kim, J. Lee, Graphene oxide nanoplatelets composite membrane with hydrophilic and antifouling properties for wastewater treatment, J. Memb. Sci. 448 (2013) $223-$ 230. doi:https://doi.org/10.1016/j.memsci.2013.08.017.

[178] L. Yu, Y. Zhang, B. Zhang, J. Liu, H. Zhang, C. Song, Preparation and characterization of HPEI-GO/PES ultrafiltration membrane with antifouling and antibacterial properties, J. Memb. Sci. 447 (2013) 452-462. doi:https://doi.org/10.1016/j.memsci.2013.07.042.

[179] H. Huang, Y. Ying, X. Peng, Graphene oxide nanosheet: an emerging star material for novel separation membranes, J. Mater. Chem. A. 2 (2014) 13772-13782. doi:10.1039/C4TA02359E.

[180] L. Tsetseris, S.T. Pantelides, Graphene: An impermeable or selectively permeable membrane for atomic species?, Carbon N. Y. 67 (2014) 58-63. doi:https://doi.org/10.1016/j.carbon.2013.09.055.

[181] J.-G. Gai, X.-L. Gong, W.-W. Wang, X. Zhang, W.-L. Kang, An ultrafast water transport forward osmosis membrane: porous graphene, J. Mater. Chem. A. 2 (2014) 4023-4028. doi:10.1039/C3TA14256F.

[182] H. Liu, S. Dai, D. Jiang, Permeance of $\mathrm{H} 2$ through porous graphene from molecular dynamics, Solid State Commun. 175-176 (2013) 101-105. doi:https://doi.org/10.1016/j.ssc.2013.07.004.

[183] A.K. Geim, Graphene: Status and Prospects, Science (80-. ). 324 (2009) 1530 LP 1534. http://science.sciencemag.org/content/324/5934/1530.abstract.

[184] H. Yu, B. Zhang, C. Bulin, R. Li, R. Xing, High-efficient Synthesis of Graphene Oxide Based on Improved Hummers Method, Sci. Rep. 6 (2016) 36143. https://doi.org/10.1038/srep36143.

[185] L. Li, J. Zhang, Z. Peng, Y. Li, C. Gao, Y. Ji, R. Ye, N.D. Kim, Q. Zhong, Y. Yang, H. Fei, G. Ruan, J.M. Tour, High-Performance Pseudocapacitive 
Microsupercapacitors from Laser-Induced Graphene, Adv. Mater. 28 (2015) 838845. doi:10.1002/adma.201503333.

[186] D.R. Machado, D. Hasson, R. Semiat, Effect of solvent properties on permeate flow through nanofiltration membranes. Part I: investigation of parameters affecting solvent flux, J. Memb. Sci. 163 (1999) 93-102. doi:https://doi.org/10.1016/S0376-7388(99)00158-1.

[187] A. Dobrak, B. Verrecht, H. Van den Dungen, A. Buekenhoudt, I.F.J. Vankelecom, B. Van der Bruggen, Solvent flux behavior and rejection characteristics of hydrophilic and hydrophobic mesoporous and microporous $\mathrm{TiO} 2$ and $\mathrm{ZrO} 2$ membranes, J. Memb. Sci. 346 (2010) 344-352. doi:https://doi.org/10.1016/j.memsci.2009.09.059.

[188] C.R. Tanardi, I.F.J. Vankelecom, A.F.M. Pinheiro, K.K.R. Tetala, A. Nijmeijer, L. Winnubst, Solvent permeation behavior of PDMS grafted $\gamma$-alumina membranes, J. Memb. Sci. 495 (2015) 216-225. doi:https://doi.org/10.1016/j.memsci.2015.08.004.

[189] A. Buekenhoudt, F. Bisignano, G. De Luca, P. Vandezande, M. Wouters, K. Verhulst, Unravelling the solvent flux behaviour of ceramic nanofiltration and ultrafiltration membranes, J. Memb. Sci. 439 (2013) 36-47. doi:https://doi.org/10.1016/j.memsci.2013.03.032.

[190] C. Andecochea Saiz, S. Darvishmanesh, A. Buekenhoudt, B. Van der Bruggen, Shortcut applications of the Hansen Solubility Parameter for Organic Solvent Nanofiltration, J. Memb. Sci. 546 (2018) 120-127. doi:https://doi.org/10.1016/j.memsci.2017.10.016.

[191] W. Stöber, A. Fink, E. Bohn, Controlled growth of monodisperse silica spheres in the micron size range, J. Colloid Interface Sci. 26 (1968) 62-69. doi:https://doi.org/10.1016/0021-9797(68)90272-5.

[192] H. Xie, K.L. Gill-Sharp, D.P. O’Neal, Quantitative estimation of gold nanoshell concentrations in whole blood using dynamic light scattering, Nanomedicine Nanotechnology, Biol. Med. 3 (2007) 89-94. doi:https://doi.org/10.1016/j.nano.2007.01.003.

[193] I. Makra, P. Terejánszky, R.E. Gyurcsányi, A method based on light scattering to estimate the concentration of virus particles without the need for virus particle standards, MethodsX. 2 (2015) 91-99. doi:https://doi.org/10.1016/j.mex.2015.02.003.

[194] M. Kotera, T. Nishino, K. Nakamae, Imidization processes of aromatic polyimide by temperature modulated DSC, Polymer (Guildf). 41 (2000) 3615-3619. 
doi:https://doi.org/10.1016/S0032-3861(99)00546-7.

[195] F.C. Tai, S.C. Lee, C.H. Wei, S.L. Tyan, Correlation between ID/IG Ratio from Visible Raman Spectra and sp2/sp3 Ratio from XPS Spectra of Annealed Hydrogenated DLC, Mater. Trans. 47 (2006) 1847-1852. doi:10.2320/matertrans.47.1847.

[196] S. Diaham, M.L. Locatelli, T. Lebey, D. Malec, Thermal imidization optimization of polyimide thin films using Fourier transform infrared spectroscopy and electrical measurements, Thin Solid Films. 519 (2011) 1851-1856. doi:https://doi.org/10.1016/j.tsf.2010.10.031.

[197] A.C. Ferrari, J.C. Meyer, V. Scardaci, C. Casiraghi, M. Lazzeri, F. Mauri, S. Piscanec, D. Jiang, K.S. Novoselov, S. Roth, A.K. Geim, Raman Spectrum of Graphene and Graphene Layers, Phys. Rev. Lett. 97 (2006) 187401. https://link.aps.org/doi/10.1103/PhysRevLett.97.187401.

[198] Physisorption of gases, with special reference to the evaluation of surface area and pore size distribution (IUPAC Technical Report) , Pure Appl. Chem. . 87 (2015) 1051. doi:10.1515/pac-2014-1117.

[199] M. Bayati, H. Peng, H. Deng, J. Lin, M. Fidalgo, Graphene Coated Microfiltration Ceramic Membrane Fabricated by Photothermic Conversion of Polyimide, MRS Adv. 2 (2017) 2489-2495. doi:10.1557/adv.2017.398.

[200] E. Bonaccurso, M. Kappl, H.-J. Butt, Hydrodynamic Force Measurements: Boundary Slip of Water on Hydrophilic Surfaces and Electrokinetic Effects, Phys. Rev. Lett. 88 (2002) 76103. https://link.aps.org/doi/10.1103/PhysRevLett.88.076103.

[201] Q. Yang, Y. Su, C. Chi, C.T. Cherian, K. Huang, V.G. Kravets, F.C. Wang, J.C. Zhang, A. Pratt, A.N. Grigorenko, F. Guinea, A.K. Geim, R.R. Nair, Ultrathin graphene-based membrane with precise molecular sieving and ultrafast solvent permeation, Nat. Mater. 16 (2017) 1198. http://dx.doi.org/10.1038/nmat5025.

[202] H. Liang, C. Ji, G. Tiantian, Z. Miao, L. Yingru, D. Liming, Q. Liangti, S. Gaoquan, Reduced Graphene Oxide Membranes for Ultrafast Organic Solvent Nanofiltration, Adv. Mater. 28 (2016) 8669-8674. doi:10.1002/adma.201601606.

[203] X.J. Yang, A.G. Livingston, L. Freitas dos Santos, Experimental observations of nanofiltration with organic solvents, J. Memb. Sci. 190 (2001) 45-55. doi:https://doi.org/10.1016/S0376-7388(01)00392-1.

[204] S. Zeidler, U. Kätzel, P. Kreis, Systematic investigation on the influence of solutes 
on the separation behavior of a PDMS membrane in organic solvent nanofiltration, J. Memb. Sci. 429 (2013) 295-303.

doi:https://doi.org/10.1016/j.memsci.2012.11.056.

[205] S. Darvishmanesh, B. Van der Bruggen, Mass Transport through Nanostructured Membranes: Towards a Predictive Tool, Membr. . 6 (2016).

doi:10.3390/membranes6040049.

[206] T. Tsuru, M. Miyawaki, H. Kondo, T. Yoshioka, M. Asaeda, Inorganic porous membranes for nanofiltration of nonaqueous solutions, Sep. Purif. Technol. 32 (2003) 105-109. doi:https://doi.org/10.1016/S1383-5866(03)00074-1.

[207] S.J. Kim, D.W. Kim, K.M. Cho, K.M. Kang, J. Choi, D. Kim, H.-T. Jung, Ultrathin graphene oxide membranes on freestanding carbon nanotube supports for enhanced selective permeation in organic solvents, Sci. Rep. 8 (2018) 1959. doi:10.1038/s41598-018-19795-z.

[208] C.S. Castro, M.C. Guerreiro, M. Gonçalves, L.C.A. Oliveira, A.S. Anastácio, Activated carbon/iron oxide composites for the removal of atrazine from aqueous medium, J. Hazard. Mater. 164 (2009) 609-614. doi:https://doi.org/10.1016/j.jhazmat.2008.08.066.

[209] K.S. Almberg, M.E. Turyk, R.M. Jones, K. Rankin, S. Freels, L.T. Stayner, Atrazine Contamination of Drinking Water and Adverse Birth Outcomes in Community Water Systems with Elevated Atrazine in Ohio, 2006 2008, Int. J. Environ. Res. Public Health. 15 (2018) 1889. doi:10.3390/ijerph15091889.

[210] W.E. Pereira, C.E. Rostad, Occurrence, distributions, and transport of herbicides and their degradation products in the Lower Mississippi River and its tributaries, Environ. Sci. Technol. 24 (1990) 1400-1406. doi:10.1021/es00079a015.

[211] M.S. Christin, L. Ménard, A.D. Gendron, S. Ruby, D. Cyr, D.J. Marcogliese, L. Rollins-Smith, M. Fournier, Effects of agricultural pesticides on the immune system of Xenopus laevis and Rana pipiens, Aquat. Toxicol. 67 (2004) 33-43. doi:https://doi.org/10.1016/j.aquatox.2003.11.007.

[212] D. Bennett, W.E. Kastenberg, T.E. McKone, A multimedia, multiple pathway risk assessment of atrazine: the impact of age differentiated exposure including joint uncertainty and variability, Reliab. Eng. Syst. Saf. 63 (1999) 185-198. doi:https://doi.org/10.1016/S0951-8320(98)00046-5.

[213] J. Bethsass, A. Colangelo, European Union Bans Atrazine, While the United States Negotiates Continued Use, Int. J. Occup. Environ. Health. 12 (2006) 260-267. doi:10.1179/oeh.2006.12.3.260. 
[214] D.A. Laird, P.Y. Yen, W.C. Koskinen, T.R. Steinheimer, R.H. Dowdy, Sorption of atrazine on Soil Clay Components, Environ. Sci. Technol. 28 (1994) 1054-1061. doi:10.1021/es00055a014.

[215] P. Chingombe, B. Saha, R.J. Wakeman, Sorption of atrazine on conventional and surface modified activated carbons, J. Colloid Interface Sci. 302 (2006) 408-416. doi:https://doi.org/10.1016/j.jcis.2006.06.065.

[216] D.S. Gamble, Atrazine Sorption Kinetics In A Characterized Soil: Predictive Calculations, Environ. Sci. Technol. 42 (2008) 1537-1541. doi:10.1021/es702432v.

[217] G.-C. Chen, X.-Q. Shan, Y.-Q. Zhou, X. Shen, H.-L. Huang, S.U. Khan, Adsorption kinetics, isotherms and thermodynamics of atrazine on surface oxidized multiwalled carbon nanotubes, J. Hazard. Mater. 169 (2009) 912-918. doi:https://doi.org/10.1016/j.jhazmat.2009.04.034.

[218] I. Lupul, J. Yperman, R. Carleer, G. Gryglewicz, Adsorption of atrazine on hemp stem-based activated carbons with different surface chemistry, Adsorption. 21 (2015) 489-498. doi:10.1007/s10450-015-9689-1.

[219] M. Shirmardi, N. Alavi, E.C. Lima, A. Takdastan, A.H. Mahvi, A.A. Babaei, Removal of atrazine as an organic micro-pollutant from aqueous solutions: a comparative study, Process Saf. Environ. Prot. 103 (2016) 23-35. doi:https://doi.org/10.1016/j.psep.2016.06.014.

[220] L. Yue, C. Ge, D. Feng, H. Yu, H. Deng, B. Fu, Adsorption-desorption behavior of atrazine on agricultural soils in China, J. Environ. Sci. 57 (2017) 180-189. doi:https://doi.org/10.1016/j.jes.2016.11.002.

[221] A.S. Jalilov, Y. Li, J. Tian, J.M. Tour, Ultra-High Surface Area Activated Porous Asphalt for $\mathrm{CO} 2$ Capture through Competitive Adsorption at High Pressures, Adv. Energy Mater. 7 (2016) 1600693. doi:10.1002/aenm.201600693.

[222] R. V Salvatierra, A.-R.O. Raji, S.-K. Lee, Y. Ji, L. Li, J.M. Tour, Silicon Nanowires and Lithium Cobalt Oxide Nanowires in Graphene Nanoribbon Papers for Full Lithium Ion Battery, Adv. Energy Mater. 6 (2016) 1600918. doi:10.1002/aenm.201600918.

[223] Y. Shen, B. Chen, Sulfonated Graphene Nanosheets as a Superb Adsorbent for Various Environmental Pollutants in Water, Environ. Sci. Technol. 49 (2015) 7364-7372. doi:10.1021/acs.est.5b01057.

[224] H. Yan, H. Yang, A. Li, R. Cheng, pH-tunable surface charge of chitosan/graphene oxide composite adsorbent for efficient removal of multiple pollutants from water, 
Chem. Eng. J. 284 (2016) 1397-1405.

doi:https://doi.org/10.1016/j.cej.2015.06.030.

[225] J. Xiao, W. Lv, Z. Xie, Y. Tan, Y. Song, Q. Zheng, Environmentally friendly reduced graphene oxide as a broad-spectrum adsorbent for anionic and cationic dyes via $\pi-\pi$ interactions, J. Mater. Chem. A. 4 (2016) 12126-12135. doi:10.1039/C6TA04119A.

[226] O.G. Apul, Q. Wang, Y. Zhou, T. Karanfil, Adsorption of aromatic organic contaminants by graphene nanosheets: Comparison with carbon nanotubes and activated carbon, Water Res. 47 (2013) 1648-1654. doi:https://doi.org/10.1016/j.watres.2012.12.031.

[227] C. Thamaraiselvan, J. Wang, D.K. James, P. Narkhede, S.P. Singh, D. Jassby, J.M. Tour, C.J. Arnusch, Laser-induced graphene and carbon nanotubes as conductive carbon-based materials in environmental technology, Mater. Today. 34 (2020) 115-131. doi:https://doi.org/10.1016/j.mattod.2019.08.014.

[228] R. Ye, D.K. James, J.M. Tour, Laser-Induced Graphene: From Discovery to Translation, Adv. Mater. 31 (2019) 1803621. doi:10.1002/adma.201803621.

[229] R. Wang, A. N Meredith, M. Lee Jr, D. Deutsch, L. Miadzvedskaya, E. Braun, P. Pantano, S. Harper, R. Draper, Toxicity assessment and bioaccumulation in zebrafish embryos exposed to carbon nanotubes suspended in Pluronic ${ }^{\circledR}$ F-108, Nanotoxicology. 10 (2016) 689-698. doi:10.3109/17435390.2015.1107147.

[230] R. Wang, C. Mikoryak, S. Li, D. 2nd Bushdiecker, I.H. Musselman, P. Pantano, R.K. Draper, Cytotoxicity screening of single-walled carbon nanotubes: detection and removal of cytotoxic contaminants from carboxylated carbon nanotubes., Mol. Pharm. 8 (2011) 1351-1361. doi:10.1021/mp2001439.

[231] N. Kobayashi, H. Izumi, Y. Morimoto, Review of toxicity studies of carbon nanotubes, J. Occup. Health. 59 (2017) 394-407. doi:10.1539/joh.17-0089-RA.

[232] M. d'Amora, A. Lamberti, M. Fontana, S. Giordani, Toxicity assessment of laserinduced graphene by zebrafish during development, J. Phys. Mater. 3 (2020) 34008. doi:10.1088/2515-7639/ab9522.

[233] M. Bayati, H. Peng, H. Deng, J. Lin, M. Fidalgo de Cortalezzi, Laser induced graphene /ceramic membrane composite: Preparation and characterization, J. Memb. Sci. (2019) 117537. doi:https://doi.org/10.1016/j.memsci.2019.117537.

[234] M. Elimelech, J. Gregory, X. Jia, R.A. Williams, Chapter 2 - Electrical properties of interfaces, in: M. Elimelech, J. Gregory, X. Jia, R.A.B.T.-P.D.\& A. Williams (Eds.), Butterworth-Heinemann, Woburn, 1995: pp. 9-32. 
doi:https://doi.org/10.1016/B978-075067024-1/50002-9.

[235] C.D. Willett, R.N. Lerch, C.-H. Lin, K.W. Goyne, N.D. Leigh, C.A. Roberts, Identification of an atrazine-degrading benzoxazinoid in Eastern gamagrass (Tripsacum dactyloides)., J. Agric. Food Chem. 61 (2013) 8026-8033. doi:10.1021/jf402271h.

[236] W.J. Weber, J.C. Morris, Kinetics of adsorption carbon from solutions, J. Sanit. Engeering Div. Proceedings.American Soc. Civ. Eng. 89 (1963) 31-60.

[237] D.A. Almasri, N.B. Saleh, M.A. Atieh, G. McKay, S. Ahzi, Adsorption of phosphate on iron oxide doped halloysite nanotubes, Sci. Rep. 9 (2019) 3232. doi:10.1038/s41598-019-39035-2.

[238] C. Yang, Statistical Mechanical Study on the Freundlich Isotherm Equation, J. Colloid Interface Sci. 208 (1998) 379-387. doi:https://doi.org/10.1006/jcis.1998.5843.

[239] A. Dąbrowski, Adsorption - from theory to practice, Adv. Colloid Interface Sci. 93 (2001) 135-224. doi:https://doi.org/10.1016/S0001-8686(00)00082-8.

[240] H. Qiu, L. Lv, B. Pan, Q. Zhang, W. Zhang, Q. Zhang, Critical review in adsorption kinetic models, J. Zhejiang Univ. A. 10 (2009) 716-724. doi:10.1631/jzus.A0820524.

[241] H.K. Boparai, M. Joseph, D.M. O'Carroll, Kinetics and thermodynamics of cadmium ion removal by adsorption onto nano zerovalent iron particles, J. Hazard. Mater. 186 (2011) 458-465. doi:https://doi.org/10.1016/j.jhazmat.2010.11.029.

[242] N.S. and S.B. Singh, Removal of Atrazine, Metribuzin, Metolachlor and Alachlor by Granular Carbon, J. Environ. Anal. Toxicol. 3 (2013) 1-5. doi:10.4172/21610525.1000196.

[243] Q.-H. Tao, H.-X. Tang, Effect of dye compounds on the adsorption of atrazine by natural sediment, Chemosphere. 56 (2004) 31-38. doi:https://doi.org/10.1016/j.chemosphere.2004.02.029.

[244] G.-C. Chen, X.-Q. Shan, Y.-S. Wang, Z.-G. Pei, X.-E. Shen, B. Wen, G. Owens, Effects of Copper, Lead, and Cadmium on the Sorption and Desorption of Atrazine onto and from Carbon Nanotubes, Environ. Sci. Technol. 42 (2008) 8297-8302. doi:10.1021/es801376w.

[245] M.D. Ureña-Amate, M. Socías-Viciana, E. González-Pradas, M. Saifi, Effects of ionic strength and temperature on adsorption of atrazine by a heat treated kerolite, Chemosphere. 59 (2005) 69-74. doi:https://doi.org/10.1016/j.chemosphere.2004.09.098. 
[246] K.B. HURLE, V.H. FREED, EFFECT OF ELECTROLYTES ON THE SOLUBILITY OF SOME 1,3,5-TRIAZINES AND SUBSTITUTED UREAS AND THEIR ADSORPTION ON SOIL*, Weed Res. 12 (1972) 1-10. doi:10.1111/j.1365-3180.1972.tb01181.x.

[247] W.-W. Tang, G.-M. Zeng, J.-L. Gong, Y. Liu, X.-Y. Wang, Y.-Y. Liu, Z.-F. Liu, L. Chen, X.-R. Zhang, D.-Z. Tu, Simultaneous adsorption of atrazine and $\mathrm{Cu}$ (II) from wastewater by magnetic multi-walled carbon nanotube, Chem. Eng. J. 211212 (2012) 470-478. doi:https://doi.org/10.1016/j.cej.2012.09.102.

[248] N.A. Kulikova, I. V Perminova, Binding of Atrazine to Humic Substances from Soil, Peat, and Coal Related to Their Structure, Environ. Sci. Technol. 36 (2002) 3720-3724. doi:10.1021/es015778e.

[249] S. Salvestrini, P. Sagliano, P. Iovino, S. Capasso, C. Colella, Atrazine adsorption by acid-activated zeolite-rich tuffs, Appl. Clay Sci. 49 (2010) 330-335. doi:https://doi.org/10.1016/j.clay.2010.04.008.

[250] I.D. Kovaios, C.A. Paraskeva, P.G. Koutsoukos, A.C. Payatakes, Adsorption of atrazine on soils: Model study, J. Colloid Interface Sci. 299 (2006) 88-94. doi:https://doi.org/10.1016/j.jcis.2006.01.057.

[251] X. Zhao, W. Ouyang, F. Hao, C. Lin, F. Wang, S. Han, X. Geng, Properties comparison of biochars from corn straw with different pretreatment and sorption behaviour of atrazine, Bioresour. Technol. 147 (2013) 338-344. doi:https://doi.org/10.1016/j.biortech.2013.08.042.

[252] G.J. Welhouse, W.F. Bleam, Atrazine hydrogen-bonding potentials, Environ. Sci. Technol. 27 (1993) 494-500. doi:10.1021/es00040a007.

[253] I. Ali, Z. ALOthman, A. Al-Warthan, Sorption, kinetics and thermodynamics studies of atrazine herbicide removal from water using iron nano-composite material., Int. J. Environ. Sci. Technol. 13 (2016) 733-742. http://10.0.3.239/s13762-015-0919-6.

[254] X.M. Yan, B.Y. Shi, J.J. Lu, C.H. Feng, D.S. Wang, H.X. Tang, Adsorption and desorption of atrazine on carbon nanotubes, J. Colloid Interface Sci. 321 (2008) 30-38. doi:https://doi.org/10.1016/j.jcis.2008.01.047.

[255] A. Chaparadza, J.M. Hossenlopp, Adsorption kinetics, isotherms and thermodynamics of atrazine removal using a banana peel based sorbent, Water Sci. Technol. 65 (2012) 940-947. http://dx.doi.org/10.2166/wst.2012.935.

[256] N. Singh, Adsorption of herbicides on coal fly ash from aqueous solutions, J. Hazard. Mater. 168 (2009) 233-237. 
doi:https://doi.org/10.1016/j.jhazmat.2009.02.016.

[257] W. Zheng, M. Guo, T. Chow, D.N. Bennett, N. Rajagopalan, Sorption properties of greenwaste biochar for two triazine pesticides, J. Hazard. Mater. 181 (2010) 121-126. doi:https://doi.org/10.1016/j.jhazmat.2010.04.103.

[258] M.B. Jahir Bin Alam, Anil Kumar Dikshit, Efficacy of Adsorbents of 2,4-D and Atrazine removal from water environment, Glob. NEST J. 2 (2000) 139-148.

[259] A. Saha, D. Bhaduri, A. Pipariya, R. Kumar Ghosh, Linear and nonlinear sorption modelling for adsorption of atrazine onto activated peanut husk, Environ. Prog. Sustain. Energy. 36 (2017) 348-358. doi:10.1002/ep.12434.

[260] P.K. Boruah, B. Sharma, N. Hussain, M.R. Das, Magnetically recoverable $\mathrm{Fe} 3 \mathrm{O} 4 /$ graphene nanocomposite towards efficient removal of triazine pesticides from aqueous solution: Investigation of the adsorption phenomenon and specific ion effect, Chemosphere. 168 (2017) 1058-1067. doi:https://doi.org/10.1016/j.chemosphere.2016.10.103.

[261] R. Xing, J. He, P. Hao, W. Zhou, Graphene oxide-supported nanoscale zero-valent iron composites for the removal of atrazine from aqueous solution, Colloids Surfaces A Physicochem. Eng. Asp. 589 (2020) 124466. doi:https://doi.org/10.1016/j.colsurfa.2020.124466.

[262] M. Engel, B. Chefetz, Removal of triazine-based pollutants from water by carbon nanotubes: Impact of dissolved organic matter (DOM) and solution chemistry, Water Res. 106 (2016) 146-154. doi:https://doi.org/10.1016/j.watres.2016.09.051.

[263] L. Martin-Neto, E.M. Vieira, G. Sposito, Mechanism of Atrazine Sorption by Humic Acid: A Spectroscopic Study, Environ. Sci. Technol. 28 (1994) 1867-1873. doi:10.1021/es00060a017.

[264] C. Pelekani, V.L. Snoeyink, Competitive adsorption in natural water: role of activated carbon pore size, Water Res. 33 (1999) 1209-1219. doi:https://doi.org/10.1016/S0043-1354(98)00329-7.

[265] G. Newcombe, M. Drikas, R. Hayes, Influence of characterised natural organic material on activated carbon adsorption: II. Effect on pore volume distribution and adsorption of 2-methylisoborneol, Water Res. 31 (1997) 1065-1073. doi:https://doi.org/10.1016/S0043-1354(96)00325-9.

[266] S. Zhang, T. Shao, T. Karanfil, The effects of dissolved natural organic matter on the adsorption of synthetic organic chemicals by activated carbons and carbon nanotubes, Water Res. 45 (2011) 1378-1386. doi:https://doi.org/10.1016/j.watres.2010.10.023. 
[267] X. Zhang, M. Kah, M.T.O. Jonker, T. Hofmann, Dispersion State and Humic Acids Concentration-Dependent Sorption of Pyrene to Carbon Nanotubes, Environ. Sci. Technol. 46 (2012) 7166-7173. doi:10.1021/es300645m.

[268] M. Engel, B. Chefetz, Adsorption and desorption of dissolved organic matter by carbon nanotubes: Effects of solution chemistry, Environ. Pollut. 213 (2016) 9098. doi:https://doi.org/10.1016/j.envpol.2016.02.009.

[269] H. Hyung, J.-H. Kim, Natural Organic Matter (NOM) Adsorption to Multi-Walled Carbon Nanotubes: Effect of NOM Characteristics and Water Quality Parameters, Environ. Sci. Technol. 42 (2008) 4416-4421. doi:10.1021/es702916h.

[270] D. Harikishore Kumar Reddy, K. Vijayaraghavan, J.A. Kim, Y.-S. Yun, Valorisation of post-sorption materials: Opportunities, strategies, and challenges, Adv. Colloid Interface Sci. 242 (2017) 35-58. doi:https://doi.org/10.1016/j.cis.2016.12.002.

[271] G. San Miguel, S.D. Lambert, N.J.D. Graham, The regeneration of field-spent granular-activated carbons, Water Res. 35 (2001) 2740-2748. doi:https://doi.org/10.1016/S0043-1354(00)00549-2.

[272] Y. Feng, D.D. Dionysiou, Y. Wu, H. Zhou, L. Xue, S. He, L. Yang, Adsorption of dyestuff from aqueous solutions through oxalic acid-modified swede rape straw: Adsorption process and disposal methodology of depleted bioadsorbents, Bioresour. Technol. 138 (2013) 191-197. doi:https://doi.org/10.1016/j.biortech.2013.03.146. 


\section{VITA}

On January $6^{\text {th }}, 1984$, Mohamed Bayati was born in Kirkuk, Iraq. He received his Bachelor's and Master's degrees from Tikrit University with a major in Civil Engineering and Environmental Engineering on July 2005 and July 2010 respectively.

He has over 14 years research experience related to environmental engineering including wastewater treatment, membrane separation, membrane fabrication and modification, oil spill treatment, metabolomic profiling, fate and transport of nanomaterials in the environment, nanostructured materials, colloidal and interfacial processes, and environmental systems analysis. 